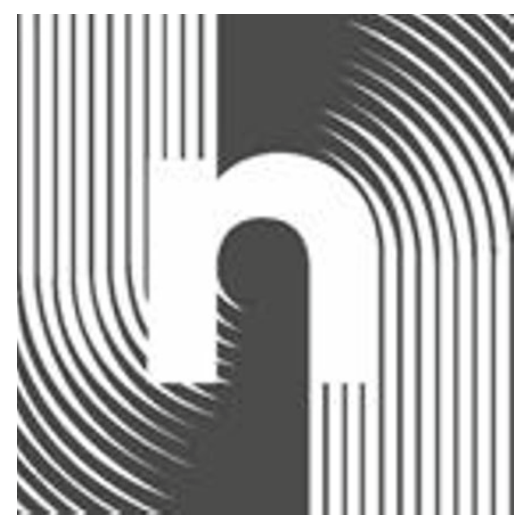

NEUROCRITICAL CARE SOCIETY $14^{\text {TH }}$ ANNUAL MEETING

\title{
ABSTRACT SUPPLEMENT
}

September 15-18, 2016

Gaylord National Resort \& Convention Center $\bullet$ National Harbor, MD 


\section{Table of Content}

\section{Oral Platform Presentations}

$4-21$

Sessions I and III platform presentations will take place in Prince George Exhibit Hall A. Session II will be in the main symposium room.

$\begin{array}{lll}\text { Session I } & \text { Friday, September 16 } & \text { 4:30pm - 5:30 pm } \\ \text { Session II } & \text { Saturday, September 17 } & 11: 00 \mathrm{am}-12: 00 \mathrm{pm} \\ \text { Session III } & \text { Saturday, September } 17 & 4: 30 \mathrm{pm}-5: 30 \mathrm{pm}\end{array}$

Poster Sessions

$22-302$

Posters can be viewed in Prince George Exhibit Hall A on Friday and Saturday from 5:30pm6:30pm.

\section{Basic Science}

Head and Spine Trauma 24

ICU Organization and Technology. $25-27$

Ischemic Stroke. $28-29$

Other.... $30-32$

Seizures 33

Subarachnoid hemorrhage. 34

Clinical $35-256$

Disorders of Consciousness. $45-51$

Head and Spine Trauma. $52-78$

ICU Organization and Technology $79-92$

Intracerebral hemorrhage. $93-118$

Ischemic Stroke. $119-134$

Medical Issues in the NeurolCU. $135-154$

Muscle and Nerve Disorders $155-156$

Other.... $157-187$

Pediatric NeurolCU $188-193$

Peri-Operative Management. $194-197$

Seizures $198-213$

Subarachnoid hemorrhage. $214-249$

Temperature Modulation $250-256$ 
Case Reports.

257-302

Disorders of Consciousness

$260-261$

Head and Spine Trauma.

$262-264$

ICU Organization and Technology.

$265-266$

Intracerebral hemorrhage....

$267-270$

Ischemic Stroke

$271-273$

Medical Issues in the NeurolCU

$274-278$

Muscle and Nerve Disorders 279

Other $280-289$

Peri-Operative Management. $290-292$

Seizures. $293-298$

Subarachnoid hemorrhage.... $299-301$

Temperature Modulation. 302 


\section{ORAL PLATFORM PRESENTATIONS I \\ Friday, September 16• 4:30 pm - 5:30 pm \\ Prince George Exhibit Hall A}

\section{Santosh Murthy}

Factors Associated with Permanent Cerebrospinal Fluid Shunting In Patients with Spontaneous Intraventricular Hemorrhage- Results from the Clot Lysis: Evaluating

\section{Kyle Hobbs}

MRI Characteristics of Early Seizures after Intracerebral Hemorrhage

\section{Stuart Fraser}

Race is a Predictor of Withdrawal of Life Support from Patients with Intracerebral Hemorrhage

\section{Wesley Baker}

Noninvasive Monitoring of Critical Closing Pressure with Near-infrared Light

\section{Lynze Franko}

Subdural Hematoma Patient Characteristics Associated with Unexpected 30-Day Readmission 


\section{ORAL PLATFORM PRESENTATIONS II}

Saturday, September 17 11:00 am - 12:00 pm

Main Symposia Hall (Translational Science Session)

Ivan da Silva- Best Abstract Award

Zika Virus Associated Neurological Complications in Adults

Vahid Eslami- Young Investigator Award

Influence of Intracerebral Hemorrhage Location in Patients with Spontaneous Intraventricular Hemorrhage- Results from the Clot Lysis: Evaluating Accelerated Resolution of Intraventricular Hemorrhage (CLEAR) III trial

\section{Stephen Trevick}

Decision Making and Palliative Care in the Neurosciences Intensive Care Unit: Family

Experiences and Outcomes

\section{Michael Reznik}

Long-Term Risk of Seizures in Survivors of Sepsis

\section{Ava Puccio}

Comparison of Progesterone Cerebrospinal and Serum Levels in Severe TBI Enrolled in SyNAPSE Trial 


\section{ORAL PLATFORM PRESENTATIONS III \\ Saturday, September $17 \bullet$ 4:30 pm - 5:30 pm \\ Prince George Exhibit Hall A}

\section{Thomas Quinn}

"The wrong decision is the one you can't live with." - A mixed-methods study of surrogate decision-making in the Neuro-ICU

\section{Stefan Wolf}

Does Early Lumbar Cerebrospinal Fluid Drainage Lead To A Better Outcome In Aneurysmal Subarachnoid Hemorrhage?

\section{Daniel Hänggi}

Safety, Tolerability, Pharmacokinetics and Efficacy of Intraventricular Sustained Release

Nimodipine (EG-1962) For Subarachnoid Hemorrhage

\section{Pitchaiah Mandava}

Modeling Outcome after Intracerebral Hemorrhage Indicates No Benefit and Potential Harm from Aggressive Blood Pressure Management

TBA - Late Breaking

TBA - Late Breaking 
OralPlatform Presentation

Friday, September 16• 4:30 pm - 5:30 pm

Prince George Exhibit Hall A

\title{
Factors Associated with Permanent Cerebrospinal Fluid Shunting In Patients With Spontaneous Intraventricular Hemorrhage- Results from the Clot Lysis: Evaluating Accelerated Resolution of Intraventricular Hemorrhage (CLEAR) III trial
}

\author{
Santosh Murthy', Jharna J. Shah ${ }^{2}$, Rachel R. Dlugash ${ }^{2}$, Nichol N. McBee ${ }^{2}$, Francois F. Aldrich ${ }^{3}$, Jean- \\ Louis J. Caron ${ }^{4}$, Daniel D. Hanley' ${ }^{2}$,Wendy W. Ziai . \\ ${ }^{1}$ Weill Cornell Medicine, New York, USA, ${ }^{2}$ Johns Hopkins University, Baltimore, USA, ${ }^{3}$ University of \\ Maryland, Baltimore, USA, ${ }^{4}$ University of Texas, San Antonio, USA.
}

\section{Introduction:}

CLEAR III, a randomized, double-blinded, placebo-controlled trial was conducted to determine if pragmatically employed extraventricular drainage (EVD) plus intraventricular alteplase improved outcome by removing IVH and controlling intracranial pressure (ICP) in comparison to EVD plus saline. Given the paucity of data on permanent cerebrospinal fluid (CSF) shunts in primary IVH, we studied predictors of shunting in the CLEAR population.

\section{Methods:}

CLEAR III randomized 500 subjects to receive up to 12 doses of alteplase or $0.9 \%$ saline $\mathrm{Q} 8 \mathrm{H}$ via the EVD until 3rd \& 4th ventricles opened. Our predictor variable was permanent CSF shunt. Outcome measures were predictors of shunting, and blinded assessment of modified Rankin scale (mRS) at 180 days.

\section{Results:}

CSF shunting was performed in 90 patients $(18 \%)$ at a median of 18 days (Interquartile Range, IQR:1330) from IVH onset and was not different between treatment groups. Patient demographics and IVH characteristics were similar amongst patients with and without shunts. In the multivariate analysis, African American race (Odds Ratio[OR]:1.80, 95\% Confidence Interval $\{\mathrm{Cl}\}: 1.08-3.08 ; \mathrm{p}=0.024)$, total doses of study agent (OR:1.07, Cl:1.01-1.14; $p=0.041$ ), daily drainage CSF per10cc (OR:1.08, Cl:1.05- 1.12; p30 $\mathrm{mmHg}(\mathrm{OR} 1.71, \mathrm{Cl}: 1.02-2.85 ; \mathrm{p}=0.043)$ were significantly associated with increased odds of CSF shunting, while end-of-treatment IVH volume, maximum CSF protein, thalamic obstruction were not. Patients who had CSF shunts had lower 180-day mortality but similar functional outcome, compared to patients without shunts.

\section{Conclusions:}

Among patients with primary IVH requiring emergency CSF diversion, those with persistently elevated ICP and high CSF output are at increased odds of developing persistent hydrocephalus, necessitating permanent ventricular shunting. The association with African American race may be more complex. These factors may assist in predicting need for permanent CSF diversion and could improve management of this condition. 


\title{
Oral Platform Presentation \\ Friday, September 16• 4:30 pm - 5:30 pm \\ Prince George Exhibit Hall A
}

\section{MRI Characteristics of Early Seizures After Intracerebral Hemorrhage}

\author{
Kyle S. Hobbs, Michael M. Mlynash, Soren S. Christensen, Anna A. Finley-Caulfield, Marion M. \\ Buckwalter, Chitra C. Venkatasubramanian.
}

Stanford University / Department of Neurology and Neurological Sciences, Stanford, CA, USA.

\section{Introduction:}

We hypothesized that patients who have perihematomal ischemia $(\mathrm{PHI})$ are more likely to have early post-ICH seizures (i.e. $<30$ days).

\section{Methods:}

277 patients from the prospective NIH funded DASH1 study ( $>18$ years, spontaneous $\mathrm{ICH} / \mathrm{IVH}$ ) were included. All patients had multimodal MRI. Imaging variables included ICH volume, location and number, etiology, early/delayed IVH, hydrocephalus, midline shift, SAH, microbleeds (lobar/deep), leukoaraiosis, and visible ischemia on ADC scored as perihematomal or remote. EEG monitoring was performed for clinical indications. Comparisons between those who did and did not have seizures (clinical and/or electrographic) was done with t-test or Mann-Whitney test for continuous variables and $\mathrm{x} 2$ or Fisher's Exact test for categorical data.

\section{Results:}

Eighty-six patients had EEG; thirty-two (12\%) had early seizures (29 clinical, 3 electrographic only) at a median of day 2. Patients with early seizures compared to those without were more likely to have lobar ( $78 \%$ vs. $41 \%)$ than deep ICH (19\% vs. $53 \%, p=0.001)$, or SAH ( $41 \%$ vs. $13 \% p<0.001)$. Volume of $\mathrm{ICH}$, leukoariosis, microbleeds and $\mathrm{PHI}(\mathrm{n}=74,31 \%$ vs. $30 \%)$ did not differ between groups. Prevalence of diabetes ( $6 \%$ vs. $24 \% P=0.02)$, hypertension $(57 \%$ vs. $72 \%, p=0.08)$ and remote ischemia $(n=52)$ was also lower in those with seizures $(7 \%$ vs $22 \%, p=0.07)$. Patients with seizures differed in etiology and were more likely in those with sinus thrombosis $(16 \%$ vs $2 \%, p=0.01)$ and less likely in those with hypertension ( $25 \%$ vs. $44 \%, p=0.01)$.

\section{Conclusions:}

Patients with early seizures after $\mathrm{ICH}$ had predominantly lobar $\mathrm{ICH}, \mathrm{SAH}$, and were less likely to exhibit classic cardiovascular risk factors. $\mathrm{PHI}$ assessed qualitatively on ADC was not predictive of seizures. Quantitative assessment of perihematomal diffusivity with addition of perfusion may be a better predictor for early seizures. 


\title{
Oral Platform Presentation \\ Friday, September 16• 4:30 pm - 5:30 pm \\ Prince George Exhibit Hall A
}

\section{Race is a Predictor of Withdrawal of Life Support from Patients with Intracerebral Hemorrhage}

\author{
Stuart M. Fraser ${ }^{1}$, Glenda G. Torres ${ }^{2}$, Chunyan C. Cai ${ }^{3}$, Alex A. Choi ${ }^{1}$,Daniel D. Saenz ${ }^{2}$, Anjail A. \\ Sharrief ${ }^{1}$, Nancy N. Edwards ${ }^{1}$, Kiwon K. Lee ${ }^{1}$, James J. Grotta ${ }^{4}$, Tiffany T. Chang ${ }^{1}$.
}

\begin{abstract}
${ }^{1}$ Department of Neurology, University of Texas Health Science Center at Houston, Houston, TX, USA, ${ }^{2}$ Department of Neurosurgery, University of Texas Health Science Center at Houston, Houston, TX, USA,

${ }^{3}$ Department of Internal Medicine, University of Texas Health Science Center at Houston, Houston, TX, USA, ${ }^{4}$ Memorial Hermann Hospital, Houston, TX, USA.
\end{abstract}

\begin{abstract}
Introduction:
Medical and socioeconomic factors may impact decisions to change the goals of care for patients with intracerebral hemorrhage $(\mathrm{ICH})$ to comfort measures only $(\mathrm{CMO})$.
\end{abstract}

\section{Methods:}

We reviewed prospectively collected data on patients with ICH between 2009 and 2015. Data included baseline patient demographics, medical comorbidities, admission Glasgow Coma Scale (GCS), National Institute of Health Stroke Scale (NIHSS), ICH score, and intraventricular hemorrhage (IVH) volume. We conducted multivariable logistic regression analysis to identify predictors of change to CMO status.

\section{Results:}

Of 200 patients included in the analysis, $39(19.5 \%)$ were made CMO during the hospitalization. Age, gender, marital status, religion, insurance status, age-adjusted Charlson comorbidity index, and tobacco, ethanol, and cocaine use were similar between the two groups. Race was significantly different between the CMO (black $15.4 \%$, white $51.3 \%$, other $33.3 \%$ ) and non-CMO groups (black $39.8 \%$, white $46 \%$, other $14.3 \% ; p=0.0027)$. Patients changed to $\mathrm{CMO}$ had higher mean income based on zip code $(\$ 59,534$ vs. $\$ 50,128 ; p=0.0209$ ), higher median NIHSS (23 vs. 16 ; $p<0.0001)$, higher ICH score (2.7 vs. 1.5; $p<0.0001)$, lower median GCS (7 vs. $13 ; p<0.001)$, and larger median IVH volume $(16.4 \mathrm{~mL}$ vs. $1.4 \mathrm{~mL} ; p$ $<0.0001)$ compared with non-CMO patients. Following multivariable analysis, factors associated with CMO were GCS (OR 0.75, 95\% Cl 0.66-0.85, p<0.0001), IVH volume (OR 1.04 95\%, Cl 1.01-1.06, $\mathrm{p}=0.0016$ ), black race (OR $0.24,95 \% \mathrm{Cl} 0.07-0.81, \mathrm{p}=0.0217$ ), transfer from an outside hospital (OR $2.46,95 \% \mathrm{Cl} 0.99-6.15, \mathrm{p}=0.0537$ ), and unemployed work status (OR 2.87, 95\% Cl 1.01-8.20, $\mathrm{p}=0.0487$ ).

\section{Conclusions:}

Lower GCS score, higher IVH volume, employment status, and race were independent predictors of CMO. Black patients were $75 \%$ less likely to withdraw life support than other races. Providers should be aware of potential racial differences in goals of care and acceptance of palliative care. Further study is warranted to understand potential underlying factors. 


\title{
Oral Platform Presentation \\ Friday, September 16• 4:30 pm - 5:30 pm \\ Prince George Exhibit Hall A
}

\section{Noninvasive Monitoring of Critical Closing Pressure with Near-infrared Light}

\author{
Wesley B. Baker ${ }^{1}$, Ashwin A. Parthasarathy ${ }^{2}$, Kimberly K. Gannon ${ }^{3}$, Venki V. Kavuri ${ }^{2}$, Michael M. Mullen ${ }^{3}$, \\ Arjun A. Yodh ${ }^{2}$, Ramani R. Balu', W. Andrew W. Kofke ${ }^{3}$. \\ ${ }^{1}$ University of Pennsylvania / Anesthesiology and Critical Care, Philadelphia, PA, USA, ${ }^{2}$ University of \\ Pennsylvania / Physics and Astronomy, Philadelphia, PA, USA, ${ }^{3}$ University of Pennsylvania / Neurology, \\ Philadelphia, PA, USA.
}

\section{Introduction:}

The critical closing pressure (CrCP) is the arterial blood pressure (abp) at which cerebral blood flow (CBF) approaches zero. CrCP depends on intracranial pressure, and abp-CrCP is a promising noninvasive proxy for cerebral perfusion pressure. We developed a novel technique to measure $\mathrm{CrCP}$ with near-infrared light, and tested it in 14 healthy adults and two TBI patients.

\section{Methods:}

Diffuse correlation spectroscopy (DCS) employs near-infrared light to measure local, microvascular cerebral blood flow continuously at the bedside. DCS is noninvasive with deep tissue penetration $(\sim 1 \mathrm{~cm})$ and excellent time resolution $(20-100 \mathrm{~Hz})$. We utilized DCS to measure pulsatile blood flow oscillations in cerebral arterioles in the prefrontal cortex for 5 minutes in 14 healthy adults. Simultaneously, middle cerebral artery flow velocity and abp in the finger were monitored noninvasively with transcranial Doppler ultrasound (TCD) and a Finapres, respectively. For each subject, we measured $\mathrm{CrCP}$ using two techniques, i.e., CrCPtcd and CrCPdcs. CrCPtcd was obtained with Michel's method based on abp and flow velocity assessed with TCD. CrCPdcs was calculated using DCS measurements of arteriole blood flow in place of the TCD data. We further measured CrCPdcs in two TBI patients.

\section{Results:}

We found good agreement between the TCD and DCS measures of critical closing pressure $(R=0.8$, slope $=0.93 \pm 0.09$, mean difference $=0.6 \pm 4.8 \mathrm{mmHg}$ ). The averages across healthy subjects are $\mathrm{CrCPdcs}=13.6 \pm 7.5 \mathrm{mmHg}$ and $\mathrm{CrCPtcd}=13.0 \pm 7.8 \mathrm{mmHg}$ (mean $\pm \mathrm{SD}$ ). The CrCPdcs measurements in the two TBI subjects with normal ICP were 13.3 and $15.5 \mathrm{mmHg}$.

\section{Conclusions:}

DCS is a novel technique for noninvasive, continuous monitoring of $\mathrm{CrCP}$ at the bedside. We validated it against TCD, and demonstrated its use in the neurocritical care environment. DCS probes are well- suited for monitoring over prolonged periods of time and can be secured anywhere on the head. 


\title{
Oral Platform Presentation
}

Friday, September 16• 4:30 pm - 5:30 pm

Prince George Exhibit Hall A

\section{Subdural Hematoma Patient Characteristics Associated with Unexpected 30-Day Readmission}

\author{
Lynze Franko ${ }^{1}$, Kyle K. Sheehan ${ }^{2}$, Venkatakrishna V. Rajajee ${ }^{3}$, Craig C. Williamson ${ }^{3}$.
}

\author{
${ }^{1}$ University of Michigan School of Medicine, Ann Arbor, MI, USA, ${ }^{2}$ University of Michigan Department of \\ Neurosurgery and Neurology, Ann Arbor, MI, USA, ${ }^{3}$ University of Michigan Departments of Neurosurgery \\ and Neurology, Ann Arbor, MI, USA.
}

\section{Introduction:}

Thirty-day readmission rates are proposed as a hospital quality measure, with Medicare imposing financial penalties on hospitals with high readmission rates for specific conditions. The incidence and clinical predictors of 30-day readmission rates in subdural hematoma (SDH) patients have not been described.

\section{Methods:}

A retrospective review of patients with SDH admitted to the University of Michigan neurological ICU 7/2011-7/2015 was performed. Data were abstracted from the electronic medical record by members of the study team using standardized templates and definitions. Readmitted and non-readmitted patients were compared, and a multivariable logistic regression analysis of factors associated with readmission was performed.

\section{Results:}

Of 180 total patients, 16 died during hospitalization and were not included in analysis. Not counting two patients with planned readmissions, $45(27.4 \%)$ patients were unexpectedly readmitted within 30 days. Factors associated with unexpected readmission include non-operative management $(73.3 \%$ vs. $52.3 \%$, $p=0.02)$, performance of burrhole drainage without subdural drain placement $(38.5 \%$ of surgeries vs. $7.1 \%, p=0.008)$ and female gender $(46.7 \%$ vs. $31.1 \%, p=0.06)$. Age, Charlson comorbidity index, dementia, traumatic etiology, hematoma characteristics (hyperdense, iso/hypodense and mixed), midline shift, GCS and focal neurological deficits were not associated with unexpected readmission. In a multivariable logistic regression model including the potential confounders of age, gender, midline shift, GCS and presence of focal neurological deficits, the association with conservative management remained significant $(\mathrm{OR}=2.96,95 \% \mathrm{Cl} 1.07-8.2, \mathrm{p}=0.04)$. Twenty-two $(48.9 \%)$ readmissions were directly attributable to the SDH, with patients most often re-presenting with headache as their primary complaint. Fourteen (31.1\%) patients underwent SDH evacuation during their readmission.

\section{Conclusions:}

The 30-day unexpected readmission rate for subdural hematoma patients at a single tertiary care neurological ICU was substantial. Non-operative management was significantly associated with readmission, even accounting for likely confounders. 


\section{Oral Platform Presentation -Best Abstract 2016 \\ Saturday, September 17 11:00 am - 12:00 pm \\ Main Symposia Hall (Translational Science Session)}

\section{Zika Virus Associated Neurological Complications in Adults}

Ivan R. da Silva.

Department of Neurology, Fiocruz, Rio de Janeiro, Brazil.

\section{Introduction:}

Zika virus (ZIKV) is a rapidly evolving epidemic that may be associated with severe neurological sequelae in adults, beyond its effects on the developing fetus.

\section{Methods:}

A prospective study was conducted in Rio de Janeiro, Brazil from 12/10/2015-5/10/2016 enrolling patients with neurological complications preceded by symptoms suggestive of ZIKV infection (rash, conjunctivitis, fever). Clinical data, imaging, EMG/NCS studies, and blood and CSF molecular (RT-PCR) and serological studies for ZIKV, Dengue and Chikungunya viruses were evaluated. Guillain-Barre Syndrome (GBS) was diagnosed following Brighton criteria.

\section{Results:}

34 patients were enrolled with a median age of 42 (range 22-67): 20 (59\%) with GBS (2 with AMAN and 2 AMSAN), $2(6 \%)$ with CIDP, $6(18 \%)$ with ADEM, $3(8 \%)$ with transverse myelitis, $2(6 \%)$ with encephalitis and $1(3 \%)$ with rapidly progressive dementia. The rate of GBS hospital admission increased from a preZIKV baseline of 0.67 cases/month to 4 cases/month. The median time to onset of neurological symptoms after rash was $3 \mathrm{~d}$ for encephalitis and 7-10d for all other syndromes. RT-PCR for ZIKV was positive in 2 GBS and 2 encephalitis patients, while 30 patients were ZIKV IgM seropositive (6 were also CSF ZIKV IgM positive). All patients were Dengue IgG/lgM negative except one patient (Dengue IgG seropositive, ZIKV IgM positive in blood and CSF). 12 (35\%) required ICU admission. One patient with encephalitis died and one patient with rapidly progressive dementia remains in a vegetative state. All 22 GBS/CIDP patients were ambulatory at hospital discharge following IVIG administration, and 3 patients with transverse myelitis clinically improved with steroids or IVIG.

\section{Conclusions:}

ZIKV may be associated with a large spectrum of life-threatening neurocritical complications in adults. Understanding the population at risk for these neurological complications and the long term impact on outcomes will inform efforts directed at vector control, and patient management. 


\title{
Oral Platform Presentation - Young Investigator Award 2016 \\ Saturday, September 17 11:00 am - 12:00 pm \\ Main Symposia Hall (Translational Science Session)
}

\begin{abstract}
Influence of Intracerebral Hemorrhage Location in Patients With Spontaneous Intraventricular Hemorrhage- Results from the Clot Lysis: Evaluating Accelerated Resolution of Intraventricular Hemorrhage (CLEAR) III trial
\end{abstract}

Vahid Eslami ${ }^{1}$, Saman S. Nekoovaght-Tak ${ }^{2}$, Pouya P. Tahsili Fahadan ${ }^{2}$, Rachel R. Dlugash ${ }^{2}$, Nichol N. McBee ${ }^{2}$, Karen K. Lane ${ }^{2}$, Penelope P. Keyl ${ }^{3}$, Isaam I. Awad ${ }^{4}$, Daniel D. Hanley', Wendy W. Ziai'.

${ }^{1}$ Department of Neurology, Johns Hopkins University School of Medicine., Baltimore, MD, USA, ${ }^{2}$ Department of Neurology, Johns Hopkins University School of Medicine, baltimore, MD, USA, ${ }^{3}$ Sr. Biostatistician, Johns Hopkins University School of Medicine, Baltimore, MD, USA, ${ }^{4}$ Section of Neurosurgery, University of Chicago Pritzker School of Medicine, Baltimore, MD, USA.

\section{Introduction:}

The location of intracerebral hemorrhage $(\mathrm{ICH})$ is typically identified by one primarily affected anatomic region. $\mathrm{ICH}$ may extend into multiple brain areas, distend tissues, and break through the ventricular wall. We provide an anatomic framework for estimating $\mathrm{ICH}$ engagement and its relevance to stroke outcomes.

\section{Methods:}

We analyzed diagnostic computed tomography (CT) scans from all 500 patients from the Clot Lysis: Evaluating Accelerated Resolution of Intraventricular Hemorrhage (CLEAR) III trial. We measured ICH engagement with specific anatomic regions, and estimated association of each region with blinded assessment of stroke severity measures at day 30 and 180 .

\section{Results:}

On 500 diagnostic CT scans, the frequency of involvement of each anatomic region was: 332 (65.6\%) thalamus, $219(43.8 \%)$ caudate, $188(37.6 \%)$ posterior (PLIC) and $108(21.6 \%)$ anterior (ALIC) limb of internal capsule, $110(22.0 \%)$ putamen, $123(24.6 \%)$ globus pallidus, and $29(5.8 \%)$ lobar. Median (interquartile range) $\mathrm{ICH}$ volume was $8.5(4.2,14.6) \mathrm{ml}$. Other presenting factors were intraventricular hemorrhage (IVH) volume (28ml), Glasgow coma scale (GCS) (8), and NIHSS (21). On multivariable analysis adjusting for ICH and IVH volume, age, GCS and study treatment (saline vs. alteplase), thalamic location was significantly associated with all poor outcome measures (day 30/180 mortality, modified Rankin scale 4-6, Barthel Index (BI) $\leq 85$, NIHSS $>4$, mobility score (stroke impact scale) and day 7 GCS $\leq 8$. Lesions of the PLIC were associated with poor Day $180 \mathrm{BI}$ and mobility score while caudate, ALIC and lobar lesions were associated with good outcomes.

\section{Conclusions:}

Clinician determined anatomic regions of $\mathrm{ICH}$ engagement are independent predictors of day 30 and 180 functional outcome scores in patients with small ICH volumes ( $<30 \mathrm{cc})$ and large IVH. Qualitative clinical inspection of CT images demonstrates thalamic and PLIC lesions are the most important location determinants of mortality and residual disability in this patient population. 


\section{Oral Platform Presentation}

Saturday, September 17 • 11:00 am - 12:00 pm

Main Symposia Hall (Translational Science Session)

\section{Decision Making and Palliative Care in the Neurosciences Intensive Care Unit: Family Experiences and Outcomes}

Stephen A. Trevick, Aaron A. Lord.

NYU School of Medicine/Department of Neurology, New York City, NY, USA.

${ }^{* * * *}$ Permission was not granted to print this abstract ${ }^{* * * *}$ 


\title{
Oral Platform Presentation \\ Saturday, September 17 • 11:00 am - 12:00 pm Main Symposia Hall (Translational Science Session)
}

\section{Long-Term Risk of Seizures in Survivors of Sepsis}

\author{
Michael E. Reznik ${ }^{1}$, Alexander A. Merkler ${ }^{1}$, Ali A. Mahta ${ }^{1}$, Santosh S. Murthy ${ }^{2}$, Jan J. Claassen ${ }^{3}$, Hooman \\ H. Kamel ${ }^{2}$.
}

\footnotetext{
${ }^{1}$ Dept. of Neurology, Weill Cornell Medicine; Dept. of Neurology, Columbia University, New York, NY, USA, ${ }^{2}$ Department of Neurology, Weill Cornell Medicine, New York, NY, USA, ${ }^{3}$ Department of Neurology, Columbia University Medical Center, New York, NY, USA.
}

\section{Introduction:}

Sepsis has been associated with concurrent neurological dysfunction including seizures; however, it is unknown whether survivors of sepsis also face an increased long-term risk of seizures.

\section{Methods:}

We performed a population-based cohort study on all adult patients in California from 2005-2011, New York from 2006-2013, and Florida from 2005-2013 using administrative claims data from the Healthcare Cost and Utilization Project (HCUP). Using validated algorithms, we identified all adult patients who were hospitalized with sepsis. Our outcome of interest was any subsequent emergency department visit or hospitalization for seizure. As we were interested in new-onset seizures, we excluded patients with seizures prior to or during the index hospitalization for sepsis. Poisson regression was used to calculate age-, sex-, and race-standardized incidence rate ratios (IRR) based on publicly available demographic data. To confirm our findings, we applied these methods to claims data from a nationally-representative $5 \%$ sample of Medicare beneficiaries.

\section{Results:}

We identified 842,723 patients with sepsis, in whom the cumulative rate of seizures was $6.7 \%(95 \% \mathrm{Cl}$ 6.6-6.8\%) over 8 years of follow-up. As compared to demographic controls, the IRR for new-onset seizure was $5.0(95 \% \mathrm{Cl} 4.9-5.0)$ in all patients with sepsis, and $7.5(95 \% \mathrm{Cl} 7.3-7.7)$ in those with concurrent neurological dysfunction. The analysis using Medicare data confirmed that sepsis was associated with an increased long-term risk of seizures, with an IRR of $2.7(95 \% \mathrm{Cl} 2.6-2.8)$. This prompted a post-hoc analysis using the HCUP dataset, which showed a similar IRR of $2.8(95 \% \mathrm{CI} 2.8-2.9)$ in patients $>65$ years of age, but $10.3(95 \% \mathrm{Cl} 10.2-10.5)$ in patients $<65$ years old.

\section{Conclusions:}

Survivors of sepsis face a significant long-term risk of developing seizures. Our results suggest that sepsis should be considered a type of acute brain injury, which may lead to permanent neurological sequelae. 


\title{
Oral Platform Presentation \\ Saturday, September 17 • 11:00 am - 12:00 pm \\ Main Symposia Hall (Translational Science Session)
}

\section{Comparison of Progesterone Cerebrospinal and Serum Levels in Severe TBI Enrolled in SyNAPSE Trial}

\author{
Ava M. Puccio ${ }^{1}$, Samuel S. Poloyak ${ }^{2}$, Margaret M. Minnigh ${ }^{2}$, Jordan J. Brooks ${ }^{1}$, David D. Okonkwo ${ }^{1}$. \\ ${ }^{1}$ University of Pittsburgh/Neurosurgery, Pittsburgh, PA, USA, ${ }^{2}$ University of Pittsburgh/Pharmacy, \\ Pittsburgh, PA, USA.
}

\section{Introduction:}

Experimental models of TBI have shown positive effects of progesterone administration on outcome, with attenuation of cerebral edema, inflammation and apoptosis. Despite positive translational, phase II trials in humans being favorable, two recent phase III trials (SyNAPSE and ProTECT) were stopped for futility, with no difference in 6-month neurological outcome. It has been theorized that the optimal dose of treatment was too high, or a more sensitive outcome measure needs to be utilized. The purpose of this study was to examine the serum and cerebrospinal fluid (CSF) levels of progesterone of randomized participants who were enrolled in the BHR Pharma sponsored Phase III trial, Study of the Neuroprotective Activity of Progesterone in Severe Traumatic Brain Injuries (SyNAPSE).

\section{Methods:}

The participants (randomized to progesterone treated or placebo control) had serum and CSF collected under a site, IRB approved protocol. Samples were prospectively collected pre-treatment and up to 5 days post-treatment, processed and stored at $-80 \mathrm{oC}$ prior to batch processing for mass spectroscopy.

\section{Results:}

14 patients (mean age $36.3 \pm 15.7$, median GCS score 6 ) samples sets (serum and CSF) were analyzed for progesterone levels utilizing mass spectroscopy. The mean progesterone levels following experimental dosing for the placebo control group were 24 , and $1,102 \mathrm{pg} / \mathrm{ml}$, and the randomized progesterone group were 1,282 , and 273,708 ; serum and CSF respectively.

\section{Conclusions:}

In this exploratory analysis, CSF levels in the progesterone treated group were variable and not as supratheraputic compared to serum levels. This study highlights an additional factor that needs to be considered in the designing of clinical trials in TBI. Not only does the heterogeneity of the injury and subsequent outcome measures need to be refined, but the biomarker of pK levels also needs to be analyzed in CSF as well as serum to determine if the treatment is reaching the target organ, the brain. 


\title{
Oral Platform Presentation \\ Saturday, September 17 • 4:30 pm - 5:30 pm \\ Prince George Exhibit Hall A
}

\begin{abstract}
"The wrong decision is the one you can't live with." - A mixed-methods study of surrogate decision-making in the Neuro-ICU
\end{abstract}

\author{
Thomas Quinn", Jesse J. Moskowitz', Lori L. Shutter ${ }^{2}$, Rob R. Goldberg ${ }^{3}$, Nananda N. Col' ${ }^{4}$, Kathy K. \\ Mazor $^{5}$, Susanne S. Muehlschlegel.
}

\begin{abstract}
${ }^{1}$ University of Massachusetts Medical School, Worcester, MA, USA, ${ }^{2}$ University of Pittsburgh Medical Center / Dept. of Neurology and Critical Care, Pittsburgh, PA, USA, ${ }^{3}$ University of Massachusetts Medical School / Dept. of Quantitative Health Sciences, Worcester, MA, USA, ${ }^{4}$ University of New England / Dept. of Internal Medicine, Biddeford, ME, USA, ${ }^{5}$ University of Massachusetts Medical School / Dept. of Primary Care, Worcester, MA, USA, ${ }^{6}$ University of Massachusetts Medical School / Dept. of Neurology, Worcester, MA, USA.
\end{abstract}

\section{Introduction:}

The creation of a meaningful shared decision-making tool for goals-of-care (GOC) decisions in NeuroICU patients requires the understanding of surrogate decision makers' perceptions of current physician prognostication and communication practices and how they might influence GOC decision-making.

\section{Methods:}

We conducted a mixed-methods study with semi-structured interviews in 16 surrogate decision-makers of critically-ill traumatic brain injury patients from two level-1 trauma-centers in the Northeast. Interviews explored surrogates' perception of communication by physicians during GOC discussions. We surveyed examples of good and poor communication, reasons for surrogates' sentiments, and explored the most helpful approach by physicians in communicating prognosis. Transcribed interviews were analyzed using NVIVO-software with the investigator-triangulated-inductive-framework-approach. Theme saturation determined the final sample size.

\section{Results:}

Overall, $90 \%$ of respondents provided examples of helpful physician communication processes and content: time and opportunity to ask questions during ICU-rounds and family meetings was consistently identified as most important, providing "some kind of control, being more equipped or confident to make decisions". Over half reported that poor communication, perceived lack of continuity and physician-tophysician communication breakdown was a major decision-making barrier. $60 \%$ reported reliance on non -physician staff for clarification of medical information during the decision-making process. Prognostic uncertainty was described as "the scariest thing of all", and a "constant source of doubt and fear"; only a minority of surrogates understood uncertainty as an expected part of the GOC discussion. While addressing prognosis, families recognized directness and candor as most helpful: they "could go elsewhere for hope; what [they] needed were the facts."

\section{Conclusions:}

As fundamental groundwork for a meaningful GOC shared decision-making tool in ciTBI (and other diseases), we identified important factors that impact surrogates' decision-making for GOC discussions. 


\title{
Oral Platform Presentation \\ Saturday, September 17 • 4:30 pm - 5:30 pm \\ Prince George Exhibit Hall A
}

\section{Does Early Lumbar Cerebrospinal Fluid Drainage Lead To A Better Outcome In Aneurysmal Subarachnoid Hemorrhage?}

\author{
Stefan Wolf ${ }^{1}$, The T. Earlydrain Study Group ${ }^{2}$. \\ ${ }^{1}$ Department of Neurosurgery, Charite Campus Virchow, Charite University Medicine, Berlin, Germany, \\ ${ }^{2}$ multiple, multiple, Germany.
}

\section{Introduction:}

In retrospective series, the use of a lumbar drain in patients with aneurysmal subarachnoid hemorrhage $(\mathrm{SAH})$ was repeatedly associated with a better neurologic outcome. Postulated mechanism of action is an improved clearance of the subarachnoid space and the basal cisterns from blood, leading to less vasospasm and infarctions. A prospective single-center study was able to establish the safety of an additional lumbar drain, but did not show an outcome benefit in good-grade SAH patients. Therefore, a large trial in SAH patients without severity restriction was warranted.

\section{Methods:}

Earlydrain was a prospective, randomized, multicenter trial in patients with aneurysmal SAH. Main inclusion criteria were aneurysm treatment within 48 hours after SAH and possibility to start lumbar CSF drainage within another 24 hours. Patients were randomized either to receive this lumbar drainage for 8 consecutive days with $5 \mathrm{ml} / \mathrm{h}$ or to standard treatment only. Outcome was assessed with the modified Rankin scale after 6 months.

\section{Results:}

300 patients in 17 centers in Germany, Switzerland and Canada entered the study and were randomized $1: 1$ to either receive lumbar drainage or conventional therapy. The last patient was recruited in January 2016, with final 6-months outcome expected to be available in July 2016. First results are to be presented at the 14th annual NCS meeting in National Harbor in September 2016.

\section{Conclusions:}

The results of the prospective multicenter Earlydrain study will provide evidence on the efficacy of a lumbar drain in aneurysmal subarachnoid hemorrhage. 


\title{
Oral Platform Presentation \\ Saturday, September 17 • 4:30 pm - 5:30 pm \\ Prince George Exhibit Hall A
}

\section{Safety, Tolerability, Pharmacokinetics and Efficacy of Intraventricular Sustained Released Nimodipine (EG-1962) for Subarachnoid Hemorrhage}

\author{
Daniel Hänggi ${ }^{1}$, R. Loch R. Macdonald ${ }^{2}$, Nima N. Etminan ${ }^{3}$, Hans Jakob H. Steiger ${ }^{4}$, Stephan S. Mayer ${ }^{5}$, \\ Francois F. Aldrich ${ }^{6}$, Michael M. Diringer ${ }^{7}$, Brian B. Hoh ${ }^{8}, \mathrm{~J} \mathrm{~J}$. Mocco $^{5}$, Poul P. Strange ${ }^{9}$, Herbert H. \\ Faleck $^{10}$, Michael M. Miller'.

\footnotetext{
${ }^{1}$ Department of Neurosurgery, Universitätsmedizin Mannheim, Universität Heidelberg, Mannheim, Germany, ${ }^{2}$ Department of Neurosurgery, St. Michael's Hospital, Toronto, Canada, ${ }^{3}$ University Hospital Mannheim, University of Heidelberg Mannheim, Dusseldorf, Germany, ${ }^{4}$ Heinrich-Heine-University, Dusseldorf, Germany, ${ }^{5}$ Mount Sinai Hospital, New York, USA, ${ }^{6}$ University of Maryland Medical Center, Baltimore, USA, ${ }^{7}$ Washington University, St. Louis, USA, ${ }^{8}$ University of Florida, Gainesville, USA, ${ }^{9}$
} \\ Integrated Medical Development, Princeton Junction, USA, ${ }^{10}$ Edge Therapeutics, Inc., Berkeley Heights,
} USA.

\section{Introduction:}

Treatments for aneurysmal subarachnoid hemorrhage (aSAH) remain inadequate. EG-1962 is a sustained release formulation of nimodipine for intraventricular delivery in order to avoid dose-limiting hypotension common with systemic administration. We conducted a phase 1/2a multicenter, controlled, randomized, open-label, dose escalation study to determine the maximum tolerated dose (MTD) and assess safety, tolerability, pharmacokinetics and efficacy at 90 days on the extended Glasgow outcome scale (GOSE) of a single intraventricular dose of EG-1962 (www.clinicaltrials.gov Identifier:

NCT01893190).

\section{Methods:}

Subjects with aSAH repaired by clipping or coiling were randomized within 60 hours of aSAH to EG-1962 or oral nimodipine if they were World Federation of Neurological Surgeons grade 2 to 4 and had a ventricular catheter. Cohorts of 12 subjects received 100, 200, 400, 600, 800 or 1200 mg EG-1962 (9 per cohort) or oral nimodipine (3 per cohort).

\section{Results:}

The MTD was 800 mg EG-1962. Plasma nimodipine concentrations with EG-1962 were sustained for 21 days and increased in a dose-dependent fashion. Cerebrospinal fluid nimodipine concentrations with EG 1962 were orders of magnitude higher than in plasma or with oral nimodipine. There was one serious adverse event related to EG-1962 and two EG-1962 dose limiting toxicities; all were without clinical sequelae. There was no EG-1962-related hypotension versus hypotension in $17 \%(3 / 18)$ with oral nimodipine. Favorable outcome (GOSE 6-8) was achieved in 27 of $45(60 \%)$ EG-1962 subjects (5/9 with $100,6 / 9$ with $200,7 / 9$ with $400,4 / 9$ with $600,5 / 9$ with $800 \mathrm{mg})$ and $5 / 18(28 \%)$ oral nimodipine subjects. Delayed cerebral ischemia (15/45 [33\%] EG-1962 versus 11/18 [61\%] oral nimodipine) and rescue therapy $(11 / 45[24 \%]$ versus $10 / 18$ [56\%]) were reduced in EG-1962 subjects. 


\section{Conclusions:}

Intraventricular EG-1962 was safe and tolerable to $800 \mathrm{mg}$, associated with sustained, dose-dependent nimodipine plasma concentrations, improved clinical outcome and reduced delayed cerebral ischemia and rescue therapy after aSAH. 


\title{
Oral Platform Presentation
}

Saturday, September 17 • 4:30 pm - 5:30 pm

Prince George Exhibit Hall A

\section{Modeling Outcome after Intracerebral Hemorrhage Indicates No Benefit and Potential Harm from Aggressive Blood Pressure Management}

\author{
Pitchaiah Mandava ${ }^{1}$, Santosh S. Murthy ${ }^{2}$, Yves Y. Samson ${ }^{3}$, Thomas T. Kent ${ }^{1}$. \\ ${ }^{1}$ Baylor College of Medicine and Michael E. DeBakey VA Medical Center, Houston, TX, USA, ${ }^{2}$ Baylor \\ College of Medicine, Houston, TX, USA, ${ }^{3}$ Urgences Cérébro-Vasculaires Hôpital Universitaire Pitié \\ Salpêtrière, Paris, France.
}

\section{Introduction:}

Outcome following Intra-Cerebral Hemorrhage $(\mathrm{ICH})$ is related to baseline factors such as severity of the initial neurological deficit, patient's age and hematoma volume. Unfortunately in all but the largest trials, imbalances in these baseline factors between arms are common, complicating analysis. To overcome this problem, we developed a method that pools the control arms of randomized clinical trials (RCTs) across a range of baseline factors, and compares an individual treatment arm against this pooled model at the studies own baseline without need for statistical correction. Here, we generated a model from the control arms of ICH RCTs. The model incorporates statistical variability to assess whether a treatment differs from expected outcome (Neurology 85:274-83, 2015). We tested the effect of blood pressure (BP) lowering interventions against the predicted outcome.

\section{Methods:}

Models for functional outcome (mRS 0-2) and mortality derived from $19 \mathrm{ICH}$ RCTs representing 3000 subjects were generated. Results from the 4 major published BP RCTs at the time of this writing were tested against the model at their baseline NIHSS, age and hematoma volume.

\section{Results:}

Good fit for both functional outcome (modified Rankin Scale, mRS0-2: R-Square:0.74; $p<0.001$ ), and mortality models were found (R-square:0.60; $\mathrm{p}<0.005)$. Control and treatment arm outcomes of ATACH, ICH-ADAPT, INTERACT and INTERACT-2 were plotted. No benefit was apparent as outcomes were mostly distributed along the control surface indicating no difference from predicted outcome. The tier 3 of ATACH, with a goal of SBP 110-140, demonstrated increased mortality and decreased mRS 0-2 than predicted from the pooled sample.

\section{Conclusions:}

Outcome models for mortality and mRS 0-2 were successfully developed. No benefit was seen from any BP intervention. The outcome models correctly predicted the negative outcome of ATACH-II. We suspect small differences reported as positive in prior trials were most likely due to random variation or baseline imbalance. 


\title{
Basic Science
}

\author{
All even numbered posters will present on Friday \\ All odd numbered posters will present on Saturday \\ All poster sessions are in Prince George Exhibit Hall A from 5:30pm-6:30pm
}

Head and Spine Trauma

\begin{tabular}{|c|c|c|l|}
\hline $\begin{array}{c}\text { Poster } \\
\text { Number }\end{array}$ & First Name & Last Name & Abstract Title \\
\hline & & $\begin{array}{l}\text { Cerebral blood flow thresholds for supply dependent glucose utilisation in } \\
\text { traumatic brain injury: A multi-tracer 15Oxygen and [18F]- } \\
\text { fluorodeoxyglucose positron emission tomography study }\end{array}$ \\
\hline
\end{tabular}

\section{ICU Organization and Technology}

\begin{tabular}{|c|l|l|l|}
\hline $\begin{array}{c}\text { Poster } \\
\text { Number }\end{array}$ & First Name & Last Name & $\begin{array}{l}\text { Abstract Title } \\
\text { Reliability and utility of a clip type electrode for recording an } \\
\text { electroencephalogram non-invasively from scalp hair }\end{array}$ \\
\hline 2 & Yasuhiko & Ajimi & $\begin{array}{l}\text { Noninvasive Continuous Imaging of Reduced Cerebral Perfusion with a } \\
\text { Novel Diffuse Optical Tomography System: A Preliminary Carotid Artery } \\
\text { Occlusion Study in Pig }\end{array}$ \\
\hline 3 & Chandran & Seshagiri & $\begin{array}{l}\text { pyMIND: Open-Source, Python-Based Multimodal Integrated Neural Data } \\
\text { Acquisition in the Neurointensive Care Unit }\end{array}$ \\
\hline 4 & Adewole & Oyalowo & \\
\hline
\end{tabular}

\section{Ischemic Stroke}

\begin{tabular}{|c|l|l|l|}
\hline $\begin{array}{c}\text { Poster } \\
\text { Number }\end{array}$ & First Name & Last Name & Abstract Title \\
\hline 5 & Neel & Singhal & $\begin{array}{l}\text { Resilience to Reperfusion injury in Arctic Ground Squirrel Neural Stem } \\
\text { Cells: Insights into novel pathways from a hibernating mammal }\end{array}$ \\
\hline 6 & Sankalp & Gokhale & $\begin{array}{l}\text { High Throughput Imaging of Motor System Connectivity in the Mouse } \\
\text { Brain. }\end{array}$ \\
\hline
\end{tabular}

\section{Other}

\begin{tabular}{|c|l|l|l|}
\hline $\begin{array}{c}\text { Poster } \\
\text { Number }\end{array}$ & $\begin{array}{l}\text { First } \\
\text { Name }\end{array}$ & Last Name & Abstract Title \\
\hline 7 & Eric & Marrotte & $\begin{array}{l}\text { Central Nervous System Extracellular Matrix Inhibition of Endothelial } \\
\text { Progenitor Cell Adhesion and Proliferation Can be Ameliorated with the } \\
\text { Use of a Novel Poly(Ethylene Glycol) Hydrogel. }\end{array}$ \\
\hline 8 & Polly & Pine & $\begin{array}{l}\text { Andexanet alfa but not Prothrombin Complex Concentrate Reverses } \\
\text { Rivaroxaban-induced Anticoagulation and Reduces Blood Loss in a } \\
\text { Rabbit Liver Laceration Model }\end{array}$ \\
\hline 9 & Jonathan & Elmer & $\begin{array}{l}\text { A randomized, crossover pilot trial of neuromonitoring-guided goal-direct } \\
\text { post-arrest care after opioid overdose }\end{array}$ \\
\hline
\end{tabular}

\section{Seizures}

\begin{tabular}{|c|l|l|l|}
\hline $\begin{array}{c}\text { Poster } \\
\text { Number }\end{array}$ & $\begin{array}{l}\text { First } \\
\text { Name }\end{array}$ & Last Name & Abstract Title \\
\hline 10 & Rebecca & Hammond & $\begin{array}{l}\text { Preclinical Pharmacology and Pharmacodynamic Interactions of SAGE- } \\
547: \text { Relevance to Super Refractory Status Epilepticus (SRSE). }\end{array}$ \\
\hline
\end{tabular}




\section{Subarachnoid hemorrhage}

\begin{tabular}{|c|l|l|l|}
$\begin{array}{c}\text { Poster } \\
\text { Number }\end{array}$ & $\begin{array}{l}\text { First } \\
\text { Name }\end{array}$ & Last Name & Abstract Title \\
\hline 11 & Jennifer & Kim & $\begin{array}{l}\text { Epileptiform Abnormalities and Delayed Cerebral Ischemia in } \\
\text { Subarachnoid Hemorrhage }\end{array}$ \\
\hline
\end{tabular}




\title{
Poster 1
}

\section{Cerebral blood flow thresholds for supply dependent glucose utilisation in traumatic brain injury: A multi-tracer 150xygen and [18F]-fluorodeoxyglucose positron emission tomography study}

\author{
Jeroen Hermanides ${ }^{1}$, Young Y. Hong ${ }^{2}$, Monica M. Trivedi ${ }^{1}$, Joanne J. Outtrim ${ }^{1}$, Franklin F. Aigbirhio ${ }^{2}$, \\ Peter P. Nestor ${ }^{3}$, Tim T. Fryer ${ }^{2}$, David D. Menon ${ }^{1}$, Jonathan J. Coles ${ }^{1}$.
}

${ }^{1}$ University of Cambridge, Division of Anaesthesia, Cambridge, United Kingdom (Great Britain), ${ }^{2}$ Wolfson Brain Imaging Centre, Department of Clinical Neurosciences, Cambridge, United Kingdom (Great Britain), ${ }^{3}$ German Center for Neurodegenerative Diseases, Magdeburg, Germany.

\section{Introduction:}

Metabolic derangements are common after traumatic brain injury (TBI), but few studies have used multitracer positron emission tomography (PET) to interrogate underlying pathophysiology. We examined relationships between glucose metabolism (measured using 18F-fluorodeoxyglucose (FDG) PET) and cerebral blood flow (CBF; measured using H215O PET).

\section{Methods:}

Twenty-six TBI patients with median (range) pre-intubation Glasgow Coma Score of 6 (3-12) underwent combined 150 and FDG PET on 34 occasions; 10 and 18 healthy volunteers (controls) underwent 150 and FDG PET respectively. FDG rate constants were determined with an irreversible two-compartment model: transport across BBB $(\mathrm{K} 1, \mathrm{k} 2)$, hexokinase activity $(\mathrm{k} 3)$, and influx rate $(\mathrm{Ki})$. Regions of interest (ROls) were defined for haemorrhagic lesion (core), hypodense tissue (penumbra), $1 \mathrm{~cm}$ border zone of normal appearing tissue (peri-penumbra), and remote normal appearing tissue (normal).

\section{Results:}

$\mathrm{K} 1$ was significantly reduced within core, but was comparable to control values in peri-penumbra and normal ROIs. While $\mathrm{k} 3$ and Ki were lower than control values, regional increases were found in the vicinity of lesions and some structurally normal areas. Glucose delivery (measured by K1) was unrelated to CBF when values were above $30 \mathrm{ml} / 100 \mathrm{ml} / \mathrm{min}$, and was significantly below $95 \%$ confidence intervals for control values when CBF was below $25 \mathrm{ml} / 100 \mathrm{ml} / \mathrm{min}$. There was a clear linear relationship between $\mathrm{K} 1$ and $\mathrm{CBF}$ in core ROls $\left(\mathrm{R}^{2}=0.75, \mathrm{p}<0.01\right)$. Ki exhibited similar relationships to $\mathrm{CBF}$, but showed more variability due to changes in $\mathrm{k} 3$.

\section{Conclusions:}

We found impaired FDG (glucose) transport within the vicinity of lesions that was linearly related to CBF below a threshold value of $30 \mathrm{ml} / 100 \mathrm{ml} / \mathrm{min}$. While a similar relationship was found for FDG influx (Ki), variability in $\mathrm{k} 3$ suggests different metabolic derangements are responsible. Further analyses will explore how such regional heterogeneity relates to evidence of necrosis, classical ischemia, and other metabolic derangements. 


\section{Poster 2}

\section{Reliability and utility of a clip type electrode for recording an electroencephalogram non- invasively from scalp hair}

Yasuhiko Ajimi ${ }^{1}$, Nana N. Oki ${ }^{2}$, Satoshi S. Hoshino ${ }^{2}$, Yuta Y. Oyama ${ }^{1}$, Takahiro T. Onuki ${ }^{1}$, Masahiro M. Snoo ${ }^{3}$, Tetsuya T. Sakamoto ${ }^{1}$.

${ }^{1}$ Teikyo University/Emergency Medicine, Tokyo, Japan, ${ }^{2}$ Teikyo University Hospital/Central Laboratory, Tokyo, Japan, ${ }^{3}$ Teikyo University/Neurology, Tokyo, Japan.

\section{Introduction:}

We created a clip type electrode (CE) for recording an electroencephalogram (EEG) non-invasively from scalp hair of normal volunteers and patients, and analyzed its reliability and utility.

\section{Methods:}

1) Reliability. (1) We compared interelectrode voltage (IV) and resistance (IR) between disc electrodes (DEs) and CEs. Each type were placed at the distance of one centimeter across paste (Elefix, EF, Nihon Kohden) for DEs or across preparation gel (Skinpure, SP, Nihon Kohden) for CEs. (2) We measured IR on the scalp from 12 normal volunteers under following conditions: (a) DEs with EF at F2 and P2 in the ten-twenty system, (b) DEs with EF after SP preparation, (c) CEs pinching scalp hair with SP at F1 and P1 and (d) CEs pinching scalp hair with SP after SP preparation. 2) Utility and Clinical application. EEGs were recorded by an electrode arrangement of $\mathrm{Fp} 1, \mathrm{Fp} 2, \mathrm{C} 3, \mathrm{C} 4, \mathrm{O} 1, \mathrm{O} 2$, T3 and T4 from ten normal volunteers and five ICU patients using Neurofax (Nihon Kohden). DEs were placed for right side recording and CEs were for left side.

\section{Results:}

1) Reliability. (1) IV/IR of DEs and CEs were $17 \mathrm{mV} / 1.1 \mathrm{k} \Omega$ and $24 \mathrm{mV} / 1.1 \mathrm{k} \Omega$. (2) Mean values (standard deviations) of scalp IR were (a) $263.6 \mathrm{k} \Omega$ (300.8), (b) $5.3 \mathrm{k} \Omega$ (5.3), (c) $130.3 \mathrm{k} \Omega$ (83.4) and (d) $7.4 \mathrm{k} \Omega$ (5.6). There were no significant differences between (a) and (b), and (c) and (d). 2) Utility and clinical application. Waveform analysis of EEGs recorded from ten volunteers and five ICU patients revealed no significant difference in amplitude and frequency spectrum between DEs and CEs. EEGs recording with CEs were stable and easy.

\section{Conclusions:}

Recording an EEG from scalp hair with CEs had the same reliability and utility to recording with DEs. 


\title{
Poster 3
}

\section{Noninvasive Continuous Imaging of Reduced Cerebral Perfusion with a Novel Diffuse Optical Tomography System: A Preliminary Carotid Artery Occlusion Study in Pig}

\author{
Chandran V. Seshagiri, Tanmayi T. Oruganti, Bertan B. Hallacoglu.
}

Research \& Development, Boston, MA, USA.

\section{Introduction:}

Diffuse optical tomography (DOT) systems have demonstrated the ability to non-invasively measure changes in oxy- and deoxy- hemoglobin concentrations in brain tissue. Cephalogics has introduced a novel, compact DOT system that may help clinicians monitor changes in brain perfusion and oxygenation following injuries such as ischemic stroke, vasospasm, or traumatic brain injury. In this study, we tested the ability of the DOT device to track changes following irreversible occlusion of the internal carotid arteries (ICAs) of a pig.

\section{Methods:}

A $41 \mathrm{~kg}$ Yorkshire pig was anesthetized (isoflurane) and prepared for surgery. The pig was placed in a supine position; a Cephalogics DOT sensor array was positioned under the pig's cranium; the array was secured using an elastic bandage wrap. Catheters were inserted into the femoral artery and advanced into both left and right ICAs just proximal to the retia mirabilia. Following a 15-minute baseline DOT recording, surgical glue was injected into both ICAs occluding the primary blood supply to the brain. DOT recording continued for 30 minutes following the occlusion, which was confirmed angiographically. DOT optical data were recorded to a laptop and images of the change in StO2 were reconstructed every 1 minute. We compared mean (+/-SD) StO2 across the entire imaged field (StO2-A) with the mean (+/-SD) $\mathrm{StO} 2$ in a regional area (StO2-R) associated with the pig's brain.

\section{Results:}

Comparing StO2 estimates pre- and post-occlusion, StO2-A showed a modest decrease while StO2- $\mathrm{R}$ decreased more significantly $(-3 \%+/-7 \%$ vs. $-15 \%+/-2 \%)$.

\section{Conclusions:}

Despite the thickness of the pig's skull, we captured a significant drop in StO2 following complete, irreversible occlusion of both ICAs. Due to the small size of the pig brain, the imaging field of the DOT sensor array includes both cerebral and non-cerebral tissue. The DOT images show a larger drop in StO2 in regions likely to reflect cerebral tissue. 


\title{
Poster 4
}

\section{pyMIND: Open-Source, Python-Based Multimodal Integrated Neural Data Acquisition in the Neurointensive Care Unit}

\author{
Adewole C. Oyalowo', Uday U. Agrawal' ${ }^{2}$, Andrew A. Pilling ${ }^{3}$, Shane S. Lee ${ }^{2}$, Wael W. Asaad ${ }^{4}$. \\ ${ }^{1}$ Brown University, NIH-Brown Graduate Partnership Program in Neuroscience, Providence, RI, USA, ${ }^{2}$ \\ Brown University, Department of Neuroscience, Providence, RI, USA, ${ }^{3}$ Brown University, Alpert Medical \\ School, Providence, RI, USA, ${ }^{4}$ Brown University and Rhode Island Hospital, Depts. of Neuroscience and \\ Neurosurgery, Providence, RI, USA.
}

\section{Introduction:}

Neurointensive care units (NeurolCUs) continuously monitor physiological vitals data from their patients in an attempt to predict and prevent secondary brain injury. However, only a sparse number of modern NeurolCUs are capable of continuously collecting and analyzing direct brain measures (e.g. electroencephalography (EEG) or intracranial pressure (ICP)). Additionally, severe limitations, such as infrequent and poorly standardized recording of the data, impair a physician's ability to track the progress of their patients over time or to observe potential relationships among the data streams. Furthermore, commercial solutions are often cost prohibitive, closed-source, and difficult to interface with. Here we present pyMIND, an open-source, Python-based program that addresses the problems of continuous data collection and analysis in the NeurolCU.

\section{Methods:}

pyMIND utilizes customized connection protocols to interface with and collect binary data from Philips Intellivue MP90 monitors and BrainVision EEG actiCHamp amplifiers via UDP, TCP/IP, and RS232 serial ports. Binary data parsers and storage protocols were created to extract the vitals and neural data from their various binary forms into a standardized, popular HDF5 format. Finally, graphical visualization tools were developed for real-time user interaction with the data.

\section{Results:}

An alpha version of pyMIND was developed that can reliably interface with, record from, and visualize data generated by demo modes of our target systems. The graphic user interface can plot trends of collected data (e.g. Arterial Blood Pressure, Pleth waveform), display head-montage power spectra of EEG data, and input time-stamped medical notes.

\section{Conclusions:}

pyMIND provides a flexible, open foundation to record and analyze the multitude of data streams collected in the NeuroICU. The real-time availability of vitals and neural data saved in a standardized format will allow researchers to conduct hypothesis-driven and machine learning-based analyses, which should help provide predictive indices of adverse events, enhance basic research in the NeurolCU, and improve patient care. 


\title{
Poster 5
}

\section{Resilience to Reperfusion injury in Arctic Ground Squirrel Neural Stem Cells: Insights into novel pathways from a hibernating mammal}

\author{
Neel S. Singhal ${ }^{1}$, Kayleigh K. Cook ${ }^{2}$, Dengke D. $\mathrm{Ma}^{2}$. \\ ${ }^{1}$ University of California-San Francisco, Dept. of Neurology, San Francisco, CA, USA, ${ }^{2}$ Cardiovascular \\ Research Institute, Department of Physiology, San Francisco, CA, USA.
}

\section{Introduction:}

Arctic ground squirrels (AGS) are extreme hibernators capable of withstanding months of freezing temperatures by suppressing metabolic rate. Hibernation is characterized by hypoxia and low cerebral blood flow and interrupted by bouts of arousal in which perfusion is quickly restored. Curiously, AGS do not experience reperfusion injury which is hypothesized to be reflected in altered transcriptional signatures in an in vitro model of reperfusion injury.

\section{Methods:}

To investigate the influence of ischemia/reperfusion on AGS neuronal stem and neural progenitor cells (NSC/NPCs), we exposed AGS and murine NSC/NPCs to control conditions, hypoxia, oxygen and glucose deprivation or glucose deprivation alone or following return to normal conditions to model reperfusion. Cell viability and cell cycle state were assessed by automated cytometry; metabolic phenotype by in vitro oxygen consumption and extracellular acidification rate. To determine novel genes involved in AGS resilience to reperfusion injury, a cDNA library was constructed in a mammalian expression vector and introduced into murine NSCs that were then assayed for viability after ischemia/reperfusion.

\section{Results:}

AGS NSC/NPCs demonstrated marked resistance to ischemia/reperfusion injury compared to murine NSC/NPCs. This survival phenotype is associated with suppressed mitochondrial oxidation and altered cell cycle regulation. AGS genes regulating mitochondrial function strongly modulated murine NSC/NPC viability following ischemia/reperfusion injury.

\section{Conclusions:}

A dynamic ability to suppress mitochondrial oxidation may underlie resilience to reperfusion injury in AGS by promoting a quiescent cell cycle phenotype. Development of therapeutic agents suppressing mitochondrial oxidation may induce a protective phenotype and promote survival following reperfusion injury. 


\title{
Poster 6
}

\section{High Throughput Imaging of Motor System Connectivity in the Mouse Brain.}

\author{
Sankalp Gokhale ${ }^{1}$, Katherine K. Poinsatte ${ }^{2}$, Shazia S. Mirza ${ }^{2}$, Denise D. Ramirez ${ }^{2}$, Xiangmei X. Kong ${ }^{2}$, \\ Erik E. Plautz", Mark M. Goldberg ${ }^{2}$.
}

${ }^{1}$ UT Southwestern, Department of Neurology and Neurotherapeutics, Dallas, TX, USA, ${ }^{2}$ UT Southwestern, Department of Neurology and Neurotherapeutics, Dallas, TX, USA.

\section{Introduction:}

Stroke results in profound alterations to architecture in the brain, particularly the corticospinal tract (CST). Some plasticity may contribute to functional recovery, while other changes may be maladaptive. Studies of CST connectivity have been limited by standard imaging methods which do not allow visualization and analysis of global axonal connectivity in the brain.

\section{Methods:}

We employed two novel imaging methods to visualize axonal projections to forelimb musculature. First, a pseudorabies viral (PRV) vector carrying green fluorescent protein (GFP) was injected into the left forelimb flexor in naïve 10-12 week-old C57 mice. PRV was transported retrogradely and transynaptically, labeling neurons in the motor cortex and other regions of interest. Whole slide imaging was performed using an automated slide scanner (Nanozoomer, Hamamatsu Photonics K.K., Hamamatsu City, Japan) producing images of serial coronal sections, allowing visualization of multiple levels of the brain in a single slide image. For serial two-photon tomography (STPT), UTSW Whole Brain Microscopy Facility used a TissueCyte 1000 imaging system (Tissue Vision, Somerville, MA) which uniquely performs automated sectioning and fluorescent imaging of the brain to produce 3-dimensional images with micronlevel resolution. This allows for unprecedented visualization of axonal connectivity in the whole brain.

\section{Results:}

We have imaged motor systems in the brain of uninjured mice using two different methods, each with distinct benefits. Whole slide imaging allows for quantification of regions of interest in the brain on a single slide, while STPT produces a highly detailed image that improves our understanding motor systems in 3D space.

\section{Conclusions:}

Future directions will investigate changes in connectivity following stroke injury and during recovery, allowing a greater understanding of the complexity of plasticity and how it contributes to beneficial and pathological circuit remodeling after injury. 


\title{
Poster 7
}

\section{Central Nervous System Extracellular Matrix Inhibition of Endothelial Progenitor Cell Adhesion and Proliferation Can be Ameliorated with the Use of a Novel Poly(Ethylene Glycol) Hydrogel.}

\author{
Eric J. Marrotte ${ }^{1}$, Ryan R. Schweller ${ }^{2}$, Jennifer J. West ${ }^{2}$, Daniel D. Laskowitz ${ }^{3}$.
}

${ }^{1}$ Duke University Medical Center/Department of Neurology, Durham, NC, USA, ${ }^{2}$ Duke University/Biomedical Engineering, Durham, NC, USA, ${ }^{3}$ Duke University Medical Center/Neurology, Durham, NC, USA.

\section{Introduction:}

We have previously found that mouse central nervous system extracellular matrix (CNS-ECM) inhibits endothelial progenitor cell (EPC) adhesion and proliferation. EPCs are circulating bone marrow derived cells that release neurotrophic growth factors and induce angiogenesis promoting enhanced perfusion to injured tissue. EPC adhesion and proliferation at the site of injury is necessary for EPC induced angiogenesis. In this study, we determine if a novel poly(ethylene glycol) (PEG) -based hydrogel developed to promote angiogenesis can be used to overcome impaired EPC adhesion and proliferation of EPCs on CNS-ECM.

\section{Methods:}

CNS-ECM was isolated from mouse brains and EPCs were isolated from mouse bone marrow. Cell culture wells were coated with CNS-ECM or CNS-ECM combined with a photopolymerized MMPcleavable PEG diacrylate hydrogel containing an RGDS binding sequence. Each well was then plated with 1x104 EPCs from the same cell population. An iCELLigence was used to measure EPC adhesion and proliferation which provide a curve for adhesion and proliferation. The curves for cell adhesion and proliferation comparisons were acquired over 2 hours and 50 hours, respectively, and compared.

\section{Results:}

Comparing EPCs plated on CNS-ECM vs. CNS-ECM with PEG-Hydrogel, EPC plated on CNS-ECM PEG-Hydrogel showed significant improvement in adhesion (adhesion slope: $0.033 \pm 0.018$ vs. $0.096 \pm$ 0.003 a.u., $\mathrm{P}<0.05, \mathrm{~N}=3$ ) and EPC proliferation (proliferation slope: $-0.0007 \pm 0.0004$ vs. $0.0090 \pm 0.0002$ a.u., $\mathrm{P}<0.05, \mathrm{~N}=3$ ). EPC plated on $\mathrm{PEG}-\mathrm{Hydrogel}$ alone (adhesion slope $0.069 \pm 0.001$ a.u., proliferation slope $0.0092 \pm 0.0001$ a.u.) showed an improvement in EPC proliferation vs. CNS-ECM $(P<0.05, N=3)$.

\section{Conclusions:}

Our data demonstrate for the first time that, inhibition of EPC adhesion and proliferation can be ameliorated with the use of a novel PEG hydrogel which promotes angiogenesis. The finding that a bioengineered material can be used to promote EPC adhesion and proliferation on CNS-ECM is an important step in the development of novel therapies for CNS regeneration. 


\title{
Poster 8
}

\section{Andexanet alfa but not Prothrombin Complex Concentrate Reverses Rivaroxaban-induced Anticoagulation and Reduces Blood Loss in a Rabbit Liver Laceration Model}

\author{
Polly Pine ${ }^{1}$, Genmin G. Lu', Francis F. DeGuzman ${ }^{2}$, Deogracias D. Canivel' ${ }^{2}$, John J. Malinowski ${ }^{2}$, \\ Pratikhya P. Pratikhya ${ }^{2}$, Joyce J. Lin ${ }^{2}$, Janet J. Leeds ${ }^{2}$, Stanley S. Hollenbach ${ }^{2}$, John J. Curnutte ${ }^{2}$, Pamela \\ P. Conley ${ }^{2}$. \\ ${ }^{1}$ Portola Pharmaceuticals, Inc., South San Francisco, CA, USA, ${ }^{2}$ Portola Pharmaceuticals, Inc., South \\ San Francisco, USA.
}

\section{Introduction:}

Andexanet alfa $(\mathrm{AnXa})$ is a modified recombinant factor $\mathrm{Xa}(\mathrm{FXa})$ derivative that sequesters direct $\mathrm{FXa}$ inhibitors and reverses their anticoagulation effects. Non-specific prothrombin complex concentrates (PCCs) have been proposed as potential reversal strategies. The objective of these studies was to compare 3-factor (Bebulin) and 4-factor (Kcentra $\left.{ }^{\circledR}\right)$ PCCs, approved for reversal of warfarin, with AnXa in reversing anticoagulation effects of rivaroxaban in a rabbit model of bleeding.

\section{Methods:}

NZW rabbits were treated with rivaroxaban (IV, $1 \mathrm{mg} / \mathrm{kg}$ ) and $30 \mathrm{~min}$ later, either PCCs (3-factor PCC: 60 or $120 \mathrm{mg} / \mathrm{kg}$; 4-factor PCC: 25,50 , or $100 \mathrm{IU} / \mathrm{kg}$ ) or AnXa (75 or $125 \mathrm{mg} / \mathrm{rabbit}$ ) was administered IV. Liver injury was then induced with $101-\mathrm{cm}$ incisions following laparotomy, and blood loss was measured for 15 min. Plasma concentrations of unbound (active) and total rivaroxaban, as well as pharmacodynamic (PD) parameters (anti-FXa activity, PT, and aPTT) were determined.

\section{Results:}

AnXa reduced blood loss in rivaroxaban-anticoagulated rabbits to levels seen in non-anticoagulated rabbits. In rabbits treated with AnXa, anti-FXa activity and unbound rivaroxaban were reduced dosedependently by $>95 \%$ and $>90 \%$, respectively, within 5 minutes, and both parameters correlated with reduction in blood loss. In contrast, 3-factor or 4-factor PCCs had no significant effect on any of these markers in rivaroxaban-anticoagulated rabbits.

\section{Conclusions:}

AnXa effectively reversed the anticoagulation activity of rivaroxaban in a rabbit model of bleeding. In contrast, PCCs showed no reversal activity as assessed by blood loss or PD markers. These results suggest that use of non-specific PCCs as reversal agents for direct FXa inhibitors are not likely to be as effective as specific reversal agents that target FXa inhibitors. Investigation of AnXa vs. PCC to reduce hematoma expansion in models of intracranial hemorrhage is warranted. 


\title{
Poster 9
}

\section{A randomized, crossover pilot trial of neuromonitoring-guided goal-direct post-arrest care after opioid overdose}

\author{
Jonathan Elmer ${ }^{1}$, Katharyn K. Flickinger ${ }^{2}$, Maighdlin M. Anderson ${ }^{3}$, Allison A. Koller ${ }^{2}$, Matthew M. \\ Sundermann ${ }^{2}$, Cameron C. Dezfulian ${ }^{3}$, David D. Okonkwo ${ }^{4}$, Lori L. Shutter ${ }^{5}$, David D. Salcido ${ }^{2}$, Clifton C. \\ Callaway ${ }^{2}$, James J. Menegazzi².
}

${ }^{1}$ Emergency Medicine and Critical Care Medicine, University of Pittsburgh, Pittsburgh, PA, USA, ${ }^{2}$ Emergency Medicine, Pittsburgh, PA, USA, ${ }^{3}$ Critical Care Medicine, Pittsburgh, PA, USA, ${ }^{4}$ Neurosurgery, Pittsburgh, PA, USA, ${ }^{5}$ Critical Care Medicine, Neurology and Neurosurgery, Pittsburgh, PA, USA.

\section{Introduction:}

Outcomes after resuscitation from cardiac arrest (CA) remain poor. Preventable secondary injury from ongoing brain tissue hypoxia (BTH) may worsen injury burden. Unfortunately, markers to allow individualized, real-time care optimization are lacking.

\section{Methods:}

We performed a randomized crossover trial in a swine model of opioid-induced CA to 1) determine the prevalence of BTH with standard care (StdC), and 2) test whether neuromonitor-guided goal-directed care (NGDC) can prevent BTH. Female swine $(25-30 \mathrm{~kg})$ were anesthetized with propofol and fentanyl. We placed femoral arterial and venous sheaths, a continuous cardiac output pulmonary artery catheter (Edwards LifeScience) and a right frontal intracranial access bolt (Hemedex) with probes for brain tissue oxygen (PbtO2), pressure (Raumedic), microdialysis (mDialysis70), cerebral blood flow (CBF) (Hemedex), and an 8-contact electroencephalographic depth electrode (AdTech). We induced apnea with $30 \mathrm{mcg} / \mathrm{kg}$ fentanyl, extubated the animal and began ACLS $9 \mathrm{~min}$ after apnea. After $1 \mathrm{~h}$ stabilization, animals with return of spontaneous circulation (ROSC) were randomized to three alternating $6 \mathrm{~h}$ care blocks: StdC (mean arterial pressure $>65 \mathrm{mmHg}$, oxygen saturation $94-98 \%$, cardiac output $>75 \%$ baseline) or NGDC (PbtO2>20 mmHg, CBF>20 mL $/ 100 \mathrm{~g} / \mathrm{min})$. Animals were euthanized at $18 \mathrm{~h}$ postROSC. Our primary outcome was the effect of care block on PbtO2, which we analyzed at $1 \mathrm{~min}$ resolution using generalized estimating equations with robust standard errors.

\section{Results:}

Overall, 8 of 17 animals achieved ROSC after $13 \pm 3$ min. PbtO2 was higher during NGDC than StdC $(P<0.001)$ and did not differ during NGDC from pre-arrest. PbtO2 was $<20 \mathrm{mmHg}$ more during StdC than NGDC $(40 \%$ of minutes vs $1 \%, P<0.001)$. CBF was lower during NGDC than StdC $(P<0.001)$, and lower in both arms than pre-arrest (both $\mathrm{P}<0.001$ ).

\section{Conclusions:}

Brain tissue hypoxia was common in this cardiac arrest model and prevented by neuromonitor-guided goal-directed care. Lower CBF and higher PbtO2 during goal-directed care implies preserved hypoxic cerebral vasodilation and diffusion-limited oxygen delivery. Future work will incorporate electroencephalographic and metabolic injury markers. 
Poster 10

\title{
Preclinical Pharmacology and Pharmacodynamic Interactions of SAGE-547: Relevance to Super Refractory Status Epilepticus (SRSE).
}

\author{
Rebecca S. Hammond ${ }^{1}$, Michael M. Ackley ${ }^{1}$, Michael M. Quirk ${ }^{1}$, Gabriel G. Belfort ${ }^{1}$, James J. Doherty ${ }^{2}$. \\ ${ }^{1}$ Drug Discovery and Development, Sage Therapeutics, Cambridge, MA, USA, ${ }^{2}$ Drug Discovery and \\ Development, Sage Theraputics, Cambridge, MA, USA.
}

\section{Introduction:}

SAGE-547 is a proprietary formulation of the endogenous neurosteroid allopregnanolone, being studied as a potential adjunctive therapy for the treatment of super-refractory status epilepticus (SRSE). SRSE refers to a condition of persistent seizures that have failed treatment with first-, second- and third-line treatments. In preclinical models, prolonged seizures reduce the surface expression of synaptic GABAA receptors, exacerbating neuronal excitability and limiting target sites for GABAergic treatments (eg. benzodiazepines). Here we present preclinical data describing the pharmacological properties of SAGE547 that support its further development as a potential treatment for SRSE.

\section{Methods:}

GABA-evoked currents were examined in vitro with whole cell patch clamp recordings in cells expressing recombinant $\alpha 1 \beta 2 \mathrm{~g} 2$ or $\alpha 4 \beta 3 \delta$ GABAA receptor subunits. The rat lithium-pilocarpine model of refractory status epilepticus (Pouliot 2013) was used to examine in vivo anticonvulsant activity. SAGE-547 or pentobarbital was administered intravenously 60 minutes after the onset of pilocarpine-induced seizures, a time point when benzodiazepines are ineffective in animal models (Pouliot 2013).

\section{Results:}

SAGE-547 potentiated both synaptic-type ( $\alpha 1 \beta 2 \mathrm{~g} 2)$ and extrasynaptic-type ( $\alpha 4 \beta 3 \delta)$ GABA currents in vitro, with EC50s of $60 \mathrm{nM}$ and $80 \mathrm{nM}$, respectively. The concentration-dependent potentiation of $\alpha 4 \beta 3 \delta$ GABAA receptors by SAGE-547 was 16,000 -fold more potent than that observed with pentobarbital alone. When SAGE-547 and pentobarbital were combined, the maximal potentiation of $\alpha 1 \beta 2 \mathrm{~g} 2$ and $\alpha 4 \beta 3 \delta$ currents was significantly increased in a synergistic manner. These combination effects were also observed in the rat model of RSE. When sub-active doses of SAGE-547 and pentobarbital were combined, electrographic seizure activity was significantly reduced.

\section{Conclusions:}

In vitro, SAGE-547 potently modulated both synaptic-type and extrasynaptic-type GABAA receptors, and the maximal potentiation at these receptors was further augmented by the co-application of pentobarbital. This enhanced in vitro potency and maximal effect at GABAA receptors provides further support for the development of SAGE-547 as a potential treatment for SRSE. 


\title{
Poster 11
}

\section{Epileptiform Abnormalities and Delayed Cerebral Ischemia in Subarachnoid Hemorrhage}

\author{
Jennifer A. Kim¹, Eric E. Rosenthal', Sydney S. Cash ${ }^{3}$, M. Brandon M. Westover ${ }^{3}$. \\ ${ }^{1}$ Massachusetts General Hospital Neurology, Boston, MA, USA, ${ }^{2}$ Massachusetts General \\ Hospita/Neurology, Boston, MA, USA, ${ }^{3}$ Massachusetts General Hospital/Neurology, Boston, MA, USA.
}

\section{Introduction:}

Vasospasm has long been considered the primary mechanism underlying delayed cerebral ischemia $(\mathrm{DCl})$ in subarachnoid hemorrhage (SAH), but increasing evidence shows that other processes such as cortical spreading depressions and inflammation. We propose that abnormal neural activity in the form of epileptiform abnormalities, we term ictal-interictal continuum abnormalities (IICAs), may contribute to DCl. These abnormalities may increase metabolic demands in injured brain tissue, thereby contributing to metabolic crisis and secondary neuronal injury. Here, we investigate whether the presence of IICAs predict $\mathrm{DCl}$ development.

\section{Methods:}

We analyzed EEG reports from 124 ICU patients with moderate-severe non-traumatic SAH. Continuous EEG data was recorded with daily review to identify electrographic seizures and interictal patterns. We tallied daily seizures, sporadic epileptiform discharges, lateralized or generalized periodic discharges (LPDs and GPDs), and lateralized or generalized rhythmic delta activity (LRDA and GRDA). Delayed cerebral ischemic events were also marked. Cumulative distribution curves and IICA-to-DCI time plots were calculated.

\section{Results:}

IICAs are more prevalent in patients who develop $\mathrm{DCl}$, especially when they begin several days after the onset of SAH. All IICA types except generalized rhythmic delta activity occur more commonly in patients who develop DCI. In particular, IICAs that begin later in hospitalization correlate with increased risk of DCI (LRDA day 9, ED day 6, LPDs day 12, GPDs day 5) Most IICAs also precede the onset of DCl. We next trend features of discharges to identify those most closely associated with $\mathrm{DCl}$ and will present our preliminary findings.

\section{Conclusions:}

IICAs represent a new marker for identifying early patients at increased risk for DCl. Moreover, IICAs might contribute mechanistically to $\mathrm{DCl}$ and therefore represent a new potential target for intervention to prevent secondary cerebral injury following SAH. 


\section{Clinical}

\section{All even numbered posters will present on Friday All odd numbered posters will present on Saturday All poster sessions are in Prince George Exhibit Hall A from 5:30pm-6:30pm}

\begin{tabular}{|c|c|c|c|}
\hline \multicolumn{4}{|c|}{ Disorders of Consciousness } \\
\hline $\begin{array}{l}\text { Poster } \\
\text { Number }\end{array}$ & First Name & Last Name & Abstract Title \\
\hline 12 & Prashanth & Krishnamohan & $\begin{array}{l}\text { Spectral Analysis of Continuous EEG for Outcome Prediction in Post- } \\
\text { Cardiac Arrest Coma }\end{array}$ \\
\hline 13 & Ariane & Lewis & $\begin{array}{l}\text { Public Education and Misinformation on Brain Death in Mainstream } \\
\text { Media }\end{array}$ \\
\hline 14 & Niel & Chen & $\begin{array}{l}\text { Establishing a Novel Classification System for Cardiac Arrest Etiology } \\
\text { to Predict the Course of Neurological Recovery }\end{array}$ \\
\hline 15 & Ali & Mahta & $\begin{array}{l}\text { Post-operative hemorrhage: a possible predictor of delirium in brain } \\
\text { tumor patients }\end{array}$ \\
\hline 16 & Terrance & Kummer & $\begin{array}{l}\text { EEG signal processing reveals features that differentiate structural } \\
\text { from non-structural disorders of consciousness }\end{array}$ \\
\hline 17 & D. Ethan & Kahn & $\begin{array}{l}\text { Resting EEG and Event Related Potentials in Outcome Prediction } \\
\text { Early in Severe Brain Injury }\end{array}$ \\
\hline 18 & Malik & Fakhar & $\begin{array}{l}\text { Optic Nerve Sheath Diameter On Brain CT After Cardiac Arrest Does } \\
\text { Not Correlate with Outcome }\end{array}$ \\
\hline
\end{tabular}

\begin{tabular}{|c|c|c|c|}
\hline \multicolumn{4}{|c|}{ Head and Spine Trauma } \\
\hline $\begin{array}{l}\text { Poster } \\
\text { Number }\end{array}$ & First Name & Last Name & Abstract Title \\
\hline 19 & Vijay & Krishnamoorthy & $\begin{array}{l}\text { Incidence and Longitudinal Trajectory of Early Systolic Dysfunction } \\
\text { After Traumatic Brain Injury }\end{array}$ \\
\hline 20 & Sophie & Samuel & $\begin{array}{l}\text { Detecting the Frequency and Severity of Paroxysmal Sympathetic } \\
\text { Hyperactivity Symptoms in Acute Traumatic Brain Injury }\end{array}$ \\
\hline 21 & Halinder & Mangat & $\begin{array}{l}\text { Hypertonic Saline Is Superior To Mannitol In The Combined Effects } \\
\text { On Intracranial Pressure And Cerebral Perfusion Pressure In Patients } \\
\text { With Severe Traumatic Brain Injury }\end{array}$ \\
\hline 22 & Claudia & Robertson & Phase II Clinical Trial of Atorvastatin in Mild Traumatic Brain Injury \\
\hline 23 & Tomoya & Okazaki & $\begin{array}{l}\text { Association between comorbidities, nutritional status, and anticlotting } \\
\text { drugs and neurological outcomes in geriatric patients with traumatic } \\
\text { brain injury }\end{array}$ \\
\hline 24 & Anthony & Tannous & $\begin{array}{l}\text { Serum Cytokine Levels Help Predict Critical Increases In Intracranial } \\
\text { Pressure }\end{array}$ \\
\hline 25 & Christopher & Melinosky & $\begin{array}{l}\text { Assessment of Autonomic Nervous System Dysfunction to Predict } \\
\text { Neuroworsening After Isolated Head Injury }\end{array}$ \\
\hline 26 & HALINDER & MANGAT & $\begin{array}{l}\text { Management of Severe Traumatic Brain Injury At a Tertiary Health } \\
\text { Center in Tanzania }\end{array}$ \\
\hline 27 & Brandon & Foreman, MD & $\begin{array}{l}\text { Bedside Stratification of Intracranial Pressure Elevations after Severe } \\
\text { Traumatic Brain Injury }\end{array}$ \\
\hline 28 & Andrew & Montoure & $\begin{array}{l}\text { Routine Repeat CT Head Is Not Necessary Without Neurological } \\
\text { Change For Mild Traumatic Brain Injury }\end{array}$ \\
\hline 29 & Thomas & Quinn & $\begin{array}{l}\text { Contrasting Preferences on the Communication of Prognosis between } \\
\text { Family Members and Physicians during Goals-of-Care Decisions in } \\
\text { Critically-III TBI Patients - Results from a Multi-Center Qualitative } \\
\text { Study }\end{array}$ \\
\hline 30 & Nasim & Ahmed & $\begin{array}{l}\text { IMPACT OF EMERGENCY DECOMPRESSIVE CRANIECTOMY } \\
\text { FOLLOWING BLUNT TRAUMATIC BRAIN INJURY ON MORTALITY: } \\
\text { PROPENSITY MATCHED ANALYSIS }\end{array}$ \\
\hline
\end{tabular}




\begin{tabular}{|c|c|c|c|}
\hline 31 & Brandon & Foreman, MD & $\begin{array}{l}\text { Bedside Detection of Spreading Depolarizations using Cortical Depth } \\
\text { Electrodes in Severe Non-Surgical Traumatic Brain Injury: Preliminary } \\
\text { Experience. }\end{array}$ \\
\hline 32 & Casey & Rommel & $\begin{array}{l}\text { IMPACT Traumatic Brain Injury Prognostic Calculations Use By } \\
\text { Clinicians: A Multidisciplinary, International Survey }\end{array}$ \\
\hline 33 & Sanjeev & Sivakumar & $\begin{array}{l}\text { Intensivist Practices in the Management of Severe Traumatic Brain } \\
\text { Injury: A Hemodynamic and Neuromonitoring Survey }\end{array}$ \\
\hline 34 & Susanne & Muehlschlegel & $\begin{array}{l}\text { Predicting Survival after Acute Civilian Penetrating Brain Injuries: The } \\
\text { SPIN Score }\end{array}$ \\
\hline 35 & Geert & Meyfroidt & $\begin{array}{l}\text { Real-time visualization of the cumulative pressure and time dose of } \\
\text { intracranial pressure in individual traumatic brain injured patients. }\end{array}$ \\
\hline 36 & Chitra & Sivasankar & $\begin{array}{l}\text { Acute Blood pressure variation and mortality in severe Traumatic } \\
\text { Brain injury }\end{array}$ \\
\hline 37 & SHUN & SUZUKI & $\begin{array}{l}\text { Relevance of Recurrence Rate and Air in Trepanation for Chronic } \\
\text { Subdural Hematoma }\end{array}$ \\
\hline 38 & Catalina & Sokoloff & $\begin{array}{l}\text { Trans-cranial Doppler as an Inference Tool of Cerebral Hypoxic } \\
\text { Episodes Early after Moderate and Severe TBI }\end{array}$ \\
\hline 39 & Nadia & Savy & $\begin{array}{l}\text { Computerized Classification of Brain Pathophysiological Status after } \\
\text { Severe Traumatic Brain Injury }\end{array}$ \\
\hline 40 & Andrew & Montoure & $\begin{array}{l}\text { Neuro-Critical Care Services Can Manage Mild Traumatic Brain Injury } \\
\text { Effectively Without Neurosurgical Consultation }\end{array}$ \\
\hline 41 & Sebina & Bulic & $\begin{array}{l}\text { Optic Nerve Sheath Diameter May Predict Elevations in Intracranial } \\
\text { Pressure }\end{array}$ \\
\hline 42 & Molly & Hubbard & $\begin{array}{l}\text { Elevated Intracranial Pressure Causes Reversible Eye Tracking } \\
\text { Changes Detected While Viewing Film Clip }\end{array}$ \\
\hline 43 & Jesse & Moskowitz & $\begin{array}{l}\text { Variable knowledge, use and perceptions of the IMPACT model } \\
\text { among physicians during prognostication meetings for critically-ill TBI } \\
\text { patients - results from a qualitative study }\end{array}$ \\
\hline 44 & Hiroyuki & Nakajima & $\begin{array}{l}\text { The Analyses of Acute Subdural Hematoma Using Logistic } \\
\text { Regression: The SIMC Experience }\end{array}$ \\
\hline 45 & Deepak & Jeswani & $\begin{array}{l}\text { Clinical Profile And Predictors Of In-Hospital Mortality In Traumatic } \\
\text { Brain Injuries: Urban Indian Experience }\end{array}$ \\
\hline
\end{tabular}

\section{ICU Organization and Technology}

\begin{tabular}{|c|c|c|c|}
\hline $\begin{array}{l}\text { Poster } \\
\text { Number }\end{array}$ & First Name & Last Name & Abstract Title \\
\hline 46 & Casey & Olm-Shipman & $\begin{array}{l}\text { Implementation of a Daily Goals Tool Improves Team Communication } \\
\text { Surrounding Quality and Safety Practices in a Neurosciences } \\
\text { Intensive Care Unit }\end{array}$ \\
\hline 47 & Jeannette & Hester & $\begin{array}{l}\text { Mission Impossible: Achieving Zero CAUTIs in the Neurolntensive } \\
\text { Care Unit Using a Patient- Centered, Interdisciplinary Team } \\
\text { Approach }\end{array}$ \\
\hline 48 & Yaw & Sarpong & $\begin{array}{l}\text { Improvement In Quality Outcome Measurements in Neurosciences } \\
\text { Intensive Care Unit After Appointment of Neurointensivists }\end{array}$ \\
\hline 49 & Bertan & Hallacoglu & $\begin{array}{l}\text { Noninvasive Continuous Imaging of Reduced Cerebral Perfusion with } \\
\text { a Novel Diffuse Optical Tomography System: A Preliminary } \\
\text { Hyperventilation Study in Pig and Human }\end{array}$ \\
\hline 50 & Brittany & Doyle & $\begin{array}{l}\text { Electronic (EPIC) Tool Facilitates Nurse Handover and Nurse-Led } \\
\text { Neurocritical Care Rounds }\end{array}$ \\
\hline 51 & Christa & Swisher & $\begin{array}{l}\text { The Use of Quantitative EEG Burst Suppression Ratio For Evaluating } \\
\text { Depth of Pharmacologic Coma }\end{array}$ \\
\hline 52 & Agnieszka & Ardelt & $\begin{array}{l}\text { Optimal Neurosciences Intensive Care Unit (Neuro - ICU) Staffing } \\
\text { Models: Addition of Night-time In-hospital Neurocritical Care Fellows } \\
\text { Correlates with Nursing, Physician, Non-physician Provider, and } \\
\text { Patient/Surrogate Satisfaction }\end{array}$ \\
\hline 53 & Paul & McCarthy & A Neurointensivist Managed Plasma Exchange Program \\
\hline 54 & Erika & $\begin{array}{l}\text { Schlichter, BSN } \\
\text { RN }\end{array}$ & $\begin{array}{l}\text { Outcome Following Acute Neurological Injury: A Pilot Feasibility } \\
\text { Study }\end{array}$ \\
\hline 55 & Michelle & Hill & $\begin{array}{l}\text { Staffing is More Than a Number: Using Workflow to Determine an } \\
\text { Appropriate Nurse Staffing Ratio in a Tertiary Care Neurocritical Care }\end{array}$ \\
\hline
\end{tabular}




\begin{tabular}{|c|l|l|l|}
\hline & & & Unit. \\
\hline 56 & Melissa & Panter & $\begin{array}{l}\text { Algorithm for Determining Venous Access Device in Neurocritical } \\
\text { Care }\end{array}$ \\
\hline 57 & Eric & Rosenthal & $\begin{array}{l}\text { A Conceptual Framework for a Technical Interoperability Standard } \\
\text { Promoting Highly Efficient Network-Based Clinical Trials and } \\
\text { Collaborative Research }\end{array}$ \\
\hline 58 & Shannon & Appelfeller & $\begin{array}{l}\text { Improving Patient Outcomes: The Impact of Creating a Specialized } \\
\text { Neuro-Oncology Critical Care Unit }\end{array}$ \\
\hline 59 & Anna & Rodriguez & $\begin{array}{l}\text { Recommendations for Medical Device Connectivity: Turning the NCS } \\
\text { Multimodal Monitoring Consensus Statement into Action }\end{array}$ \\
\hline
\end{tabular}

\section{Intracerebral hemorrhage}

\begin{tabular}{|c|c|c|c|}
\hline $\begin{array}{l}\text { Poster } \\
\text { Number }\end{array}$ & First Name & Last Name & Abstract Title \\
\hline 60 & Lauren & Peck & $\begin{array}{l}\text { Comparison of the Efficacy of 4-Factor and 3-Factor Prothrombin } \\
\text { Complex Concentrate for Reversing Warfarin in Patients with } \\
\text { Intracranial Hemorrhages }\end{array}$ \\
\hline 61 & Mario & Kofler & $\begin{array}{l}\text { Brain Temperature But Not Core Temperature Increases During } \\
\text { Cortical Spreading Depolarizations In Patients With Spontaneous } \\
\text { Intracerebral Hemorrhage }\end{array}$ \\
\hline 62 & Chandan & Mehta & Anticoagulation reversal: Time is of the Essence \\
\hline 63 & Sherri & Braksick & $\begin{array}{l}\text { Application of the FOUR Score in Intracerebral Hemorrhage Risk } \\
\text { Analysis }\end{array}$ \\
\hline 64 & Andrew & Naidech & $\begin{array}{l}\text { Depression Symptoms Are Associated with Worse Outcomes After } \\
\text { Intracerebral Hemorrhage and Are Under-Diagnosed Across Multiple } \\
\text { Healthcare Systems }\end{array}$ \\
\hline 65 & Jeffrey & Vitt & $\begin{array}{l}\text { The Impact of an Electronic Order Set on Time to Prothrombin } \\
\text { Complex Concentrate Administration for Vitamin-K Antagonist- } \\
\text { Associated Intracerebral Hemorrhage }\end{array}$ \\
\hline 66 & Andrew & Naidech & $\begin{array}{l}\text { The Spot Sign and Low Platelet Activity Identify Similar Patients at } \\
\text { Risk for Hematoma Growth }\end{array}$ \\
\hline 67 & Peter & Abdelmalik & $\begin{array}{l}\text { The Effect of Temperature on Clot Dissolution, and Outcomes, after } \\
\text { Spontaneous Intraventricular Hemorrhage: A Retrospective Analysis } \\
\text { of the CLEARIII-IVH Data }\end{array}$ \\
\hline 68 & Jamil & Dibu & $\begin{array}{l}\text { Prothrombin Complex Concentrates in Warfarin-Associated } \\
\text { Intracerebral Hemorrhage: Are We Underdosing? }\end{array}$ \\
\hline 69 & Nasim & Ahmed & $\begin{array}{l}\text { Prevalence of stroke following craniotomy or craniectomy for } \\
\text { spontaneously intracranial hemorrhage }\end{array}$ \\
\hline 70 & Ayham & $\begin{array}{l}\text { M. } \\
\text { Alkhachroum }\end{array}$ & $\begin{array}{l}\text { Neuroscience Intensive Care Unit Non-Admission Criteria for Patients } \\
\text { with Primary Intracerebral Hemorrhage }\end{array}$ \\
\hline 71 & Joseph & Blunck & $\begin{array}{l}\text { Evaluation of a Simplified 4-Factor Prothrombin Complex } \\
\text { Concentrate Dosing Protocol for the Reversal of Warfarin-Related } \\
\text { Intracranial Hemorrhage Patients }\end{array}$ \\
\hline 72 & Ayaz & Khawaja & $\begin{array}{l}\text { The Utility of Charlson Comorbidity Index in Predicting Outcomes in } \\
\text { Patients with Primary Intracerebral Hemorrhage }\end{array}$ \\
\hline 73 & Kathryn & Morbitzer & $\begin{array}{l}\text { Enhanced Renal Clearance in Patients with Intracerebral } \\
\text { Hemorrhage }\end{array}$ \\
\hline 74 & Neha & Dangayach & $\begin{array}{l}\text { Novel quantitative imaging markers for understanding cognitive } \\
\text { reserve in subdural hematoma (SDH) patients }\end{array}$ \\
\hline 75 & Christian & Hernandez & $\begin{array}{l}\text { Predictors of Surgical Intervention for Intracerebral Hemorrhage at a } \\
\text { High Volume Tertiary Medical Center }\end{array}$ \\
\hline 76 & Ayaz & Khawaja & $\begin{array}{l}\text { The Effect of Admission Systolic Blood Pressure on Mortality in } \\
\text { Patients with Acute Ischemic Stroke }\end{array}$ \\
\hline 77 & Johnnie & Dillinger & $\begin{array}{l}\text { Does NIH Stroke Scale Predict Length Of Stay (LOS) In Patients With } \\
\text { Intracranial Bleed (ICB)? }\end{array}$ \\
\hline 78 & Truman & Milling & $\begin{array}{l}\text { Treatment Patterns and Outcomes of Patients Receiving Oral Factor } \\
\text { Xa Inhibitors or Low Molecular Weight Heparin who Experienced Life- } \\
\text { threatening Intracranial Hemorrhage: A Retrospective Chart Review }\end{array}$ \\
\hline 79 & Andrew & Naidech & $\begin{array}{l}\text { Disparities in the Use of Seizure Medications for Patients with } \\
\text { Intracerebral Hemorrhage }\end{array}$ \\
\hline
\end{tabular}




\begin{tabular}{|c|c|c|c|}
\hline 80 & Anirudh & Sreekrishnan & $\begin{array}{l}\text { Greater gains in late recovery for intracerebral hemorrhage patients } \\
\text { with more debilitating initial injury. }\end{array}$ \\
\hline 81 & Audrey & Leasure & $\begin{array}{l}\text { Elderly Patients with Intracerebral Hemorrhage Have Higher } \\
\text { Morbidity than but Similar Mortality to Younger Patients at Three } \\
\text { Months. }\end{array}$ \\
\hline 82 & Jody & Manners & $\begin{array}{l}\text { High Variability in Acute Blood Pressure Control In Intracerebral } \\
\text { Hemorrhage }\end{array}$ \\
\hline 83 & Christopher & Robinson & $\begin{array}{l}\text { Intracerebral Hemorrhage and End-Stage Liver Disease: A Study of } \\
\text { Long Term Outcomes }\end{array}$ \\
\hline 84 & Denise & Rhoney & Assessment of Kcentra® Dosing Practices within Neurocritical Care \\
\hline 85 & Audrey & Leasure & $\begin{array}{l}\text { Perihematomal Edema Volume Predicts Poor Outcome at Discharge } \\
\text { in Patients with Cerebellar Intracerebral Hemorrhage. }\end{array}$ \\
\hline
\end{tabular}

\section{Ischemic Stroke}

\begin{tabular}{|c|c|c|c|}
\hline $\begin{array}{l}\text { Poster } \\
\text { Number }\end{array}$ & First Name & Last Name & Abstract Title \\
\hline 86 & Yahia & Lodi & $\begin{array}{l}\text { Primary Acute Stroke Thrombectomy within } 3 \text { Hours for Large Artery } \\
\text { Occlusion (PAST3-LAO) - A Pilot Study }\end{array}$ \\
\hline 87 & Christopher & Melinosky & $\begin{array}{l}\text { Early Prediction of Cerebral Herniation in Patients Presenting with } \\
\text { Large Hemispheric Infarction }\end{array}$ \\
\hline 88 & Rajat & Dhar & $\begin{array}{l}\text { CSF Volumetrics to Model Early Kinetics of Malignant Cerebral } \\
\text { Edema after Large Hemispheric Infarction }\end{array}$ \\
\hline 89 & Pitchaiah & Mandava & $\begin{array}{l}\text { Intravenous rt-PA Use Prior to Endovascular Thrombectomy } \\
\text { Increases Probability of Good Outcome in Acute Ischemic Stroke }\end{array}$ \\
\hline 90 & ABDULLAH & ALAMRI & $\begin{array}{l}\text { Safety and Efficacy of Mechanical Thrombectomy Under General } \\
\text { Anesthesia for Acute Ischemic Strokes }\end{array}$ \\
\hline 91 & Yekaterina & Axelrod & $\begin{array}{l}\text { Conservative initial management of young adults with severe } \\
\text { hemispheric stroke in a comprehensive stroke center reduces } \\
\text { decompressive craniectomy rates }\end{array}$ \\
\hline 92 & Yahia & Lodi & $\begin{array}{l}\text { Time of recanalization since symptoms is a strong predictor of } \\
\text { outcome in patients who underwent stent retriever thrombectomy from } \\
\text { middle cerebral artery occlusion. }\end{array}$ \\
\hline 93 & Jennifer & Frontera & $\begin{array}{l}\text { Infection and Stroke Risk following Continuous Flow Left Ventricular } \\
\text { Assist Device Implant }\end{array}$ \\
\hline 94 & Lais & Medeiros & $\begin{array}{l}\text { ENTERAL TUBE FEEDING IN ACUTE ISCHEMIC STROKE } \\
\text { PATIENTS: A MULTIVARIATE PREDICTIVE ANALYSIS. }\end{array}$ \\
\hline 95 & Terutake & Yonemori & $\begin{array}{l}\text { "Task Calc. Stroke" Tool: Managing IV t-PA Tasks Effectively for } \\
\text { Acute Stroke Patients }\end{array}$ \\
\hline 96 & Silvia & Schönenberger & $\begin{array}{l}\text { SEDATION AND AIRWAY DURING ENDOVASCULAR TREATMENT } \\
\text { OF ACUTE ISCHEMIC STROKE: PRIMARY ENDPOINT RESULTS } \\
\text { FROM THE RANDOMIZED TRIAL SIESTA (SEDATION VS } \\
\text { INTUBATION FOR ENDOVASCULAR STROKE TREATMENT) }\end{array}$ \\
\hline 97 & In Young & Chung & $\begin{array}{l}\text { Monitoring of Midline shift in Large Hemispheric Infarction during } \\
\text { Therapeutic Hypothermia ; As an Outcome predictor }\end{array}$ \\
\hline 98 & Yana & Bukovskaya & $\begin{array}{l}\text { Aminocaproic Acid for Reversal of Tissue Plasminogen Activator } \\
\text { (tPA) Related Hemorrhagic Transformation in Acute Ischemic Stroke. }\end{array}$ \\
\hline 99 & Sanjeev & Keshary & $\begin{array}{l}\text { Does Prior Use of Anti-Platelet Agents Matter for Endovascular } \\
\text { Therapy for Acute Ischemic Stroke and subsequent 3-Month } \\
\text { Functional Outcome? }\end{array}$ \\
\hline 100 & Virginia & Roberts & $\begin{array}{l}\text { The Effect of a Neurology-Specific Heparin Protocol with Narrowed } \\
\text { Goal PTT Range on Rate of Hemorrhagic and Thromboembolic } \\
\text { Events in Acute Ischemic Stroke: a Pre- and Post-Protocol } \\
\text { Implementation Study. }\end{array}$ \\
\hline 101 & $\begin{array}{l}\text { ANA } \\
\text { HELENA }\end{array}$ & ANDRADE & $\begin{array}{l}\text { THROMBOLYSIS INTRAVENOUS WITH ALTEPLASE IN ISCHEMIC } \\
\text { STROKE, BY SUPPORT NEUROLOGIST } \\
\text { TELEMEDICINA EXPERIENCE IN MOYSES DEUTSCH HOSPITAL }\end{array}$ \\
\hline
\end{tabular}


Medical Issues in NeurolCU

\begin{tabular}{|c|c|c|c|}
\hline $\begin{array}{l}\text { Poster } \\
\text { Number }\end{array}$ & First Name & Last Name & Abstract Title \\
\hline 102 & Megan & Barra & $\begin{array}{l}\text { Evaluation of Fibrinogen Concentrate (Riastap) for the Treatment of } \\
\text { Post-tPA Hemorrhage }\end{array}$ \\
\hline 103 & Casey & Olm-Shipman & $\begin{array}{l}\text { Impact of Implementing Team-Driven Interventions to Reduce } \\
\text { Catheter Associated Urinary Tract Infections in the Neuroscience } \\
\text { Intensive Care Unit }\end{array}$ \\
\hline 104 & Keshav & Goyal & $\begin{array}{l}\text { Non-Neurological Complications In Traumatic Neurosurgical ICU } \\
\text { Patients: A Prospective Observational Study }\end{array}$ \\
\hline 105 & Megan & Esch & $\begin{array}{l}\text { Transcranial Doppler in Patients Treated with Extracorporeal } \\
\text { Membrane Oxygenation: Changes in Pulsatility Index }\end{array}$ \\
\hline 106 & Kevin & Betthauser & $\begin{array}{l}\text { Practice Patterns Of Venous Thromboembolism Prophylaxis In } \\
\text { Underweight, Critically III Patients With Neurologic Injury }\end{array}$ \\
\hline 107 & Adam & Schertz & $\begin{array}{l}\text { Safety of Phenylephrine Infusion through Peripheral Intravenous } \\
\text { Catheter in Neuro Intensive Care Unit }\end{array}$ \\
\hline 108 & Christina & Roels & $\begin{array}{l}\text { A Retrospective Review of Combination Low Dose Mannitol and } \\
\text { Hypertonic Saline for the Treatment of Cerebral Edema }\end{array}$ \\
\hline 109 & Satoshi & Egawa & $\begin{array}{l}\text { Clinical characteristics of nonconvulsive status epilepticus diagnosed } \\
\text { by simplified continuous EEG monitoring at an emergency intensive } \\
\text { care unit. }\end{array}$ \\
\hline 110 & Naresh & Mullaguri & $\begin{array}{l}\text { Which Spontaneous Breathing Trial Predicts Liberation Success In } \\
\text { Neurocritical Care Patients? }\end{array}$ \\
\hline 111 & WANG & Rong & $\begin{array}{l}\text { Usage of a population pharmacokinetic model of vancomycin } \\
\text { in neurosurgical intensive care unit patients }\end{array}$ \\
\hline 112 & $\mathrm{Xi}$ & Liu-DeRyke & Effect of Clevidipine and Nicardipine on Blood Pressure Variability \\
\hline 113 & Megan & Garzon & $\begin{array}{l}\text { Prevalence of Ventricular Arrhythmias and Cardiac Arrest in patients } \\
\text { with Prolonged QT interval in Neuro ICU }\end{array}$ \\
\hline 114 & Amandeep & Dolla & $\begin{array}{l}\text { Prevalence of deep vein thrombosis among neuro-critical intensive } \\
\text { care unit patients }\end{array}$ \\
\hline 115 & Ariane & Lewis & $\begin{array}{l}\text { Prolonged Prophylactic Antibiotics with Neurosurgical Drains and } \\
\text { Devices: Are we using them? Do we need them? }\end{array}$ \\
\hline 116 & Abhijit & Lele & $\begin{array}{l}\text { Transition to Comfort Measures Only In Critically III Patients: Survey } \\
\text { Results from Health-Care Providers in a Large Academic Medical } \\
\text { Center }\end{array}$ \\
\hline 117 & Sarah & Adriance & Bradycardia with Dexmedetomidine in Neurocritically III Patients \\
\hline 118 & Gisele & Silva & Medical complications after subarachnoid hemorrhage. \\
\hline 119 & Ali & Saherwala & $\begin{array}{l}\text { Exploring Associations between Invasive and Non-Invasive Blood } \\
\text { Pressure Monitoring in Patients Receiving Vasoactive Medication } \\
\text { Infusions }\end{array}$ \\
\hline 120 & Satoshi & Egawa & Trend of New-onset Refractory Status Epilepticus (NORSE) in Japan \\
\hline 121 & Christopher & Morrison & $\begin{array}{l}\text { Intraventricular Colistin Use in Neurocritically III Patients with } \\
\text { Meningitis/Ventriculitis }\end{array}$ \\
\hline
\end{tabular}

\section{Muscle and Nerve Disorders}

\begin{tabular}{|c|l|l|l|}
\hline $\begin{array}{c}\text { Poster } \\
\text { Number }\end{array}$ & First Name & Last Name & Abstract Title \\
\hline 122 & Avinash & Kumar & $\begin{array}{l}\text { Myasthenic Crisis: epidemiology, economics and opportunities for } \\
\text { change - a single center retrospective analysis. }\end{array}$ \\
\hline 123 & Salvador & Cruz-Flores & Black tar heroin skin popping as a cause of botulism \\
\hline
\end{tabular}




\begin{tabular}{|c|c|c|c|}
\hline \multicolumn{4}{|l|}{ Other } \\
\hline $\begin{array}{l}\text { Poster } \\
\text { Number }\end{array}$ & First Name & Last Name & Abstract Title \\
\hline 124 & Karen & Lane & African American Enrollment in CLEAR III \\
\hline 125 & Edilberto & Amorim & $\begin{array}{l}\text { Dynamic quantitative EEG signatures predict outcome in cardiac } \\
\text { arrest }\end{array}$ \\
\hline 126 & Haroon & Majoka & $\begin{array}{l}\text { Impact of Secondary Brain Injury on Functional Outcome in ECMO- } \\
\text { treated Adults after Potentially Reversible Cardio-respiratory Failure }\end{array}$ \\
\hline 127 & Thorsten & Steiner & $\begin{array}{l}\text { Effect of Idarucizumab on Intracranial Hemorrhage in Dabigatran- } \\
\text { treated Patients: Initial Results from RE-VERSE AD }\end{array}$ \\
\hline 128 & Janet & Leeds & $\begin{array}{l}\text { The Effect of Andexanet Alfa on the Pharmacokinetics and Renal } \\
\text { Clearance of the Direct Factor Xa Inhibitors }\end{array}$ \\
\hline 129 & Callie & Drohan & $\begin{array}{l}\text { Sedation Use Alters Quantitative Electroencephalography in } \\
\text { Comatose Post-Cardiac Arrest Patients }\end{array}$ \\
\hline 130 & Ashley & Hedges & $\begin{array}{l}\text { Retrospective Analysis of Levetiracetam Dosing in Neurosurgical } \\
\text { Patients for Seizure Prophylaxis }\end{array}$ \\
\hline 131 & Alexandra & Baker & $\begin{array}{l}\text { Fibrinolytic For Treatment Of Intraventricular Hemorrhage: Meta } \\
\text { Analysis And Literature Review }\end{array}$ \\
\hline 132 & Kelly & Gassie & $\begin{array}{l}\text { Warfarin Subdural Hematoma (SDH) Re-Evacuation is More } \\
\text { Common than Antiplatelet Associated SDH }\end{array}$ \\
\hline 133 & Anna & $\begin{array}{l}\text { Finley Caulfield, } \\
\text { MD }\end{array}$ & $\begin{array}{l}\text { The Ability to Predict Long-term Outcome Differs Between Health } \\
\text { Care Provider Teams in Mechanically Ventilated Neurocritical Care } \\
\text { Patients }\end{array}$ \\
\hline 134 & Rachael & Schutz & $\begin{array}{l}\text { "Is There hope? Is She There?" - Perspectives Of Family Members, } \\
\text { Nurses, And Physicians Of Patients With Severe Acute Brain Injury }\end{array}$ \\
\hline 135 & Genmin & Lu & $\begin{array}{l}\text { Andexanet Alfa, an Investigational Universal Antidote for Reversal } \\
\text { of Anticoagulation of Factor Xa Inhibitors }\end{array}$ \\
\hline 136 & Fernando & Goldenberg & $\begin{array}{l}\text { Multicenter Observational Study of Early Mobilization in } \\
\text { Neurocritical patients: Implementation of a Protocol for Progressive } \\
\text { Mobility. }\end{array}$ \\
\hline 137 & Neha & Dangayach & $\begin{array}{l}\text { Critical Illness Resilience and Spirituality: Unique Methodology For } \\
\text { Surrogate Validation of CD-RISC-10 and Brief RCOPE Spirituality } \\
\text { Scales }\end{array}$ \\
\hline 138 & Fernando & Goldenberg & $\begin{array}{l}\text { Increasing Family Engagement in Neurocritical Care: A Qualitative } \\
\text { Study of Perceptions and Attitudes Regarding Passive Mobilization }\end{array}$ \\
\hline 139 & Anil & Ramineni & "I believe in miracles": Implications for the Neuro-ICU \\
\hline 140 & Mary & Barden & $\begin{array}{l}\text { Neurological Prognostication and Withdrawal of Life-Sustaining } \\
\text { Therapy (WLST) practices for Post-Cardiac Arrest Patients at a } \\
\text { Single Academic Medical Center: A Retrospective Analysis }\end{array}$ \\
\hline 141 & Swarna & Rajagopalan & $\begin{array}{l}\text { Invasive Multi-Modality Monitoring of Cerebral Physiology to Guide } \\
\text { Therapy in Hypoxic Ischemic Brain Injury }\end{array}$ \\
\hline 142 & Joseph & Haymore & $\begin{array}{l}\text { Implementation of a Validated Delirium Prediction Score in Patients } \\
\text { with Acute Cerebrovascular Disease }\end{array}$ \\
\hline 143 & David & Lerner & $\begin{array}{l}\text { Neurocritical care Education dUring Residency: OpinioNs } \\
\text { (NEURON) Study }\end{array}$ \\
\hline 144 & Aarti & Sarwal & $\begin{array}{l}\text { Institutionally Standardized Education Module \& EMR } \\
\text { documentation for Death by Neurological Criteria Evaluation can } \\
\text { improve Provider Compliance with Guidelines in a Tertiary Care } \\
\text { Medical Center }\end{array}$ \\
\hline 145 & Charles & Francoeur & $\begin{array}{l}\text { Evaluation of a Novel Noninvasive Cerebral Perfusion Monitor (C- } \\
\text { Flow) After Cardiac Arrest }\end{array}$ \\
\hline 146 & Alexander & Merkler & $\begin{array}{l}\text { Neurological Complications after Tuberculous Meningitis in the } \\
\text { United States }\end{array}$ \\
\hline 147 & Anastasia & Vishnevetsky & $\begin{array}{l}\text { Neuropalliative Care in Peru: Emergence from the Conspiracy of } \\
\text { Silence }\end{array}$ \\
\hline 148 & Kiruba & Dharaneeswaran & $\begin{array}{l}\text { Medical Training and the Brain Death Exam: A Single Institution's } \\
\text { Experience }\end{array}$ \\
\hline 149 & Aarti & Sarwal & $\begin{array}{l}\text { Pilot Study to Assess Quality of Communication in Acute } \\
\text { Neurological Emergencies and Define Domains for Improvement }\end{array}$ \\
\hline
\end{tabular}




\begin{tabular}{|c|l|l|l|}
\hline $\begin{array}{c}\text { Poster } \\
\text { Number }\end{array}$ & First Name & Last Name & Abstract Title \\
\hline 150 & Laith & Maali & $\begin{array}{l}\text { Cerebral Venous Thrombosis: Continental Characteristics and } \\
\text { Disparity }\end{array}$ \\
\hline 151 & Aarti & Sarwal & $\begin{array}{l}\text { Occurrence and Timeliness of Goals of Care Discussion in the } \\
\text { Neurocritical Care Unit }\end{array}$ \\
\hline 152 & SungEun & Lee & $\begin{array}{l}\text { Cerebral Vasomotor reactivity reflect prognosis after cardiac arrest } \\
\text { Can a Standardized Advanced Practice Provider (APP) Orientation } \\
\text { Process Transition New APP Skills Beyond the Level of the } \\
\text { Neuron? }\end{array}$ \\
\hline 153 & Michelle & Lozano & Early Evaluation of post-arrest injury and tailored TTM \\
\hline
\end{tabular}

\section{Pediatric NeurolCU}

\begin{tabular}{|c|l|l|l|}
\hline $\begin{array}{c}\text { Poster } \\
\text { Number }\end{array}$ & First Name & Last Name & Abstract Title \\
\hline 155 & Cydni & Williams & Endovascular Therapy in Pediatric Arterial Ischemic Stroke \\
\hline 156 & Luis & Lee & $\begin{array}{l}\text { Epidemiology And Outcomes Of Children With Diabetic Ketoacidosis } \\
\text { Presenting With Cerebral Edema }\end{array}$ \\
\hline 157 & Mullai & Baalaaji & $\begin{array}{l}\text { Correlation of Regional Cerebral Oxygenation with Cerebral } \\
\text { Perfusion Pressure in Children with Intracranial Hypertension due to } \\
\text { acute Central Nervous System (CNS) Infections - A Prospective } \\
\text { Observational Study }\end{array}$ \\
\hline 158 & Binod & Balakrishnan & $\begin{array}{l}\text { Enteral Nutrition Initiation in Children Admitted to the Pediatric } \\
\text { Intensive Care Unit after Traumatic Brain Injury }\end{array}$ \\
\hline 159 & Jennifer & Erklauer & $\begin{array}{l}\text { Outcome In Refractory and Super-Refractory Status Epilepticus In } \\
\text { Children }\end{array}$ \\
\hline 160 & Peter & Ferrazzano & $\begin{array}{l}\text { MRI Biomarkers of Functional Outcome after Severe Pediatric TBI: } \\
\text { A Feasibility Assessment }\end{array}$ \\
\hline
\end{tabular}

\section{Peri-Operative Management}

\begin{tabular}{|c|c|c|c|}
\hline $\begin{array}{l}\text { Poster } \\
\text { Number }\end{array}$ & First Name & Last Name & Abstract Title \\
\hline 161 & Andrea & Passarelli & $\begin{array}{l}\text { Antimicrobial prescribing practices and antibiotic resistance following } \\
\text { neurosurgical drain placement: A single-center observational study }\end{array}$ \\
\hline 162 & Lynn & Do & Levetiracetam for seizure prophylaxis after cerebrovascular surgery \\
\hline 163 & Jonathan & Rasouli & $\begin{array}{l}\text { Post-operative pain control after craniotomy: A meta-narrative } \\
\text { review }\end{array}$ \\
\hline 164 & Margaret & Huynh, DO & $\begin{array}{l}\text { Blood flow and cEEG changes during symptomatic plateau waves: } \\
\text { two cases }\end{array}$ \\
\hline
\end{tabular}

\section{Seizures}

\begin{tabular}{|c|l|l|l|}
\hline $\begin{array}{c}\text { Poster } \\
\text { Number }\end{array}$ & First Name & Last Name & Abstract Title \\
\hline 165 & Stephen & Kanes & $\begin{array}{l}\text { Hemodynamic properties of SAGE-547 in patients with super- } \\
\text { refractory status epilepticus: results from a phase 1/2 clinical trial }\end{array}$ \\
\hline 166 & Theresa & Human & $\begin{array}{l}\text { Duration of Seizure Prophylaxis after Subarachnoid Hemorrhage } \\
\text { (DOPAST): Results of a Randomized Comparative Effectiveness } \\
\text { Trial }\end{array}$ \\
\hline 167 & James & Braun & $\begin{array}{l}\text { Benzodiazepine Use For Status Epilepticus In An Academic Medical } \\
\text { Center Emergency Department }\end{array}$ \\
\hline 168 & Helen & Colquhoun & $\begin{array}{l}\text { Pharmacokinetics of SAGE-547 in patients with super refractory } \\
\text { status epilepticus }\end{array}$ \\
\hline 169 & Jennifer & Creed & $\begin{array}{l}\text { Early withdrawal of non-sedating antiepileptic drugs after successful } \\
\text { termination of nonconvulsive seizures and nonconvulsive status } \\
\text { epilepticus }\end{array}$ \\
\hline 170 & John & Faro & $\begin{array}{l}\text { Effect of Continuous Electroencephalography Coupled with } \\
\text { Aggressive Seizure Suppression on Outcomes after Cardiac Arrest }\end{array}$ \\
\hline
\end{tabular}




\begin{tabular}{|c|c|c|c|}
\hline $\begin{array}{l}\text { Poster } \\
\text { Number }\end{array}$ & First Name & Last Name & Abstract Title \\
\hline 171 & Lisa & Yanase & Phenytoin Dosing Adjustment for Obesity may not be Necessary \\
\hline 172 & Stephen & Kanes & $\begin{array}{l}\text { SAGE- } 547 \text { for the treatment of super-refractory status epilepticus: } \\
\text { response and relationship to underlying patient characteristics }\end{array}$ \\
\hline 173 & Jocelyn & Cheng & Withdrawal of Care and Its Impact on Mortality in Status Epilepticus \\
\hline 174 & Helen & Colquhoun & $\begin{array}{l}\text { Phase } 1 / 2 \text { open-label data suggest that heterogeneity of } \\
\text { presentation and high burden of comorbid illness do not impact the } \\
\text { activity of SAGE- } 547 \text { in patients with super-refractory status } \\
\text { epilepticus }\end{array}$ \\
\hline 175 & Michael & Mendoza & $\begin{array}{l}\text { Ictal-interictal Continuum (IICA) EEG patterns in Subarachnoid } \\
\text { Hemorrhage with Vasospasm }\end{array}$ \\
\hline 176 & Marta & Melis & $\begin{array}{l}\text { Non-Convulsive Seizures (NCS)/Non-Convulsive Status Epilepticus } \\
\text { (NCSE) in Neuro-ICU: Clinical Predictors and Prognostication }\end{array}$ \\
\hline 177 & Raquel & Farias-Moeller & $\begin{array}{l}\text { A Practical Approach to Ketogenic Diet in the Pediatric Intensive } \\
\text { Care Unit for Super Refractory Status Epilepticus }\end{array}$ \\
\hline 178 & Nirupama & Yechoor & $\begin{array}{l}\text { External Validation of the Epidemiology-Based Mortality Score in } \\
\text { Status Epilepticus Score in an American Population }\end{array}$ \\
\hline 179 & Chalita & Atallah & $\begin{array}{l}\text { Lost in translation: Survey of American Clinical Neurophysiology } \\
\text { Society (ACNS) standardized critical care electroencephalography } \\
\text { (EEG) terminology }\end{array}$ \\
\hline 180 & Pavel & Klein & Intravenous Carbamazepine: A New Formulation of a Familiar Drug \\
\hline
\end{tabular}

\section{Subarachnoid hemorrhage}

\begin{tabular}{|c|c|c|c|}
\hline $\begin{array}{l}\text { Poster } \\
\text { Number }\end{array}$ & First Name & Last Name & Abstract Title \\
\hline 181 & Daniel & Hänggi & $\begin{array}{l}\text { PHARMACOKINETICS AND HEALTH ECONOMICS OF } \\
\text { INTRAVENTRICULAR SUSTAINED RELEASE NIMODIPINE (EG- } \\
\text { 1962) FOR SUBARACHNOID HEMORRHAGE }\end{array}$ \\
\hline 182 & Robin & Bush & $\begin{array}{l}\text { Total Radiation Exposure during Initial Hospitalization in Patients } \\
\text { with Aneurysmal Subarachnoid Hemorrhage: A Retrospective } \\
\text { Observational Study }\end{array}$ \\
\hline 183 & Sungho & Ahn & $\begin{array}{l}\text { The Subarachnoid Hemorrhage Early Brain Injury Score (SEBIS) } \\
\text { Predicts Outcome after SAH }\end{array}$ \\
\hline 184 & Halinder & Mangat & $\begin{array}{l}\text { Biphasic Cerebral Blood Flow Response In Cerebral Hypoperfusion } \\
\text { After Aneurysmal Subarachnoid Hemorrhage }\end{array}$ \\
\hline 185 & Ranjita & Raghavan & $\begin{array}{l}\text { Trends in External Ventricular Drain Use, Ventriculostomy- } \\
\text { associated Infections, and Mortality in Aneurysmal Subarachnoid } \\
\text { Hemorrhage: Data from the Nationwide Inpatient Sample }\end{array}$ \\
\hline 186 & Mubashir & Pervez & $\begin{array}{l}\text { Effect of steroids on outcomes after aneurysmal subarachnoid } \\
\text { hemorrhage }\end{array}$ \\
\hline 187 & Nancy & Edwards & $\begin{array}{l}\text { Antiplatelet therapy for the prevention of peri-coiling } \\
\text { thromboembolism in high risk patients with ruptured intracranial } \\
\text { aneurysms }\end{array}$ \\
\hline 188 & Jin & Park & $\begin{array}{l}\text { Prediction of thrombotic complications and outcome in subarachnoid } \\
\text { hemorrhage patients using thromboelastography }\end{array}$ \\
\hline 189 & Muhammad & Athar & $\begin{array}{l}\text { Usefulness of Procalcitonin (PCT) to Differentiate between Infectious } \\
\text { and Central Fever in Patients with Subarachnoid Hemorrhage }\end{array}$ \\
\hline 190 & Imad & Khan & $\begin{array}{l}\text { Elevated Graeb Score is Associated With Loss of Independence } \\
\text { after Aneurysmal Subarachnoid Hemorrhage }\end{array}$ \\
\hline 191 & Jason & Siegel & $\begin{array}{l}\text { Usefulness of UltraSound Lung Comets as a Sign of Interstitial } \\
\text { Pulmonary Edema in Patients with High Grade Subarachnoid } \\
\text { Hemorrhage (US-LUNG-COMET Study) }\end{array}$ \\
\hline 192 & Nathaniel & Mohney & $\begin{array}{l}\text { Propensity Score Analysis of the Impact of Corticosteroid Use on } \\
\text { Delayed Cerebral Ischemia and Poor Functional Outcomes following } \\
\text { Subarachnoid Hemorrhage }\end{array}$ \\
\hline 193 & Sylvia & Bele & $\begin{array}{l}\text { Continuous intra-arterial infusion of Nimodipine (CIAN) for treatment } \\
\text { of severe refractory cerebral vasospasm (CV) after subarachnoid } \\
\text { hemorrhage (SAH)- early use of multimodal neuromonitoring is the } \\
\text { key for success }\end{array}$ \\
\hline
\end{tabular}




\begin{tabular}{|c|c|c|c|}
\hline $\begin{array}{l}\text { Poster } \\
\text { Number }\end{array}$ & First Name & Last Name & Abstract Title \\
\hline 194 & Charles & Francoeur & $\begin{array}{l}\text { Desmopressin for Prevention of Rebleeding in Subarachnoid } \\
\text { Hemorrhage patients }\end{array}$ \\
\hline 195 & David & Robinson & $\begin{array}{l}\text { Cardiac Arrest in Patients with Aneurysmal Subarachnoid } \\
\text { Hemorrhage: A Rare but Devastating Presenting Feature }\end{array}$ \\
\hline 196 & Tamara & Majic & $\begin{array}{l}\text { The effects of short versus longer duration prophylaxis of } \\
\text { levetiracetam on cognitive / functional outcomes in aneurysmal } \\
\text { subarachnoid hemorrhage and risk of development of delayed } \\
\text { seizures }\end{array}$ \\
\hline 197 & Robert & James & $\begin{array}{l}\text { Aneurysmal Subarachnoid Hemorrhage Trial Randomizing Heparin } \\
\text { (ASTROH): A Phase II Evaluation of Continuous Low-Dose } \\
\text { Intravenous Heparin Infusion for Low Grade aSAH Patients with } \\
\text { Large Hemorrhage Burden }\end{array}$ \\
\hline 198 & Manoj & Mittal & $\begin{array}{l}\text { Gastrointestinal Bleeding in Aneurysmal Subarachnoid Hemorrhage: } \\
\text { Incidence, Risk Factors, and Short-Term Outcomes }\end{array}$ \\
\hline 199 & Siddharth & Biswal & $\begin{array}{l}\text { Early Laboratory and Vital Sign Trends Predict Delayed Cerebral } \\
\text { Ischemia after SAH }\end{array}$ \\
\hline 200 & Sherry & Chou & $\begin{array}{l}\text { HIGH PLASMA MICRORNA-26A IS ASSOCIATED WITH GOOD } \\
\text { OUTCOME FOLLOWING SUBARACHNOID HEMORRHAGE }\end{array}$ \\
\hline 201 & Kathryn & Morbitzer & $\begin{array}{l}\text { Enhanced Renal Clearance in Patients with Aneurysmal } \\
\text { Subarachnoid Hemorrhage }\end{array}$ \\
\hline 202 & Mario & Kofler & $\begin{array}{l}\text { Enteral Feeding Increases Brain Interstitial Glucose Levels In Poor- } \\
\text { Grade Subarachnoid Hemorrhage Patients }\end{array}$ \\
\hline 203 & Michael & Reznik & $\begin{array}{l}\text { Agitation after subarachnoid hemorrhage: a frequent omen of } \\
\text { hospital complications associated with worse outcomes }\end{array}$ \\
\hline 204 & Sara & Hefton & $\begin{array}{l}\text { Extended Course of Levetiracetam is Associated with Loss of } \\
\text { Independence and Delirium after Aneurysmal Subarachnoid } \\
\text { Hemorrhage }\end{array}$ \\
\hline 205 & Matthew & Sharrock & $\begin{array}{l}\text { Intra-Aortic Balloon Pump Counterpulsation in patients with SAH and } \\
\text { Neurogenic Stunned Myocardium: A Case Series and Review of the } \\
\text { Literature. }\end{array}$ \\
\hline 206 & Eyad & Althenayan & $\begin{array}{l}\text { Use of Milrinone for the Management of Delayed Cerebral } \\
\text { Ischemia Following Non-Traumatic Sub-Arachnoid hemorrhage - A } \\
\text { Systematic Review }\end{array}$ \\
\hline 207 & Emmaculate & Fields & $\begin{array}{l}\text { Admission Mean Platelet Volume As A Predictor Of Subarachnoid } \\
\text { Hemorrhage Occurrence In Diabetics And Non-Diabetics }\end{array}$ \\
\hline 208 & Tomoya & Okazaki & $\begin{array}{l}\text { Blood lactate variability: a strong independent predictor of } \\
\text { neurological outcomes in patients with aneurysmal subarachnoid } \\
\text { hemorrhage }\end{array}$ \\
\hline 209 & Danyelle & Sadala & $\begin{array}{l}\text { Use of Milrinone in the Treatment of Delayed Cerebral Ischemia due } \\
\text { to Refractory Vasospasm: a Tertiary Academic Hospital Experience } \\
\text { in Brazil }\end{array}$ \\
\hline 210 & Sungho & Ahn & $\begin{array}{l}\text { Troponin Elevation for Multi-organ dysfunction in Subarachnoid } \\
\text { Hemorrhage }\end{array}$ \\
\hline 211 & sato & hiroki & $\begin{array}{l}\text { Postoperative management and outcome of aneurysmal } \\
\text { subarachnoid hemorrhage patients with surgery in Saitama medical } \\
\text { university international medical center -seven year survey- }\end{array}$ \\
\hline 212 & $\begin{array}{l}\text { Eva } \\
\text { Sahar }\end{array}$ & $\begin{array}{l}\text { Postma } \\
\text { Zafar }\end{array}$ & $\begin{array}{l}\text { Clinical Predictors of Outcomes in Patients with Aneurysmal } \\
\text { Subarachnoid Hemorrhage }\end{array}$ \\
\hline 213 & Apeksha & Shenoy* & $\begin{array}{l}\text { Dynamic Response of Neuronal Activity to Neurocritical Care } \\
\text { Interventions Measured by Intracranial EEG following Subarachnoid } \\
\text { Hemorrhage }\end{array}$ \\
\hline 214 & Kyle & Sheehan & $\begin{array}{l}\text { Delay in Presentation to Medical Care and Outcomes Following } \\
\text { Nontraumatic Subarachnoid Hemorrhage }\end{array}$ \\
\hline 215 & Hitoshi & Kobata & $\begin{array}{l}\text { Prophylactic Normothermia with Endovascular Cooling Device } \\
\text { Following Initial Therapeutic Hypothermia in Patients with Poor- } \\
\text { Grade Subarachnoid Hemorrhage }\end{array}$ \\
\hline 216 & Roy & Poblete & $\begin{array}{l}\text { Admission Lactic Acid Levels Are Associated With Clinical and } \\
\text { Radiographic Grade of Aneurysmal Subarachnoid Hemorrhage and } \\
\text { Predicts Inpatient Mortality }\end{array}$ \\
\hline
\end{tabular}




\section{Temperature Modulation}

\begin{tabular}{|c|l|l|l|}
\hline $\begin{array}{c}\text { Poster } \\
\text { Number }\end{array}$ & $\begin{array}{l}\text { First } \\
\text { Name }\end{array}$ & Last Name & Abstract Title \\
\hline 217 & Kyu Sun & Yum & $\begin{array}{l}\text { Factors associated with Failure of Targeted Temperature } \\
\text { Management on Malignant Hemispheric Infarction }\end{array}$ \\
\hline 218 & Hitoshi & Kobata & $\begin{array}{l}\text { Amplitude-Integrated EEG for Prognostication of Cardiac Arrest } \\
\text { Patients Under Extracorporeal Cardiopulmonary Resuscitation }\end{array}$ \\
\hline 219 & Richard & Riker & $\begin{array}{l}\text { Bedside Measurement of Suppression Ratio During Targeted } \\
\text { Temperature Management After Cardiac Arrest: Agreement } \\
\text { Between Medtronic Bispectral Index Monitor and Natus Full } \\
\text { Montage EEG Using Persyst Magic Marker Software }\end{array}$ \\
\hline 220 & Melissa & Naiman & $\begin{array}{l}\text { Temperature Management and Fever Avoidance in Critical Care } \\
\text { Patients using an Esophageal Heat Transfer Device }\end{array}$ \\
\hline 221 & Imad & Khan & $\begin{array}{l}\text { Novel Esophageal Cooling Device for Therapeutic Normothermia } \\
\text { Incidence, patterns, and risk factors of ischemic stroke after targeted } \\
\text { temperature management for cardiac arrest }\end{array}$ \\
\hline 222 & Yang-Je & Cho & $\begin{array}{l}\text { Performance of the EMCOOLs Surface Cooling System For Acute } \\
\text { Fever Control In Neurocritical Care Patients }\end{array}$ \\
\hline 223 & Stephen & Griffiths & \\
\hline & & & \\
\hline
\end{tabular}




\title{
Poster 12
}

\section{Spectral Analysis of Continuous EEG for Outcome Prediction in Post-Cardiac Arrest Coma}

\author{
Prashanth Krishnamohan, Babak B. Razavi, Kimford K. Meador, Karen K. Hirsch.
}

Stanford University, Department of Neurology, Stanford, CA, USA.

\section{Introduction:}

Up to $80 \%$ of patients resuscitated from cardiac arrest remain in a coma, and the ability to predict longterm neurologic recovery in these patients is limited. Quantitative analysis of electroencephalography (qEEG) is objective and may facilitate outcome prediction.

\section{Methods:}

Consecutive patients with hypoxic-ischemic coma were enrolled. Continuous EEG was obtained on all patients. EEG was post-processed and analyzed by Fourier transform. Spectral analysis was conducted on artifact-free contiguous 5-minute EEG epochs from each hour. Whole band $(1-30 \mathrm{~Hz})$, delta $(\delta, 1-4 \mathrm{~Hz})$ theta $(\theta, 4-8 \mathrm{~Hz})$ alpha $(\alpha, 8-13 \mathrm{~Hz})$, beta $(\beta, 13-30 \mathrm{~Hz})$ power, as well as $\alpha / \delta$ power and burst suppression ratio were computed as quantitative metrics of EEG for the entire EEG recording, and then statistically compared during the last 6 hours of EEG. Sedation, level of arousal, and body temperature were also analyzed. Good outcome (good neurologic outcome, GNO) was defined as consciousness recovery at any point in the acute hospitalization.

\section{Results:}

Ten subjects were included in the study, with cEEG durations ranging from $12-72$ hours of recording. The mean age was 49.6 years (18-65). There were significant differences in alpha power $(1.39$ (0.66 1.79 ) vs $0.27(0.17-0.48)$, median (IQR), $\mathrm{p}<0.05$, GNO vs poor neurologic outcome[PNO]), delta power (2.78 (2.21 - 3.01) vs 0.55 (0.38 - 0.83), median (IQR), $p=0.01$, GNO vs PNO), burst suppression ratio (0.66 (0.02-2.42) vs 73.4 (48.0 - 97.5), median (IQR), $p=0.01$, GNO vs PNO), and multiple measures of variability between GNO and PNO patients.

\section{Conclusions:}

Quantitative spectral analysis of continuous EEG may be predictive of consciousness recovery in patients with hypoxic-ischemic coma. Higher alpha power, lower burst suppression ratio, and higher variability were all correlated with good outcome. 


\title{
Poster 13
}

\section{Public Education and Misinformation on Brain Death in Mainstream Media}

\author{
Ariane Lewis ${ }^{1}$, Aaron A. Lord ${ }^{1}$, Barry B. Czeisler ${ }^{1}$, Arthur A. Caplan ${ }^{2}$. \\ ${ }^{1}$ NYU Langone Medical Center, Departments of Neurology and Neurosurgery, New York, NY, USA, ${ }^{2}$ \\ NYU Langone Medical Center, Department of Population Health, New York, NY, USA.
}

\section{Introduction:}

Because the media plays an important role in educating the public and impacting public perception on medical topics, we sought to evaluate whether mainstream media provides education or misinformation to the public about brain death through review of articles on two recent highly publicized brain death cases: 1) the Jahi McMath case, in which a teenage girl was declared brain dead and her family refused to allow organ support to be discontinued; and 2) the Marlise Muñoz case, in which a pregnant woman was declared brain dead and the hospital refused to terminate organ support until they were ordered to do so by a judge.

\section{Methods:}

We reviewed articles published prior to July 31,2015 on the most shared/heavily trafficked mainstream media websites of 2014 using the search terms, "Jahi McMath" and "Marlise Muñoz." Each article was evaluated to determine whether it contained 1) teaching points, or 2) misinformation, defined as misleading, incomplete, or incorrect information.

\section{Results:}

We reviewed 208 unique articles. The subject was referred to as being "alive" or on "life support" in $72 \%$ (149) of the articles, $97 \%$ (144) of which also described the subject as being brain dead. A definition of brain death was provided in $4 \%$ (9) of the articles. Only $7 \%$ (14) of the articles noted that organ support should be discontinued after brain death declaration unless a family has agreed to organ donation. Reference was made to well-known cases of patients in persistent vegetative states in $16 \%$ (34) of articles and $47 \%$ (16) of these implied both patients were in the same clinical state.

\section{Conclusions:}

Mainstream media provides poor education to the public on brain death. Because public understanding of brain death impacts organ and tissue donation, it is important for physicians, organ procurement organizations, and transplant coordinators to improve public education on this topic. 


\title{
Poster 14
}

\section{Establishing a Novel Classification System for Cardiac Arrest Etiology to Predict the Course of Neurological Recovery}

\author{
Niel L. Chen ${ }^{1}$, Cameron C. Dezfulian ${ }^{2}$, Jon J. Rittenberger ${ }^{3}$, Ankur A. Doshi ${ }^{3}$, Francis F. Guyette ${ }^{3}$, Clifton \\ C. Callaway ${ }^{3}$, Jonathan J. Elmer ${ }^{4}$. \\ ${ }^{1}$ University of Pittsburgh School of Medicine, Pittsburgh, PA, USA, ${ }^{2}$ University of Pittsburgh/Critical Care \\ Medicine, Pittsburgh, PA, USA, ${ }^{3}$ University of Pittsburgh/Emergency Medicine, Pittsburgh, PA, USA, \\ ${ }^{4}$ University of Pittsburgh/Emergency Medicine, Critical Care Medicine, Pittsburgh, PA, USA.
}

\section{Introduction:}

Classification of the etiology of cardiac arrest (CA) is difficult. Simply separating "presumed cardiac" from other etiologies may overlook important patient heterogeneity. We developed a comprehensive CA etiology classification system and compared outcomes across categories.

\section{Methods:}

We defined CA etiologies and diagnostic criteria through a modified Delphi process, engaging emergency physicians, cardiologists, neuro-intensivists and ICU nurses. We performed a structured chart review of consecutive patients admitted to a single center after CA from 2012-2015. A random subset of $20 \%$ of charts were double-reviewed to determine inter-rater reliability. We assigned arrest etiology, and recorded illness severity (Pittsburgh Cardiac Arrest Category), arrest location, initial rhythm, and outcomes (discharge location, Cerebral Performance Category (CPC), and time to awakening). We compared discharge location (home/acute rehabilitation vs. other) using unadjusted and adjusted logistic regression, CPC using ordered logistic regression, and time to awakening using a generalized linear model with a Poisson distribution. For this initial analysis, we grouped related etiology subtypes into broader categories then tested for heterogeneity within these categories.

\section{Results:}

Our final system included $17 \mathrm{CA}$ etiologies that grouped into 5 broad categories. Among 632 subjects, survival was $37 \%$ and $23 \%$ were discharged to home or rehabilitation. Broad categories were cardiac $(24 \%)$, toxicological $(10 \%)$, respiratory failure $(20 \%)$, other $(17 \%)$, and no identifiable etiology $(28 \%)$. Inter-rater reliability was high (kappa=0.82). In adjusted analysis, survival, discharge location, and CPC were better for the cardiac etiologies than all other broad categories (all adjusted $\mathrm{Ps}<0.001$ ). Compared to cardiac etiologies, time to awakening was significantly longer for toxicological causes $(+3 d, P<0.001)$, shorter for other and unknown causes (both $-0.8 \mathrm{~d}$; $\mathrm{P}<0.01$ ), and not different for respiratory causes $(\mathrm{P}=\mathrm{NS})$. There was no significant heterogeneity in outcomes between etiologies within broad categories.

\section{Conclusions:}

Using a structured chart review, $72 \%$ of hospitalized CA patients had identifiable CA etiologies that were associated with significant outcome variation. 


\title{
Poster 15
}

\section{Post-operative hemorrhage: a possible predictor of delirium in brain tumor patients}

\author{
Ali Mahta ${ }^{1}$, Ali A. Mahta ${ }^{1}$, Michael M. Reznik ${ }^{1}$, Soojin S. Park ${ }^{1}$, Sachin S. Agarwal ${ }^{1}$, David D. Roh ${ }^{1}$, Guy \\ G. McKhann ${ }^{2}$, Andrew A. Lassman ${ }^{3}$, Jan J. Claassen ${ }^{1}$. \\ ${ }^{1}$ Columbia University/Division of Critical Care Neurology, New York, NY, USA, ${ }^{2}$ Columbia \\ University/Department of Neurological Surgery, New York, NY, USA, ${ }^{3}$ Columbia University/Herbert Irving \\ Comprehensive Cancer Center, New York, NY, USA.
}

\section{Introduction:}

Post-operative delirium after brain tumor resection is frequent, difficult to manage, and may increase length of stay. We sought risk factors of agitated delirium after brain tumor resection through retrospective chart review of patients admitted to the Neurologic Intensive Care Unit (NICU) after brain tumor resection. We also evaluated the effect of agitated delirium on length of stay.

\section{Methods:}

Medical records of NICU admissions form 2013-2015 were reviewed to identify cases and controls. Cases were defined as patients with no pre-existing neuropsychiatric history who experienced significant agitated delirium post-operatively, defined by requirement for treatment with neuroleptics (quetiapine or dexmedetomidine) $<48$ hours after surgical resection. We compared these patients to a control group comprised of randomly selected patients admitted after brain tumor resection who did not experience agitated delirium. In a multi-variate logistic regression model, we explored the following hypothetical risk factors: sex, age, tumor location, pathology, post-operative stroke, clinical or electrographic seizures, post-operative hemorrhage, use of steroids and prophylactic anti-epileptics, particularly levetiracetam.

\section{Results:}

There were 48 cases and 49 controls. Multivariate analysis revealed male sex (O.R $3.47 ; 95 \% \mathrm{Cl} 1.17-$ 10.32; $p=0.025$ ) and a post-operative course complicated by hemorrhage within the resection cavity (O.R $10.13 ; 95 \% \mathrm{Cl} 3.27-31.64 ; p<0.001)$ as significant predictors of agitated delirium. The ICU length of stay was significantly longer in those with agitated delirium $(6.3 \pm 5.1$ days vs. $1.8 \pm 1.4$ days; $p<0.001)$.

\section{Conclusions:}

Neurointensivists caring for post-operative patients with brain tumor may consider resection site hemorrhage as a risk factor for developing agitated delirium. Future studies may investigate electrographic seizures as the possible link between post- operative bleeding and delirium and the long term outcome of these patients. 
Poster 16

EEG signal processing reveals features that differentiate structural from non-structural disorders of consciousness

Terrance T. Kummer ${ }^{1}$, Shoko S. Ryu ${ }^{2}$, Debra D. Roberts ${ }^{3}$, Lawrence L. Eisenman ${ }^{1}$, ShiNung S. Ching ${ }^{4}$.

${ }^{1}$ Washington University School of Medicine in St. Louis/Neurology, Saint Louis, MO, USA, ${ }^{2}$ Washington University/ Electrical and Systems Engineering, Saint Louis, MO, USA, ${ }^{3}$ University of Rochester Medical Center/Neurosurgery, Rochester, NY, USA, ${ }^{4}$ Washington University/Electrical and Systems Engineering , Saint Louis, MO, USA.

${ }^{* * * *}$ Permission was not granted to print this abstract ${ }^{* \star * *}$ 


\section{Poster 17}

\section{Resting EEG and Event Related Potentials in Outcome Prediction Early in Severe Brain Injury}

D. Ethan Kahn ${ }^{1}$, Bryan B. Taylor ${ }^{2}$, Peter P. Lyons ${ }^{3}$, Annie A. Tran ${ }^{3}$, Angela A. Herman ${ }^{4}$, John J. Getchell, Valerie V. Dechant ${ }^{6}$, Richard R. Choi ${ }^{7}$, Jennifer J. Miller ${ }^{6}$, James J. Hoffman ${ }^{3}$.

${ }^{1}$ Neurocritical Care, Christiana Care Health System, Newark, DE, USA, ${ }^{2}$ Value Institute, Christiana Care Health System, Newark, DE, USA, ${ }^{3}$ University of Delaware Department of Psychology, Newark, DE, USA, ${ }^{4}$ Department of Heart and Vascular, Christiana Care Health System, Newark, DE, USA, ${ }^{5}$ Department of Surgery, Christiana Care Health System, Newark, DE, USA, ${ }^{6}$ Department of Neurocritical Care, Christiana Care Health System, Newark, DE, USA, ${ }^{7}$ Department of Neurocrititcal Care, Christiana Care Health System, Newark, DE, USA.

\section{Introduction:}

Predicting outcome in the acute setting of severe brain injury (SBI) remains limited. Additional studies, particularly those which are noninvasive and readily obtainable, may assist with this. Event related Potentials (ERPs) are task-driven phenomena where individuals are given a stimulus, and time-locked components on electroencephalogram (EEG) are evaluated. Resting EEG ( $r E E G$ ) derives the power /distribution of specific EEG frequencies from an unstimulated individual. We speculated that these measures are feasible early in SBI and potentially predictive of outcome.

\section{Methods:}

The cohort consisted of subjects with SBI aged 18-80 years and a Glasgow Coma Scale (GCS) of 3-7 at day 4-21 post-injury. Outcome measures included: GCS, revised coma recovery scale (CRS-r) at discharge, and Glasgow Outcome Score Extended (GOSE) at 6 months. Positive outcome was defined as GOSE 4. ERP P300: Subjects were assessed with the Oddball paradigm (tones with deviant interruptions) and Subjects Own Name (SON) paradigm (a series of random names, including the patient's own name). Resting EEG: Recorded over 30-minutes. One-hundred 4-second epochs were selected per subject. Frequency bands of interest (delta, theta, alpha) underwent Fast Fourier Transformation. Values were averaged and plotted per trial.

\section{Results:}

From 4/15/15-5/15/16, 7 subjects were enrolled. Testing was performed at a mean of 5 days post injury. 4/7 subjects died before discharge, 3/7 survived. CRS-r on discharge: 1 (coma), 17 (minimally conscious), 17. At 6 months, GOSE $=1,4,4$ (death, upper severe disability). Both survivors had robust P300 components and alpha power (AP). Decedents had no P300 components and low AP; none recovered consciousness before natural death or withdrawal of care. A posterior dominant AP on rEEG was demonstrated in both survivors and no decedents

\section{Conclusions:}

Early acquisition of ERP and rEEG measures in the acute setting of SBI is feasible. Although our sample is small, posterior dominant AP and P300 are promising indicators of positive outcome. 


\title{
Poster 18
}

\section{Optic Nerve Sheath Diameter On Brain CT After Cardiac Arrest Does Not Correlate with Outcome}

\author{
Malik Fakhar ${ }^{1}$, Nikita N. Dave ${ }^{2}$, Xian X. Wu ${ }^{3}$, Linda L. Gerber ${ }^{3}$, Santosh S. Murthy ${ }^{1}$, Oren O. Friedman ${ }^{4}$,
} Halinder H. Mangat ${ }^{5}$.

\footnotetext{
${ }^{1}$ Weill Cornell Medicine / Neurology, New York, NY, USA, ${ }^{2}$ The College of New Jersey, Ewing, NJ, USA, ${ }^{3}$ Weill Cornell Medicine / Healthcare Policy And Research, New York, NY, USA, ${ }^{4}$ Cedars-Sinai Medical Center / Internal Medicine - Critical Care, Los Angeles, CA, USA, ${ }^{5}$ Weill Cornell Medicine / Neurology \& Neurosurgery, New York, NY, USA.
}

\section{Introduction:}

Cardiac arrest is a leading cause of death and disability, and predicting outcome in these patients is a challenge. Optic nerve sheath diameter (ONSD) on brain CT correlates closely with intracranial pressure. Therefore in this study we studied correlation between ONSD measured on the initial brain CT in patients after cardiac arrest and outcome.

\section{Methods:}

This is a retrospective study of patients with cardiac arrest admitted to the medical intensive care unit at our hospital between 2009 and 2014. Patients who underwent brain CT in the first week after cardiac arrest were included. Demographics, neurological status on arrival and day 14 and outcomes were collected. ONSD on brain CT was measured bilaterally $3 \mathrm{~mm}$ behind the optic nerve head and averaged for each patient.

\section{Results:}

A total of 71 patients were included. Mean age was $66 \pm 13.6$ years, 54 patients $(76 \%)$ were male. 46 patients (65\%) suffered out-of-hospital cardiac arrest. Mean Glasgow Coma Scale (GCS) on admission was 4.35 \pm 1.96 (range 3-9; median 3). Return of spontaneous circulation (ROSC) time was 15.63 \pm 9.37 minutes. 35 patients $(49 \%)$ had a shockable rhythm and $16(22.3 \%)$ underwent percutaneous cardiac intervention. 70 patients $(98.59 \%)$ underwent therapeutic hypothermia. 17 patients $(23.9 \%)$ had seizures. Average modified Rankin Scale (mRS) at discharge was 4.74 $\pm 1.90,45$ patients $(63.38 \%)$ died while 14 $(19.7 \%)$ had a good outcome (mRS 0-3). Average duration from ROSC to CT was $1.3 \pm 2.04$ days. Mean ONSD in patients with GCS 3-8 at day 14 was $5.76 \pm 0.69 \mathrm{~mm}$, while in those with GCS 9-15 at day 14 , ONSD was $5.81 \pm 0.72 \mathrm{~mm}(\mathrm{p}=0.78)$. Mean ONSD in patients with $\mathrm{mRS} 0-3$ at discharge was $5.56 \pm 0.62 \mathrm{~mm}$, while in those with $\mathrm{mRS} 4-6$ was $5.88 \pm 0.71(p=0.28)$.

\section{Conclusions:}

There does not appear to be a significant correlation between the ONSD on brain CT in first week after cardiac arrest and outcome at day 14. 


\section{Poster 19}

\section{Incidence and Longitudinal Trajectory of Early Systolic Dysfunction After Traumatic Brain Injury}

Vijay Krishnamoorthy ${ }^{1}$, Edward E. Gibbons ${ }^{2}$, Ali A. Rowhani-Rahbar ${ }^{3}$, Kevin K. Luk ${ }^{1}$, Monica M. Vavilala ${ }^{1}$.

${ }^{1}$ Anesthesiology and Pain Medicine, Seattle, WA, USA, ${ }^{2}$ Medicine, Division of Cardiology, Seattle, WA, USA, ${ }^{3}$ Epidemiology, Seattle, WA, USA.

\section{Introduction:}

Traumatic brain injury (TBI) is a major public health problem. While the association between subarachnoid hemorrhage and systolic dysfunction (SD) has been established, the effect of TBI on cardiac function is unknown. Our study's aims were to 1) Determine the incidence of early SD in previously healthy patients following moderate-severe TBI, and 2) To describe the longitudinal change in systolic function over the first week of hospitalization.

\section{Methods:}

We conducted a prospective cohort study among mild and moderate-severe TBI patients admitted to a level 1 trauma center with these inclusion criteria: 1) Age < 65 years, 2) No severe non-TBI injuries, 3) No prior cardiac disease, and 4) Minimal comorbidities. Transthoracic echocardiograms were performed at $<24$ hours, 3-4 days, and 7-9 days following TBI. Systolic function was assessed using fractional shortening (FS), and SD was defined as FS $<25 \%$. Descriptive statistics were used to compare the mild and moderate-severe TBI groups. Multivariable linear regression was used to compare fractional shortening between groups.

\section{Results:}

64 patients were studied (32 mild TBI and 32 moderate-severe TBI). Both groups were young (36.2 years mild TBI and 36.5 years moderate-severe TBI) and mostly male (69\% mild TBI and $84 \%$ moderate severe TBI). Early SD was present in $7(22 \%)$ moderate-severe TBI patients and $0(0 \%)$ mild TBI patients $(p<0.01)$. On multivariable regression, moderate-severe TBI was associated with an absolute $4.2 \%$ reduced FS compared to mild TBI $(95 \% \mathrm{Cl} 0.8 \%-7.6 \%, \mathrm{p}=0.02)$. All patients with early SD recovered to normal systolic function by 7-9 days injury (Figure 1).

\section{Conclusions:}

SD is common early after moderate-severe TBI and appears reversible over the first week of hospitalization. TBI severity is independently associated with worse systolic function. Early echocardiography is a safe, applicable, and feasible procedure following TBI and may aid clinicians with hemodynamic management post TBI. 
Poster 20

\title{
Detecting the Frequency and Severity of Paroxysmal Sympathetic Hyperactivity Symptoms in Acute Traumatic Brain Injury
}

\author{
Sophie Samuel.
}

Department of Pharmacy. Memorial Hermann Medical Center, Houston, TX, USA.

\section{Introduction:}

Paroxysmal sympathetic hyperactivity (PSH) is a neurological condition that occurs most frequently after traumatic brain injury (TBI). Sympathetic hyperactivity can manifest as increased heart rate, blood pressure, respiratory rate, temperature, sweating and posturing activity. In 2014 , a consensus statement proposed a diagnostic framework to identify PSH-like features using two assessment tools for severity and probability of diagnosis. The objective of this study is to report the incidence, frequency and severity of PSH symptoms in the acute setting after TBI using the new diagnostic criteria.

\section{Methods:}

This was a retrospective study from July 2014-August 2015. Included were all adult patients admitted to a tertiary care intensive care unit with a primary diagnosis of TBI and a length of stay longer than 14 days. Clinical endpoints examined were: tachycardia ( $H R \geq 100$ ), hypertension (SBP $\geq 160)$, fever (Temp $\geq 100.4 C$ ), tachypnea ( $R R \geq 24)$, and episodes of posturing. In addition, medications used to treat symptoms were recorded.

\section{Results:}

The most frequent symptom was tachycardia (53\%), followed by tachypnea (47\%), hypertension ( $26 \%)$, fever $(19 \%)$ and posturing (16\%). Symptoms occurred $\geq 2$ times daily in $64 \%$ of patients. $49 \%$ had at least 2 symptoms occurring simultaneously and $68 \%$ had symptoms persisting for 2 weeks. Using the diagnostic criteria, $74 \%$ of patients were categorized as possible or probable $\mathrm{PSH}(\mathrm{PSH}+)$ on day 14. Patients with PSH+ had lower GCS on admission; [3(3-6) vs $8(3-12) ; p=0.02]$ and at day $14 ;[10(7-14)$ vs $14(14-15) ; p<0.01$.] Medications often used to control symptoms included, anti-pyretics, opioids, betablockers, and alpha-2 agonist. There was a longer LOS in the PSH+ group [24(19-38) vs 17(14-26); $\mathrm{p}=0.03]$, but no difference in mortality.

\section{Conclusions:}

Symptoms of sympathetic hyperactivity were seen commonly after TBI in the acute setting. Using a recently proposed diagnostic framework, $\mathrm{PSH}$ likelihood was possible or probable in $74 \%$ of patient admitted with a hospital length of stay greater than 14 days. 


\title{
Poster 21
}

\section{Hypertonic Saline Is Superior To Mannitol In The Combined Effects On Intracranial Pressure And Cerebral Perfusion Pressure In Patients With Severe Traumatic Brain Injury}

\author{
Halinder S. Mangat ${ }^{1}$, Xian X. Wu ${ }^{2}$, Linda L. Gerber ${ }^{2}$, Justin J. Schwarz ${ }^{3}$, Malik M. Fakhar ${ }^{4}$, Santosh S. \\ Murthy ${ }^{4}$, Philip P. Stieg ${ }^{5}$, Jamshid J. Ghajar ${ }^{6}$, Roger R. Hart ${ }^{5}$.
}

\footnotetext{
${ }^{1}$ Weill Cornell Medicine / Neurology \& Neurosurgery, New York, NY, USA, ${ }^{2}$ Weill Cornell Medicine / Healthcare Policy and Research, New York, NY, USA, ${ }^{3}$ New York Presbyterian Hospital / Neurosurgery, New York, NY, USA, ${ }^{4}$ Weill Cornell Medicine / Neurology, New York, NY, USA, ${ }^{5}$ Weill Cornell Medicine / Neurosurgery, New York, NY, USA, ${ }^{6}$ Stanford University School of Medicine / Neurosurgery, Stanford, CA, USA.
}

\section{Introduction:}

Hypertonic saline (HTS) appears to be more effective than mannitol in reducing raised intracranial pressure (ICP) after severe traumatic brain injury (TBI). In this study we investigated which agent had superior combined effects on ICP and cerebral perfusion pressure (CPP).

\section{Methods:}

The Brain Trauma Foundation TBI-trac $®$ New York State database was used for this retrospective study. Patients who received only HTS were identified and matched with patients who received mannitol only (1:1 and 1:2 match). The two groups were matched for age, pupillary reactivity, Glasgow Coma Scale (GCS), CT abnormality, craniotomy and occurrence of hypotension on day 1 . Univariate analysis was performed to compare combined average and duration of ICP $>25 \mathrm{mmHg}$ (ICPhigh) and CPP $<60 \mathrm{mmHg}$ (CPPlow) (Wilcoxon signed rank test).

\section{Results:}

A total of 25 patients with severe TBI, who received only HTS were identified and matched with 25 (1:1) and 48 (1:2) patients who received mannitol only. In the 1:2 group 1 HTS patient was excluded, as there was no corresponding match in mannitol group. The mean age, GCS, incidence of abnormal pupils, hypotension, abnormal CT, craniotomy and day of ICP insertion were similar in the groups. There was no difference in number of days of ICP monitoring ( $p=0.09,0.46 ; 1: 1,1: 2$ groups). Osmolar doses were comparable; all patients in HTS group received 3\% HTS except one who received $23.4 \%$. In 1:1 match, number of days with CPPlow (2.0 \pm 1.7 vs. $3.6 \pm 2.8, p=0.03)$ was significantly lower in the HTS group. In addition, number of days with ICPhigh+CPPlow ( $0.6 \pm 0.8$ vs. $2.4 \pm 2.3, p=0.0007)$, averaged daily duration of ICPhigh+CPPlow ( $8.8 \pm 10.6$ vs. $28.1 \pm 26.9$ hours, $p=0.0004)$ and cumulative duration of ICPhigh+CPPlow (11.12 \pm 14.11 vs. $30.56 \pm 31.89$ hours, $p=0.01)$ were significantly lower in the HTS group. These results were reproduced in the 1:2 analyses.

\section{Conclusions:}

HTS is superior in its combined effect on ICP and CPP after severe TBI when compared to mannitol. 


\title{
Poster 22
}

\section{Phase II Clinical Trial of Atorvastatin in Mild Traumatic Brain Injury}

\author{
Claudia Robertson ${ }^{1}$, James J. McCarthy ${ }^{2}$, Emmy E. Miller ${ }^{3}$, Harvey H. Levin ${ }^{4}$, Stephen S. McCauley ${ }^{4}$, \\ Paul P. Swank.
}

${ }^{1}$ Baylor College of Medicine/Neurosurgery, Houston, TX, USA, ${ }^{2}$ University of Texas Health Sciences Center Houston/Emergency Medicine, Houston, TX, USA, ${ }^{3}$ Virginia Commonwealth

University/Neurosurgery, Richmond, VA, USA, ${ }^{4}$ Baylor College of Medicine/Physical Medicine and Rehabilitation, Houston, TX, USA, ${ }^{5}$ University of Texas Health Science Center at Houston/School of Public Health, Houston, TX, USA.

\section{Introduction:}

Statins constitute a class of medications commonly used in the treatment of elevated cholesterol. However, in experimental studies statins also have other non-cholesterol mediated mechanisms of action, which may have neuroprotective effects. The purpose of this study was to determine if administration of atorvastatin for 7 days after injury would improve neurological recovery in patients with mild traumatic brain injury $(\mathrm{mTBI})$.

\section{Methods:}

The hypothesis was that atorvastatin administration would reduce post-concussion symptoms and also that atorvastatin administration for one week post-injury would be safe. 140 patients with mTBI were planned to be enrolled and randomly assigned to receive atorvastatin $1 \mathrm{mg} / \mathrm{kg}$ (up to $80 \mathrm{mg} / \mathrm{kg}$ ) per day or placebo for 7 days starting within 24 hours of injury. Assessments of post-concussion syndrome, posttraumatic stress and depressive symptoms, cognition, memory, verbal fluency, functional, and work status were performed at baseline, 1 week, 1 and 3 months. The result on the Rivermead Post- Concussion Symptoms Questionnaire at 3 months was the primary outcome.

\section{Results:}

Enrollment in the trial was stopped early because of difficulty in recruiting sufficient numbers of subjects. 52 patients with $\mathrm{mTBI}$ were enrolled; 28 patients received atorvastatin and 24 received placebo. The mean Rivermead score was 7.6 for the atorvastatin group compared to 9.2 for the placebo group at 3 months post-injury $[F(1,4)=.1855, p=.6687)]$. The change in the Rivermead score between baseline and 3 months was also analyzed. The mean change in score was a decrease of 8.1 for the atorvastatin group and 10.7 for the placebo group $[F(1,47)=.4132, p=.5235]$. No serious adverse events occurred, and there was no significant difference in the incidence of adverse events in the two treatment groups.

\section{Conclusions:}

Atorvastatin administration for 7 days post-injury was safe, but there were no significant differences in neurological recovery after mTBI with atorvastatin. 


\section{Poster 23}

Association between comorbidities, nutritional status, and anticlotting drugs and neurological outcomes in geriatric patients with traumatic brain injury

Tomoya Okazaki, Toru T. Hihumi, Kenya K. Kawakita, Arisa A. Manabe, Hideyuki H. Hamaya, Natsuyo N. Shinohara, Hajime H. Shishido, Koshiro K. Takano, Yuko Y. Abe, Yasuhiro Y. Kuroda.

Emergency Medical Center, Kagawa University Hospital, Miki, Japan.

${ }^{* * * *}$ Permission was not granted to print this abstract ${ }^{* * * *}$ 


\title{
Poster 24
}

\section{Serum Cytokine Levels Help Predict Critical Increases In Intracranial Pressure}

\author{
Anthony Tannous ${ }^{1}$, Hegang H. Chen ${ }^{2}$, Peter P. Hu ${ }^{3}$, Ifeatu I. Onyekwelu ${ }^{4}$, Nehu N. Parimi ${ }^{4}$, Leslie L. Sult ${ }^{4}$,
} Shiming S. Yang ${ }^{3}$, Thomas T. Scalea ${ }^{5}$, Deborah D. Stein ${ }^{5}$.

\begin{abstract}
${ }^{1}$ University of Maryland Medical Center/ Department of Surgical Critical Care, Baltimore, MD, USA, ${ }^{2}$ University of Maryland Medical Center/Department of epidemiology, Baltimore, MD, USA, ${ }^{3}$ University of Maryland Medical Center / Department of Anesthesiology, Baltimore, MD, USA, ${ }^{4}$ University of Maryland Medical Center/ Center for Trauma and Anesthesiology research, Baltimore, MD, USA, ${ }^{5}$ University of Maryland Medical Center/ Department of Trauma Surgery, Baltimore, MD, USA.
\end{abstract}

\section{Introduction:}

An essential part of the management of traumatic brain injury (TBI) is the mitigation of secondary insults to the brain such as sustained increases in intracranial pressure (ICP). It would be beneficial to be able to predict increased ICP so as to facilitate safe transport of patients. Given the role of neuro-inflammation in secondary brain injury, we aimed to study the role of selected serum cytokines as predictors of critical increases in ICP.

\section{Methods:}

This is a prospective observational study of adult patients (age $\geq 18$ years) admitted to a level 1 trauma center with severe TBI between 11/13-8/15. Blood samples were obtained at 6 hour intervals for 3 days after admission. Receiver Operating Characteristic (ROC) curves were used to compare the predictive capacity of models using serum cytokine levels, vital signs, or a combination of the previous to predict elevation of ICP above 20 or $30 \mathrm{mmHg}$ for $5 \mathrm{~min}$ or more in the following 6 hours.

\section{Results:}

250 serum samples from 24 patients were matched to subsequent 6 hour periods of monitoring.. NI-VS and cytokine values had similar predictive ability $(0.77$ and 0.78$)$. There was a significant improvement in the predictive capacity of a combined model of NI-VS and IL4 level over NI-VS alone in predicting ICP elevation to $>20 \mathrm{mmHg}(0.8$ vs $0.77, \mathrm{p} 30 \mathrm{mmHg}(0.91$ vs $0.79 \mathrm{p}<0.005)$.

\section{Conclusions:}

Serum cytokine level measurement in TBI can help better predict sustained increases in ICP to critical levels when combined with physiological data. Even without invasive monitoring, predictions about impending elevations in ICP are possible and achieve good accuracy with the addition of cytokine measurements. 


\section{Poster 25}

\section{Assessment of Autonomic Nervous System Dysfunction to Predict Neuroworsening After Isolated Head Injury}

Christopher Melinosky ${ }^{1}$, Shiming S. Yang ${ }^{2}$, Peter P. Hu' ${ }^{2}$, Hsiao-Chi H. Li ${ }^{3}$, Imad I. Khan ${ }^{1}$, Colin C. Mackenzie ${ }^{2}$, Wan-Tsu W. Chang ${ }^{1}$, Gunjan G. Parikh ${ }^{1}$, Deborah D. Stein ${ }^{3}$, Neeraj N. Badjatia ${ }^{1}$.

${ }^{1}$ Neurocritical Care, Program in Trauma, Univ. of Maryland School of Medicine, Baltimore, MD, USA, ${ }^{2}$ Department of Anesthesiology, University of Maryland School of Medicine, Baltimore, MD, USA, ${ }^{3} R$ Adams Cowley Shock Trauma Center, University of Maryland School of Medicine, Baltimore, MD, USA.

\section{Introduction:}

Neuroworsening (NW) after traumatic brain injury (TBI) is a major cause of added morbidity, however, there is no reliable way to predict NW. We hypothesized that autonomic nervous system dysfunction (ANS) measured by analysis of plethysmograph variability (PPGV) in the first hour after presentation may predict NW in the initial 48 hours after TBI.

\section{Methods:}

Inclusion criteria included: age $\geq 18$, survival $>15$ minutes after arrival to the Trauma Resuscitation Unit, and head Abbreviated Injury Score(AIS)>1. Patients with systemic trauma were excluded. NW was defined as any of the following occurring in the first 48 hours: new asymmetric pupillary dilatation ( $>2 \mathrm{~mm}$ ), 2 point GCS decline, interval worsening of CT scan as assessed by the Marshall score, or requirement for neurosurgical intervention. The beat-to-beat variation of the PPG, and PPG morphologic features were calculated to quantify the ANS impact on the physiological status. Multivariate stepwise logistic regression was used to develop predictive models of NW.

\section{Results:}

There were 205 patients (mean age 43 years old, GCS 13, ISS 12, 27\% women) who met criteria between December 2011 and May 2013. NW occurred in 24(12\%) patients. PPGV analysis at 15 (PPG15) and 60(PPG60) minutes post-admission demonstrated predictive capability for $\mathrm{NW}(\mathrm{P}<0.01)$. PPGV15 was able to better discriminate NW as compared to a baseline model of age, sex, initial VS (ROC 0.91 v. $0.78, P=0.01$ ). PPGV60 better discriminated future NW as compared to the model of age, sex, admission VS and GCS (ROC $0.98 \vee 0.86, P=0.02$ ), and marginally better than a model combining admission VS, GCS, and Marshall score on CT(ROC 0.98 v 0.91,P=0.05).

\section{Conclusions:}

ANS dysfunction assessed by continuous PPG waveform analysis in the first hour represents a noninvasive early marker of future neurological deterioration after TBI. This technique may be coupled with clinical factors to more accurately predict NW, potentially leading to automated algorithms for earlier therapeutic interventions. 


\section{Poster 26}

\section{Management of Severe Traumatic Brain Injury At a Tertiary Health Center in Tanzania}

HALINDER S. MANGAT ${ }^{1}$, Luke L. Smart ${ }^{2}$, Benson B. Issarow ${ }^{3}$, Paul P. McClelland ${ }^{4}$, Gerald G. Mayaya ${ }^{5}$, Robert R. Peck ${ }^{6}$, Isidore I. Ngayomela ${ }^{7}$, Linda L. Gerber ${ }^{8}$, Xian X. Wu ${ }^{8}$, Philip P. Stieg ${ }^{9}$, Roger R. Hart ${ }^{9}$.

${ }^{1}$ Weill Cornell Medicine / Neurology \& Neurosurgery, New York, NY, USA, ${ }^{2}$ Weill Bugando School of Medicine; Weill Cornell Medicine / Global Health, Mwanza, Tanzania, United Republic of, ${ }^{3}$ Weill Bugando School of Medicine / Internal Medicine, Mwanza, Tanzania, United Republic of, ${ }^{4}$ Weill Cornell Medicine / Global Health, New York, NY, USA, ${ }^{5}$ Weill Bugando School of Medicine / Neurosurgery, Mwanza, Tanzania, United Republic of, ${ }^{6}$ Weill Cornell Medicine / Global Health, Mwanza, Tanzania, United Republic of, ${ }^{7}$ Weill Bugando School of Medicine / Orthopedics, Mwanza, Tanzania, United Republic of, ${ }^{8}$ Weill Cornell Medicine / Healthcare Policy and Research, New York, NY, USA, ${ }^{9}$ Weill Cornell Medicine / Neurosurgery, New York, NY, USA.

\section{Introduction:}

Severe traumatic brain injury (TBI) is the number one cause of death and disability among young adults worldwide. Formulation and subsequent adherence to the Brain Trauma Foundation (BTF) Guidelines has been associated with reduced mortality after severe TBI. In this study we studied epidemiology and treatment of severe TBI at a tertiary referral hospital in Tanzania in reference to the BTF Guidelines.

\section{Methods:}

Patients with TBI hospitalized at Bugando Medical Centre, a Tanzanian tertiary referral hospital were recorded in a prospective registry. Demographics, cause of trauma, clinical characteristics, hospital care, and mortality were recorded for 14 days and on the day of discharge.

\section{Results:}

Between September 2013 and October 2015, of 371 patients with TBI 33\% (115/371) had severe TBI $(\mathrm{GCS}<9)$. Their mean age was 32.0 years, and the majority was male $(92 / 115,80.0 \%)$. Road traffic injuries were the most common cause of severe TBI $(75 / 115,65.2 \%)$. Only half of the patients $(55 / 115$, $47.8 \%)$ were hospitalized on the same day as their injury. CT imaging was performed in $49.6 \%(57 / 115)$, and $58.3 \%(67 / 115)$ were admitted to the ICU. Continuous blood pressure monitoring and intracranial pressure (ICP) monitoring were not performed for any patient. Thirty-eight percent (44/115) received hyperosmolar therapy and $35.7 \%$ (41/115) underwent craniotomy. Two-week mortality was $34.8 \%$ (40/115).

\section{Conclusions:}

Pre-hospital and routine ICU care, CT imaging, blood pressure and ICP monitoring are underutilized or unavailable in the management of severe TBI in the tertiary referral hospital setting. TBI associated mortality is significantly higher than that in high-income countries. Improving outcomes after severe TBI will require concerted investment in pre-hospital care as well as improvement in availability of neuroimaging, ICU resources and expertise in multidisciplinary care. Establishment of comprehensive trauma registries is key to elucidate such data. 


\section{Poster 27}

\section{Bedside Stratification of Intracranial Pressure Elevations after Severe Traumatic Brain Injury}

Brandon Foreman, $M D^{1}$, Brittany B. Staarmann, $M D^{2}$, Christopher C. Zammit, $M D^{3}$, Jason J. Hinzman, $P h D^{2}$, Jed J. Hartings, $P h D^{2}$, Opeolu O. Adeoye, $M D^{3}$, Norberto N. Andaluz, MD'.

${ }^{1}$ University of Cincinnati, Department of Neurology \& Rehabilitation Medicine, Cincinnati, OH, USA, ${ }^{2}$ University of Cincinnati, Department of Neurosurgery, Cincinnati, OH, USA, ${ }^{3}$ University of Cincinnati, Department of Emergency Medicine, Cincinnati, OH, USA.

\section{Introduction:}

Elevated intracranial pressure (ICP) is thought to mediate secondary brain injury by decreasing cerebral perfusion pressure (CPP) and reducing cerebral blood flow. Clinical trials targeting ICP thresholds have not demonstrated benefit. We hypothesized that stratifying elevations in ICP based on their effect on CPP would be feasible with the use of continuous, time-resolute neuromonitoring data.

\section{Methods:}

We studied a convenience sample of five patients undergoing intracranial neuromonitoring after severe traumatic brain injury per our institutional protocol. Patients had a parenchymal ICP monitor time-synced with continuous arterial blood pressure. Waveform data were recorded into Moberg CNS monitors, and second-by-second averages were exported into LabChart for quantification. Elevations in ICP $\geq 20 \mathrm{mmHg}$ were identified and their duration was measured along with CPP. Elevations were stratified into those that exhibited a reciprocal decrease in CPP (ICP[neg]) and those with stable or elevated CPP (ICP [preserved]).

\section{Results:}

The mean patient age was 33; three were male. A total of 290 individual elevations in intracranial pressure were observed over a monitoring duration of 31,573 minutes. Of those, $172(59 \%)$ had stable or elevated CPP (ICP[preserved]; $\triangle$ CPP 5.7+/-14.8) while 118 (41\%) exhibited decreased CPP (ICP [neg]; $\triangle$ CPP -16.7+/-11.2; $p<0.01)$; the maximum ICP in each group was similar (35.8+/-14.6 vs. 36.6+/- 15.3; $p=0.69)$. ICP[neg] were briefer compared to ICP[preserved] (863+/-605s vs. $1158+/-1124 ; p<0.01)$. $90 / 118(76 \%)$ of ICP[neg] were observed in patients who went on to have poor outcome (death or dependency).

\section{Conclusions:}

We found ICP elevations that reciprocally decreased CPP (ICPneg) were identifiable using bedside neuromonitoring devices, and that these elevations resulted in a CPP drop of nearly $17 \mathrm{mmHg}$, despite a similar maximum ICP despite a shorter duration. The majority of these ICP elevations occurred in patients with poor outcome, and may represent a target for aggressive ICP lowering therapy. 


\title{
Poster 28
}

\section{Routine Repeat CT Head Is Not Necessary Without Neurological Change For Mild Traumatic Brain Injury}

\author{
Andrew Montoure, Ha H. Nguyen, Ashish A. Sharma, Vijay V. Johnson.
}

Medical College of Wisconsin, Milwaukee, WI, USA.

\section{Introduction:}

Mild traumatic brain injury (mTBI) is defined as an initial Glasgow Coma Scale (GCS) 13-15. Current recommendations include a follow-up computed tomography (CT) scan of the head prior to discharge. Often, imaging and neurological exam remains stable, questioning the role of routine repeat imaging.

\section{Methods:}

A retrospective chart review was completed on TBI patients evaluated at a level 1 trauma center between August 2009 and December 2015. Inclusion criteria included: initial GCS 13-15, blunt head injury, and available repeat imaging. Exclusion criteria included GCS $<13$, penetrating trauma, those that required immediate surgery, or those without repeat imaging. A total of 198 patients were included in the analysis. Statistics were done with Mann-U Whitney or Chi-Square testing.

\section{Results:}

Age was $49.3 \pm 21$ years. There were 123 males and 75 females. The most common mechanism was falls (43.9\%), followed by motor-vehicle collision (22.7\%), motor-cycle accident (12.6\%), assault $(9.1 \%)$, pedestrian-struck by automobile (6.1\%), and bike-related (5.6\%). Polytrauma occurred in $42.6 \%$, anticoagulation / antiplatelet $(\mathrm{A} / \mathrm{A})$ use in $22.2 \%$, and skull fractures in $50.5 \%$. On repeat $\mathrm{CT}, 16.2 \%$ were better, $59.1 \%$ were stable, $24.7 \%$ were worse. Only 19 patients $(19.6 \%)$ exhibited neuro-exam changes, where 16 patients received repeat imaging which ultimately demonstrated stable findings. Ultimately, only one patient required a neurosurgical procedure, an external ventricular drain, due to significant decline. Age, gender, mechanism, polytrauma, A/A use, and skull fractures did not significantly correlate with neuro-exam changes. On the other hand, neuro-exam changes significantly correlated with CT changes $(p=0.01)$.

\section{Conclusions:}

Repeat imaging tend to show worse findings when associated with neurological changes. On the other hand, results for repeat imaging were variable without neurological changes and generally did not alter treatment plans / patient outcomes. As CT imaging inflicts radiation exposure and hospital costs, repeat imaging is only warranted for neurological changes that may necessitate a neurosurgical procedure. 


\title{
Poster 29
}

\section{Contrasting Preferences on the Communication of Prognosis between Family Members and Physicians during Goals-of-Care Decisions in Critically-III TBI Patients - Results from a Multi- Center Qualitative Study}

\author{
Thomas Quinn ${ }^{1}$, Jesse J. Moskowitz ${ }^{2}$, Lori L. Shutter ${ }^{3}$, Rob R. Goldberg ${ }^{4}$, Nananda N. Col ${ }^{5}$, Kathy K. \\ Mazor ${ }^{6}$, Susanne S. Muehlschlegel'.
}

\begin{abstract}
${ }^{1}$ University of Massachusetts, Worcester, MA, USA, ${ }^{2}$ University of Massachusetts Medical School, Worcester, MA, USA, ${ }^{3}$ University of Pittsburgh Medical Center / Dept. of Neurology and Critical Care, Pittsburgh, PA, USA, ${ }^{4}$ University of Massachusetts Medical School / Dept. of Quantitative Health Sciences, Worcester, MA, USA, ${ }^{5}$ University of New England / Dept. of Internal Medicine, Biddeford, ME , USA, ${ }^{6}$ University of Massachusetts Medical School / Dept. of Primary Care, Worcester, MA, USA, ${ }^{7}$ University of Massachusetts Medical School / Dept. of Neurology, Worcester, MA, USA.
\end{abstract}

\section{Introduction:}

Currently, research is underway to create a shared decision-making (SDM) tool for goals-of-care decisions in critically-ill traumatic brain injury (ciTBI) patients. SDM is a collaborative process aimed at standardizing prognostication, enhancing family members' understanding about prognosis, actively weighing risks and benefits of a treatment, and matching them to patient values and preferences. To create a meaningful tool, we explored family members' and physicians' perspectives on prognosis communication during goals-of-care discussions for ciTBI patients employing mixed-methods.

\section{Methods:}

We conducted semi-structured interviews with 16 ciTBI surrogate decision-makers from two level-1 trauma centers, and 20 attending physicians representing geographic (Northeast,MidAtlantic,South,West,Midwest] and subspecialty diversity (neurocritical care,neurosurgery,trauma, palliative care). Two independent reviewers analyzed transcribed interviews using deductive and inductive approaches (NVIVO-software). The sample size was determined by theme saturation.

\section{Results:}

We found strikingly contrasting opinions between physicians and surrogates regarding use of numbers/percentages during prognostication. Overall, $82 \%$ of surrogates stated a preference for prognosticated outcomes expressed as percentages, and 100\% preferred prognosis in a "more direct" manner. Surrogates favored percentages because they were "more clear, more concise, and less confusing". In contrast, $75 \%$ of physicians stated that they do not use precise percentages when discussing prognosis in ciTBI due to distrust in the predictive accuracy of existing data: "Better have damn good data to do that with, and most often, we do not." Physicians also voiced concern over families' interpretation of percentages: "Families will often hook onto those numbers and make an absolute judgment...they become simplified and [these numbers are] used against you later."

\section{Conclusions:}

The dissimilar preferences for the use of percentages and numbers during prognostication represent an important difference between surrogates and physicians. These findings have a direct impact on the design of a goals-of-care SDM tool for ciTBI. A future goals-of-care decision aid will require iterative physician and surrogate feedback and feasibility testing in the ICU. 
Poster 30

\title{
IMPACT OF EMERGENCY DECOMPRESSIVE CRANIECTOMY FOLLOWING BLUNT TRAUMATIC BRAIN INJURY ON MORTALITY: PROPENSITY MATCHED ANALYSIS
}

\author{
Nasim Ahmed.
}

Jersey Shore University Medical Center, Division of Trauma \& Neuro Critical Care, Neptune, NJ, USA.

\section{Introduction:}

Decompressive craniectomy (DC) is not a new procedure, however, it has gained momentum in recent years, in the management of refractory intracranial hypertension. However, the timing of DC has not been well studied and a lack of evidenced-based guidelines for the optimal timing of DC has resulted in a wide variability in practice patterns. In most instances, DC has been performed based on neurosurgical evaluation of the patient with or without intracranial pressure (ICP) monitoring and evidence of increased ICP and /or evidence of edema on imaging studies of the brain at the point of care. Previous studies have shown variable outcomes in emergency (within 4 hours of injury) neurosurgical procedure in Traumatic Brain Injury (TBI). The purpose of this study was to evaluate the impact of emergency DC on in-hospital mortality following blunt TBI.

\section{Methods:}

Data was retrieved from the National Trauma Data Bank (NTDB) data set (RDS_2007-RDS_2010). All craniectomies performed on patients with a blunt mechanism of head injury within twenty-four hours of admission, were included in the study. In- hospital mortality was the main outcome of interest.

\section{Results:}

942 patients qualified for the study. 669 patients $(71 \%)$ underwent a craniectomy within 4 hours (emergency group)and 273 patients (29\%) had craniectomy performed between $>4$ hours hours to 24 hours following hospital arrival (late group). Propensity matched analysis identified 268 pairs of patients in both groups. The mean standardized differences were less than $10 \%$ after matching. There were no significant differences in mortality [odd ratio $1.018, \mathrm{Cl}(0.689,1.506), \mathrm{P}=1.00$ ], Absolute Risk Reduction $[0.004,95 \% \mathrm{Cl}(-0.078,0.085), \mathrm{P}=1.00]$ and length of stay between the groups [hazard ratio, $0.770,95 \%$ $\mathrm{Cl}(0.56,1.059], \mathrm{P}=0.108]$.

\section{Conclusions:}

No difference was seen on in-hospital mortality between patients operated within 4 hours versus patients operated between 4 and 24 hours of admission. 


\title{
Poster 31
}

\section{Bedside Detection of Spreading Depolarizations using Cortical Depth Electrodes in Severe Non- Surgical Traumatic Brain Injury: Preliminary Experience.}

\author{
Brandon Foreman, $M D^{1}$, Jason J. Hinzman, $P h D^{2}$, John J. York, $M D^{2}$, Ryan R. Tackla, $M D^{2}$, Bryan B. \\ Krueger, $M D^{2}$, Daniel D. Kanter, $M D^{1}$, Norberto N. Andaluz, $M D^{2}$, Jed J. Hartings, $P h D^{2}$.
}

${ }^{1}$ University of Cincinnati, Department of Neurology \& Rehabilitation Medicine, Cincinnati, OH, USA, ${ }^{2}$ University of Cincinnati, Department of Neurosurgery, Cincinnati, OH, USA.

\section{Introduction:}

Spreading depolarizations (SDs) are pathological waves of neuronal depolarization that occur in $56 \%$ of patients with traumatic brain injury (TBI) who require surgical treatment of focal lesions. The incidence of SDs in non-surgical TBI is unknown, but in theory SDs can be detected using cortical depth electrodes (dEEG) placed at bedside via burrhole. We hypothesized that the incidence of SDs recorded using dEEG in non-surgical patients would be similar to that documented in surgical TBI.

\section{Methods:}

For the study period starting 4/2015, we included severe, non-penetrating TBI who did not require urgent neurosurgery on admission. All patients underwent bedside burrhole placement of intracranial pressure, tissue oxygen, regional blood flow and dEEG monitors via a single quad-lumen bolt per institutional protocol. Data were recorded on Moberg CNS with DC-coupled amplifiers.

\section{Results:}

Over a 12-month period, 15 patients (age 44+/-19; 80\% male; GCS range 3-7) underwent monitoring for a mean duration of $97.7+/-43.5$ hours, beginning 11.0 hours (median, quartiles: 8.1-18.9) after injury. 12/15 $(80 \%)$ monitoring devices were placed in non-dominant frontal lobe, targeting cerebral cortex. $5 / 15$ patients had focal pathology in the monitored lobe, while 4/15 had contralateral frontal lesions and 6/15 had diffuse injury or involvement of other lobes. Overall, $2 / 15(13 \%)$ exhibited SDs. $7 / 15(47 \%)$ patients died, including those with SDs. There were no significant hematomas or infections related to invasive neuromonitoring.

\section{Conclusions:}

The incidence of SDs detected with dEEG placed in non-dominant frontal lobe was lower than previously reported with injury-targeted placement of subdural strips in surgical TBI patients. This may be due to more restricted spatial sampling of dEEG, lack of targeting to injured peri-lesional tissue, and/or lower innate incidence of SD in patients with non-surgical or more diffuse injuries. Targeted placement of subdural electrodes through burr holes may be warranted in non-surgical TBI patients. 


\title{
Poster 32
}

\section{IMPACT Traumatic Brain Injury Prognostic Calculations Use By Clinicians: A Multidisciplinary, International Survey}

\author{
Casey Rommel ${ }^{1}$, Gregory G. Hawryluk ${ }^{2}$, Joshua J. Letsinger ${ }^{3}$, Ryan R. Hirshi ${ }^{3}$, Raminder R. Nirula ${ }^{4}$ \\ ${ }^{1}$ University of Utah Biomedical Informatics, Salt Lake City, UT, USA, ${ }^{2}$ University of Utah Neurosurgery, \\ Salt Lake City, UT, USA, ${ }^{3}$ University of Utah Neurosurgery, Salt Lake City, UT, USA, ${ }^{4}$ University of Utah \\ General Surgery, Salt Lake City, UT, USA.
}

\section{Introduction:}

Traumatic brain injury (TBI) is a prevalent condition that is responsible for a significant amount of disability and healthcare expenditures. Clinicians can and do use the IMPACT prognostic calculations to inform care decisions. This study was undertaken to examine self-reported individual and institutional use of the IMPACT prognostic calculations in an effort to identify trends and effects on treatment.

\section{Methods:}

We conducted an international and multidisciplinary survey examining self-reported awareness and use of the IMPACT prognostic calculator. Factors associated with awareness and use of the calculator including provider specialty, years in practice, personal and institutional volume of TBI patients treated, and institutional trauma level were also examined. Members of the AAST were provided web link to the voluntary and anonymous survey in an email. Study data was collected and managed using REDCap. STATA/IC 13 Statistical Software was used for analysis.

\section{Results:}

76 respondents $(49.7 \%)$ were aware of the calculator, only $5.3 \%$ (4) said that they often, and $48.7 \%$ (37) said they sometimes used it. Volume of TBI cases and specialty both were positively associated with awareness and use of the calculator. 42 providers often or sometimes used calculator, $88.1 \%$ (37) stated that it had some influence on their care for the patients. $88.1 \%$ used the information to better communicate with the patient's family. $26.2 \%$ used the calculator to proceed more quickly to a lower level of care and $19 \%$ used it to provide more aggressive care.

\section{Conclusions:}

Of those aware, still only slightly more than half (54\%) used it. The use of the IMPACT calculator was mainly to better communicate with patient family, but a portion, $26.2 \%$ and $19 \%$ of providers, said it influenced their care in other ways. These results provide direction to increase awareness and use of the IMPACT prognostic calculations. 


\title{
Poster 33
}

\section{Intensivist Practices in the Management of Severe Traumatic Brain Injury: A Hemodynamic and Neuromonitoring Survey}

\author{
Sanjeev Sivakumar ${ }^{1}$, Mohammed M. Rehman ${ }^{2}$, Holly H. Hinson ${ }^{3}$, Neeraj N. Naval ${ }^{4}$, Christos C. \\ Lazaridis'.
}

\begin{abstract}
${ }^{1}$ Baylor College of Medicine/ Department of Neurology/Neurocritical Care, Houston, TX, USA, ${ }^{2}$ Henry Ford Hospital/ Department of Neurology/Neurocritical Care, Detroit, MI, USA, ${ }^{3}$ Oregon Health Sciences University/ Department of Neurology/Neurocritical Care, Portland, OR, USA, ${ }^{4}$ Baptist Medical Center, Neurocritical Care, Jacksonville, FL, USA.
\end{abstract}

\section{Introduction:}

The Brain Trauma Foundation guidelines suggest individualizing cerebral perfusion pressure (CPP) goals based on tissue oxygenation (PbtO2), pressure reactivity (index; PRx), and metabolism (lactate- pyruvate ratio; LPR). Our objectives were to investigate practices pertaining to bedside hemodynamic and neuromonitoring in TBI patients, and to analyze differences among "neurointensivists" (NIs; defined as clinical practice comprised $>1 / 3$ by neurocritical care) and other intensivists (Ols).

\section{Methods:}

Electronic survey of 22 items including a TBI case-scenario; endorsed by SCCM (9,000 recipients) and ESICM (on-line newsletter) in 2013. Chi-square test was used to compare proportions of responses between NIs and Ols with a significance $p<0.05$.

\section{Results:}

There were 655 responders (66\% completion rate); $422(65 \%)$ were classified as Ols and $226(35 \%)$ as NIs. Use of neuromonitoring-derived variables to optimize CPP in patients with severe TBI, for the entire cohort: PbtO2 (18\%), transcranial Doppler(TCD) (12\%), jugular venous bulb (11\%), CT Perfusion(CTP) (4\%), PRx (4\%), and LPR (3\%). NIs use more PbtO2 (28\% vs. $10 \%, p=0.0001)$ and CTP $(7.6 \%$ vs. $2.5 \%$, $\mathrm{p}=0.012)$. More NIs have a hemodynamic protocol $(44.5 \%$ vs. $33.3 \%, p=0.007)$ for TBI, use more arterial waveform analysis $(45 \%$ vs. $35 \%, p=0.019)$, and bedside ultrasound $(37 \%$ vs. $27.7 \%, p=0.023)$, while more Ols monitor mixed venous oxygen saturation $(54.1 \%$ vs. $45 \%, p=0.045)$. In the case scenario of raised ICP, low PbtO2, and preserved pressure autoregulation, most responders employ analgesia and/or sedation (47\%) and osmotherapy (38\%); fewer make use of preserved pressure reactivity $(28 \%)$, (vasopressor use $34.5 \%$ NIs vs. $23 \%$ Ols, $p=0.014$ ).

\section{Conclusions:}

"Neurointensivists" employ more hemodynamic and neuromonitoring to patients with TBI. Intracranial pressure and CPP remain cornerstones of management, however the use of other physiologic variables like PbtO2, PRx, and LPR, is infrequent. Intensivists make limited efforts to target patient-specific ICP/CPP goals. 


\title{
Poster 34
}

\section{Predicting Survival after Acute Civilian Penetrating Brain Injuries: The SPIN Score}

\author{
Susanne Muehlschlegel', Didem D. Ayturk', Aditi A. Ahlawat ${ }^{3}$, Saef S. Izzy ${ }^{4}$, Thomas T. Scalea ${ }^{5}$, Deborah
} D. Stein ${ }^{5}$, Timothy T. Emhoff', Kevin K. Sheth ${ }^{6}$.

\begin{abstract}
${ }^{1}$ Univ of Massachusetts Med School, Dept. Neurology, Anesthesia/Crit Care \& Surgery, Worcester, MA, USA, ${ }^{2}$ University of Massachusetts Medical School, Dept. Surgery, Worcester, MA, USA, ${ }^{3}$ University of Massachusetts Medical School, Dept. Neurology, Worcester, MA, USA, ${ }^{4}$ Massachusetts General Hospital and Brigham \& Women's Hospital, Harvard Medical School, Dept. of Neurology (Division of Neurocritical

Care), Boston, MA, USA, ${ }^{5}$ R Adams Cowley Shock Trauma Center, University of Maryland, Dept. of Surgery, Baltimore, MD, USA, ${ }^{6}$ Depts. of Neurology (Neurocritical Care and Emergency Neurology) and Neurosurgery, Yale University, New Haven, CT, USA.
\end{abstract}

\section{Introduction:}

The predominant experience of penetrating traumatic brain injury ( $\mathrm{pTBI}$ ) derives from battlefield settings, but the civilian experience in Western settings in patients treated after 2005 is limited to only small and single-center studies. As a result, outcome predictors of civilian pTBI in modern trauma and neurocritical care settings are poorly defined. The aim of this study was to identify predictors associated with survival in a contemporary, large, diverse two-center pTBI cohort, and to develop a parsimonious survival prediction score for civilian pTBI.

\section{Methods:}

Our cohort comprised 415 pTBI patients retrospectively identified from the local trauma registries at two U.S. level-1 trauma centers, of which one was predominantly urban and the other predominantly rural. Predictors of in-hospital and 6-month survival identified in univariate and multivariable logistic regression were used to develop the simple Surviving Penetrating Injury to the BraiN (SPIN) Score.

\section{Results:}

The mean age was $33 \pm 16$ years, and patients were predominantly male $(87 \%)$ and black $(58 \%)$. Survival at hospital discharge and 6-months post pTBI was $42.4 \%$. Motor Glasgow Coma sub-score, pupillary reactivity, self-inflicted injury, transfer from other hospital, female sex, Injury Severity Score and INR were independently associated with survival (all $p<0.001$; area-under-the-curve 0.962). Important radiological factors associated with survival were also identified but their addition to the full multivariable would have resulted in model overfitting without much gain in the area-under-the-curve.

\section{Conclusions:}

We developed the SPIN Score, a logistic regression-based risk stratification scale estimating survival after pTBI. While external validation is warranted, this clinical survival prediction tool may provide important information to guide families and physicians during intervention- and goals-of-care decisionmaking after civilian pTBI. 


\title{
Poster 35
}

\section{Real-time visualization of the cumulative pressure and time dose of intracranial pressure in individual traumatic brain injured patients.}

\author{
Geert Meyfroidt ${ }^{1}$, Marine M. Flechet ${ }^{2}$, Bart B. Depreitere ${ }^{3}$, Greet G. Van den Berghe ${ }^{1}$, Fabian F. Güiza \\ Grandas ${ }^{4}$.
}

${ }^{1}$ Department of Intensive Care Medicine, University Hospitals Leuven, Leuven, Belgium, ${ }^{2}$ Laboratory of Intensive Care Medicine, KU Leuven, Leuven, Belgium, ${ }^{3}$ Department of neurosurgery, University Hospitals Leuven, Leuven, Belgium, ${ }^{4}$ Laboratory of Intensive Care Medicine, KULeuven, Leuven, Belgium.

\section{Introduction:}

The 'dose' of intracranial hypertension, a summary measure of duration and intensity of elevated intracranial pressure (ICP) episodes, is associated with worse outcome in traumatic brain injury (TBI) [12]. Although this cumulative ICP dose is easy to calculate in retrospect, no clinical monitor exists that presents the burden of ICP in real time to the bedside. [1] Vik et al. J Neurosurg 109:678-684 [2] Güiza et al. Intensive Care Med. 2015 Jun; 41(6):1067-76

\section{Methods:}

Retrospective analysis of minute-by-minute ICP monitoring data from a large multicenter database of TBI patients. A snapshot of the ICP dose experienced up to that moment is taken every minute, based on the population-based color-coded plots by Güiza et al, where 'bad' ICP episodes are red, and 'good' ICP episodes are blue. The ICP insult currently experienced by the patient, together with his ICP episodes of the previous 6 hours, and the cumulative ICP burden since ICU admission, are shown.

\section{Results:}

When playing these minute-by-minute snapshots consecutively, an animation is created showing the current and cumulative burden of ICP of the patient. We present the clinical course of 2 patients, 1 with good outcome (GOS 5), and 1 who died (GOS 1).

\section{Conclusions:}

Clinical trials aiming at aggressively treating ICP below a fixed threshold of $20 \mathrm{mmHg}$ have given disappointing results. Taking into account the cumulative pressure and time dose of ICP at different thresholds, could present a new way to define secondary injury by ICP, and a future target for therapy. The proposed method visualizes the current and cumulative time and pressure burden of ICP for individual patients, which could help a neuro-intensivist in identifying when a patient is currently in a state of potentially harmful elevated ICP, or when his outcome is at a turning point. 


\title{
Poster 36
}

\section{Acute Blood pressure variation and mortality in severe Traumatic Brain injury}

\author{
Chitra Sivasankar', Vasu V. Saini², Kristine K. O'Phelan ${ }^{3}$.
}

\footnotetext{
${ }^{1}$ University Of Miami/ Dept Of Neurology --Division Of Neuro critical acare, Miami, FL, USA, ${ }^{2}$ Dept Of Neurology, Miami, FL, USA, ${ }^{3}$ University Of Miami/Miller school of Medicine, Dept Of Neurology, Miami, FL, USA.
}

****Permission was not granted to print this abstract ${ }^{* * * *}$ 


\section{Poster 37}

\section{Relevance of Recurrence Rate and Air in Trepanation for Chronic Subdural Hematoma}

SHUN SUZUKI, Hiroyuki H. Nakajima, Hiroki H. Kurita.

International Medical Center, Saitama Medical University / Cerebrovascular Surgery, Hidaka, Japan.

\section{Introduction:}

Trepanation is standard of care for chronic subdural hematoma, while high recurrence rate is reported. Based on the data that air contamination during trepanation can relate to the recurrence, we paid close attention not to contaminate the air during the operation and evaluate after that. The purpose of this study is to determine the risk factors such as the amount of air contaminated using the data of our center since April 2011 to March 2015.

\section{Methods:}

We retrospectively evaluate 249 temperations (282hematomas) from April 2011 to March 2015 operated by one young neurosurgeon. During the operation, we made sure to put the burr hole the highest, not to break hematoma outer membrane and to put a drainage tube into the right position. We scaled the amount of hematoma by hand free ROI using head CT before operation and also the next day to evaluate the improvement rate.

\section{Results:}

The recurrence rate was $8.03 \%$ (20 cases). The average amount of air contaminated was $12.3 \mathrm{ml}$ and the improvement rate was $56.1 \%$, both of which didn't relate to the recurrence. No relevance among the recurrence rate, the amount of air contaminated and the hematoma improvement rate could be found.

\section{Conclusions:}

Though the amount of air contaminated during temperation doesn't relate to the recurrence rate, it was useful to scale the hematoma improvement rate and the amount of air in order to objectively assess the operation skill. With the experience, air contamination and recurrence became less, which suggest that we need to investigate the operation for chronic sundial hematoma further after we operate more. 


\title{
Poster 38
}

\section{Trans-cranial Doppler as an Inference Tool of Cerebral Hypoxic Episodes Early after Moderate and Severe TBI}

\author{
Catalina Sokoloff ${ }^{1}$, David D. Williamson ${ }^{2}$, Albert A. Martin ${ }^{3}$, Karim K. Serri ${ }^{3}$, Emmanuel E. Charbonney ${ }^{3}$, \\ Céline C. Odier ${ }^{4}$, Jean-François J. Giguère ${ }^{5}$, Francis F. Bernard ${ }^{3}$.
}

\begin{abstract}
${ }^{1}$ Hôpital Sacré-Coeur de Montréal, Emergency department, Université de Montréal, Montréal, Canada, ${ }^{2}$ Hôpital Sacré-Coeur de Montréal, Université de Montréal, Montréal, Canada, ${ }^{3}$ Hôpital Sacré-Coeur de Montréal, Critical Care, Université de Montréal, Montréal, Canada, ${ }^{4}$ Centre Hospitalier Universitaire de Montréal Université de Montréal, Montréal, Canada, ${ }^{5}$ Hôpital Sacré-Coeur de Montréal, neurosurgery Univertité de Montréal, Montréal, Canada.
\end{abstract}

\section{Introduction:}

The aim of this prospective observational study was to evaluate if trans-cranial Doppler (TCD) ultrasonography can be used as an inference tool of cerebral hypoxic episodes in patients with moderate to severe traumatic brain injury.

\section{Methods:}

Recruited patients had serial TCD studies to assess blood flow velocity of the middle cerebral artery (MCA). Measurements were done on bilateral MCAs as soon as logistically possible after the insertion of $\mathrm{PbtO} 2$ monitoring, once a day for a total of 5 days, and during dynamic challenge tests when feasible. Multiple physiologic parameters were registered concomitantly with each TCD measurement, with a particular focus on determinants of $\mathrm{PbtO} 2$ and potential confounding factors.

\section{Results:}

We studied 17 consecutive patients with a total of 85 TCD studies, of which $29(34 \%)$ were performed during an episode of cerebral hypoxia (PbtO2 $\leq 20 \mathrm{mmHg}$ ). PbtO2 monitoring was inserted 7 hours (IQR, 5-14.5h) after TBI. When considering all readings, we found no correlation between PbtO2 and MCA's mean blood flow velocity (Vmean). However, when looking only at episodes of cerebral hypoxia, a weak relationship could be established in readings collected $\leq 24 \mathrm{~h}$ from trauma $(r 2=0.41)$, but was lost for readings $>24 \mathrm{~h}$. For values collected $\leq 24 \mathrm{~h}$, all Vmean $<40 \mathrm{~m} / \mathrm{s}$ were associated with a PbtO2 $\leq 20 \mathrm{mmHg}$. These associations were independent of other factors usually linked to $\mathrm{PbtO} 2$, like $\mathrm{PaO} 2, \mathrm{PaCO} 2, \mathrm{Hb}$ level, ICP, and CPP.

\section{Conclusions:}

Measurements compatible with low cerebral blood flow velocity $(<40 \mathrm{~m} / \mathrm{s})$ collected in the first $24 \mathrm{~h}$ seem to correlate with brain tissue hypoxia and could be use as a screening tool to help minimise timesensitive secondary injury during that period. Otherwise, Vmean is not correlated to PbtO2. 


\title{
Poster 39
}

\section{Computerized Classification of Brain Pathophysiological Status after Severe Traumatic Brain Injury}

\author{
Nadia Savy ${ }^{1}$, Sina S. Fartoumi ${ }^{2}$, Francis F. Bernard ${ }^{3}$, Hussein H. Assaf ${ }^{4}$, Mohamad M. Sawan ${ }^{4}$, Guillaume \\ G. Emeriaud ${ }^{1}$.
}
${ }^{1} \mathrm{CHU}$ Sainte Justine - Pediatric Intensive Care Unit, Montréal, Canada, ${ }^{2}$ Ecole Polytechnique Montréal - Electrical Engineering Department, Montréal, Canada, ${ }^{3}$ Hôpital du Sacré-Coeur de Montréal - Neuro Intensive Care Unit, Montréal, Canada, ${ }^{4}$ Ecole Polytechnique de Montréal - Electrical Engineering Department, Montréal, Canada.

\section{Introduction:}

A precise assessment of brain condition after severe traumatic brain injury (TBI) is crucial to reduce secondary injuries and sequelae. Multimodal neuromonitoring permits to assess multiple systemic and brain parameters, but these data are complex to interpret continuously, especially in the overwhelmed environment of intensive care unit (ICU). Computerized Decision Support Systems (CDSS) can assist the clinicians in optimizing care. This study aims to evaluate an algorithm for classifying the cerebral condition, as a first step in the development of a CDSS.

\section{Methods:}

The study was approved by the local Research Ethics Committee. Patients with severe TBI (Glasgow coma score $<8$ ) with a monitoring of intracranial pressure and brain tissue oxygenation pressure were eligible. Data were extracted from the existing ICU electronical medical records (Semi Solutions Médicales). An incremental learning fuzzy min-max neural networks (FMMNs) classifier that adjusts its parameters online was implemented. The different cerebral status categories included: control condition, risk of ischemia or hyperemia, with/without intracranial hypertension. Previously validated and published datasets were used to train the system. The system was then tested with the patients' data and compared to a classification made by two clinical experts.

\section{Results:}

Eight 2-hour recording periods from adults with severe TBI were analyzed. The pathophysiological status was appropriately classified by the CDSS in (median) $93 \%$ (interquartile: $91-97 \%$ ) of time. Every critical event was detected, but brief misclassifications were frequently observed during the transition periods.

\section{Conclusions:}

In this preliminary cohort of patients with severe TBI, the CDSS was able to adequately classify the brain condition in a large proportion of time, but some errors occurred during brief transitional periods. Further training of the CDSS with a larger dataset may improve the system accuracy, which should be tested in a larger patient population. 
Poster 40

\title{
Neuro-Critical Care Services Can Manage Mild Traumatic Brain Injury Effectively Without Neurosurgical Consultation
}

\author{
Andrew Montoure, Ha H. Nguyen, Ashish A. Sharma, Vijay V. Johnson.
}

Medical College of Wisconsin, Milwaukee, WI, USA.

\section{Introduction:}

Mild traumatic brain injury (TBI) is a commonly seen pathology at trauma centers. Neurosurgical consultation is a routine practice; however, the vast majority do not require surgical intervention or invasive monitoring during the entirety of their hospital stay. In certain trauma centers, neurologycentered neurocritical care solely evaluate and manage mild TBI. We provide a retrospective analysis of this practice at our level 1 trauma center.

\section{Methods:}

A retrospective chart review was completed on TBI patients evaluated at a level 1 trauma center between September 2009 and December 2015. Inclusion criteria included; initial GCS 13-15, blunt head injury, available repeat imaging, and management by neurology-centered neuro-intensivists. Exclusion criteria included GCS $<13$, penetrating trauma, those that needed immediate surgery, those with neurosurgical consultation, and those without available imaging. A total of 87 patients were included in the final analysis.

\section{Results:}

Age was $48.8 \pm 21$ years. There were 50 males and 37 females. The most common mechanism was falls $(51.7 \%)$, followed by motor-vehicle collision $(16.1 \%)$, motor-cycle accident $(12.6 \%)$, bike-related $(8.0 \%)$, assault $(6.9 \%)$, and pedestrian-struck by automobile (4.6\%). Polytrauma occurred in $8 \%$, anticoagulation / antiplatelet (A/A) use in $23.0 \%$, and skull fractures in $41.4 \%$. On repeat CT head, $14.9 \%$ were better, $60.9 \%$ were stable, $24.1 \%$ were worse. Only 10 patients $(11.5 \%)$ exhibited neuro-exam changes, where 7 patients received repeat imaging which ultimately demonstrated stable findings. No patients required a neurosurgical procedure. Average hospital stay was $2.8 \pm 3.7$ days.

\section{Conclusions:}

Neurology-centered neuro-intensivists can manage mild TBI appropriately without official neurosurgical consultation. This practice can streamline TBI management and potentially reduce hospital costs. 


\section{Poster 41}

\section{Optic Nerve Sheath Diameter May Predict Elevations in Intracranial Pressure}

Sebina Bulic, Natasha N. Renda, May M. Kim-Tenser, Gene G. Sung, Benjamin B. Emanuel.

USC, Los Angeles, CA, USA.

\section{Introduction:}

Measurements of optic nerve sheath diameter (ONSD) using bedside ultrasound (US) have been shown to correlate with clinical and radiologic signs and symptoms of increased intracranial pressure (ICP).

\section{Methods:}

Eleven patients ( 7 males, 4 females) with traumatic brain injury (TBI) and GCS $<9$ were evaluated. All patients had extraventricular drainage (EVD) monitors, 9 right and 2 left. Three patients had right decompressive hemicraniectomy, 4 had left hemicraniectomy, 2 had suboccipital craniectomy and 2 did not have decompression. A total of 29 examinations were obtained with invasive ICP measurements, pulsatility indices (PI) using middle cerebral artery (MCA) peak systolic and end diastolic velocities and ONSD. Peak systolic and end diastolic blood pressure, temperature, serum sodium, and daily fluid balance were recorded.

\section{Results:}

Twenty-nine ocular USs were performed on 11 individual patients. In 14 ONS assessments, bilateral ONSD was $5.7 \mathrm{~mm}$, while ICP was $13 \mathrm{mmHg}$, however, later developed ICP $37 \mathrm{mmHg}$ within 24 hours. Another patient had bilateral ONS $5.8 \mathrm{~mm}$, while ICP was $16 \mathrm{mmHg}$, however, later developed ICP of $>30 \mathrm{mmHg}$ within 24 hours. Two patients had bilateral ONS measurement> $3.8 \mathrm{~mm}$ with corresponding $\mathrm{ICP}>20 \mathrm{mmHg}$

\section{Conclusions:}

All patients with ONSD20 mmHg had ONS $>5.7 \mathrm{~mm}$. Although, patients with ONS $>5.7 \mathrm{~mm}$ and ICP $20 \mathrm{mmHg}$ within the next $24 \mathrm{~h}$. There was no correlation between PI on TCD, thus TCD was not useful in this dataset. There was no correlation between increased temperature or elevated blood pressure with $I C P>20 \mathrm{mmHg}$. This small sample size suggests that ONSD may predict future ICP elevations, however, a larger sample size is needed to confirm these results. 


\title{
Poster 42
}

\section{Elevated Intracranial Pressure Causes Reversible Eye Tracking Changes Detected While Viewing Film Clip}

Molly E. Hubbard', Radek R. Kolecki ${ }^{1}$, Vikalpa V. Dammavalam ${ }^{3}$, Abdullah A. Bin Zahid ${ }^{3}$, Osama O. Chaudry ${ }^{4}$, Marlene M. Reyes ${ }^{4}$, ByoungJun B. Han ${ }^{4}$, Tom T. Wang, Vivian V. Papas ${ }^{4}$, Aylin A. Adem ${ }^{4}$, Emily E. North ${ }^{4}$, Douglas D. Kondziolka ${ }^{4}$, David D. Gilbertson ${ }^{5}$, Jason J. Huang ${ }^{4}$, Paul P. Huang ${ }^{4}$, Uzma $^{2}$ U. Samadani .

\author{
${ }^{1}$ University of Minnesota Department of Neurosurgery, Minneapolis, MN, USA, ${ }^{2}$ Department of \\ Neurosurgery, New York University School of Medicine, New York, NY, USA, ${ }^{3}$ Hennepin County Medical \\ Center Traumatic Brain Injury Research Lab, Minneapolis, MN, USA, ${ }^{4}$ Department of Neurosurgery, New \\ York University School of Medicine, New York City, NY, USA, ${ }^{5}$ Hennepin County Medical Center and \\ University of Minnesota Department of Neurosurgery, Minneapolis, MN, USA, ${ }^{6}$ Hennepin County Medical \\ Center and University of Minnesota Department of Neurosurgery, Minneapolis , MN, USA.
}

\section{Introduction:}

The precise threshold differentiating normal and elevated intracranial pressure (ICP) is variable among individuals. In the context of several pathophysiologic conditions, elevated ICP leads to abnormalities in global cerebral functioning and impacts the function of cranial nerves (CNs), either or both which may contribute to ocular dysmotility. The purpose of this study is to assess the impact of elevated ICP on eye tracking performed while watching a short film clip.

\section{Methods:}

Awake patients requiring placement of an ICP monitor for clinical purposes were eye tracked while watching a 220-second continuously playing video moving around the perimeter of a viewing monitor. Pupil position was recorded at $500 \mathrm{~Hz}$ and metrics associated with each eye individually and both eyes together were calculated. Linear regression with generalized estimating equations was performed to test the association of eye tracking metrics with changes in intracranial pressure.

\section{Results:}

Eye tracking was performed at ICP levels ranging from -3 to $30 \mathrm{~mm} \mathrm{Hg}$ in twenty-three patients (twelve female, eleven male, mean age 46.8 years) on fifty-five separate occasions. Eye tracking measures correlating with cranial nerve function linearly decreased with increasing ICP (p-value $12 \mathrm{~mm} \mathrm{Hg}$ was 0.798 .

\section{Conclusions:}

Eye tracking abnormalities detected while watching a short film clip correlate with changes in monitored intracranial pressure. Increasingly elevated ICP was associated with increasingly abnormal eye tracking. Our results suggest that eye tracking while watching a short film clip may be used to quantitate the physiologic impact of elevated intracranial pressure. This represents a new non-invasive automatable means for assessing the physiologic impact of elevated ICP. 


\title{
Poster 43
}

\section{Variable knowledge, use and perceptions of the IMPACT model among physicians during prognostication meetings for critically-ill TBI patients - results from a qualitative study}

\author{
Jesse E. Moskowitz', Thomas T. Quinn ${ }^{1}$, Lori L. Shutter ${ }^{2}$, Robert R. Goldberg ${ }^{3}$, Nananda N. Col', \\ Kathleen K. Mazor ${ }^{5}$, Susanne S. Muehlschlegel ${ }^{6}$.
}

\begin{abstract}
${ }^{1}$ University of Massachusetts Medical School, Worcester, MA, USA, ${ }^{2}$ Dept. Neurology and Critical Care, University of Pittsburgh Medical Center, Pittsburgh, PA, USA, ${ }^{3}$ Dept. Quantitative Health Sciences, University of Massachusetts Medical School, Worcester, MA, USA, ${ }^{4}$ Dept. Internal Medicine, University of New England, Biddeford, ME, USA, ${ }^{5}$ Dept. Primary Care, University of Massachusetts Medical School, Worcester, MA, USA, ${ }^{6}$ Dept. Neurology, University of Massachusetts Medical School, Worcester, MA,
\end{abstract} USA.

\section{Introduction:}

Use of shared decision-making (SDM) for goals-of-care decisions in critically-ill traumatic brain injury (ciTBI) offers the hope to decrease variation and bias in goals-of-care discussions. SDM guidelines demand the inclusion of an evidence-based outcome model, but little is known about the awareness, use, and acceptance of the "International-Mission-for-Prognosis-and-Analysis-of-Clinical-Trials-inTBI"(IMPACT)-model by physicians holding such discussions with ciTBI families.

\section{Methods:}

We conducted a mixed-methods study with semi-structured interviews in 20 attending physicians representing geographic (Northeast,Mid-Atlantic,South,West,Midwest) and subspecialty diversity (neurocritical care, neurosurgery, trauma, palliative care). We explored methods of prognosis derivation and communication, ciTBI outcome model use, and, specifically, awareness and perceived utility of the IMPACT-model. We analyzed transcripts in NVIVO-software with the investigator-triangulated-inductiveframework-approach. Theme saturation determined the final sample size.

\section{Results:}

Overall, $42 \%$ of physicians use the IMPACT-model, $25 \%$ were not aware of it, and $50 \%$ don't rely on any TBI outcome models. Positive IMPACT-model views included: "helpful in getting an idea where your confidence should be"; "to ground physicians a little bit"; "reduces the variability of prognosis that a large group of physicians may give to this very heterogeneous group of patients." Skeptical views included: "don't think the [IMPACT] data is really good," in part because TBI is a "very heterogeneous disease" that some participants "do not think has been studied very well"; "IMPACT is meant for use in research studies only"; "those calculators are about populations; they're not about individuals, use of those calculators for this purpose is a perversion of the original construct".

\section{Conclusions:}

We identified substantial physician variability in the awareness of, use, and attitude toward the IMPACTmodel, which crucially informs the development and successful implementation of future goals-of-care SDM tools in ciTBI. 


\title{
Poster 44
}

\section{The Analyses of Acute Subdural Hematoma Using Logistic Regression: The SIMC Experience}

\author{
Hiroyuki Nakajima', Hiroki H. Kurita².
}

${ }^{1}$ International Medical Center, Saitama Medical University / Cerebrovascular Surgery, Hidaka, Japan, ${ }^{2}$ International Medical Center, Saitama Medical University / Cerebrovascular Surgery, Hidaka, Japan.

\section{Introduction:}

Acute subdural hematoma (ASDH) is associated with cerebral contusion and laceration of bridging veins following a head injury, however a few cases of ASDH without head injury had been reported. The purpose of this study was to detect the difference of traumatic ASDH and non-traumatic one.

\section{Methods:}

204 cases of ASDH hospitalized at our institute from March 2014 to March 2016 were retrospectively reviewed. Traumatic groups were 189 patients (92.6\%), and non-traumatic groups were 15 patients (7.4\%). The results were statistically analyzed by logistic regression to use the various factors: age, gender, Glasgow Coma Scale (GCS) Score, the presence of light reflection, D-dimer, history of taking anti-platelet and/or anti-coagulation agents, neurological outcome and so on. Neurological outcome was evaluated using Glasgow Outcome Scale, and it was classified into two groups: the good prognosis group (GR, MD) and the poor prognosis group (SD, VS, D).

\section{Results:}

Traumatic groups were mean age $67.8 \pm 19.7$ years, and were comprised of 121 males and 68 females. Non-traumatic groups were mean age $74.5 \pm 19.5$ years, and were comprised of 6 male and 9 females. There were significant difference in patients ' characteristics, the presence of light reflection, $D$-dimer and neurological outcome $(p<0.05)$.

\section{Conclusions:}

Non-traumatic ASDH was reported to be rare and poor prognosis, but it was not known to clinical feature. We will be described the detail of them and collect further cases in the future. 


\title{
Poster 45
}

\section{Clinical Profile And Predictors Of In-Hospital Mortality In Traumatic Brain Injuries: Urban Indian Experience}

\author{
Deepak R. Jeswani ${ }^{1}$, Monika M. Mandal' ${ }^{2}$ Dilip D. Kshirsagar ${ }^{2}$, Shirish S. Mahure ${ }^{2}$, Rupali R. Bhanare ${ }^{2}$, \\ Chitra C. Pardhi', Vijay V. Katkhaye ${ }^{3}$. \\ ${ }^{1}$ Criticare Hospital \& Reaserch Institute, Nagpur, India, ${ }^{2}$ Criticare hospital and Research Institute, Nagpur, \\ India, ${ }^{3}$ Dev Clinic, Nagpur, India.
}

\section{Introduction:}

Traumatic brain injuries (TBI) are of significant importance due to increased morbidity and mortality. We retrospectively analysed TBIs to assess clinical profile and factors predicting in-hospital mortality.

\section{Methods:}

Electronic database at a private, urban tertiary care centre was screened (2011 to 2016) to include all TBI patients $\geq 12$ years. Total 120 patients were analysed and factors predicting in-hospital mortality outcome were assessed.

\section{Results:}

Mean age of population was $39.2 \pm 17.2$ years with $81.8 \%$ being males. Most injuries $(90 \%)$ resulted from road traffic accidents (RTAs). Bleeding from either ear, nose or throat (ENT) was most common presentation $(29.2 \%)$ followed by vomiting $(25.8 \%)$ and convulsions $(7.5 \%)$. On presentation, severe brain damage was evident in $21.7 \%$ (Glasgow coma scale (GCS) score $\leq 8$ ) and tachycardia in 30\% patients. Subdural $(36.7 \%)$ haematoma was most common followed by sub-arachnoid $(25.8 \%)$, extradural $(16.7 \%)$ and intracerebral (2.5\%). Threatened airway was observed only in $7.5 \%$ cases. Bony trauma $(25 \%)$ followed by face $(14.2 \%)$, chest $(3.3 \%)$, and spine injuries $(0.8 \%)$ were associated injuries. In-hospital mortality was $13.3 \%$ during a mean hospital stay of $8.05 \pm 7.5$ days (range: 1 to 47 days). Increased risk of in-hospital mortality was associated with GCS $\leq 8$ (odd ratio (OR) 15.67, 95\% confidence interval $(\mathrm{Cl})$ 4.82, 50.88; $p<0.0001$ ), tachycardia (OR 3.0, 95\% Cl 1.21, 7.43; $p=0.014$ ) and with development of hyponatremia (OR 5.0, 95\% Cl 2.17, 11.53; $p<0.0001$ ) or fever (OR 3.18, 95\% Cl 1.29, 7.61; $\mathrm{p}=0.010$ ) during hospitalization. Ventilator support was necessary in 29 (23\%) cases out of which 16 (55\%) died. Hospital stay (days) did not vary significantly in survivors and non-survivors (5.5 \pm 4.0 Vs $8.5 \pm 7.8$, $\mathrm{p}=0.156)$.

\section{Conclusions:}

TBls were result of RTAs which ranged from mild to severe. GCS $\leq 8$, tachycardia on presentation, development of hyponatremia or fever and requirement of assisted ventilation were associated with increased risk of in-hospital mortality. 


\title{
Poster 46
}

\section{Implementation of a Daily Goals Tool Improves Team Communication Surrounding Quality and Safety Practices in a Neurosciences Intensive Care Unit}

\author{
Casey Olm-Shipman ${ }^{1}$, Megan M. Brissie ${ }^{2}$, Marin M. Darsie ${ }^{3}$, Rhonda R. Cadena ${ }^{3}$, Sharmila S. Soares- \\ Sardinha ${ }^{4}$, Christa C. Williams ${ }^{4}$, Emily E. Durr ${ }^{5}$, Kelly K. Sullivan ${ }^{5}$, Kathryn K. Morbitzer ${ }^{5}$, Denise D. \\ Rhoney ${ }^{5}$, Dedrick D. Jordan 6 .
}

\begin{abstract}
${ }^{1}$ University of North Carolina at Chapel Hill School of Medicine, Department of Neurology, Chapel Hill, USA, ${ }^{2}$ University of North Carolina at Chapel Hill, Department of Neurology, Chapel Hill, USA, ${ }^{3}$ University of North Carolina at Chapel Hill, Chapel Hill, USA, ${ }^{4}$ University of North Carolina, Department of Neurology, Chapel Hill, USA, ${ }^{5}$ University of North Carolina, School of Pharmacy, Chapel Hill, USA, ${ }^{6}$ University of North Carolina, Chapel Hill, USA.
\end{abstract}

\section{Introduction:}

Communication errors remain leading causes of medical errors. Daily Goals Tools (DGT) are team communication aids associated with reductions in hospital-associated morbidities. We implemented a DGT to prompt standardized team communication of quality and safety indicators (QSI) during rounds in the Neuroscience Intensive Care Unit (NSICU).

\section{Methods:}

We established a team that included attending physicians, fellows, advanced practice providers (APP), nurses, respiratory therapists and pharmacists. We defined key QSIs from national, institutional, and unit specific guidelines. We surveyed staff regarding team communication and discussion of QSIs during rounds. We designed a DGT that defined team member roles, structured communication patterns, and prompted standard discussion of QSIs. Following implementation, we evaluated team compliance with the DGT, as well as rates of catheter-associated urinary tract infections (CAUTI) and ventilatorassociated pneumonias (VAP).

\section{Results:}

61 NSICU staff participated (66\% nurses, $20 \%$ physicians/advanced practice providers, $3 \%$ pharmacists, $11 \%$ respiratory therapists). For most QSIs, a minority of staff reported that these were always addressed during morning rounds (\% staff indicating QSI "always" addressed: $14 \%$ pressure ulcers, $19 \%$ code status, 33\% CAM ICU, 36\% mobility goal, $46 \%$ central line catheter removal, $50 \%$ urinary catheter removal, $66 \%$ DVT prophylaxis plan, $69 \%$ ventilator weaning). Shared understanding of daily goals between nurses and physicians was reported by $58 \%$ of staff, with a significant difference between nurse and physician/APP respondents ( $66 \%$ vs $33 \%$ ), $p=0.047$. DGT audits spanning 212 patient days demonstrated median compliance greater than $80 \%$ for discussion of all QSIs. There was an $11 \%$ reduction in CAUTIs $(95 \% \mathrm{Cl} 3 \%, 19 \%), p=0.011$, and a trend towards reduction in VAP that was not significant.

\section{Conclusions:}

Team discussion of QSIs can be enhanced by DGTs. By promoting adherence to evidence-based best practice, DGTs may reduce hospital-associated infections. Follow-up is ongoing to determine the impact of DGTs on clinical outcomes and team communication. 


\title{
Poster 47
}

\section{Mission Impossible: Achieving Zero CAUTIs in the Neurolntensive Care Unit Using a Patient- Centered, Interdisciplinary Team Approach}

\author{
Jeannette M. Hester ${ }^{1}$, Karen K. Perrin ${ }^{2}$, Richard R. Reed ${ }^{2}$, Angela A. Larson ${ }^{3}$, Brenda B. Fahy ${ }^{4}$, Kim K. \\ Browning ${ }^{5}$, Jacqueline J. Baron-Lee 6 .
}

${ }^{1}$ UF Health Shands Hospital- Neurolntensive Care Unit, Gainesville, FL, USA, ${ }^{2}$ UF Health Shands Hospital- NeurolCU, Gainesville, FL, USA, ${ }^{3}$ UF Health Shands Hospital-Department of Nursing and Patient Services, Gainesville, FL, USA, ${ }^{4}$ University of Florida College of Medicine, Department of Anesthesiology, Gainesville, FL, USA, ${ }^{5}$ UF Health Shands- Department of Infection Control, Gainesville, FL, USA, ${ }^{6}$ University of Florida, Department of Neuromedicine, Gainesville, FL, USA.

\section{Introduction:}

Catheter-associated urinary tract infection (CAUTI) is the most common health-care associated infection accounting for $>560,000$ nosocomial infections annually (Gould,2016). According to the Center for Disease Control, CAUTIs are also a leading cause of secondary blood stream infection resulting in approximately 13,000 deaths annually. The neurocritical care population is especially at risk for CAUTI development related to cognitive, motor, and sensory deficits. NeurolCU's goal was to eliminate CAUTIs, defined as a rate of zero. With Initial efforts (2008-2012), CAUTI rate was reduced from 13.1 to 4.0/1000 catheter days (Titsworth et al, 2012). In February, 2016 NeurolCU launched a patient-centered quality improvement effort to further reduce CAUTIs.

\section{Methods:}

The NeuroICU Interdisciplinary Comprehensive Unit Safety Program (CUSP) performed an extensive literature review of evidence based best practices specific to urinary catheter management. A preexisting, staff nurse driven urinary catheter management protocol was revised to better fit the needs of the neurocritical care population, including assessment of and interventions for acute and chronic retention, specified frequency for voiding/bladder scan assessments to every 4 hrs, revision of urinary catheter criteria (e.g. postop monitoring and hemodynamic instability $>24 \mathrm{hrs}$, where intake/output is actively used to drive interventions), unstable spinal fracture, and patient/family request during end-oflife/palliative care). NeurolCU's nursing leadership and the neurocritical care interdisciplinary team conducted daily rounds to evaluate the necessity and management of indwelling urinary catheters.

\section{Results:}

After three months of implementation, compliance with use of the NeurolCU's Urinary Catheter Management Algorithm (UCMA) was greater than 95\%, urinary catheter utilization was reduced from $65 \%$ to $38 \%$, and CAUTI rates were reduced to zero.

\section{Conclusions:}

Implementing a neurocritical care patient-centered, interdisciplinary approach to urinary catheter management significantly impacted urinary catheter utilization, CAUTI rates, and unit culture. 


\title{
Poster 48
}

\section{Improvement In Quality Outcome Measurements in Neurosciences Intensive Care Unit After Appointment of Neurointensivists}

\author{
Yaw Sarpong ${ }^{1}$, Ginger G. Schelp ${ }^{2}$, Robert R. Bell ${ }^{1}$, Erin E. Stapleton ${ }^{2}$, Karen K. Cox ${ }^{2}$, Ashley A. Ausmus ${ }^{3}$, \\ Pradeep P. Sahota ${ }^{4}$, N. Scott N. Litofsky ${ }^{1}$, Premkumar P. Nattanmai ${ }^{4}$, Christopher C. Newey ${ }^{4}$. \\ ${ }^{1}$ University of Missouri, Division of Neurosurgery, Columbia, MO, USA, ${ }^{2}$ University of Missouri, Columbia, \\ MO, USA, ${ }^{3}$ University of Missouri, Department of Pharmacy, Columbia, MO, USA, ${ }^{4}$ University of \\ Missouri, Department of Neurology, Columbia, MO, USA.
}

\section{Introduction:}

Dedicated neurointensivists have previously been shown to improve various outcome measurements in the neurosciences intensive care unit (NSICU). These studies lack quality outcome data assessing patient and family satisfaction. The purpose of this study is to evaluate the impact of newly appointed neurointensivists on quality outcome measures in a NSICU.

\section{Methods:}

This is an observational cohort study of adult patients (>18 years) in a 10-bed NSICU at an academic, tertiary care center evaluating quality outcome measures pre- and post-neurointensivists. Outcome measurements include catheter associated urinary tract infection (CAUTI), central line blood stream infection (CLABSI), ventilator associated pneumonia (VAP), patient acuity, mortality, and length of stay (LOS). Patient satisfaction questionnaires from discharged patients were compared to historical controls. The questionnaire with benchmarks include: 1) Doctors/Nurses consistency; 2) Confidence/trust in doctors; 3) Courtesy/respect by doctors; 4) Attentiveness of doctors; 5) Recommend the hospital to others. Statistics include 1-sample binomial and N-1 Chi-squared (categorical) and t-test (continuous). For questionnaire data, 1-sample binomial test was performed using benchmark of $70 \%$. $p<0.05$ was considered significant.

\section{Results:}

2547 total patient days occurred pre- and 2560 days post-neurointensivist coverage. Patient acuity increased $3.5 \%(p=0.041)$ while LOS decreased $2.7 \%(p=0.612)$. Neurology/neurosurgery mortality decreased $24.2 \%(p=0.043)$. CAUTI $(66 \%, p=0.084)$, CLABSI $(100 \%, p=0.32)$, central line days $(16.5 \%$, $p<0.0001)$, ventilator days $(18.7 \%, p=0.3)$, and VAP $(200 \%, p=0.15)$ also decreased. These saved the hospital an estimated $\$ 65,000$ based on Health Services Advisory Group Data. 89 questionnaires were returned. Patient satisfaction increased $7 \%$ on doctors/nurses consistency $(p=0.03), 86 \%$ in confidence/trust in doctors $(p=0.03), 86 \%$ on doctors treated me with courtesy/respect $(p=0.03)$, and $64 \%$ on physicians' attentiveness $(p=0.03)$. Patients recommending the hospital to others increased $67 \%$ $(p=0.03)$.

\section{Conclusions:}

Dedicated neurointensivists positively impact quality outcome metrics, particularly significantly improving patient satisfaction. Future studies should evaluate the direct impact of neurointensivists on Medicare reimbursement from improved patient satisfaction. 


\title{
Poster 49
}

\section{Noninvasive Continuous Imaging of Reduced Cerebral Perfusion with a Novel Diffuse Optical Tomography System: A Preliminary Hyperventilation Study in Pig and Human}

\author{
Bertan Hallacoglu, Tanmayi T. Oruganti, Chandran C. Seshagiri.
}

Research \& Development, Boston, MA, USA.

\section{Introduction:}

Cephalogics has developed a wearable Diffuse Optical Tomography (DOT) imaging device to help clinicians monitor perfusion and oxygenation from multiple brain regions on the bedside in disease states such as ischemic stroke, vasospasm, and traumatic brain injury. This study investigated the sensitivity of the system to changes in cerebral tissue oxygenation (SctO2) induced by hyperventilation in a pig and human subjects.

\section{Methods:}

A 45kg Yorkshire pig was placed in prone position, anesthetized, and mechanically ventilated. The first DOT sensor was positioned on the pig's head along the sagittal line, and the second sensor was positioned on the hind leg muscle for monitoring systemic tissue oxygenation (SstO2). DOT measurements were performed continuously during baseline ( $8 \mathrm{mins}, \mathrm{PaCO} 2=40 \mathrm{mmHg}$ ), transient hypercapnia ( 4 mins, $\mathrm{PaCO} 2=15 \mathrm{mmHg}$ ), and recovery $(25 \mathrm{~min}, \mathrm{PaCO} 2=40 \mathrm{mmHg})$ periods controlled by the respiratory rate of the ventilator. DOT data were recorded to a laptop for off-line analysis. StO2 images were reconstructed every 1 minute, and image means (+/-SD) were computed for comparison of results across measurements. The approach was also investigated in three human volunteers, who were instructed to hyperventilate during DOT recordings.

\section{Results:}

SctO2 estimates in pig markedly decreased during hyperventilation from baseline $(-15 \%+/-6 \%)$, consistent with reports of reduced cerebral blood flow during hypocapnia. In contrast, SstO2 estimates modestly increased during hyperventilation $(+3+/-1 \%)$, indicating elevated systemic perfusion. Both parameters fully recovered to baseline values during the recovery period. SctO2 response to hyperventilation in human volunteers were consistent with the results in pig.

\section{Conclusions:}

Hypercapnia induced reduction in SctO2 was noninvasively imaged in human subjects and a pig despite the large scalp-cortex distance in pig. The results of this study demonstrate the sensitivity of the Cephalogics' DOT system to SctO2 values and its ability to separate SctO2 from systemic perfusion. 


\title{
Poster 50
}

\section{Electronic (EPIC) Tool Facilitates Nurse Handover and Nurse-Led Neurocritical Care Rounds}

\author{
Brittany Doyle, Michael M. Rogers, DaiWai D. Olson, Venkatesh V. Aiyagari.
}

UT Southwestern, Depts. of Neurological Surgery, Neurology and Neurotherapeutic, Dallas, USA.

\section{Introduction:}

Multidisciplinary rounds play a pivotal role in optimizing care in the Neurosciences Critical Care Unit (NCCU). Care providers were frustrated with inconsistency and the need for manual data entry to conduct multidisciplinary rounds. The purpose of this project was to develop an integrated computerized form that incorporated "smart" features within EPICTM.

\section{Methods:}

The NCCU Nursing Council collaborated with the physicians to design a standardized system-based approach to multidisciplinary rounds, and the elements that would be addressed within each system. Input was collated from nursing, neurology, neurosurgery, pharmacy, and critical care into a paper version which was beta tested before the EPIC tool was officially rolled out.

\section{Results:}

The rounds template is now a perpetually editable note with fields that include drop-down menus, copyforward fields, and key fields that auto-populate with up-to-date data (e.g., ICP values, lab values). While there was a learning curve to the use and unanimous adoption of the tool, within a few weeks, nurse-led rounds became much more efficient, comprehensive, and less frustrating for the entire healthcare team. Within four months, nursing management saw the benefit of such a standardized tool for clear communication and the tool also became the standard for nurse-to-nurse handover at change of shift.

\section{Conclusions:}

Efficient and orderly presentation of information during multidisciplinary rounds is greatly assisted by the use of a standardized electronic tool. Having all of the current, relevant data available in a single location has greatly improved the quality of nurse-led Neurocritical Care rounds. This tool can be replicated and customized to the needs and patient populations of other units and hospitals. 


\title{
Poster 51
}

\section{The Use of Quantitative EEG Burst Suppression Ratio For Evaluating Depth of Pharmacologic Coma}

\author{
Christa B. Swisher ${ }^{1}$, Kingshuk K. Roy Choudhury², Suarabh S. Sinha'. \\ ${ }^{1}$ Duke University Medical Center / Department of Neurology, Durham, NC, USA, ${ }^{2}$ Duke University Medical \\ Center / Department of Biostatistics and Bioinformatics, Durham, NC, USA.
}

\section{Introduction:}

The degree of burst suppression on continuous critical care EEG (CCEEG) monitoring is used to guide dosing of intravenous anesthetic drugs (IVADs) in the treatment of refractory elevated intracranial pressure (ICP) and refractory status epilepticus (SE). However, medication titration is performed only as frequently as CCEEG review (potentially as little as 2-3 times a day). Quantitative EEG (QEEG) may provide a continuous, objective assessment of the level of burst suppression that would allow for more precise and rapid titration of IVADs.

\section{Methods:}

Nine patients with refractory ICP and/or SE treated with IVADs were studied retrospectively. We compared software-generated QEEG burst suppression ratio (QEEG BSR) with three raw EEG variables as determined by two board-certified neurophysiologists (R1 and R2): bursts per minute (BPM), total burst duration per minute (BD) and average inter-burst interval (IBI) for 10 one minute segments per patient. $A$ total of 90 EEG segments were analyzed.

\section{Results:}

Agreement between readers was very high for the three raw EEG variables: BPM, BD and IBI (correlation coefficient 0.92, 0.96, and 0.95, respectively). The best correlation was observed between BD and QEEG BSR (-0.93 for both R1 and R2). The correlation between BPM and QEEG BSR (-0.80 R1 and -0.75 R2) and IBI and QEEG BSR (0.70 R1 and 0.65 R2) was not as strong. Left and right hemispheric QEEG BSR did not differ statistically from the generalized QEEG BSR ( $p=0.11$ and 0.08 , respectively) despite the presence of focal intracranial pathology.

\section{Conclusions:}

The depth of therapeutic burst suppression can be accurately assessed by generalized QEEG BSR. Although CCEEG bursts per minute is the most commonly used CCEEG metric of burst suppression, it shows a lesser correlation with QEEG BSR than total burst duration per minute. 


\title{
Poster 52
}

\section{Optimal Neurosciences Intensive Care Unit (Neuro - ICU) Staffing Models: Addition of Night-time In-hospital Neurocritical Care Fellows Correlates with Nursing, Physician, Non-physician Provider, and Patient/Surrogate Satisfaction}

\author{
Agnieszka Ardelt ${ }^{1}$, An A. Zhang ${ }^{2}$, Anantha A. Vellipuram ${ }^{2}$, Raisa R. Martinez ${ }^{2}$, Tania T. Rebeiz ${ }^{2}$, Deb D. \\ Mojumder ${ }^{2}$, Christopher C. Kramer ${ }^{2}$, Fernando F. Goldenberg ${ }^{2}$. \\ ${ }^{1}$ University of Chicago/Neurology; Surgery, Chicago, IL, USA, ${ }^{2}$ University of Chicago/Neurology, Chicago, \\ IL, USA.
}

\section{Introduction:}

There is little operational data on optimal Neuro-ICU physician staffing. This prospective study evaluated the introduction of night-time in-hospital neurocritical care fellows (NCFs) in an urban academic hospital. The goal was to determine if the new staffing model enhances patient care and provider and patient satisfaction.

\section{Methods:}

IRB approval was obtained. The new staffing model was rolled out on 9-1-2015. 121 providers (NCFs; neurology residents and attendings; neurosurgery residents, physician assistants, advanced practice nurses and attendings; Neuro-ICU nurses) were surveyed. A pre-roll out survey of providers' perceptions of the existing model (night-time NCF coverage from home with on-call in-hospital neurology residents and neurosurgery physician assistants or residents, not dedicated to the Neuro-ICU) was administered prior to new model roll-out. Two follow-up surveys, a separate night-shift nurses survey, and patient (or surrogate) surveys were administered between 9-1-15 and 5-10-16. Surveys were electronic and responses were voluntary and anonymous except for the night-shift nurses survey which was paper, required, and anonymous.

\section{Results:}

Response rates were $52 \%$ (pre-roll out), $28 \%$ (first follow-up), and $25 \%$ (second follow-up). $93 \%$ of providers indicated pre-roll out that night-time in-hospital NCFs would be beneficial; $94 \%$ (first follow-up) and $92 \%$ (second follow-up) indicated satisfaction with the new staffing model. Major reasons for satisfaction included: physician response to emergencies, physician - nurse communication, and patient outcome. The reason for dissatisfaction was decreased educational opportunity for residents. $94 \%$ of night-shift nurses reported preference for the new model. $62 \%$ of 72 patients (or surrogates) agreed to participate: $87 \%$ reported satisfaction with the care they received in the ICU; dissatisfaction was not linked to the staffing model.

\section{Conclusions:}

More than $92 \%$ of providers were satisfied with a new staffing model featuring night-time in-hospital NCFs. Additional studies are necessary to determine optimal Neuro-ICU physician staffing with increasing patient and treatment complexity and decreasing resources. 


\title{
Poster 53
}

\section{A Neurointensivist Managed Plasma Exchange Program}

\author{
Paul J. McCarthy ${ }^{1}$, Micheal M. Armahizer ${ }^{2}$, Melissa M. Motta ${ }^{3}$,Wendy W. Chang ${ }^{3}$, Gunjan G. Parish ${ }^{3}$. \\ ${ }^{1}$ University of Maryland Medical Center, Program in Trauma, Baltimore, MD, USA, ${ }^{2}$ University of Maryland \\ Department of Pharmacy, Baltimore, MD, USA, ${ }^{3}$ University of Maryland, Program in Trauma, Baltimore, \\ $M D, U S A$.
}

\section{Introduction:}

Therapeutic plasma exchange (TPE) is a first-line therapy for Guillain-Barre syndrome, myasthenia gravis, chronic inflammatory demyelinating polyneuropathy and polyneuropathy associated with paraproteinemias. TPE is also a second-line therapy for neuromyelitis optica, chronic focal encephalitis, and acute multiple sclerosis. The therapy is also used in autoimmune encephalopathies, refractory status epilepticus, heparin induced thrombocytopenia, and in septic shock. Since April 2015 our neurocritical care service has provided a neurointensivist run TPE program using membrane-based technology with the Gambro® Prismaflex System. A benefit of a neurointensivist managed membrane - based service is lower direct costs compared to centrifugal- based therapies. Since a consultation to another service (often available during business hours) is avoided, delays in therapy can be reduced (potentially further decreasing costs) and the therapy is also available for emerging indications such as refractory status epilepticus.

\section{Methods:}

This review is of the initial 15 patients treated with TPE in our Neurocritical Care Unit between April 2015 and May 2016. All patients were treated with $5 \%$ albumin with a targeted exchange of 1.2 to 1.5 plasma volumes. Overall dosing, timing, indications as well complication were reviewed.

\section{Results:}

Indications for therapy included Guillain-Barre syndrome, Myasthenia Gravis, autoimmune encephalitis, refractory status epilepticus, autoimmune vasculitis, and parainfectious transverse myelitis. All patients received a minimum prescription of 1.2 plasma volumes and had the therapy started sooner than historical controls. No changes in nurse staffing were required for the therapies and we report no bleeding, infectious or access related complications. Mild coagulopathy was seen in most patients after therapy. Many patients' therapies were completed at night and/or weekends when the therapy was previously not available.

\section{Conclusions:}

A neuro-intensivist lead TPE program is feasible and safe. The therapy can be delivered more timely and can be offered for a wider variety of indications. A cost analysis of the program is now underway. 


\title{
Poster 54
}

\section{Outcome Following Acute Neurological Injury: A Pilot Feasibility Study}

\author{
Erika Schlichter, BSN RN ${ }^{1}$, Heather H. Oehler, BSN RN ${ }^{1}$, Alisha A. Hodge, BSN RN ${ }^{2}$, Kelly K. Rath, MS \\ $A C N P^{3}$, Daniel D. Kanter, $M D^{3}$, Christopher C. Zammit, $M D^{4}$, Natalie N. Kreitzer, MD ${ }^{4}$, Simona S. Ferioli, \\ $M D^{3}$, Brandon B. Foreman, $M D^{3}$.
}

\begin{abstract}
${ }^{1}$ University of Cincinnati, Neuroscience Intensive Care Unit, Cincinnati, OH, USA, ${ }^{2}$ University of Cincinnati, Department of Neurology \& Rehabilitation Medicine, Cincinnnati, OH, USA, ${ }^{3}$ University of Cincinnati, Department of Neurology \& Rehabilitation Medicine, Cincinnati, $\mathrm{OH}, \mathrm{USA},{ }^{4}$ University of Cincinnati, Department of Emergency Medicine, Cincinnati, OH, USA.
\end{abstract}

\section{Introduction:}

Specialty neurocritical care improves hospital length of stay and mortality in patients with critical neurologic illness. However, clinical practices are often informed by studies focused on mortality or gross functional outcome. Both staff and family members face uncertainty about neurological outcome, which affects medical decision making. We sought to demonstrate the feasibility of performing detailed followup for all patients admitted to the University of Cincinnati Neurosciences Intensive Care Unit (NSICU).

\section{Methods:}

All patients admitted during a two-week period to the NSICU for $>24$ hours were identified. Medically trained staff (MD/RN) called patients using a scripted case report form at 3 months following admission. We assessed functional outcome using the modified Rankin scale (mRS) and the revised Glasgow Outcome Scale-Extended (GOSE), cognitive outcome using the Telephone Interview for Cognitive Skills, and quality-of-life using the Euro-QOL. We assessed the duration of each follow-up call in order to determine feasibility.

\section{Results:}

$\mathrm{n}=50$ patients were admitted to the NSICU; mean age was $59 ; 63 \%$ were male. Overall follow-up was obtained in $80 \% ; 64 \%$ of survivors were contacted. Disposition from NSICU included: home (20\%), acute rehab $(27 \%)$, long-term care $(24 \%)$, hospice (4\%). In-hospital mortality was $16 \%$. Median GOSE (IQR) was 4 (3-7) and median mRS (IQR) was 3 (1-4). 15/27 were assessed for cognition; $40 \%$ had cognitive impairment. The EuroQOL visual analog scale was 53+/-30. An average of 13:28 (mm:ss) was required for each assessment; comprehensive assessments required 21:54. Overall, a total time of approximately 11 hours was required for 50 patients.

\section{Conclusions:}

Our pilot study demonstrated feasibility of following patients admitted to the NSICU. These findings have the potential to guide in-hospital care and out-of-hospital resources when used as a quality improvement metric, and to provide valuable information for retrospective research. 


\title{
Poster 55
}

\section{Staffing is More Than a Number: Using Workflow to Determine an Appropriate Nurse Staffing Ratio in a Tertiary Care Neurocritical Care Unit.}

\author{
Michelle Hill, Stormy S. Dulovich.
}

Riverside Methodist Hospital/Neurocritical Care, Columbus, OH, USA.

\section{Introduction:}

Our Neurocritical Care Unit restructuring provided an opportunity to revise our staffing model. The prior model ratio of $1: 2$ resulted in at least one nurse experiencing a 1:3 ratio when high acuity patients required 1:1 nursing care. Yet, we lacked adequate unit-specific data to support a higher staffing.

\section{Methods:}

Prospective observational study of nursing time using the 28-item Therapeutic Intervention Scoring System (TISS-28) and manual timing of discrete nursing interventions including off-unit transport. Baseline data was obtained to include measures of nursing experience.

\section{Results:}

Measures of central tendency and regression analysis was performed using SAS v9.4. The average time to complete a neurologic assessment was 4.17 minutes; the average time to chart a neurologic assessment was 4.05 minutes. For time spent off the unit the average time spent traveling to CT was $<20$ minutes, average time spent in MRI was 80 minutes twice a day, average time in IR was 82.5 minutes. We found that nursing experience was not associated with patient acuity, duration of assessment, nor time spent documenting. Nursing experience is not associated with how long it takes to do an assessment. Nursing experience was not associated with how long it took to chart, however, when you factor in acuity the novice nurses took longer to chart on higher acuity patients. Assessment time was associated with documentation time. Moreover, higher acuity was a predictor of both assessment time and documentation time.

\section{Conclusions:}

The data were used to support an increased acuity model with 3 nurses having planned 1:1 ratio. Neurologic critical are patients require more hands on nursing care and time spent in diagnostic testing than an average critical care patient. Neurocritical Care Units staffing ratios should be adjusted to provide time to ensure nursing care is complete. 


\title{
Poster 56
}

\section{Algorithm for Determining Venous Access Device in Neurocritical Care}

\author{
Melissa Panter, Sonja S. Stutzman, DaiWai D. Olson, Venkatesh V. Aiyagari.
}

UTSouthwestern/Neurocritical Care, Dallas, USA.

\section{Introduction:}

Venous access is a basic yet critical component of care. Determining which venous access devices (VAD) to use depends on the type, duration, and frequency of infusion. Prolonged continuous infusion of drugs or fluids, benefit from midline peripheral catheter (midline) or a peripherally inserted central catheters (PICC). Midlines are safe and effective but use is declining in favor of PICCs, which have similar insertion costs and added benefits, such as the delivery of toxic drugs harmful to peripheral veins. However, an infection associated with a PICC is classified as a central line associated blood stream infection which has important financial implications for the hospital. Therefore, it is important that the type of VAD be specifically tailored based on the indication. We embarked on a nurse-physician collaborative study to design and test a decision support tool to assist clinicians in determining the appropriateness of the PICCs vs midlines for patients.

\section{Methods:}

This study has three phases. First, a retrospective chart review of VAD decision-making practices to examine the current degree of appropriateness for each type of VAD for each specific patient scenario. Next, we will design a decision support tool to help decide which VAD should be used. The tool will be developed based on expert clinician, pharmacist review and a review of the literature. Finally, we will prospectively evaluate the efficacy of this tool to guide clinicians in making the correct choice of VAD for their patients.

\section{Results:}

The first phase of the study is ongoing and we will present the results of the retrospective part of the study and the decision support tool at the annual Neurocritical Care Society meeting.

\section{Conclusions:}

A decision support tool to guide clinicians choosing between different types of VAD will help improve current clinical practice and patient outcomes. 


\title{
Poster 57
}

\section{A Conceptual Framework for a Technical Interoperability Standard Promoting Highly Efficient Network-Based Clinical Trials and Collaborative Research}

\author{
Eric S. Rosenthal ${ }^{1}$, M. Brandon M. Westover ${ }^{1}$, Anna A. Rodriguez ${ }^{2}$, Dick D. Moberg ${ }^{2}$. \\ ${ }^{1}$ Massachusetts General Hospital, Department of Neurology, Boston, MA, USA, ${ }^{2}$ Moberg Research, Inc. , \\ Ambler, PA, USA.
}

\section{Introduction:}

Clinical trials in neurocritical care require a predictable set of baseline, monitoring, treatment, and clinical outcomes data. However, interoperability standards restrict automated real-time streaming of this data, resulting in inefficiencies performing clinical trials, preventing real-time clinical trial oversight and constraining collaborative research. We investigated available data systems and developed a conceptual framework for clinical trial oversight and collaborative research in neurocritical care.

\section{Methods:}

Examining current traumatic brain injury interventional trials, we considered technical interoperability standards and data flow elements required for a system capable of network-based clinical trial oversight and collaborative analytic research. We elaborated a vendor-neutral interoperability schema for data extraction, repositories, analysis, annotation, and visualization.

\section{Results:}

The proposed conceptual solution is described. Nodes of data acquisition include: 1) continuously streaming devices including physiologic monitors and infusion pumps; 2) discrete data from highpenetrance EHR and laboratory platforms; and 3) biospecimen, radiology, and clinical outcomes repositories. An application program interface performs function calls to utilize individual episodes of data. A data management system queries and manages multiple patient records for batch processing of ondemand or pre-specified queries. A user interface toolkit enables annotation, analysis, and visualization for real-time or post-hoc assessment of raw and derived parameters (e.g., percent time in target range or on-protocol compliance). We enumerate the variety of current nodes requiring interoperability interfaces, and propose an open standard to promote a highly efficient platform for network-based clinical research, featuring automated case report form data extraction, a programmable interface for oversight and early warning detection, and a platform for annotation and crowdsourcing of novel algorithms.

\section{Conclusions:}

This conceptual architecture for a modular, vendor-neutral, data collection and management system for the acute care of patients in neurocritical care offers scalable efficiencies that promote network-based clinical trials in neurocritical care and offer new functionality for real-time oversight and collaborative analytics. 


\section{Poster 58}

\section{Improving Patient Outcomes: The Impact of Creating a Specialized Neuro-Oncology Critical Care Unit}

Shannon M. Appelfeller, Monica M. Arce.

The James Cancer Hopsital \& Solove Research Institute - James Neurocritical Care Unit, Columbus, OH, USA.

\section{Introduction:}

Cancer is a devastating illness; with a rise in brain and spine tumors specialized care is more important that ever. With advances in technology and treatment strategy, those too ill to previously receive care now actively seek treatment. The result of increased acuity has translated to the need for higher levels of hospital care. Recognizing the unique expertise required to care for this emerging population, the decision was made to merge neurology and oncology specialties within critical care. The purpose of this poster is to describe the admission criteria, patient population, utilized technology, staffing model, and patient outcomes of a newly created neuro-oncology critical care unit (NCCU).

\section{Methods:}

In January 2014, a 24 bed neurocritical care unit was opened in a midwestern academic medical center. Six of the beds housed within this 24 bed unit were designated and budgeted to the oncology medical center. Separate nursing staff and management teams were created to support the care within the unit while maintaining close collaboration with university hospital NCCU nursing teams. While nursing teams are distinct, nurse practitioners, neurointensivist, and other multidisciplinary team members are shared between both services. To evaluate the benefit of establishing this type of unit the hospital tracked, patient diagnosis, care needs, NCCU length of stay (LOS), number of ventilator acquired pneumonias (VAPs), urinary tract infections (UTIs), and central line acquired bloodstream infections (CLABSIs).

\section{Results:}

At the time of this submission, specific results are still being tabulated.

\section{Conclusions:}

Merging access to academic research trials and oncologic and neurointensive specialists, this has created an environment that promotes care reflective of the most up to date evidence based practice. Through the utilization of creative staffing and focused onboarding, this unit has been able to treat this subspecialized population holistically utilizing a multidisciplinary approach to minimize hospital acquired complications and LOS. 


\title{
Poster 59
}

\section{Recommendations for Medical Device Connectivity: Turning the NCS Multimodal Monitoring Consensus Statement into Action}

\author{
Anna Rodriguez ${ }^{1}$, Dick D. Moberg ${ }^{2}$, Eric E. Rosenthal ${ }^{3}$. \\ ${ }^{1}$ Director of Research, Moberg Research, Inc., Ambler, PA, USA, ${ }^{2}$ Moberg Research, Inc., Ambler, PA, \\ USA, ${ }^{3}$ Massachusetts General Hospital, Assoc. Dir. MGH Neurosciences ICU, Boston, MA, USA.
}

\section{Introduction:}

In 2014, the Neurocritical Care Society (NCS) published a consensus statement in support of multimodal neuromonitoring, emphasizing the essential need for systems to integrate data in meaningful ways to support decision making in patient care. A significant barrier to the integration of data from multiple sources is the lack of standards for medical device data.

\section{Methods:}

In order to accelerate the development of a "connected environment" in neurocritical care, the Working Group on Neurocritical Care Informatics was established in 2015; it includes experts from the clinical and research side of neurocritical care and representatives from medical device manufacturers. Through an open meeting and continued discussion, a recommendation document "Medical Device Connectivity" was produced. Its objective is to provide guidance to medical device manufacturers desiring to design a communication protocol that allows external systems to acquire data from their devices.

\section{Results:}

Our research on this project uncovered a high percentage of errors in medical device protocols as well as undocumented characteristics and safety issues. The group developed recommendations for providing robust communications as well as address usability and safety concerns. The document outlines the content that should be transmitted which includes device identification, protocol version identification, patient identifier, events, alarm conditions, system status, data labels, and units. Finally, it must be thoroughly documented and validated by the manufacturer.

\section{Conclusions:}

Multimodal monitoring (which requires medical device connectivity) reportedly improves quality of care through reduction of errors and increased detection of adverse events. However, widely adopted communications standards that facilitate these benefits are lacking. Updated standards for device connectivity are on the horizon. In the meantime, the document created in this project will provide guidance for manufacturers in their communications protocol development. In doing so, they will then further the NCS's recommendations on device connectivity for multimodal monitoring. 


\title{
Poster 60
}

\section{Comparison of the Efficacy of 4-Factor and 3-Factor Prothrombin Complex Concentrate for Reversing Warfarin in Patients with Intracranial Hemorrhages}

\author{
Lauren H. Peck', Sheri S. Tokumaru ${ }^{1}$, Cherie C. Chu ${ }^{1}$, Kara K. Izumi', Kazuma K. Nakagawa ${ }^{3}$. \\ ${ }^{1}$ University of Hawaii at Hilo/College of Pharmacy, Hilo, HI, USA, ${ }^{2}$ The Queen's Medical Center/Pharmacy \\ Department, Honolulu, HI, USA, ${ }^{3}$ The Queen's Medical Center/Neuroscience Institute, Honolulu, HI, \\ USA.
}

\section{Introduction:}

Warfarin-related intracranial hemorrhage $(\mathrm{ICH})$ is associated with increased mortality due to higher rates of hematoma expansion. Current guidelines recommend rapid anticoagulation reversal using intravenous vitamin $\mathrm{K}$ and prothrombin complex concentrate (PCC). Previous studies show high prevalence of incomplete anticoagulation reversal with 3-factor PCC. We therefore sought to assess the impact of PCC type in reversing warfarin in $\mathrm{ICH}$ patients.

\section{Methods:}

This was a retrospective study of $89 \mathrm{ICH}$ patients (47 traumatic and 42 non-traumatic) with warfarinassociated coagulopathy who were admitted to a level II trauma center between January 2010 and September 2015 and received at least one dose of 3-factor or 4-factor PCC. Post-PCC INR of $£ 1.2$ was considered successful INR reversal. Multivariable model using logistic regression was performed to assess the impact of PCC type on successful INR reversal after adjusting for age, sex, BMI, and baseline INR.

\section{Results:}

Overall, the prevalence of successful INR reversal was $72.2 \%$. There was a higher proportion of patients with successful INR reversal in those who received 4-factor PCC than 3-factor PCC (53.8\% vs. $18.4 \%$, respectively, $p=0.005)$. In the multivariable model, 4-factor PCC (OR 18.1; 95\% Cl: 3.1 to 106.5) and baseline INR (OR per unit of INR 0.4; 95\% Cl: 0.19-0.84) were independent predictors of successful INR reversal. The change in INR post-PCC was significantly greater in those who received 4-factor PCC than 3 -factor PCC (3.60 \pm 4.16 vs. $1.26 \pm 1.11$ respectively, $p<0.0001)$.

\section{Conclusions:}

4-factor PCC more reliably reversed warfarin in ICH patients compared to 3-factor PCC. 


\title{
Poster 61
}

\section{Brain Temperature But Not Core Temperature Increases During Cortical Spreading Depolarizations In Patients With Spontaneous Intracerebral Hemorrhage}

\author{
Mario Kofler ${ }^{1}$, Alois A. Schiefecker ${ }^{1}$, Christian C. Friberg ${ }^{2}$, Ronny R. Beer ${ }^{3}$, Iris I. Unterberger ${ }^{1}$, Bettina B. \\ Pfausler, Chun C. Chun Fai Cheah ${ }^{4}$, Max M. Gaasch', Paul P. Rhomberg ${ }^{5}$, Martin M. Ortler ${ }^{6}$, Claudius C. \\ Thomé $^{6}$, Martin Ejler M. Fabricius ${ }^{2}$, Erich E. Schmutzhard ${ }^{1}$, Raimund R. Helbok ${ }^{1}$.
}

\begin{abstract}
${ }^{1}$ Department of Neurology, Medical University Innsbruck, Innsbruck, Austria, ${ }^{2}$ Department of Clinical Neurophysiology, Rigshospitalet, Copenhagen, Denmark, ${ }^{3}$ Department of Neurology, Medical University Innsbruck, Innsbruck, Austria, ${ }^{4}$ Department of Neurology, Hospital Pulau Pinang and Medical University Innsbruck, Innsbruck, Austria, ${ }^{5}$ Department of Neuroradiology, Medical University Innsbruck, Innsbruck, Austria, ${ }^{6}$ Department of Neurosurgery, Medical University Innsbruck, Innsbruck, Austria.
\end{abstract}

\section{Introduction:}

Cortical spreading depolarizations (CSDs) as highly active metabolic event commonly occur in patients with intracerebral hemorrhage $(\mathrm{ICH})$ and may contribute to secondary brain injury. Fever is an independent predictor for unfavorable outcome after $\mathrm{ICH}$ and may trigger CSDs. Here, we investigated the dynamics of brain-temperature (Tbrain) relative to CSDs and core-temperature (Tcore).

\section{Methods:}

Twenty comatose patients with ICH and multimodal electrocorticograpy (EcoG) monitoring were prospectively enrolled. A subdural EcoG strip was placed adjacent to the evacuated $\mathrm{ICH}$. A combined intracranial pressure (ICP) and Tbrain probe was inserted in the white matter ipsilateral to the ICH. Monitoring data were averaged to 5-minute-means for longitudinal analysis and to one-hour-means. Clusters of CSDs were defined as $\geq 2 \mathrm{CSDs} /$ hour. Fever-burden was defined as $\%$ of temperature $>38.0^{\circ}$ $\mathrm{C}$ per 24-hours. Data were analyzed using GEE-models and are presented as median and interquartile range (IQR).

\section{Results:}

During 3097 hours (173 hours [81-123] /patient) of EcoG monitoring, 342 CSDs were analyzed. 15\% of CSDs occurred in clusters. Baseline Tcore and Tbrain were $37.3^{\circ} \mathrm{C}(36.9-37.8)$ and $37.4^{\circ} \mathrm{C}(36.7-37.9)$, respectively. Tbrain but not Tcore significantly increased 5 minutes preceding the CSDs by a median of $0.2^{\circ} \mathrm{C}(0.1-0.2 ; p<0.001)$ and returned to baseline $35 \mathrm{~min}$ following CSDs. Tbrain $\left(+0.4^{\circ} \mathrm{C}[0.1-0.4]\right.$; $p<0.001)$ but not Tcore $(p=0.34)$ was higher during clusters compared to episodes of single CSDs. CSDs probability was highest during Tbrain $\geq 38.0^{\circ} \mathrm{C}(23 \%$ probability; $n=150)$, compared to $9 \%$ probability at Tbrain $\leq 36.6^{\circ} \mathrm{C}(n=41)$. Fever burden was predictive for the occurrence of CSDs (Tbrain: $p<0.001$; $\mathrm{OR}=1.2$ per \%; Tcore: $\mathrm{p}<0.001 ; \mathrm{OR}=1.1$ per $\%$ ) independent of MAP and ICP.

\section{Conclusions:}

CSDs were triggered during episodes of fever. Our data suggest an association between CSDs and cerebral heat production, especially during clusters. Integration of ECoG monitoring in trials investigating prophylactic normothermia after ICH may help to understand the potential beneficial effect of this intervention. 


\section{Poster 62}

\section{Anticoagulation reversal: Time is of the Essence}

Chandan Mehta ${ }^{1}$, Mathew M. Jones ${ }^{2}$, Mauricio M. Ruiz Cuero ${ }^{1}$, Jody J. Wellwood ${ }^{1}$, Panayiotis P. Mitsias ${ }^{1}$, Mohammed M. Rehman ${ }^{1}$, Panayiotis P. Varelas'.

${ }^{1}$ Henry Ford Hospital, Detroit, MI, USA, ${ }^{2}$ Henry Ford Hospital, Detroit, MI, USA.

\section{Introduction:}

Anticoagulation reversal is recommended for patients with intracerebral hemorrhage (ICH) on vitamin $\mathrm{K}$ antagonists. We propose a window for reversal of Vitamin $\mathrm{K}$ antagonists, in which ICH volume remains below the average growth in a control population on follow up imaging

\section{Methods:}

A retrospective review of Neuro ICU patients at Henry Ford from 2013-2014 was conducted on patients with the ICD9 code for diagnosis of ICH. Inclusion criteria: Brought to ED at onset of symptoms, either not on anticoagulation or were on warfarin with therapeutic INR, and had received PCC administration. Fifty eight total patients were identified. Patients were approximately matched for GCS on admission, ICH volume, age. ICH volumes were independently calculated by two reviewers using the $A B C / 2$ method and were found to have $+/-1 \mathrm{cc}$ difference in volume estimation.

\section{Results:}

Forty-seven control patients were identified: Mean age 67 (44-92), GCS on admission 11 (3-15), SBP $170, \mathrm{ICH}$ volume on admission $20.28 \mathrm{cc}$, ICH volume on repeat scan $23.94 \mathrm{cc}$, change in ICH volume $21.45 \%$, and mean time between initial and stability scans was 1150 minutes. Eleven patients on VKAs were identified who underwent reversal with 4-factor PCC. Mean age 74 (58-88), GCS on admission 11 (3-15), SBP 149, ICH volume on admission $31.81 \mathrm{cc}, \mathrm{ICH}$ volume on repeat scan $42.42 \mathrm{cc}$, and time between scans was 630 minutes. Average INR on presentation was 4.71. All patients on warfarin received Vitamin K. 4/11 patients on warfarin received FFP.

\section{Conclusions:}

Patients who had PCC administered before 195 minutes (7) had a mean change in ICH volume of $16.95 \%$. Patients who had reversal completed after the 195 minutes (4) had a mean change in ICH volume of $60.28 \%$. ( $P$ value $=0.0032$ ). We propose a potential "recommended reversal time" of less than 200 minutes for Vitamin $\mathrm{K}$ antagonists in our institution. Limitations of study include small sample size. 


\section{Poster 63}

\section{Application of the FOUR Score in Intracerebral Hemorrhage Risk Analysis}

Sherri A. Braksick ${ }^{1}$, J. Claude J. Hemphill, Jay J. Mandrekar ${ }^{3}$, Eelco E. Wijdicks ${ }^{4}$, Jennifer J. Fugate ${ }^{4}$.

${ }^{1}$ Department of Neurology, Rochester, MN, USA, ${ }^{2}$ University of California, San Francisco/ Department of Neurology, San Francisco, CA, USA, ${ }^{3}$ Department of Biomedical Statistics and Informatics/ Mayo Clinic, Rochester, MN, USA, ${ }^{4}$ Department of Neurology/ Mayo Clinic, Rochester, MN, USA.

\section{Introduction:}

The Full Outline of UnResponsiveness (FOUR Score) is a validated scale that provides the essentials of a coma examination by incorporating motor response, eye opening and eye movements, brainstem reflexes, and respiratory pattern. We incorporated the FOUR Score into the existing ICH Score and evaluated its accuracy of risk assessment of patients with spontaneous intracerebral hemorrhage (ICH).

\section{Methods:}

Consecutive patients admitted to our institution from 2009-2012 with spontaneous ICH were reviewed. Using patient age, hemorrhage location, hemorrhage volume, evidence of intraventricular extension and GCS, the ICH Score was calculated. The FOUR Score was then incorporated into the ICH Score as a substitution for the GCS (ICH-FOUR Score). The ability of the two scores to predict mortality at 1 month was then compared.

\section{Results:}

In total, 274 patients met inclusion criteria. The median age was 73 years (IQR 60-82) and 138 (50.4\%) were male. Overall mortality at one month was $28.8 \%(n=79)$. The area under the ROC curve was 0.6 $(95 \% \mathrm{Cl} 1.11-2.08)$ for the ICH Score, and $0.6(95 \% \mathrm{Cl} 1.02-1.86)$ for the ICH-FOUR Score. For ICH Scores of 2, 3, 4 and 5, one-month mortality was $18.8 \%, 29.0 \%, 44.7 \%$ and $66.7 \%$. In the ICH-FOUR Score model, mortality was $20.6 \%, 27.8 \%, 39.6 \%$ and $75.0 \%$ for scores of $2,3,4$ and 5 , respectively.

\section{Conclusions:}

The ICH Score and the ICH-FOUR Score predict 1-month mortality with comparable accuracy. As the FOUR Score provides additional clinical information regarding patient status, it may be a reasonable substitute for the GCS into the ICH Score. 


\title{
Poster 64
}

\section{Depression Symptoms Are Associated with Worse Outcomes After Intracerebral Hemorrhage and Are Under-Diagnosed Across Multiple Healthcare Systems}

\author{
Andrew M. Naidech ${ }^{1}$, Brandon B. Francis ${ }^{1}$, Matthew M. Maas ${ }^{1}$, Eric E. Liotta ${ }^{1}$, Jennifer J. Beaumont ${ }^{2}$, \\ Shyam s. Prabhakaran ${ }^{1}$, David D. Cella ${ }^{2}$, Jane J. Holl ${ }^{3}$, Abel A. Kho². \\ ${ }^{1}$ Northwestern University Department of Neurology, Chicago, IL, USA, ${ }^{2}$ Northwestern University \\ Department of Public Health and Medicine, Chicago, IL, USA, ${ }^{3}$ Northwestern University Department of \\ Public Health and Medicine, Chicago, IL, USA.
}

\section{Introduction:}

Depressive symptoms in patients with intracerebral hemorrhage $(\mathrm{ICH})$ are common and are associated with worse outcomes. It is not well described how often depressive symptoms are appropriately ascertained and treated in a multicenter cohort, and whether this is a potential target for improving outcomes.

\section{Methods:}

We retrieved diagnostic codes from four university health systems across Chicago (multicenter cohort). Separately, we prospectively screened for depressive symptoms (NIH Patient Reported Outcomes Measurement Information System, PROMIS, T Score 60), in patients at one prospectively assessed cohort center at one, three and twelve months after ICH onset. We compared detection rates of depressive symptoms between the two samples.

\section{Results:}

Diagnostic codes for depressive symptoms up to three months after ICH onset were recorded in 132 of $3422(3.8 \%)$ of the multicenter cohort versus 26 of $116(22.4 \%)$ in the prospectively screened cohort (OR $7.20,95 \% \mathrm{Cl} 4.5-11.5, \mathrm{p}<0.0001)$. Results were similar considering depressive symptoms up to 12 months after $\mathrm{ICH}, 140$ of 3422 patients in the multicenter $(4.0 \%)$ versus 36 of 116 prospective patients (31.0\%; OR 10.55, 95\% Cl $6.87-16.2, p<0.0001)$. In the multicenter cohort less than $10 \%$ of patients received a selective serotonin reuptake inhibitor (SSRI) within three months of ICH onset.

\section{Conclusions:}

The prevalence of depressive symptoms in survivors of $\mathrm{ICH}$ is more common than would be suggested from diagnostic codes across a metropolitan area. Depressive symptoms after $\mathrm{ICH}$ are likely to be under treated and SSRIs may be an under-utilized therapeutic option. 


\title{
Poster 65
}

\section{The Impact of an Electronic Order Set on Time to Prothrombin Complex Concentrate Administration for Vitamin-K Antagonist-Associated Intracerebral Hemorrhage}

\author{
Jeffrey R. Vitt', Jeffrey J. Vitt ${ }^{1}$, Nirav N. Shah ${ }^{1}$, Lynn L. Do ${ }^{2}$, Anthony A. Kim ${ }^{1}$. \\ ${ }^{1}$ UCSF - Neurology, San Francisco, CA, USA, ${ }^{2}$ UCSF - Pharmacy, San Francisco, CA, USA.
}

\section{Introduction:}

Vitamin $\mathrm{K}$ antagonist (VKA)-associated intracerebral hemorrhages $(\mathrm{ICH})$ are more likely to expand and are associated with higher mortality than primary $\mathrm{ICH}$. Prompt reversal of international normalized ratio (INR) with prothrombin complex concentrate (PCC) may promote hemostasis and decrease hematoma expansion more quickly compared to fresh frozen plasma. The aim of this study was to evaluate the impact of an electronic order set designed to standardize and facilitate more timely reversal of coagulopathy in VKA-associated $\mathrm{ICH}$.

\section{Methods:}

We identified all adults that received PCC for VKA-associated ICH from June 2012 to March 2015 at UCSF Medical Center, which included a period before and after an electronic order set became available in 2014. We abstracted baseline demographics and clinical data from electronic medical records. The primary outcome was time from radiographic identification of $\mathrm{ICH}$ to administration of PCC. Secondary outcomes included PCC dosing accuracy based on actual weight and baseline INR as well as time from PCC order to follow-up INR.

\section{Results:}

We identified 31 patients that received PCC for VKA-associated ICH, including 17 patients before and 14 patients after the order set became available. Baseline demographics and clinical features were similar. Order set use was associated with a significant decrease in the time from identification of $\mathrm{ICH}$ on imaging to the administration of PCC (median 45 vs. 83 minutes; $p=0.02)$, more accurate doses delivered $(92.9 \%$ vs $29.4 \%$; $p<0.01$ ), and a shorter time from the PCC order to follow-up INR (median 85 vs 164 minutes, $p=0.001$ ). Modified Rankin Scale at discharge and in-hospital mortality were similar in the two groups.

\section{Conclusions:}

An electronic order set for administering PCC for VKA-associated ICH was associated with significantly faster time to PCC administration and increased accuracy in dose administered. 


\title{
Poster 66
}

\section{The Spot Sign and Low Platelet Activity Identify Similar Patients at Risk for Hematoma Growth}

\author{
Andrew Naidech, Alan A. Long, Kathryn K. Muldoon, Rajbeer R. Sangha.
}

Northwestern Medicine, Chicago, IL, USA.

\section{Introduction:}

Hematoma growth predicts worse outcomes in patients with intracerebral hemorrhage $(\mathrm{ICH})$, making it crucial to identify patients. Both active contrast extravasation ("spot sign") and lower platelet activity have been associated with hematoma growth. We tested the hypothesis that patients with a spot sign had lower platelet activity.

\section{Methods:}

We prospectively identified patients with acute $\mathrm{ICH}$, measured platelet activity on admission, routinely obtained CT angiography and graded the presence or absence of a spot sign. We limited the analysis to patients who underwent $\mathrm{CT}$ angiography within 12 hours of $\mathrm{ICH}$ symptom onset. Platelet activity was measured with the VerifyNow-ASA (Accumetrics, CA). Non-normally distributed data were compared with the Mann-Whitney test, and binary variables with chi-squared or logistic regression.

\section{Results:}

We studied 166 patients, with mean age $62.7+/-14.2$ years, $51 \%$ were women and $73 \%$ had a history of hypertension. Sixteen $(9.6 \%)$ had a positive spot sign. A spot sign was associated with lower platelet activity (479 [433 - 532] vs. 563 [456 - 637] ARU, $P=0.03$, where <=550 ARU indicates an aspirin effect). Of 16 patients with a spot sign, 13 had platelet activity $<=550$ ARU. Platelet activity $<=550$ ARU was associated with increased odds of a spot sign (OR 5.7, 95\% Cl 1.55-20.7, P=0.009).

\section{Conclusions:}

The presence of a spot sign on CT angiography was associated with lower platelet activity, suggesting a common mechanism. The spot sign and reduced platelet activity both identify patients at higher risk for hematoma growth. 


\title{
Poster 67
}

\section{The Effect of Temperature on Clot Dissolution, and Outcomes, after Spontaneous Intraventricular Hemorrhage: A Retrospective Analysis of the CLEARIII-IVH Data}

\author{
Peter A. Abdelmalik ${ }^{1}$, Rachel R. Dlugash ${ }^{2}$, Nichol N. McBee ${ }^{1}$, Issam I. Awad ${ }^{3}$, Dan D. Hanley ${ }^{1}$, Panayiotis \\ P. Varelas ${ }^{4}$, Wendy W. Ziai'.
}

\footnotetext{
${ }^{1}$ Department of Neurology, The Johns Hopkins University School of Medicine, Baltimore, MD, USA, ${ }^{2}$ Department of Neurology, The Johns Hopkins School of Medicine, Baltimore, MD, USA, ${ }^{3}$ Department of Neurosurgery, University of Chicago, Chicago, IL, USA, ${ }^{4}$ Department of Neurology, Henry Ford Hospital , Detroit, MI, USA.
}

\section{Introduction:}

Introduction: Clot dissolution is a biochemical process catalyzed by enzymatic proteins, requiring a specific temperature range for optimal function. CLEAR III, a randomized, double-blinded, placebocontrolled trial tested whether extraventricular drainage (EVD) plus intraventricular alteplase improved outcome by removing IVH compared to EVD plus saline.

\section{Methods:}

Methods: Retrospective assessment of prospectively collected temperature data q4H over first 7 days post randomization. Blinded assessment of modified Rankin scale (mRS) occurred at 30 and 180 days. Regression and correlation analyses was undertaken to examine the effect of temperature on poor functional outcome (mRS4-6).

\section{Results:}

Results: Median (interquartile range) daily temperature was $37.5(37.4,37.6)^{\circ} \mathrm{C}$ and did not differ between study groups, but peaked later in alteplase treated patients. Both IVH volume $>20 \mathrm{cc}(58 \%)$ and infection during first week (27.8\%) were significantly associated with higher temperature. Patients with mRS 4-6 (vs. 0-3) had significantly higher daily temperatures and weekly average temperature (oC) (median, [IQR]) at 30 days [37.42 $(37.11,37.72)$ vs. $37.20(36.98,37.43), p 37.5^{\circ} \mathrm{C}$ was significantly associated with faster IVH clot lysis rate (Spearmans rho $0.12 ; p=0.006$ ), but despite significantly higher temperatures, patients with mRS4-6 (vs. mRS0-3) had significantly lower average percentage IVH removal at day 30 $(51.23 \pm 45.77 \%$ vs. $63.06 \pm 28.72 \%, p<0.001)$ and $180(48.82 \pm 51.47 \%$ vs. $58.34 \pm 31.02 \%, p=0.008)$. Temperature burden $\geq 37.5^{\circ} \mathrm{C}$ over first seven days was associated with increased odds ratio for poor functional outcome of 1.91 (95\% Cl:1.45-2.52) and $1.54(95 \% \mathrm{Cl}: 1.26-1.90)$ at 30 and 180 days respectively, and was an independent predictor of poor functional outcome at day 30, but not 180 after adjustment for early infection and other severity predictors.

\section{Conclusions:}

Conclusion: Temperatures $\geq 37.5^{\circ} \mathrm{C}$ recorded over the first seven days after spontaneous IVH are associated with faster clot lysis rate, but also with poor functional outcome. Negative associations with higher temperature likely outweigh enhanced clot resolution. 


\section{Poster 68}

\section{Prothrombin Complex Concentrates in Warfarin-Associated Intracerebral Hemorrhage: Are We Underdosing?}

Jamil Dibu.

Neurological Institute, Cleveland Clinic, Cleveland, OH, USA.

\section{Introduction:}

Prothrombin complex concentrates (PCC) are recommended for coagulopathy reversal in warfarinassociated intracerebral hemorrhage (WAICH). However, the percentage of patients who achieve target reversal for different INR ranges is not well characterized over time, and the appropriate dose for INR 1.4 -1.9 is not known.

\section{Methods:}

A prospective study of patients with spontaneous WAICH was conducted from 5/22/13-12/29/15. Patients on warfarin received vitamin $\mathrm{K}(10 \mathrm{mg}$ IV) and PCC (Kcentra) dosed at $10 \mathrm{u} / \mathrm{kg}$ for INR 1.4-1.9, $25 \mathrm{u} / \mathrm{kg}$ for INR 2.0-3.9, $35 \mathrm{u} / \mathrm{kg}$ for INR 4.0-6.0 and $50 \mathrm{u} / \mathrm{kg}$ for INR>6.0. Repeat INRs were checked $30 \mathrm{~min}$. after dosing and then every $6 \mathrm{~h}$ for $24 \mathrm{~h}$. Target INR reversal was defined as INR $<1.4$. Thrombotic and hemorrhagic complications during hospitalization were recorded.

\section{Results:}

Of $162 \mathrm{ICH}$ patients, 26 patients (16\%) had WAICH and received Kcentra. The INR ranges were: 1.4-1.9 in 5 patients (19\%), 2.0-3.9 in $17(65 \%), 4.0-6.0$ in $1(4 \%)$ and $>6.0$ in 3 patients $(12 \%)$. Within 30 minutes of PCC administration INR<1.4 occurred in 2/5 (40\%), 14/17 (82\%), 1/1 (100\%) and 2/3 (67\%), in each INR group, respectively. Three patients with INR 1.9 received $10 \mathrm{u} / \mathrm{kg}$ of PCC, while 2 with INR 1.9 received $25 \mathrm{u} / \mathrm{kg}$ and the 30-minute repeat INR ranges were 1.4-1.5 and 1.0-1.1, respectively. FFP was administered to $1(20 \%), 3(18 \%), 0(0 \%)$ and $1(33 \%)$ patient in each group for a persistently elevated INR. At $6 \mathrm{~h}$ post-PCC, INR reversal occurred in 100\%, 69\% (rest had INR 1.4), 100\%, and 67\%, respectively. At 24h, 25/26 (96\%) patients had INR $<1.4$. Two patients had venous thromboembolisms and one had DIC following PCC administration.

\section{Conclusions:}

In this small case series of WAICH patients, INR correction with Kcentra was adequate except for those with INR 1.4-1.9, where a higher dose $(25 \mathrm{u} / \mathrm{kg})$ provided better INR correction. 


\title{
Poster 69
}

\section{Prevalence of stroke following craniotomy or craniectomy for spontaneously intracranial hemorrhage}

\author{
Nasim Ahmed ${ }^{1}$, yaniv y. Fenig ${ }^{2}$, Joshua J. Trostinksky ${ }^{3}$. \\ ${ }^{1}$ Jersey Shore University Medical Center, Division of Trauma \& Neuro Critical Care, Neptune, NJ, USA, \\ ${ }^{2}$ Monmouth Medical Center, long Branch, NJ, USA, ${ }^{3}$ Jackson Liberty Highschool, Jackson, NJ, USA.
}

\section{Introduction:}

The purpose of the study was to evaluate the prevalence of stroke after all cranial operations (craniotomy and craniectomy) following spontanous intrac-cranial hemorrhage $(\mathrm{SICH})$

\section{Methods:}

Data was extracted from the American college of Surgeon National surgical Quality Improvement program (ACS NISQIP) from 2005 through 2014. All adult patients who underwent craniotomy or craniectomy for $\mathrm{SICH}$ were included in the study. SICH was identifying by the International Classification Disease, Ninth Revision, and Clinical Modification (ICD-9-CM) code 431. Patient demography and clinical information was extracted and compared between the patients who developed stroke and who did not as a part of complication. Age was categorized at 65 years. Morbid obesity was defined if the body mass index was 35 or above. Data was presented with proportion. Chi square test was performed to compare the two groups. Multiple logistic regression models was used to identify any risk factors if the $P$ value was $\leq 0.5$ in bivariate analysis. $P$ values $\leq 0.05$ was considered statistically significant.

\section{Results:}

Of 2640 patients, 607 patients underwent craniotomy or craniectomy for $\mathrm{SICH}$. The stroke was presented as a postoperative complication in 14/607 (2.3\%) patients. There were no significant differences between the groups (stroke positive versus stroke negative ) regarding age 65 or above ( $21.43 \%$ versus $40.81 \%$, $P=0.14)$, male gender ( $35.7 \%$ versus $52.28, P=0.22)$, histories of smoking $(14.29 \%$ versus 18.38 , $\mathrm{P}=0.69)$, of hypertension (71.43 versus $60.03 \%, \mathrm{P}=0.38)$, of Cerebral vascular accident (CVA) $(21.43 \%$ versus $24.11 \%, P=0.81)$, of diabetes $(21.43 \%$ versus $15.85 \%, P=0.57)$, of morbid obesity $(21.43 \%$ versus $14.33 \%, \mathrm{P}=0.45)$ and evacuation of intracranial hematoma $(78.57 \%$ versus $81.96 \%, \mathrm{P}=0.74)$ respectively. Multiple logistic regression models did not show any significant association of patient's characteristics and development of post-operative stroke prevalence.

\section{Conclusions:}

Study showed the prevalence of postoperative stroke following craniotomy or craniectomy for spontaneous intracranial hemorrhage was around $2.3 \%$. 


\title{
Poster 70
}

\section{Neuroscience Intensive Care Unit Non-Admission Criteria for Patients with Primary Intracerebral Hemorrhage}

\author{
Ayham M. Alkhachroum ${ }^{1}$, Oladi O. Bentho ${ }^{2}$, Ashish A. Kulhari ${ }^{3}$, Neel N. Chari ${ }^{4}$, Wei W. Xiong ${ }^{3}$. \\ ${ }^{1}$ University Hospitals Case Medical Center Neurology Department, Cleveland, OH, USA, ${ }^{2}$ Johns Hopkins \\ University Neurocritical Care, Baltimore, MD, USA, University Hospitals Case Medical Center, Cleveland, \\ $\mathrm{OH}, \mathrm{USA},{ }^{4}$ Case Western Reserve University School of Medicine, Cleveland, OH, USA.
}

\section{Introduction:}

Intensive care resources, particularly, neuroscience intensive care resources are limited and costly. In most institutions in the country, all intracerebral hemorrhage $(\mathrm{ICH})$ patients are admitted to the Neuroscience intensive care unit. We sought to identify what criteria will allow us to determine which primary intracerebral hemorrhage patients will not need admission to an intensive care unit (ICU).

\section{Methods:}

We studied retrospectively 423 patients with primary ICH from January 2013 to the end of Dec 2015. We reviewed multiple admitting characteristics: demographics, hematoma volume, location of hemorrhage, any brain compression, blood pressure, respiratory status, INR, Glasgow Coma Score (GCS). The reasons for neuroscience intensive care unit admission requirements are the need for mechanical ventilation, hydrocephalus, increased intracranial pressure, Low GCS score, hematoma expansion, or secondary ICH (aneurysm, AVM, trauma, AC). We looked at the patients who had short length of stay in the unit, and who did not require any ICU care intervention. This group had the following characteristics: Supratentorial $\mathrm{ICH}, \mathrm{ICH}$ volume 12. We called this criteria the "Non-admission Criteria for Patients with Primary Intracerebral Hemorrhage".

\section{Results:}

423 patients were identified as primary ICH. 69 patients (16.3\%) fulfilled the non-admission criteria to the neuro-intensive care unit. Of 69 patients 27 patients (39.1\%) discharged home, 31 patients to acute rehab facility $(44.9 \%), 10$ patients discharged to SNF (14.5\%), 1 patient died $(1.5 \%)$; elderly patient with existing DNR comfort care orders. None of the 69 patients had to be readmitted to neuroscience intensive care unit, and none required neurosurgical procedure.

\section{Conclusions:}

We propose that $\mathrm{ICH}$ patients that fulfill the non-admission criteria do not have to be admitted to an ICU and can safely be monitored in a step down unit This represented about $16 \%$ of ICH patients at our tertiary academic medical center. Future prospective studies are required to validate the criteria. 


\section{Poster 71}

\section{Evaluation of a Simplified 4-Factor Prothrombin Complex Concentrate Dosing Protocol for the Reversal of Warfarin-Related Intracranial Hemorrhage Patients}

Joseph R. Blunck, Justin J. Shewmaker.

Saint Lukes Hospital / Pharmacy, Kansas City, MO, USA.

\section{Introduction:}

Current guidelines recommend the use of 4 -factor prothrombin complex concentrate (PCC) rather than fresh frozen plasma for reversal of warfarin-related intracranial hemorrhage (WRICH). There is no consensus regarding an optimal PCC dosing strategy, but limited data suggest that PCC dosing should be based on weight and international normalized ratio (INR). In November of 2013, our health system implemented a WRICH reversal protocol with a 4-factor PCC dosing nomogram that utilized fixed-dose options of 2000, 2500, 3000 or 3500 units based on weight above or below 80 kilograms and initial INR above or below 4 . The purpose of this study is to evaluate the effectiveness and safety of this simplified 4-factor PCC dosing protocol.

\section{Methods:}

Patients given 4-factor PCC for WRICH reversal in our health system were retrospectively identified with billing codes. Chart review was completed to evaluate the primary endpoint of achieving a post treatment goal INR of $\leq 1.4$. Secondary endpoints include evidence of a venous thromboembolic event, time to goal INR, difference from package insert (PI) dosing, discharge Modified Rankin Scale, and in-hospital mortality.

\section{Results:}

We identified 82 WRICH patients from November 2013 through April 2016 that were reversed with our simplified 4-factor PCC dosing protocol. Seventy-seven (93.9\%) patients achieved a post reversal goal INR $\leq 1.4$ with a mean (SD) baseline INR of $2.8(1.6)$ and post treatment INR of $1.2(0.1)$. Fifty-six $(68.3 \%)$ patients received a PCC dose equivalent to rounded PI dosing and the mean (SD) dose difference between groups was 2321 (365) units vs. 2256 (528) units, protocol vs. PI dosing, respectively. One patient (1.2\%) had a thromboembolic event that occurred 20 days post reversal.

\section{Conclusions:}

A simplified 4-factor PCC dosing protocol is a safe and effective strategy for WRICH reversal. 


\title{
Poster 72
}

\section{The Utility of Charlson Comorbidity Index in Predicting Outcomes in Patients with Primary Intracerebral Hemorrhage}

\author{
Ayaz M. Khawaja ${ }^{1}$, Amelia A. Boehme ${ }^{2}$, Karen K. Albright ${ }^{3}$, Kumar K. Gyanendra ${ }^{4}$, Michael M. Lyerly ${ }^{3}$, \\ Anand A. Venkatraman ${ }^{3}$, Maira M. Mirza ${ }^{5}$, Mark M. Harrigan ${ }^{6}$.
}

${ }^{1}$ University of Alabama at Birmingham Hospital/Neurology, Birmingham, AL, USA, ${ }^{2}$ Gertrude $H$. Sergievsky Center, Columbia University / Neurology, New York, NY, USA, ${ }^{3}$ University of Alabama at Birmingham Hospital / Neurology, Birmingham, AL, USA, ${ }^{4}$ Mayo Clinic Arizona / Neurology, Phoenix, AZ, USA, ${ }^{5}$ Sinai-Grace Hospital, Detroit Medical Center / Internal Medicine, Detroit, MI, USA, ${ }^{6}$ University of Alabama at Birmingham Hospital / Neurosurgery, Birmingham, AL, USA.

\section{Introduction:}

The Charlson Comorbidity Index $(\mathrm{CCl})$ is used to estimate risk of in-hospital, 1 year and 10 year mortality, and has been validated in patients with acute ischemic stroke. Previous studies have shown that $\mathrm{CCl}$ influences functional outcomes. We sought to evaluate the association between $\mathrm{CCl}$ and outcomes in patients with primary intracerebral hemorrhage $(\mathrm{ICH})$.

\section{Methods:}

Patients admitted to our center with primary-ICH from 2008-2013 were included. Demographic and clinical data were collected. The primary outcome measures were the proportion of patients with discharge mRS (dmRS) of 4-6, death and poor discharge disposition (any disposition other than home or inpatient-rehabilitation). Crude and adjusted logistic regression were used to evaluate the association between $\mathrm{CCl}$ and outcomes.

\section{Results:}

383 patients were identified. There were $37(9.7 \%)$ patients with a CCl of 0 or $1,242(63.2 \%)$ patients with a CCl of 2-5, and $104(27.2 \%)$ with a CCl of 6 or greater. While the continuous CCl was not significantly associated with a dmRS of $4-6$ (OR $1.08,95 \% \mathrm{Cl} 0.99-1.19, \mathrm{p}=0.09)$, it was associated with disposition. The odds of poor disposition increased $18 \%$ with each increase in $\mathrm{CCl}$ (OR $1.18,95 \% \mathrm{Cl}$ $1.08-1.28, p=0.0003)$. The odds of death increases $12 \%$ with each point increase in $\mathrm{CCl}(\mathrm{OR} 1.12,95 \%$ $\mathrm{Cl} 1.02-1.23, \mathrm{p}=0.021)$. After adjusting for baseline $\mathrm{ICH}$ score, $\mathrm{CCl}$ remains significantly associated with poor disposition (OR 1.13, 95\% $\mathrm{Cl} 1.00-1.27, \mathrm{p}=0.042$ ), however the association between $\mathrm{CCl}$ and death was not statistically significant (OR $1.05,95 \% \mathrm{Cl} 0.91-1.21, \mathrm{p}=0.52)$.

\section{Conclusions:}

In contrast to previous studies, $\mathrm{CCl}$ was not associated with poor short-term functional outcome or inhospital mortality in $\mathrm{ICH}$ patients after adjusting for $\mathrm{ICH}$ score. However, it was significantly associated with poor discharge disposition. This suggests that cumulative comorbidities only predict disposition in $\mathrm{ICH}$, because the $\mathrm{ICH}$ score strongly impacts poor functional outcome and in-hospital mortality. 


\title{
Poster 73
}

\section{Enhanced Renal Clearance in Patients with Intracerebral Hemorrhage}

\author{
Kathryn A. Morbitzer ${ }^{1}$, J. Decrick J. Jordan ${ }^{2}$, Kelly K. Sullivan ${ }^{3}$, Emily E. Durr ${ }^{3}$, Casey C. Olm-Shipman ${ }^{2}$,
} Denise D. Rhoney ${ }^{1}$.

${ }^{1}$ UNC Eshelman School of Pharmacy, Chapel Hill, NC, USA, ${ }^{2}$ UNC School of Medicine, Department of Neurology, Chapel Hill, NC, USA, ${ }^{3}$ UNC Hospitals, Department of Pharmacy, Chapel Hill, NC, USA.

\section{Introduction:}

Accurate assessment of renal function remains a unique challenge in patients with intracerebral hemorrhage $(\mathrm{ICH})$. Mathematical estimates of creatinine clearance $(\mathrm{CrCl})$ routinely used are often inaccurate in this setting. Subsets of critically ill patients have been shown to exhibit a hyperdynamic response leading to an enhanced renal clearance. No studies exist evaluating the directly measured creatinine clearance of patients with $\mathrm{ICH}$.

\section{Methods:}

This was a single-center prospective observational study of adult patients with ICH admitted to the NSICU between January 2015 and July 2015. Eight-hour urinary creatinine clearances were performed daily to directly measure $\mathrm{CrCl}$ until the patient no longer had a foley catheter or the patient left the NSICU. Urinary $\mathrm{CrCl}$ were compared with routine estimated $\mathrm{CrCl}$ based on the Cockcroft-Gault equation. Statistical significance was defined as $p$-value $<0.05$.

\section{Results:}

Thirty patients with $\mathrm{ICH}$ were enrolled in the study. The study sample was $60 \%$ male with a mean age of $70 \pm 13.7$ years. The median admission ICH score was 3 (IQR $2-4$ ) with a mean ICH volume of $64 \pm 64.1$ $\mathrm{mL}$. The median admission GCS was 7.5 (IQR $5-13$ ) and median admission SOFA score was 4.5 (IQR 2 -5). The mean urinary $\mathrm{CrCl}$ over the study period was $119.5 \pm 57.2 \mathrm{~mL} / \mathrm{min} / 1.73 \mathrm{~m} 2$. This differed significantly from the estimated $\mathrm{CrCl}$ over the study period of $77.8 \pm 27.7 \mathrm{~mL} / \mathrm{min} / 1.73 \mathrm{~m} 2(p<0.05)$. Additionally, the mean urinary $\mathrm{CrCl}$ was significantly higher than the estimated $\mathrm{CrCl}$ each individual study day with a mean maximum urinary $\mathrm{CrCl}$ of $129.6 \mathrm{~mL} / \mathrm{min} / 1.73 \mathrm{~m} 2$ on Day 4 .

\section{Conclusions:}

Patients with $\mathrm{ICH}$ consistently experienced urinary $\mathrm{CrCl}$ greater than estimated $\mathrm{CrCl}$ predicted based on the Cockcroft-Gault equation. As renally eliminated medications are routinely dosed based on mathematical estimates of renal function, further study is needed to optimize medication regimens in this patient population to prevent underexposure. 


\title{
Poster 74
}

\section{Novel quantitative imaging markers for understanding cognitive reserve in subdural hematoma (SDH) patients}

\author{
Neha S. Dangayach ${ }^{1}$, Kelly K. Nicol ${ }^{2}$, Stanislaw S. Sobotka ${ }^{3}$, Stephen S. Griffiths ${ }^{4}$, Anthony A. Costa ${ }^{3}$, \\ James J. Lee ${ }^{3}$, Stephan S. Mayer, Jennifer J. Frontera ${ }^{5}$, Errol E. Gordon ${ }^{1}$, Joshua J. Bederson ${ }^{3}$.
}

${ }^{1}$ Mount Sinai Health System/Neurosurgery and Neurology, New York, NY, USA, ${ }^{2}$ Icahn School of Medicine at Mount Sinai/Neurosurgery, New York, NY, USA, ${ }^{3}$ Mount Sinai Health system/Neurosurgery, New York, NY, USA, ${ }^{4}$ Mount Sinai Health System/Institute of Critical Care Medicine, New York, NY, USA, ${ }^{5}$ Cleveland Clinic Foundation/Neurology, Cleveland, OH, USA.

\section{Introduction:}

The Cognitive reserve hypothesis suggests that variations in patient pre-morbid status such as education, occupation and brain morphology influence outcome. This has been extensively validated in patients with Alzheimer's disease. An important component of Cognitive reserve is brain morphology, which can be quantified with measures such as whole brain- or gray matter volume. This study examines use of novel measures of brain morphology to measure cognitive reserve in patients with SDH.

\section{Methods:}

56 patients with CT-documented SDH were screened. We identified patients who had a clinically indicated post-morbid MRI, Telephone Interview for Cognitive Status (TICS) and Barthel Index (BI) at discharge and follow-up. MRI was used to measure several volumes such as whole brain-, gray matter-, left vs right caudate-, hippocampal and intraventricular CSF volume using a Freesurfer pipeline. Primary outcome measures were TICS at 3 and $\mathrm{BI}$ at 12 months

\section{Results:}

7/56 patients with SDH underwent an MRI, TICS and BI assessment at discharge and follow-up. Regional brain volumes were determined, taking 10 hours per patient. Brain volume was $951 \mathrm{cc}(+/-213)$ (SD), intracranial volume was $1426 \mathrm{cc}(+/-226)$, left caudate volume was $2.83+/-0.7$, CSF volume was $1.6+/-$ 0.8. TICS was $26.1+/-9$ at discharge and $30.8+/-8.3$ at follow-up (3-12 months), BI was $95(+/-6.4)$ at 12 mo. We found a positive correlation between intraventricular CSF volume and $\mathrm{BI}$ at $12 \mathrm{mo}(r=0.77$, $\mathrm{p}=0.04$ ). There was also a significant correlation between left caudate volume and $\mathrm{BI}$ at $12 \mathrm{months}$. Brain morphology did not correlate with TICS outcome at 3 months.

\section{Conclusions:}

Quantitative imaging can be used to predict functional outcomes in patients with intracranial hemorrhages. As we continue enrollment we hope to generate meaningful novel markers of cognitive reserve and recovery in patients with intracranial hemorrhages and other pathologies like acute stroke and brain tumors. 


\section{Poster 75}

\section{Predictors of Surgical Intervention for Intracerebral Hemorrhage at a High Volume Tertiary Medical Center}

Christian Hernandez, Vivien V. Lee, Bichun B. Ouyang, Torrey T. Birch.

Rush University Medical Center/Department of Neurological Sciences, Chicago, IL, USA.

\section{Introduction:}

It remains unclear which patients with intracerebral hemorrhage $(\mathrm{ICH})$ benefit from surgical hematoma evacuation, and the patient factors associated with the decision to pursue surgical intervention are largely unknown. We sought to determine predictors of early surgical intervention for $\mathrm{ICH}$ and to investigate if an age bias exists at our institution.

\section{Methods:}

One hundred and twenty consecutive patients who were admitted to the Neurosciences Intensive Care Unit with primary ICH between April 2010 and January 2012 were retrospectively reviewed. Multivariate regression analysis was used to analyze if select patient factors were associated with the decision to pursue early surgical intervention.

\section{Results:}

In total 120 patients were analyzed; 60 patients (50\%) were female (mean age $65.6 \pm 13.3$ ), 104 patients $(87 \%)$ had supratentorial $\mathrm{ICH}$, and 17 patients $(14 \%)$ underwent hematoma evacuation. In univariate analysis age, race, and gender were not significantly associated with the likelihood of surgical intervention. The only factors associated with hematoma evacuation were GCS score and ICH volume. After controlling for significant variables, multivariate analysis showed that the only factor associated with surgical intervention was ICH volume (OR 24.74, Cl 4.59-133.47, $\mathrm{p}=0.0002)$. Surgical intervention did not affect discharge disposition ( $p=0.31$ ), but was associated with a longer length of stay ( 14 vs 6 days, $\mathrm{p}=0.001$ ).

\section{Conclusions:}

In this analysis, $\mathrm{ICH}$ volume was the only predictor of hematoma evacuation in patients with primary ICH. Age and sex did not influence patient selection for surgical intervention. Surgical treatment did not affect patient disposition at discharge, but was associated with longer hospitalizations. Further investigation is needed to determine which patients with $\mathrm{ICH}$ benefit most from early hematoma evacuation. 


\section{Poster 76}

\section{The Effect of Admission Systolic Blood Pressure on Mortality in Patients with Acute Ischemic Stroke}

Ayaz M. Khawaja', Amelia A. Boehme ${ }^{2}$, Harn H. Shiue ${ }^{3}$, April A. Sisson ${ }^{1}$, Michael M. Lyerly ${ }^{1}$, Anand A. Venkatraman ${ }^{1}$, Maira M. Mirza ${ }^{4}$, Toby T. Gropen ${ }^{1}$.

${ }^{1}$ University of Alabama at Birmingham Hospital / Neurology, Birmingham, AL, USA, ${ }^{2}$ Gertrude H. Sergievsky Center, Columbia University / Neurology, New York City, NY, USA, ${ }^{3}$ University of Alabama at Birmingham Hospital / Pharmacy, Birmingham, AL, USA, ${ }^{4}$ Sinai-Grace Hospital, Detroit Medical Center / Internal Medicine, Detroit, MI, USA.

\section{Introduction:}

The effect of initial systolic blood pressure on acute ischemic stroke (AIS) outcomes has been poorly studied. We investigate factors associated with admission systolic blood pressure (SBP), including hemorrhagic transformation (HT) and discharge outcome.

\section{Methods:}

This is a retrospective study of consecutive AIS patients presenting from April 2014 to March 2015. Demographic and clinical data were collected. Admission SBP was divided into three tiers: 165 . The primary outcome measure was in-hospital mortality.

\section{Results:}

Seven Hundred Seventy Six patients were included (mean-age $64 ; 49.2 \%$ female; $36.5 \%$ Black). There were 287 patients with SBP>165 (40.0\%), 251 with SBP 140-165 (32.3\%), and 238 with SBP165 were Black $(43.0 \% ; p=0.0255)$ and had a history of hypertension $(87.1 \% ; p 165$. Premorbid use of antihypertensives did not differ among the three groups. There were no differences in proportion of HT $(15.7 \%$ vs. $18.7 \%$ vs. $12.6 \%$; $p=0.1462)$. Although patients treated with IV-tPA were evenly distributed among tiers $(14.4 \%$ vs. $13.6 \%$ vs. $15.7 \%)$, more patients with SBP165 had mRS 3-6 $(p=0.0382)$. Compared to SBP165 was associated with lower odds of in-hospital mortality $(\mathrm{OR} 0.536,95 \% \mathrm{Cl}=0.295$ $0.975, p=0.041)$. This remained significant after adjusting for age and NIHSS (OR $0.431,95 \% \mathrm{Cl} 0.193-$ $0.962, p=0.0399)$.

\section{Conclusions:}

Normal presenting SBP $(<140)$ in patients with AIS was associated with worse discharge functionaloutcome and higher in-hospital mortality. These differences may be related to other associated medical conditions such as pre-existing heart failure. Further research is needed to define the ideal range to maintain SBP after AIS. 


\title{
Poster 77
}

\section{Does NIH Stroke Scale Predict Length Of Stay (LOS) In Patients With Intracranial Bleed (ICB)?}

\author{
Johnnie Dillinger ${ }^{1}$, Gnan G. Thakore ${ }^{2}$, Katz K. Lisa ${ }^{3}$, Juan J. Gomez ${ }^{4}$, Ronald R. Markert ${ }^{4}$. \\ ${ }^{1}$ Premier Health Partners/Medicine, Dayton, OH, USA, ${ }^{2}$ Neurocritical Care, Dayton, OH, USA, ${ }^{3}$ \\ Neurosurgery, Dayton, OH, USA, ${ }^{4}$ Medicine, Dayton, OH, USA.
}

\section{Introduction:}

NIH Stroke Scale (NIHSS) is a widely used measure of deficits in Intracranial Bleed (ICB). Deficits dictate discharge plans. Majority deficits manifest by 48 hours. Is NIHSS at 48 hours (NIHSSd2) helpful for ICB Length of stay (LOS) and discharge evaluation? Since LOS is important and complex issue, does NIHSSd2 help identify challenging patients?

\section{Methods:}

Between April 2009 and February 2011, 237 patients with non-traumatic, non-aneurysmal and nonmalignant parenchymal cerebral ICB were identified. The associations of NIHSS at presentation (NIHSSp), NIHSS at 48 hours (NIHSSd2), size of ICB, comorbidities, and infection with LOS were investigated retrospectively.

\section{Results:}

The mean age for the 237 patients was $68.7 \pm 13.8$ years and $52 \%$ were male while most patients were white $(68 \%)$. The most prevalent comorbidity was hypertension $(83 \%)$. The mean bleed size was $18.2 \pm 22.7$ cc. Fourteen percent of patients had neurosurgical intervention, $35 \%$ of patient acquired infection during the hospital stay while $40 \%$ required a ventilator. The mean NIHSSp was 14.8 \pm 11.6 , and the mean NIHSSd2 was 15.0 $\pm 12.1(n=229)$. The mean LOS was $11.8 \pm 10.7$ days. NIHSSp and LOS were correlated at $0.10(p=0.14)$ while the correlation for NIHSSd2 and LOS was $0.22(p=0.001)$. LOS initially increased and subsequently decreased with higher NIHSSd2: Patients going Home/Rehab had shortest LOS (8.05 \pm 4.50$)$ and lowest NIHSSd2 (6.00 \pm 6.29$)$; those discharged to Nursing/LTACH had a longer LOS $(20.80 \pm 13.34)$ with a higher NIHSSd2 (17.43 \pm 9.54$)$. Patients experiencing Hospice/Death had lower LOS $(9.26 \pm 9.60)$ and highest NIHSSd2 (27.85 \pm 8.72$)$. Patients with infection were more likely to have an LOS of six days or longer $(96.3 \%)$ vs. without infection $(59.4 \%),(p<0.001)$.

\section{Conclusions:}

NIHSSd2 is a useful measure of LOS and should be collected for patients with ICB. NIHSSd2 at upper teens present most challenges to discharge and should be the aggressive focus for discharge planners. Incidence of infection and ventilator need is high in this population, adding to the challenges. 


\title{
Poster 78
}

\section{Treatment Patterns and Outcomes of Patients Receiving Oral Factor Xa Inhibitors or Low Molecular Weight Heparin who Experienced Life-threatening Intracranial Hemorrhage: A Retrospective Chart Review}

\author{
Truman J. Milling ${ }^{1}$, Charles C. Feronti ${ }^{2}$, Carol C. Clark ${ }^{3}$, Gregory G. Fermann ${ }^{4}$, Shlee S. Song ${ }^{5}$, Sam S. \\ Torbati ${ }^{5}$, Jeffrey J. Weiss ${ }^{6}$, Alexandros A. Charitou ${ }^{7}$, W Richey W. Newman ${ }^{8}$.
}

\footnotetext{
${ }^{1}$ Seton Dell Medical School Stroke Institute, Austin, TX, USA, ${ }^{2}$ Allegheny General Hospital, Pittsburgh, PA, USA, ${ }^{3}$ Beaumont Hospital-Royal Oak, Royal Oak, MI, USA, ${ }^{4}$ University of Cincinnati College of Medicine, Cincinnati, OH, USA, ${ }^{5}$ Cedar-Sinai Medical Center, Los Angeles, CA, USA, ${ }^{6}$ Navigant Consulting, London, United Kingdom (Great Britain), ${ }^{7} A N X$ Medical Consulting, London, United Kingdom (Great Britain), ${ }^{8}$ Portola Pharmaceuticals, South San Francisco, CA, USA.
}

\section{Introduction:}

Although oral Factor Xa inhibitors (FXal), used for the prevention and treatment of venous thromboembolism and stroke, show lower rates of intracranial hemorrhage (ICH) than warfarin in clinical studies, intracranial bleeding is still associated with high morbidity and mortality. Moreover, there are no specific guidelines for managing these bleeds other than empirical institution-based hemorrhage protocols. There is a need to understand the real-world management, outcomes, and resource utilization of FXal-associated major bleeding in order to potentially improve morbidity and mortality in these patients.

\section{Methods:}

Five US medical centers participated in a retrospective study of patients admitted to the hospital with lifethreatening bleeding on or after January 2014 while on apixaban, rivaroxaban, or low molecular weight heparin $(\mathrm{LMWH})$. Baseline characteristics, treatment patterns, outcomes, and resource utilization were assessed. ICH patients were reviewed and are reported specifically.

\section{Results:}

This interim report includes 33 major bleed patients, including $8 \mathrm{ICH}$. The majority of ICH were hypertensive [7(88\%)], 3(38\%) were on concomitant anti-platelet with FXal, 2(25\%) were diabetic and 2 $(25 \%)$ had a prior history of stroke. The known cause of the ICH was spontaneous $(57 \%)$ and trauma $(43 \%)$. In the management of ICH bleeding, $50 \%$ of patients received clotting factors (e.g. PCC), whereas $25 \%$ received interventions (e.g. radiological embolization). Within 30 days of discharge, 5 (63\%) died, 4 of which occurred during the admission. For the 3 patients who did not die, length of hospitalization was 3.5(3.1-47.0) days, with only 1 discharged patient restarting on an anticoagulant.

\section{Conclusions:}

This study provides a real-world picture of patients receiving FXals or LMWH, experiencing lifethreatening intracranial bleeds. Despite efforts to restore hemostasis, mortality remains high and substantial healthcare resources are expended. This highlights the need to develop specific strategies for management of $\mathrm{ICH}$ in patients who taking FXals and $\mathrm{LMWH}$. 


\title{
Poster 79
}

\section{Disparities in the Use of Seizure Medications for Patients with Intracerebral Hemorrhage}

\author{
Andrew Naidech ${ }^{1}$, Brandon B. Francis ${ }^{1}$, Kathryn K. Muldoon ${ }^{2}$, Matthew M. Maas ${ }^{1}$, Eric E. Liotta ${ }^{1}$, Jane J. \\ Holl ${ }^{1}$. \\ ${ }^{1}$ Northwestern Medicine, Chicago, IL, USA, ${ }^{2}$ Northwestern Medicine, Chicgao, IL, USA.
}

\section{Introduction:}

Prophylactic seizure medications are not recommended by guidelines for patients with intracerebral hemorrhage, yet are prescribed to nearly $40 \%$ of them. There are few data on disparities in their use. We tested the hypothesis that there are differences in the administration of seizure medication, specifically levetiracetam, by patient characteristics and racial/ethnic groups.

\section{Methods:}

We electronically retrieved information from patients who were diagnosed with intracerebral hemorrhage from HealthLNK, a multi-center electronic repository in Chicago, IL, from 2006-2012 ("multicenter cohort"). From 2007 through 2015, we prospectively identified patients with intracerebral hemorrhage at one site ("prospective cohort").

\section{Results:}

There were 3,422 patients in the multicenter cohort from four sites. The use of levetiracetam varied with race/ethnicity $(\mathrm{P}=0.0000008)$, with Caucasians more likely to be administered levetiracetam than AfricanAmericans (OR $1.71,95 \% \mathrm{Cl} 1.43-2.05, \mathrm{P}<0.0001)$. In the prospective cohort $(\mathrm{N}=450)$, hematoma location, older age, depressed consciousness, larger hematoma volume and no alcohol abuse were associated with levetiracetam administration $(P<=0.04$ for all). Caucasians were more likely than AfricanAmericans to receive levetiracetam (OR 1.9, 95\% Cl 1.25 - 2.89, $\mathrm{P}=0.002$ ). African-Americans were more likely to have historical hypertension, higher blood pressure and deep hematomas. Only hematoma location was independently associated with levetiracetam administration $(P<0.00001)$

\section{Conclusions:}

Seizure medication use is predominantly driven by lobar hematoma location. There are racial/ethnic disparities in the use of levetiracetam and they are confounded by hematoma location, a datapoint not typically available in administrative datasets. Deviation from guidelines for the use of seizure medications is common and rational, and any impact on outcomes is uncertain 


\title{
Poster 80
}

\section{Greater gains in late recovery for intracerebral hemorrhage patients with more debilitating initial injury.}

\author{
Anirudh Sreekrishnan ${ }^{1}$, Audrey A. Leasure ${ }^{1}$, Sonya S. Zhou', David D. Hwang ${ }^{1}$, David D. Greer ${ }^{1}$, Kevin K. \\ Sheth ${ }^{2}$. \\ ${ }^{1}$ Yale School of Medicine, Department of Neurology, New Haven, CT, USA, ${ }^{2}$ Yale School of Medicine, \\ Department of Neurology, New Haven, USA.
}

\section{Introduction:}

Multiple intracerebral hemorrhage $(\mathrm{ICH})$ studies have examined differences between discharge and 3month outcome. However, few studies have examined late recovery specifically between 3- and 12month endpoints. The aim of this study was to identify potential factors predicting late recovery in $\mathrm{ICH}$ patients.

\section{Methods:}

Twenty-four patients diagnosed with primary $\mathrm{ICH}$ at Yale-New Haven Hospital were prospectively enrolled between July 1, 2014 and July 1, 2016. Outcomes were assessed using Barthel Index (BI) at discharge, 3 months, and 12 months. Repeated-measures regression analysis was conducted using age, admission Glasgow Coma Scale (GCS), ICH volume, intraventricular extension, and ICH location, to compare outcomes at discharge, 3 months, and 12 months.

\section{Results:}

There was a significant improvement across time points $(p=0.005)$, with follow-up testing showing improvement between discharge and 3 months $(p<0.001)$, discharge and 12 months $(p=0.036)$, and 3 months and 12 months $(p=0.021)$. Regression testing resulted in a significant relationship of time $(p=$ $0.002)$, time $\times$ GCS $(p=0.003)$, time $\times \mathrm{ICH}$ volume $(p<0.001)$, time $\times \mathrm{ICH}$ location $(p<0.001)$. Plots show increased late recovery (i.e., between 3 and 12 months) for patients with lower GCS scores, larger $\mathrm{ICH}$ volume, and deep ICH location.

\section{Conclusions:}

Patients with more devastating initial ICHs show greater gains in late recovery between 3 and 12 months. These results suggest initial disability at 3 months may not represent overall recovery and support continual follow-up out to one year. 


\title{
Poster 81
}

\section{Elderly Patients with Intracerebral Hemorrhage Have Higher Morbidity than but Similar Mortality to Younger Patients at Three Months.}

\author{
Audrey C. Leasure, Anirudh A. Sreekrishnan, Sonya S. Zhou, David D. Hwang, David D. Greer, Kevin K. \\ Sheth.
}

Yale School of Medicine, Department of Neurology, New Haven, CT, USA.

\section{Introduction:}

While extensive studies have examined the outcomes of intracerebral hemorrhage (ICH) patients under the age of 80 , few studies have examined outcomes in an elderly cohort ( $>80$ years). The aim of this case-control study was to determine the independent effects of age on outcome after ICH.

\section{Methods:}

Nineteen ICH patients prospectively identified at Yale-New Haven Hospital above the age of 80 were matched against 19 patients below age 80, based on ICH location (lobar, deep, cerebellar, brainstem), $\mathrm{ICH}$ volume (>30cC), presence of intraventricular hemorrhage (IVH), and admission GCS $(0-4,5-12,13-$ 15). The matched groups were compared via univariate analysis to examine differences in morbidity (defined as modified Rankin Scale > 3), mortality, and Barthel Index at discharge and 3 months.

\section{Results:}

While there was no difference in pre-ICH disability ( $\mathrm{mRS}>1 ; \mathrm{p}>0.05)$, at 3 months elderly patients exhibited higher morbidity ( $m R S>3, p=0.023$ ). Despite these differences, there were no differences in overall mortality between groups at discharge or at 3 months and no differences between Barthel Index at 3 months.

\section{Conclusions:}

Despite greater morbidity as defined by $\mathrm{mRS}$, elderly patients with $\mathrm{ICH}$ do not significantly differ from younger patients in mortality rates or ability to perform activities of daily living at discharge and 3 months. These results may warrant further studies to provide more accurate prognostication after ICH in elderly populations. 


\title{
Poster 82
}

\section{High Variability in Acute Blood Pressure Control In Intracerebral Hemorrhage}

\author{
Jody Manners ${ }^{1}$, Jonathan J. Elmer ${ }^{2}$, Yazan Y. Bittar ${ }^{3}$, Lori L. Massaro ${ }^{4}$, Lori L. Shutter ${ }^{5}$, Sherry S. Chou ${ }^{5}$. \\ ${ }^{1}$ University of Pittsburgh School of Medicine/Department of Neurology, Pittsburgh, PA, USA, ${ }^{2}$ University of \\ Pittsburgh School of Medicine/Emergency Medicine, Pittsburgh, PA, USA, ${ }^{3}$ University of Pittsburgh, \\ Pittsburgh, PA, USA, ${ }^{4}$ University of Pittsburgh Medical Center/Stroke Institute, Pittsburgh, PA, USA, ${ }^{5}$ \\ University of Pittsburgh School of Medicine/Critical Care Medicine, Pittsburgh, PA, USA.
}

\section{Introduction:}

Hypertension after intracerebral hemorrhage $(\mathrm{ICH})$ is associated with hematoma expansion, morbidity and mortality. There are currently no recommendations to standardize the critical care approach to acute blood pressure (BP) management in $\mathrm{ICH}$. We performed a large retrospective cohort study to examine practice variability in BP management in acute $\mathrm{ICH}$.

\section{Methods:}

We identified consecutive patients with spontaneous ICH admitted 12/2013 to 09/2015 to a single tertiary care center using local Get With The Guidelines data. We abstracted time-stamped clinical variables including all documented BP measurements, and medications administered, as well as hematoma location from the electronic medical record. All acute $\mathrm{ICH}$ patients are admitted to the neurolCU and comanaged by neuro-intensivists and vascular neurologists. We used descriptive statistics to summarize overall population and treatment characteristics.

\section{Results:}

Overall, 515 patients met inclusion criteria. Mean age was 70 (SD +/- 14) years, and $47 \%$ were female. Hemorrhage locations were $44 \%$ lobar, $34 \%$ deep, $14 \%$ brainstem/posterior fossa, $8 \%$ intraventricular and $1 \%$ other. Electronic medical record data were available in 497 subjects, of which $303(61 \%)$ received continuous infusion for BP control within 72 hours of admission ( $56 \%$ nicardipine, $9 \%$ clevidipine). A systolic BP goal was charted in $283(57 \%)$ and ranged from 120 to $220 \mathrm{mmHg}$. These goals were modified in $72 \%$ during the same admission (64\% increased, $8 \%$ decreased). Overall, 48 unique oral antihypertensives were administered (31\% received a beta-blocker, $22 \%$ a calcium-channel blocker, $18 \%$ an ACE inhibitor, and $11 \%$ a diuretic).

\section{Conclusions:}

Hypertension requiring continuous antihypertensive infusion is common after $\mathrm{ICH}$. There is high practice variability in BP targets and choice of antihypertensive medications. A prospective study of a systematic and protocolized approach to antihypertensive medication use in $\mathrm{ICH}$ is necessary to determine if reducing practice variability improves outcomes. 


\title{
Poster 83
}

\section{Intracerebral Hemorrhage and End-Stage Liver Disease: A Study of Long Term Outcomes}

\author{
Christopher P. Robinson', Joseph J. Zachariah², Eelco E. Wijdicks ${ }^{2}$.
}

${ }^{1}$ Mayo Clinic - Rochester Department of Neurology Division of Neurocritical Care, Rochester, MN, USA,
${ }^{2}$ Mayo Clinic - Rochester Department of Neurology Division of Neurocritical Care, Rochester, MN, USA.

\section{Introduction:}

Intracranial hemorrhage $(\mathrm{ICH})$ has long been thought to be a devastating consequence in the setting of end-stage liver disease. Due to its association with abnormal coagulation, the prognosis is thought to be poor, and frequently leads to withdrawal of care. Our aim with this study was to assess the true overall mortality rate of $\mathrm{ICH}$ in end-stage liver disease and compare it to mortality of $\mathrm{ICH}$ in the general population.

\section{Methods:}

All patients > 18 years of age admitted to Mayo Clinic from 2005 to 2015 with a subsequent diagnosis of non-traumatic $\mathrm{ICH}$ and end-stage liver disease were identified. Patients presenting with primary epidural, subdural, intraventricular, or subarachnoid hemorrhage were excluded. Using actuarial methods, 7 day, 30 day, and 1 year mortality rates were calculated.

\section{Results:}

112 patients with simultaneous diagnosis of $\mathrm{ICH}$ and end-stage liver disease were identified. 46 of the patients were female (41\%) and 66 patients were male (58\%). The mean age at diagnosis was 62 years. The mean systolic blood pressure in the mortality cohort was $162 \mathrm{mmHg}$, compared to $160 \mathrm{mmHg}$ in the survival cohort. The 7 day, 30 day, and 1 year mortality rates were $23 \%, 32 \%$, and $49 \%$ respectively.

\section{Conclusions:}

$\mathrm{ICH}$ associated mortality in end-stage liver disease does not significantly differ when compared to $\mathrm{ICH}$ mortality in the general population. The mean systolic blood pressure at presentation did not specifically correlate with an increased incidence of $\mathrm{ICH}$ associated mortality. These findings are important in the practice of neurocritical care, confirming that poor prognostication and expedited withdrawal of care should be reconsidered. Future directions will include mortality adjusted by hemorrhage location, size, and presence of intraventricular extension. 


\section{Poster 84}

\section{Assessment of Kcentra ${ }^{\circledR}$ Dosing Practices within Neurocritical Care}

Denise H. Rhoney ${ }^{1}$, Molly M. Merz ${ }^{1}$, Kathryn K. Morbitzer ${ }^{2}$, Aaron A. Cook ${ }^{3}$.

${ }^{1}$ UNC Eshelman School of Pharmacy, Chapel Hill, NC, USA, ${ }^{2}$ UNC Hospitals, Department of Pharmacy , Chapel Hill, NC, USA, ${ }^{3}$ University of Kentucky Medical Center, Department of Pharmacy, Lexington, KY, USA.

\section{Introduction:}

Kcentra ${ }^{\circledR}$ (human prothrombin complex concentrate) is the first FDA-approved non-activated 4-factor prothrombin complex concentrate for the urgent reversal of vitamin $\mathrm{K}$ antagonist agents in adults with acute major bleeding. The recommended dosing is based upon the units of Factor IX, which can vary within each vial (range from 20-31 units $/ \mathrm{mL}$ ). There is considerable variation in dosing and dispensing where some institutions may dispense based on the 500 units/vial where doses are rounded to nearest vial size to reduce waste and some based upon the exact Factor IX content. This variation in dosing may be associated with complications in care and has never been evaluated. Underdosing of the medication can lead to suboptimal response and overdosing the medication can cause thromboembolic events. The purpose of this observational trial is to assess the current prescribing practices of Kcentra ${ }^{\circledR}$ in neurocritical care unit patients across the United States and evaluate the impact on patient response and safety. Additionally we plan to characterize current approaches to repeat dosing.

\section{Methods:}

This was a retrospective observational study of adult patients across 39 centers who received Kcentra for reversal of warfarin-related bleeding between January 1, 2014 and December 31, 2015. Descriptive statistics and tests for comparison will be utilized to evaluate differences in dosing, outcome, and the occurrence of adverse events. Statistical significance will be defined as $p$-value $<0.05$.

\section{Results:}

Data collection is ongoing but the full results will be presented during the meeting.

\section{Conclusions:}

Result of this study will document the real-world use of Kcentra ${ }^{\circledR}$ in patients with severe life threatening bleeding and assess the impact of variations in prescribing practices on patient responses. There is a paucity of data regarding this topic and combining data from multiple neurocritical care units will be timely in identify optimal dosing strategies. 


\title{
Poster 85
}

\section{Perihematomal Edema Volume Predicts Poor Outcome at Discharge in Patients with Cerebellar Intracerebral Hemorrhage.}

\author{
Audrey C. Leasure, Anirudh A. Sreekrishnan, Sonya S. Zhou, Lauren L. Sansing, David D. Greer, Kevin \\ K. Sheth.
}

Yale School of Medicine, Department of Neurology, New Haven, CT, USA.

\section{Introduction:}

Perihematomal edema (PHE) associated with intracerebral hemorrhage (ICH) has been suggested to have an impact on both mortality and functional outcomes in spontaneous, supratentorial ICH. There have been no studies examining the impact of PHE in infratentorial hemorrhage. The aim of this study was to evaluate the impact of absolute PHE volume as well as PHE expansion rate in cerebellar hemorrhages at the time of discharge and at 3 months.

\section{Methods:}

9 patients diagnosed with primary cerebellar $\mathrm{ICH}$ at Yale New Haven Hospital were prospectively enrolled between July 1, 2014 and July 1, 2016. Patients were evaluated using $\mathrm{mRS}$ and $\mathrm{BI}$ at discharge and 3 months. ICH and edema volumes on CT were measured using a semi-automated threshold based approach and PHE expansion rate was the difference between initial and follow-up PHE volumes divided by the time interval. Univariate analysis was conducted to look at differences in admission Glasgow Coma Scale (GCS), ICH volume, intraventricular (IVH) volume, edema (PHE), and PHE expansion rate from baseline to first follow up CT scan before decompression (6h window).

\section{Results:}

At discharge, patients with higher morbidity (mRS $>3$ ) exhibited higher ICH volume $(p=0.012)$ and PHE volume $(p=0.005)$ on admission CT and a trend for greater rates of PHE expansion $(p=0.086)$. These differences were not significant at 3 months and when adjusted for $\mathrm{ICH}$ volume. Plots suggest an association between higher PHE rate and lower $\mathrm{BI}$ scores at 3 months.

\section{Conclusions:}

PHE may have a clinically significant impact in patients with cerebellar $\mathrm{ICH}$ at time of discharge, but these results are limited by a small sample size. These results warrant further studies and suggest therapies to ameliorate edema may be a treatment option for cerebellar $\mathrm{ICH}$. 


\title{
Poster 86
}

\section{Primary Acute Stroke Thrombectomy within 3 Hours for Large Artery Occlusion (PAST3-LAO) - A Pilot Study}

\author{
Yahia M. Lodi ${ }^{1}$, Varun V. Reddy ${ }^{2}$, Anas A. Hourani ${ }^{3}$, Nishant N. Ranawat ${ }^{4}$, Chun-An C. Chou ${ }^{5}$. \\ ${ }^{1}$ Upstate Medical University/UHS-Wilson Medical Center, Johnson City, NY, USA, ${ }^{2}$ UHS-Wilson Medical \\ Center/Upstate Medical University, Binghamton, Johnson City, NY, USA, ${ }^{3}$ Department of Systems \\ Science \& Industrial Engineering, Binghamton University, Binghamton, NY, USA, ${ }^{4} U H S$-Wilson Medical \\ Center/Upstate Medical University, Johnson City, NY, USA, ${ }^{5}$ Department of Systems Science \& Industrial \\ Engineering, Binghamton University, Bonghamton, NY, USA.
}

\section{Introduction:}

In recent trials, acute ischemic stroke (AIS) from large artery occlusion (LAO) is resistant to IV thrombolysis and adjunctive stent retriever thrombectomy (SRT) is associated with better perfusion and outcomes. Despite benefit, $39 \%$ to $68 \%$ of patients had poor outcomes. Thrombectomy in AIS with LAO within 3 hours is performed as secondary after IV thrombolysis, which may be associated with delay. The purpose of our study is to evaluate the safety, feasibility, recanalization rate and outcome of primary STR within 3 hours without intravenous thrombolytic in AIS from LAO.

\section{Methods:}

Based on institutionally approved protocol stroke patients with LAO within 3 hours were offered primary SRT as an alternative to IV rtPA. Consecutive patients who underwent primary SRT for LAO within 3 hours from 2012 to 2014 were enrolled. Outcomes were measured using modified Rankin Scale (mRS).

\section{Results:}

18 patients with LAO; mean age $62.83 \pm 15.32$ years and mean NIHSS $16 \pm 4$; chose primary SRT after informed consent. Near complete ( $\mathrm{TICl} 2 \mathrm{~b}$ in 1) complete ( $\mathrm{TICl} 3$ in 17) was observed in all $(100 \%)$ patients. Recanalization from symptoms and groin puncture was $188.5 \pm 82.7$ and $64.61 \pm 40.14$ minutes respectively. Immediate post-thrombectomy, 24 hour and 30 day NIHSS score was $4.44 \pm 3.75,1.9 \pm 3.2$ and $0.28 \pm 0.9$ respectively. Asymptomatic perfusion related hemorrhage developed in 4 patients (22\%). 90 days outcomes; mRS0 50\%, mRS1 44.4\%, and mRS2 5.6\%.

\section{Conclusions:}

Our study demonstrates that primary SRT in AIS from LAO occlusion is not only safe and feasible, but associated with complete recanalization and good outcome. Further study is required. 


\section{Poster 87}

\section{Early Prediction of Cerebral Herniation in Patients Presenting with Large Hemispheric Infarction}

Christopher Melinosky ${ }^{1}$, Shiming S. Yang ${ }^{2}$, Peter P. Hü ${ }^{2}$, Yao Y. Li $i^{2}$ Imad I. Khan ${ }^{1}$, Wan-Tsu W. Chang ${ }^{1}$, Gunjan G. Parikh ${ }^{1}$, Melissa M. Motta ${ }^{1}$, J. Marc J. Simard ${ }^{3}$, Neeraj N. Badjatia'.

\footnotetext{
${ }^{1}$ Neurocritical Care, Program in Trauma, Univ. of Maryland School of Medicine, Baltimore, MD, USA, ${ }^{2}$ Department of Anesthesiology, University of Maryland School of Medicine, Baltimore, MD, USA, Department of Neurosurgery, University of Maryland School of Medicine, Baltimore, MD, USA.
}

\section{Introduction:}

Currently, no reliable predictive tools are available to determine which patients with a large hemispheric infarction $(\mathrm{LHI})$ will progress to cerebral herniation $(\mathrm{CH})$. We sought to determine whether continuous measurements of blood pressure variance (BPV), heart rate variance (HRV), and entropy within 3 hours of admission would enhance the ability to predict future $\mathrm{CH}$ in $\mathrm{LHI}$ patients.

\section{Methods:}

Patients presenting within 24 hours of onset from an internal carotid artery (ICA) or middle cerebral artery (MCA) syndrome with NIHSS $\geq 15$ were selected from a registry of stroke admissions between January 2013 and November 2015. Patients with $\mathrm{CH}$ were matched 1:1 by age and NIHSS with patients who did not have $\mathrm{CH}$. Shannon entropy and standard deviation were used to measure the instability of HR and BP. The Wilcoxon rank sum test was used to assess the median difference of BPV, HRV, and entropy between groups. A model predicting $\mathrm{CH}$ utilizing the admission factors of age, gender, NIHSS, intraarterial (IA) therapy, and thrombolysis was compared to an admission model enhanced with BPV, HRV and entropy 3 hours after admission.

\section{Results:}

Data from 40 patients were analyzed (median age 60 years old, median NIHSS 19, 43\% women). There were no differences in the proportion of patients with a left hemisphere syndrome (45\%), undergoing IA therapy $(25 \%)$ or thrombolysis $(43 \%)$ between groups. $\mathrm{CH}$ was observed a median of 3 days after the ictus. At 3 hours after admission, median measures of BPV, HRV and entropy were significantly higher in the $\mathrm{CH}$ group $(\mathrm{P}<0.001)$. A model of admission factors enhanced with physiologic data was better able to predict $\mathrm{CH}$ than a model with admission factors alone (ROC:0.77 v. $0.63, \mathrm{P}=0.04$ )

\section{Conclusions:}

Dysautonomia measures are early markers of $\mathrm{CH}$ in $\mathrm{LHI}$ patients, and can enhance prediction of $\mathrm{CH}$ when combined with known clinical factors. 


\section{Poster 88}

\section{CSF Volumetrics to Model Early Kinetics of Malignant Cerebral Edema after Large Hemispheric Infarction}

Rajat Dhar, Kristy K. Yuan, Tobias T. Kulik, Yasheng Y. Chen, Jin-Moo J. Lee.

Neurology, Washington University in St. Louis, Saint Louis, MO, USA.

\section{Introduction:}

Early recognition of which patients with large hemispheric infarction (LHI) will develop malignant cerebral edema (MCE) would facilitate timely selection for interventions like hemicraniectomy prior to herniation. However, the early time-course of edema has not been adequately studied. We applied volumetric measurements of CSF volumes on serial CT scans to model edema kinetics. Our hypothesis was that trajectory of reduction in CSF volumes ( $\triangle \mathrm{CSF}$ ) would differ for those destined for MCE within the first 24 hours, prior to development of midline shift (MLS).

\section{Methods:}

We identified $38 \mathrm{LHI}$ patients with scans within six hours of onset and subsequent scans early (6-48 hours) and/or at peak edema (48-120 hours). Volumes of CSF compartments (sulci, ventricles, ipsilateral vs. contralateral to infarct) were measured and $\triangle \mathrm{CSF}$ calculated at each time point. Volume of infarctrelated hypodensity and MLS were also measured. $\triangle \mathrm{CSF}$ and MLS were modeled over time for those with vs. without MCE.

\section{Results:}

Median $\triangle$ CSF was $-50 \mathrm{ml}$ on peak edema scan (median 64 hours). Over half this reduction in CSF (-29 $\mathrm{ml}$ ) was seen by time of early scan (median 18 hours). In contrast, MLS was $4.5 \mathrm{~mm}$ at peak but only 0.5 $\mathrm{mm}$ on early scan. $\triangle \mathrm{CSF}$ within ipsilateral sulci and ratio of ipsi-to-contralateral sulcal volumes best distinguished those with MCE prior to 24 hours. In fact, our model demonstrated that the trajectory of MCE diverged from that observed in patients without MCE within 12 hours of stroke onset.

\section{Conclusions:}

This study applied $\Delta$ CSF to model the early kinetics of cerebral edema after LHI. We demonstrated that the majority of edema develops within the first day, prior to significant MLS and that this novel biomarker may differentiate those destined for MCE as early as 12 hours after onset. Prospective studies should evaluate its role in early selection of patients for aggressive interventions. 


\title{
Poster 89
}

\section{Intravenous rt-PA Use Prior to Endovascular Thrombectomy Increases Probability of Good Outcome in Acute Ischemic Stroke}

\author{
Pitchaiah Mandava ${ }^{1}$, Sharyl S. Martini ${ }^{1}$, Vishank V. Shah ${ }^{2}$, Roderic R. Fabian ${ }^{1}$, Thomas T. Kent ${ }^{1}$. \\ ${ }^{1}$ Baylor College of Medicine and Michael E. DeBakey VA Medical Center, Houston, TX, USA, ${ }^{2}$ Baylor \\ College of Medicine, Houston, TX, USA.
}

\section{Introduction:}

While all 6 recently published endovascular stent-retriever randomized clinical trials (RCTs) were positive, their designs differed considerably particularly with regard to the extent of intravenous rt-PA use prior to thrombectomy. Here, we assessed whether rt-PA use influenced likelihood of good outcome.

\section{Methods:}

We adapted a method previously published by us to develop a pooled outcome model relating percent utilization of rt-PA to outcome. Our method is based on the control arms of 55 stroke RCTs representing > 11,000 subjects and a range of $0-100 \%$ utilization of rt-PA. We correlated percent rt-PA and baseline stroke severity (NIHSS) with chance for good functional outcome (modified Rankin Score, mRS 0-2) and mortality. This model includes $\pm p<.05$ statistical interval surfaces to assess whether a trial's outcomes surpasses the variability of the pooled sample (Neurology 85:274-83, 2015). Stent retriever RCTs were compared against the model.

\section{Results:}

The mRS model showed excellent fit: R-square $=0.83, p<0.001$. Each stent retriever trial's outcomes exceeded mRS 0-2 +95\% surface confirming better outcomes than without thrombectomy. The degree of improvement varied dramatically according to \%rt-PA, with the greatest improvement in those with $100 \%$ rt-PA use. When we included all case series and testing their outcomes at their baseline NIHSS and percent rt-PA use, shortest overall treatment times also related to improved outcomes $(p=.011)$. Mortality trends were similar in that lowest mortality was seen in those trials with highest rt-PA use and the trial with higher than expected mortality had the lowest rt-PA use.

\section{Conclusions:}

An outcome model including \% IV rt-PA permitted analysis of stent-retriever therapy compared to a large sample. Best outcomes were related to higher rt-PA utilization and shorter treatment times, suggesting a strong treatment interaction between modalities. Based on these results, until studied prospectively, IV rtPA, if administered rapidly, should not be bypassed prior to thrombectomy. 


\title{
Poster 90
}

\section{Safety and Efficacy of Mechanical Thrombectomy Under General Anesthesia for Acute Ischemic Strokes}

\author{
ABDULLAH S. ALAMRI, HISHAM H. ALTHUKAIR, ABDULRAHMAN A. ALTURKI, ALI A. ALAMER, \\ DONATELLA D. TAMPIERI, JUSTIN J. LETOURNEAU, MOHAMMAD M. BADAWI, BENJAMIN B. LO, \\ MARK M. ANGLE, JEANNE J. TEITELBAUM.
}

MONTREAL NEUROLOGICAL HOSPITAL, MONTREAL, Canada.

\section{Introduction:}

Intra-arterial mechanical thrombectomy (IAMT) is currently considered the standard therapy for acute ischemic strokes (AIS) involving the anterior cerebral circulation. Recent studies suggest that thrombectomies performed under general anesthesia (GA) may adversely affect functional outcomes. We report our experiences with IAMT performed routinely under GA at the Montreal Neurological Hospital $(\mathrm{MNH})$.

\section{Methods:}

This is a retrospective analysis of adult patients admitted to the MNH from January 2015 to April 2016 with AIS from proximal intracranial occlusions of the anterior cerebral circulation. All patients were assessed both clinically and radiologically.

\section{Results:}

Forty-two patients underwent IAMT for AIS. Twenty-eight patients were included for analyses (4 had procedures under conscious sedation, 10 had missing 3 months assessment). Clinical outcomes were classified based on the modified Rankin Scale score (mRS) 3-month post stroke. 16/28 (57\%) had good outcomes (mRS 0-2) and 12 had poor outcomes. Average time from stroke onset to first recanalization was 227 minutes (including 3 wake-up strokes) and 266 minutes (including 5 wake-up strokes) for the good and poor outcome groups respectively. Sixty-eight percent of the good outcome group received IV tPA, as compared to $50 \%$ in the poor outcome group. Patients in the good outcome group were also more likely to be managed with laryngeal mask airways [ $75 \%$ versus $58 \%$ in the poor outcome group]. Intraprocedural MAP drops below 60 mmh observed in 3 patients in the good outcome group versus non in the poor outcome group.

\section{Conclusions:}

In our small retrospective single centre study about thrombectomy for AIS under GA, our 3-month outcomes are comparable to larger studies where thrombectomies were done under conscious sedation. Pending the results of ongoing prospective trials about the use of GA during IAMT for AIS, our results do not support the reservations derived from recently published retrospective data on the use of GA in this context. 


\title{
Poster 91
}

\section{Conservative initial management of young adults with severe hemispheric stroke in a comprehensive stroke center reduces decompressive craniectomy rates}

\author{
Paul T. Akins ${ }^{1}$, Yekaterina Y. Axelrod ${ }^{2}$, Syed S. Arshad ${ }^{2}$, Kern K. Guppy². \\ ${ }^{1}$ Department of Neurosurgery Permanente Medical Group Kaiser Sacramento Medical Center, \\ Sacramento, CA, USA, ${ }^{2}$ Department of Neurosurgery Permanente Medical Group Kaiser Sacramento, \\ Sacramento, CA, USA.
}

\section{Introduction:}

Pooled European trial results of early decompressive craniectomy (DC) did not require radiographic mass effect at the time of DC. Early surgery for supratentorial cerebral hemorrhage does not improve recovery or survival compared to initial conservative medical management. Early vs delayed DC for hemispheric stroke has not been investigated as rigorously.

\section{Methods:}

A prospective inpatient neurosurgical database from October 2007 to March 2015 was queried for neurocritical care admissions for hemispheric ischemic stroke in patients aged 18-60 under IRB approval. A retrospective chart review was conducted using a structured questionnaire using the electronic medical record.

\section{Results:}

We identified 30 patients who met the inclusion criteria for the pooled European DC stroke trial. The mean age was 46 , and the median $\mathrm{NIH}$ stroke score was 19 . All hemispheric stroke patients were monitored with hourly neurochecks and daily CT scans for a minimum of 3 days. Eighteen (60\%) patients were managed with medical treatment only (MTO) with average maximal septal shift of $5.2 \mathrm{~mm}$ and pineal shift of $3.1 \mathrm{~mm}$. Twelve patients (40\%) underwent DC with average maximal septal shift of $6.8 \mathrm{~mm}$ and pineal shift of $4.1 \mathrm{~mm}$. Modified Rankin (MR) outcomes at 3 months for the overall group, MTO, and DC were respectively: MR $0-360 \%$ vs $67 \%$ vs $50 \%$; MR $4-527 \%$ vs $17 \%$ vs $42 \%$, and death $13 \%$ vs $17 \%$ vs $8 \%$. Four patients in the MTO group declined DC; 3 died and one survived with MR of 4 . No patients developed brainstem herniation prior to referral for decompressive craniectomy. Surgical complications occurred in $4 / 12(33 \%)$ patients.

\section{Conclusions:}

Delayed DC for hemispheric stroke is a safe alternative to early, prophylactic DC for young adults admitted to a comprehensive stroke center. This strategy reduced DC rates by $60 \%$ without any excess of death or survival with severe disabilities. 


\title{
Poster 92
}

\section{Time of recanalization since symptoms is a strong predictor of outcome in patients who underwent stent retriever thrombectomy from middle cerebral artery occlusion.}

\author{
Yahia M. Lodi ${ }^{1}$, Varun V. Reddy ${ }^{2}$, Anas A. Hourani ${ }^{3}$, Nishant N. Ranawat ${ }^{4}$, Chun-An C. Chou ${ }^{5}$. \\ ${ }^{1}$ Upstate Medical University/UHS-Wilson Medical Center, Johnson City, NY, USA, ${ }^{2}$ UHS-Wilson Medical \\ Center/Upstate Medical University-Binghamton, Johnson City, NY, USA, ${ }^{3}$ Department of Systems \\ Science \& Industrial Engineering, Binghamton University, Binghamton, NY, USA, ${ }^{4}$ UHS-Wilson Medical \\ Center/Upstate Medical University, Binghamton, Johnson City, NY, USA, ${ }^{5}$ Department of Systems \\ Science \& Industrial Engineering, Binghamton, NY, USA.
}

\section{Introduction:}

The outcome of acute ischemic stroke (AIS) patients who recanalize less than 3 hours versus more than 3 hours since symptoms have not been clearly investigated especially, those with large artery occlusion (LAO) and underwent stent retriever thrombectomy (SRT) . Objective: To identify the predictors of outcome in AIS patients from middle cerebral artery (MCA) occlusion with large clot burden (LCB >8 mm) and underwent SRT, who recanalize less than 3 hours versus more than 3 hours since symptoms.

\section{Methods:}

Consecutive AIS patients with MCA occlusion with NIHSS $\geq 10$ who underwent SRT were enrolled from 2012 to 2014. Outcome was measured using modified Rankin Scale (mRS) at 30 days. The SAS software was used to analyze the data.

\section{Results:}

21 AIS patients who underwent SRT in MCA; age $70.62 \pm 13.94$ years and mean admission NIHSS $17 \pm 5$. Complete $(\mathrm{TICl} 3)$ and partial $(\mathrm{TICl} 2 \mathrm{~b})$ recanalization was observed in $90.50 \%$ and $9.50 \%$ respectively with mean passes of $1.71 \pm 1$. Time to recanalization from stroke onset was $230 \pm 160$ minutes. Presenting NIHSS of 16.76 dropped to 7, 5 and 2 at immediate, 24 hours and 30 days post SRT respectively. Good outcome (mRS $\leq 2$ ) was observed in $66.13 \%$ and poor outcome in $28.57 \%$ including mortality in $9.5 \%$. In univariate analysis, recanalization time, immediate and 24 hours post SRT NIHSS were predictors of outcome ( $p$-value $=0.0039,0.003$ and 0.043 respectively). In multivariate analysis, time of recanalization since symptoms ( $p$-value $=0.01)$ and baseline mRS ( $p$-value $=0.01)$ continued to be the predictors of good outcome.

\section{Conclusions:}

Our study demonstrates that patients with LAO from MCA who recanalize less than 3 hours of symptoms onset have good chance of good outcome compared to those who recanilize more than 3 hours. Therefore, all AIS patients with LAO should offer early SRT to achieve a good functional outcome. Further studies are required. 


\title{
Poster 93
}

\section{Infection and Stroke Risk following Continuous Flow Left Ventricular Assist Device Implant}

\author{
Jennifer A. Frontera. \\ Neurological Institute, Cleveland Clinic, Cleveland, OH, USA.
}

\section{Introduction:}

Infection has been associated with stroke in patients with left ventricular assist devices (LVAD), however, the timing and type of infection associated with specific stroke subtypes has not been investigated.

\section{Methods:}

Prospectively collected data of Heartmate II $(\mathrm{N}=332)$ and Heartware $(\mathrm{N}=70)$ LVAD patients from a single center were reviewed from 10/21/2004-5/19/2015. The association of LVAD infections (wound infection, pump pocket infection, driveline infection and blood stream infection [BSI]), specific pathogens (Pseudomonas and Staphylococcus aureus) and ischemic and hemorrhagic stroke were evaluated using Mann-Whitney $\mathrm{U}$, Chi-squared tests and multivariable, backward, step-wise logistic regression analyses.

\section{Results:}

Of 402 patients, LVAD infection occurred in 158 (39\%) including: BSI in 107 (27\%), wound infection in 31 (8\%), pump pocket infection in $24(6 \%)$ and driveline infection in $67(17 \%)$. In the entire cohort, stroke occurred in 69/402 (17\%) patients. Stroke occurred in 36/158 $(23 \%)$ of those with LVAD infection and in $33 / 244(14 \%)$ of those without LVAD infection $(P=0.016)$. Among these, ischemic stroke occurred in $22 / 158(14 \%)$ and hemorrhagic stroke in $20 / 158(13 \%)$. Predictors of ischemic stroke included any infection during hospitalization for LVAD implant, multiple infections, pump pocket infections and BSI (all $\mathrm{P}<0.05)$. Predictors of hemorrhagic stroke included any infection after hospital discharge, and BSI (both $\mathrm{P}<0.05$ ). Driveline and wound infection were not associated with stroke. In multivariable analysis, ischemic stroke was associated with any infection during hospitalization (aOR 2.3, 95\% $\mathrm{Cl} 1.2-4.5$, $\mathrm{P}=0.012$ ) and pump pocket infection (aOR 3.0,95\% Cl 1.1-8.1, $\mathrm{P}=0.034)$, while hemorrhagic stroke was associated with $\mathrm{BSI}(\mathrm{aOR} 2.6,95 \% \mathrm{Cl} 1.3-5.1, \mathrm{P}=0.005)$. There was no association with any specific infectious pathogen.

\section{Conclusions:}

LVAD infections, particularly BSI and pump pocket infections are significantly associated with stroke. Precautions to mitigate infection risk may reduce stroke rates, but further research is necessary to demonstrate a causal relationship. 


\title{
Poster 94
}

\section{ENTERAL TUBE FEEDING IN ACUTE ISCHEMIC STROKE PATIENTS: A MULTIVARIATE PREDICTIVE ANALYSIS.}

\author{
Lais M. Medeiros ${ }^{1}$, Maira M. Honorato ${ }^{1}$, Luiz Fernando L. Oliveira ${ }^{1}$, Michel M. Machado ${ }^{1}$, Thomaz T. \\ Yasuda ${ }^{1}$, Luiz L. Dalfior Junior', Maria Sheila M. Rocha ${ }^{2}$. \\ ${ }^{1}$ Hospital Santa Marcelina. Department of Neurology. Neurovascular Unit, Sao Paulo, Brazil, ${ }^{2}$ Hospital \\ Santa Marcelina. Department of Neurology. Neurovascular Unit., Sao Paulo, Brazil.
}

\section{Introduction:}

Stroke is the most frequent cause of neurogenic oropharyngeal dysphagia. In the acute phase of stroke, the frequency of dysphagia is greater than $50 \%$. The early clinical evaluation of swallowing disorders can help define approaches and avoid oral feeding, which may be detrimental to the patient. The aim of this study was to identify predictive clinical factors associated with enteral tube feeding in acute ischemic stroke patients.

\section{Methods:}

The medical records of 326 acute ischemic stroke patients from our database were reviewed. Clinical factors as age, sex, comorbidities, blood pressure, glycemia, National Institutes of Health Stroke Scale (NIHSS) score and subscores, Glasgow Come Scale (GCS), previous Rankin, Alberta Stroke Program Early CT score (ASPECTS) and localization of acute stroke were analyzed. Logistic regression was used to develop a risk score by weighting predictors of enteral tube feeding placement based on strength of association.

\section{Results:}

Of the 326 patients, 84 used enteral feeding tubes (25.8\%). The mean age (70.2 years - SD 13.1), mean GCS (12.7 - SD 2.1), mean NIHSS (12.6 - SD 5.6), and Aspect score (8.8 - SD 1.8) were significantly higher in the tube group. Logistic regression showed that only age (odds ratio [OR], 1.03; 95\% confidence interval $[\mathrm{Cl}], 1.00-1.6 . \mathrm{P}=0,025)$, NIHSS score $(\mathrm{OR}, 1.15 ; 95 \% \mathrm{Cl}, 1.05-1.25, \mathrm{P}=0,001)$ and $\mathrm{NIHSS} 10$ (dysarthria) subscore $(\mathrm{OR}, 2.2 ; 95 \% \mathrm{Cl}, 1.2-4.05, \mathrm{P}=0,011)$ were independent predictors of enteral tube feeding. A 3-item risk score was developed based on the regression model in order to identify those patients needing enteral feeding. A score $\geq 4$ predicted tube feeding in $75 \%$ of cases.

\section{Conclusions:}

In conclusion, combining information about age, NIHSS, NIHSS 10 subscore, may be a useful predictor tool for clinical decision for enteral tube feeding in acute ischemic stroke patients. 


\title{
Poster 95
}

\section{“Task Calc. Stroke” Tool: Managing IV t-PA Tasks Effectively for Acute Stroke Patients}

\author{
Terutake Yonemori ${ }^{1}$, Shoji S. Matsumoto ${ }^{2}$, Hiroshi H. Koyama ${ }^{3}$, Jun-ichi J. Kira ${ }^{4}$.
}

\author{
${ }^{1}$ Critical Care Medical Center, Urasoe-city, Japan, ${ }^{2}$ Director of Neurology, Kokura Memorial Hospital, \\ Kitakyushu, Japan, ${ }^{3}$ Adcanced Institute of Industrial Technology, Tokyo Metropolitan University, Tokyo, \\ Japan, ${ }^{4}$ Kyushu university, Fukuoka, Japan.
}

\section{Introduction:}

Tissue plasminogen activator (t-PA) treatment for acute ischemic stroke patients requires several medical tasks that should be completed within 60 minutes after hospital arrival. However, efficiently processing these tasks is difficult in most hospitals. We designed and developed an Information and Communication Technology (ICT) system, "Task Calc. Stroke," to support the processing of these tasks.

\section{Methods:}

"Task Calc. Stroke" consists of ICT technology, such as cloud environments, smart devices, Apple iPads, PCs, and big-screen digital signage. By clicking one button, it broadcasts messages to hospital medical staff, including stroke physicians, medical technologists, and nurses, and it immediately activates many departments before the patient's arrival. Required tasks appear on the screen, and task progress is indicated by color changes. Medical staff can prepare their own tasks before the patient's arrival, and the number of calls is reduced.

\section{Results:}

We compared the number of times that emergency room (ER) nurses called for computed tomography (CT) or magnetic resonance imaging (MRI) between before and after the system introduction. Before this system, ER nurses called for CT or MRI an average 3.8 and 4.2 times, respectively; after system introduction, the average number of times decreased to 1.2 and 1.8, respectively. Therefore, this system can improve the efficiency of task management, and the stroke physician can monitor the progress of all tasks and decide when to use intravenous (IV) t-PA. Also, it automatically records the transitions between and times for all tasks during acute stroke care, and it aggregates the performance of care. Therefore, this tool can help improve the quality of acute stroke care.

\section{Conclusions:}

"Task Calc. Stroke" could improve treatment times for IV t-PA in many stroke centers worldwide owing to early preparation, cooperation among staff, and recognition of task progression. We are planning a clinical trial to confirm the tool's efficacy. 
Poster 96

\title{
SEDATION AND AIRWAY DURING ENDOVASCULAR TREATMENT OF ACUTE ISCHEMIC STROKE: PRIMARY ENDPOINT RESULTS FROM THE RANDOMIZED TRIAL SIESTA (SEDATION VS INTUBATION FOR ENDOVASCULAR STROKE TREATMENT)
}

\author{
Silvia Schönenberger ${ }^{1}$, Lorenz L. Uhlmann ${ }^{2}$, Simon S. Schieber ${ }^{1}$, Sibu S. Mundiyanapurath ${ }^{1}$, Jan J. \\ Purrucker $^{3}$, Simon S. Nagel ${ }^{1}$, Johannes J. Pfaff ${ }^{4}$, Christina C. Klose ${ }^{5}$, Meinhard M. Kieser ${ }^{5}$, Martin M. \\ Bendszus ${ }^{6}$, Peter P. Ringleb ${ }^{3}$, Werner W. Hacke ${ }^{3}$, Markus M. Möhlenbruch ${ }^{6}$, Julian J. Bösel ${ }^{3}$. \\ ${ }^{1}$ University Hospital Heidelberg/Department of Neurology, Heidelberg, Germany, ${ }^{2}$ University Hospital \\ Heidelberg/Institute of Medical Biometry and Informatics, Heidelberg, Germany, ${ }^{3}$ University Hospital \\ Heidelberg/ Department of Neurology, Heidelberg, Germany, ${ }^{4}$ University Hospital Heidelberg/Department \\ of Neuroradiology, Heidelberg, Germany, ${ }^{5}$ University Hospital Heidelberg/ Institute of Medical Biometry \\ and Informatics, Heidelberg, Germany, ${ }^{6}$ University Hospital Heidelberg/ Department of Neuroradiology, \\ Heidelberg, Germany.
}

\section{Introduction:}

An important and controversial issue of peri-interventional management of endovascular stroke treatment (EST) is the management of sedation and airway. According to retrospective data the widely favored intubation and general anesthesia (GA) appears associated with worse functional outcome compared to "conscious sedation" (GS) in the non-intubated state.

\section{Methods:}

SIESTA is a prospective, monocentric, outcome assessor-blinded, 1:1 randomized, parallel-group interventional study comparing non-intubated vs. intubated patients receiving EST for acute ischemic stroke of the anterior circulation (www.clinicaltrials.gov, NCT 02126085, protocol published ${ }^{* *}$ ). Primary endpoint is the improvement of the National Institute of Health Stroke Scale (NIHSS) after 24 hours.

\section{Results:}

Enrolment of the intended 150 patients has been completed. Sixty of the recruited patients are female and 90 male, with a median baseline National Institutes of Health Stroke Scale (NIHSS) score of 17. In these patients, $64 \%$ received pre-procedural rtPA. Seventy-eight patients were randomized to CS, 11 $(14 \%)$ of these had to be converted to GA during the procedure. We will present preliminary results of the study, including the primary endpoint improvement in NIHSS after 24 hours and selected secondary endpoints.

\section{Conclusions:}

The aim of this study is the prospective randomized investigation of potential advantages of the nonintubated compared to the intubated state in stroke patients receiving EST. 


\section{Poster 97}

\section{Monitoring of Midline shift in Large Hemispheric Infarction during Therapeutic Hypothermia ; As an Outcome predictor}

In Young Chung.

Departments of Neurology1, Seoul National University Bundang Hospital, Seongnam, Korea, Republic of.

\section{Introduction:}

Patients presenting with large hemispheric infarction are likely to develop severe cerebral edema and that puts them poor prognosis like cerebral herniation and death. The authors analyzed the progression of cerebral edema during therapeutic hypothermia using the image markers to evaluate effect of therapeutic hypothermia.

\section{Methods:}

The authors retrospectively reviewed 21 patients with large hemispheric infarction who were treated with therapeutic hypothermia and hyperosmolar therapy from 2011 to 2014. Patients who were dead or underwent hemicraniectomy was defined as failure of therapeutic hypothermia. Infarction size was measured as sum of restricted area in diffusion weighted imaging which were performed on admission. B -CT was carried out regularly after onset of therapeutic hypothermia. Shift of septum pellucidum, pineal gland and choroid plexus calcification were measured in B-CT.

\section{Results:}

Seventeen patients were enrolled after exclusion of 4 patients whose B-CT was inadequate to evaluate. Ten patients were successfully treated with therapeutic hypothermia (group success, $n=10$ ). Six patients were dead and 1 patient had hemicraniectomy (group failure, $n=7$ ). Initial infarction size between two groups was not significantly different. Both septum pellucidum shift (SDS) and pineal gland shift (PGS) were significantly different in groups on $2.5 \pm 0.5$ days after onset of therapeutic hypothermia (mean SDS 5.0 vs. $14.9 \mathrm{~mm}$; mean PGS 2.3 vs. $7.9 \mathrm{~mm}$ ). Specificity and positive predictive values for the failure caused by cerebral edema of SDS $\geq 10 \mathrm{~mm}$ and $P G S \geq 6 \mathrm{~mm}$ on $2.5 \pm 0.5$ days were 1.0 . Choroid plexus calcification shift was not significantly different in groups during therapeutic hypothermia.

\section{Conclusions:}

Degree of progression of cerebral edema on $2.5 \pm 0.5$ days after onset of therapeutic hypothermia helps to estimate success or failure of treatment. Especially, SDS $\geq 10 \mathrm{~mm}$ and PGS $\geq 6 \mathrm{~mm}$ on $2.5 \pm 0.5$ days were excellent in predicting fatal outcome. The main limitation of this study include its retrospective singlecenter nature, which may limit generalizablility of the study. 


\title{
Poster 98
}

\section{Aminocaproic Acid for Reversal of Tissue Plasminogen Activator (tPA) Related Hemorrhagic Transformation in Acute Ischemic Stroke.}

\author{
Yana Bukovskaya ${ }^{1}$, Giuseppe G. Ciccotto ${ }^{2}$, Bethany B. Jennings ${ }^{3}$, Stephanie S. Faciane ${ }^{3}$, Harold H. \\ McGrade ${ }^{4}$, Alan A. Velander ${ }^{5}$, Vivek V. Sabharwal ${ }^{6}$, Ifeanyi I. Iwuchukwu ${ }^{5}$.
}
${ }^{1}$ Ochsner Medical Center; Department of Pharmacy, New Orleans, LA, USA, ${ }^{2}$ Ochsner Medical Center; Department of Neurocritical Care, New Orleans, LA, USA, ${ }^{3}$ Ochsner Medical Center; Department of Neurology, New Orleans, LA, USA, ${ }^{4}$ Ochsner Medical Center; Department of Neurocritical Care, Department of Neurology,, New Orleans, LA, USA, ${ }^{5}$ Ochsner Medical Center; Department of Neurocritical Care, Department of Neurology, New Orleans, LA, USA, ${ }^{6}$ Ochsner Medical Center; Department of Neurocritical Care, Department of Anesthesia, New Orleans, LA, USA.

\section{Introduction:}

Thrombolytic therapy increases the risk of hemorrhagic transformation $(\mathrm{HT})$ after acute ischemic stroke. There is limited knowledge on therapeutic treatment of post-thrombolytic ICH. Aminocaproic acid (ACA) inhibits binding of plasminogen to fibrin, hence inhibiting fibrinolytic property of tPA. There is limited knowledge of its benefits and risks in clinical use despite being an acceptable option for tPA reversal. We report a case series of ACA use for reversal of post-tPA HT.

\section{Methods:}

We reviewed the stroke database of our comprehensive stroke center from January 2013 to March 2016 and identified patients treated with IV tPA. Patients with post-tPA HT who received IV ACA were identified. Data on demographics, clinical characteristics, NIHSS, ICH score, new thrombotic events during hospitalization, and hospital and intensive care unit (ICU) length of stay (LOS) were collected.

\section{Results:}

A total of 36 patients developed post-tPA ICH, 12 of which received ACA. $58 \%$ of patients were male, mean age of $70.5 \pm 9.8$ years. Patients received tPA within a mean time of $153 \pm 43$ minutes from symptom onset, pre-tPA mean NIHSS was $11.6 \pm 5.7$. Mean time for HT after tPA administration was $2.6 \pm 0.7$ hours, with a hematoma volume of $45.5 \pm 58.3 \mathrm{~mm} 3$. In addition to ACA, $92 \%$ received cryoprecipitate, $67 \%$ platelets, and $17 \%$ fresh frozen plasma transfusions. $50 \%$ of patients had no hematoma expansion and $33 \%$ developed a new thrombotic event. Mean hospital LOS was $18 \pm 15$ days and mean ICU LOS was $14 \pm 9$ days. At the time of discharge $25 \%$ had an mRS of $4,33 \%$ mRS 5 and $42 \%$ mRS 6 .

\section{Conclusions:}

In this retrospective case series $50 \%$ of patients had hematoma expansion despite receiving ACA, while $33 \%$ had a new thrombotic event. Further research is warranted to determine the utility of ACA for the treatment of post-tPA HT. 


\section{Poster 99}

\section{Does Prior Use of Anti-Platelet Agents Matter for Endovascular Therapy for Acute Ischemic Stroke and subsequent 3-Month Functional Outcome?}

Sanjeev R. Keshary, Matthew M. Sharrock, Rawan R. Albaradeen, Manoj M. Mittal, Michael M. Abraham.

The University of Kansas Medical Center, Kansas City, KS, USA.

\section{Introduction:}

The American Heart Association recommends people over age 55 and cardiovascular risk factors take a daily aspirin to reduce risk of acute ischemic stroke (AIS). Patients still suffer from AIS secondary to a large vessel occlusion (LVO). While studies have analyzed difference in blood clot constructs, limited data is available understanding the effect of prior anti-platelet use on endovascular therapy (EVT) for IAS patients with LVO in the middle cerebral artery (MCA). We aimed to determine if prior anti-platelet use had effect on EVT procedure time, recanalization rate, and functional outcome measured by the modified Rankin Scale (mRS) after EVT for patients suffering from AIS secondary to large vessel occlusion.

\section{Methods:}

A retrospective chart review was conducted of 63 consecutive AIS patients who underwent EVT of LVO of MCA at the University of Kansas Medical Center from 2008-2014. Outcomes were measured using total procedural and fluoroscopy time, procedural recanalization score using the modified thrombolysis in cerebral infarction score $(\mathrm{TICl})$, time to recanalization, incidence of $\mathrm{sICH}$, and 3-month $\mathrm{mRS}$. Univariable and multivariable analysis were performed.

\section{Results:}

$29(46 \%)$ patients used antiplatelets prior to admission. Patients with antiplatelet use versus those without differed in age (72.9 vs 64.2 years, $p=0.02)$, history of coronary artery disease $(41.4 \%$ vs $8.8 \%, p=0.002)$, history of hypertension $(89.7 \%$ vs $58.9 \%, p=0.004)$, history of hyperlipidemia $(82.8 \%$ vs $41.2 \%$, $p=0.0006)$, use of statins $(65.5 \%$ vs $17.7 \%, p 0.05$ for all) were similar between antiplatelet use versus those without. In separate multivariable models (adjusting for all significant variables), antiplatelet use was not associated with tandem ICA occlusion, total procedure time, fluoroscopy time, good recanalization, or 3-month $\mathrm{mRS}$.

\section{Conclusions:}

No impact was found of prior anti-platelet use on stroke characteristics, procedural time, and functional outcomes in AIS patients undergoing EVT. These findings should be further confirmed in a larger database and prospective cohort study. 
Poster 100

\title{
The Effect of a Neurology-Specific Heparin Protocol with Narrowed Goal PTT Range on Rate of Hemorrhagic and Thromboembolic Events in Acute Ischemic Stroke: a Pre- and Post-Protocol Implementation Study.
}

\author{
Virginia M. Roberts ${ }^{1}$, Prachi P. Bhatt ${ }^{2}$, Lindsay L. Arnold ${ }^{2}$, Burns B. Joseph ${ }^{3}$, Anna A. Cervantes- \\ Arslanian $^{4}$.
}

${ }^{1}$ Boston Medical Center, Department of Neurology, Boston, MA, USA, ${ }^{2}$ Boston University School of Medicine and Boston Medical Center, Department of Pharmacy, Boston, MA, USA, ${ }^{3}$ Lahey Hospital and Medical Center, Department of Neurology, Burlington, MA, USA, ${ }^{4}$ Boston U. School of Medicine \& Boston Medical Center, Depts of Neurology \& Neurosurgery, Boston, MA, USA.

\section{Introduction:}

Data regarding optimal heparin infusion target PTT ranges in ischemic stroke are sparse, but suggest that PTT values above twice normal are associated with increased risk of hemorrhagic complications. This study evaluated the effect of a neurology-specific heparin infusion protocol with more frequent PTT monitoring and a narrower goal PTT range $(1.5 \mathrm{x}$ normal) on rate of hemorrhagic or thromboembolic events.

\section{Methods:}

This is a retrospective cohort study evaluating patients before (October 2011-September 2013) and after (October 2013-September 2014) implementation of a neurology-specific heparin infusion protocol. All patients $>18$ years old receiving intravenous heparin with a diagnosis of acute ischemic stroke were evaluated for inclusion. Primary outcomes are time to first therapeutic PTT and time to therapeutic PTT range. Secondary outcomes include rate of intracranial hemorrhage, rate of thromboembolic events, protocol compliance, number of subtherapeutic and supratherapeutic PTT values, time to initiation of oral anticoagulation, duration of heparin infusion, and number of heparin titrations.

\section{Results:}

Time to therapeutic PTT range was 18.7 hours in the pre-protocol group $(n=108)$ and 17.9 hours in the post-protocol group $(n=22)(p=0.049)$. Number of PTT values per patient was 20.2 in the pre-protocol group and 33.8 in the post-protocol group, of which $27.5 \%$ and $44.4 \%$ were therapeutic, respectively. Percentage of supratherapeutic PTT values was $22.2 \%$ and $16 \%$, respectively $(p=0.025)$. Time to first PTT, time to first therapeutic PTT, and percentage of subtherapeutic PTT values were not significantly different. Assessment of secondary clinical outcomes is ongoing.

\section{Conclusions:}

Our neurology-specific heparin protocol resulted in a faster time to therapeutic PTT range with a higher percentage of therapeutic PTT values and fewer supratherapeutic PTT values. Investigation regarding change in incidence of hemorrhagic and thromboembolic complications is ongoing. 
Poster 101

\title{
THROMBOLYSIS INTRAVENOUS WITH ALTEPLASE IN ISCHEMIC STROKE, BY SUPPORT NEUROLOGIST TELEMEDICINA EXPERIENCE IN MOYSES DEUTSCH HOSPITAL
}

\author{
ANA HELENA V. ANDRADE ${ }^{1}$, PATRICIA P. BULGARELLI' ${ }^{2}$ VALESCA V. GONZALEZ ${ }^{2}$, VINICIUS V. \\ AGIBERT', JULIO J. ARAUJO'. \\ ${ }^{1}$ HOSPITAL MOYSÉS DEUTSCH, SAO PAULO, Brazil, ${ }^{2}$ HOSPITAL MUNICIPAL MOYSÉS DEUTSCH, \\ SÃO PAULO, Brazil.
}

\section{Introduction:}

Hospital Moyses Deutsch, in the southern city of São Paulo is indicated for the use of rtPA intravenously in patients with ischemic stroke since 2011. Objective to demonstrate experience the use of intravenous alteplase with Neurologist Telemedecine support, checking the efficiencies and procedure safety.

\section{Methods:}

Retrospective study, in all cases of ischemic stroke who received intravenous alteplase, from December 2011 to February 2016. Protocol indicates the use of alteplase patients with inclusion criteria, the period between the onset of symptoms and hospital admission up to 4.5 hours and no contraindication to the use of thrombolytics, NIHSS calculated on admission and 24 hours after thrombolysis. Computed tomography (CT) on admission and after 24 hours. Evaluation required by neurological Telemedicine Hospital Israelita Albert Einstein shortly after the conclusion of the TC Cranio.

\section{Results:}

Alteplase was used in 64 patients with diagnosis of stroke ischemic. Evaluation Neurology Telemedicine trough has been triggered in 51 cases. Time between onset of symptoms and drug administration, 19 patients less than 90 minutes, 37 patients between 90 and 180 minutes, 8 patients $180-360$ minutes .The average NIHSS at admission was 18, with 42 patients showed a reduction of 5 or more the points NIHSS score within the first 24 hours. 8 patient non-symptomatic intracranial hemorrhage and 6 symptomatic intracranial hemorrhage and 9 deaths during the period.

\section{Conclusions:}

All patients receiving alteplase in the recommended time interval and underwent CT Cranio control. Some cases were not triggered by the evaluation of Neurology Telemedicine. There was improvement in NIHSS score similar percentage observed in reference studies. The protocol implementation has been adequate excellent support of Telemdicina Neurology team. Good profitability of time and therapeutic efficacy. The mortality that correlated with the severity of patients and the NIHSS admission. This data ratifies the safety of intravenous Alteplase use in the treatment of Ischaemic Stroke. 


\title{
Poster 102
}

\section{Evaluation of Fibrinogen Concentrate (Riastap) for the Treatment of Post-tPA Hemorrhage}

\author{
Megan E. Barra, Sarah S. Culbreth, Katelyn K. Sylvester, Eva E. Rybak.
}

Department of Pharmacy, Brigham and Women's Hospital, Boston, MA, USA.

\section{Introduction:}

In patients who receive tPA for the treatment of ischemic stroke, the risk of secondary symptomatic intracerebral hemorrhage $(\mathrm{ICH})$ is approximately $6 \%$ and non- $\mathrm{ICH}$ bleeding over $5 \%$. Hypofibrinogenemia occurs in approximately $5 \%$ of tPA-treated patients and is associated with an increased risk of bleeding. Cryoprecipitate is often used to restore fibrinogen levels, despite limited published evidence.

Cryoprecipitate has several limitations, including the need for ABO matching, thawing, and concerns regarding potential transmission of viral pathogens. Riastap, a purified fibrinogen concentrate, is a promising alternative to cryoprecipitate for the reversal of hemorrhage post-tPA. The objective of this study was to evaluate the safety and efficacy of Riastap for the treatment of post-tPA hemorrhage.

\section{Methods:}

A single-center retrospective observational analysis was conducted to evaluate patients who received Riastap for the treatment of post-tPA hemorrhage between 1/1/2012 and 12/31/2015. The main outcome measure was reversal of hypofibrinogenemia. Hypofibrinogenemia was defined as a fibrinogen level < $200 \mathrm{mg} / \mathrm{dL}$. Secondary outcomes include incidence of thrombotic events, infusion reactions, and inhospital mortality.

\section{Results:}

Eleven patients were included in our analysis. The average dose of Riastap administered was 3,443 units. Five patients had hypofibrinogenemia prior to Riastap administration, with a mean fibrinogen level of $97.8 \mathrm{mg} / \mathrm{dL}$. The median increase in fibrinogen levels in hypofibrinogenemic patients was $143 \mathrm{mg} / \mathrm{dL}$ (IQR 123 to 150). The six patients who were not hypofibrinogenemic at baseline had minimal effect on fibrinogen levels post-Riastap administration, with a median change in fibrinogen levels of $17 \mathrm{mg} / \mathrm{dL}$ (IQR -14.8 to 57 ). One patient was diagnosed with a deep vein thrombosis 10 days post-Riastap administration and no infusion reactions were reported. In-hospital mortality occurred in $45.5 \%$ of our patient population.

\section{Conclusions:}

Riastap administration successfully and safely treated hypofibrinogenemia in patients with post-tPA hemorrhage. 
Poster 103

\title{
Impact of Implementing Team-Driven Interventions to Reduce Catheter Associated Urinary Tract Infections in the Neuroscience Intensive Care Unit
}

\author{
Casey Olm-Shipman 1 , Megan M. Brisse ${ }^{2}$, Marin M. Darsie ${ }^{2}$, Rhonda R. Cadena ${ }^{2}$, Sharmila S. Soares- \\ Sardinha ${ }^{2}$, Christa C. Williams ${ }^{2}$, Jeff J. Laux ${ }^{3}$, Kevin K. Robertson ${ }^{2}$, Dedrick D. Jordan ${ }^{2}$. \\ ${ }^{1}$ University of North Carolina at Chapel Hill School of Medicine, Department of Neurology, Chapel Hill, \\ NC, USA, ${ }^{2}$ University of North Carolina at Chapel Hill, Department of Neurology, Chapel Hill, USA, ${ }^{3}$ \\ University of North Carolina at Chapel Hill, School of Public Health, Chapel Hill, USA.
}

\section{Introduction:}

Catheter-associated urinary tract infections (CAUTI) are the most prevalent hospital-acquired infections (HAI), and account for more than 560,000 cases annually and 13,000 deaths per year. CAUTI is the most common HAI in Neuroscience Intensive Care Units (NSICU), due to risk factors including patient immobility and urinary retention. We implemented a team-driven multimodal quality improvement initiative to reduce CAUTI and catheter-utilization rates in the NSICU.

\section{Methods:}

We convened a multidisciplinary CAUTI prevention team including nurses, advance practice providers, physicians, and infection control specialists. We developed a CAUTI surveillance program that involved dissemination of CAUTI reports to stakeholders, as well as monthly team meetings to review CAUTI and catheter utilization rates. We applied root cause analysis to target improvement opportunities, and implemented interventions including best-practice catheter insertion techniques, modification of bowel regimen, and guidelines for timing of catheter removal. We also implemented a daily goals tool to prompt standardized team communication surrounding catheter removal on morning interdisciplinary rounds. We performed Poisson generalized linear model analyses, controlling for linear time trends and testing with sandwich errors.

\section{Results:}

We analyzed data before and after implementation of interventions, spanning a time period of 28 months. Mean CAUTIs per month declined $(2.13+/-1.3$ vs 0.5 to $0.5+/-0.58)$, as did mean catheter days per month $(329+/-31.2$ vs $267+/-33.2)$. We observed a $68 \%$ reduction in CAUTI rate adjusted by catheter days $(95 \% \mathrm{Cl} 6 \%, 89 \%), p=0.035$. There was an $11 \%$ reduction in catheter utilization rate adjusted by patient days $(95 \% \mathrm{Cl} 3 \%, 19 \%), \mathrm{p}=0.011$.

\section{Conclusions:}

A team-driven multimodal approach to CAUTI reduction resulted in significant decreases in CAUTI and catheter utilization rates in the NSICU. Team-driven interventions enhance communication and shared stakeholder accountability. Follow-up is ongoing to evaluate sustainability. 


\title{
Poster 104
}

\section{Non-Neurological Complications In Traumatic Neurosurgical ICU Patients: A Prospective Observational Study}

\author{
Keshav Goyal', Amarjyoti A. Hazarika ${ }^{2}$, Navdeep N. Sokhal $^{3}$, shweta s. Kedia ${ }^{4}$, Parmod P. Bithal ${ }^{5}$. \\ ${ }^{1}$ Critical and intensive care, Neuroanaesthesiology, JPNATC, AIIMS, New Delhi, India, ${ }^{2}$ Department of \\ Anaesthesiology, PGIMER, Chandigarh, India, Chandigarh, India, ${ }^{3} \mathrm{Critical}$ and intensive care, \\ Neuroanaesthesiology, JPNATC,AIIMS, New Delhi, India, ${ }^{4}$ Neurosurgery, All India institute of Medical \\ Sciences, New Delhi, India, ${ }^{5}$ Neuroanaesthesiology, All India Institute of Medical Sciences, New Delhi, \\ India.
}

\section{Introduction:}

Non-neurological complications involving a single or multiple organ systems during intensive care in critically ill patients of traumatic brain and spine injuries is significant cause of poor prognosis but often not well managed. The aim of this study was to assess the frequency of such complications in neuro ICU and assess their impact on morbidity and mortality.

\section{Methods:}

A prospective observational study on 200 patients of varied demographic profile admitted in neuro ICU over a period of 6 months for conservative /operative management, with traumatic brain and spinal cord injury and associated multisystem involvement was conducted. Significant predefined parameters addressing the non-neurological complications occurring during their ICU stay were recorded including respiratory, cardiovascular, Infection, acute kidney injury (AKI), gastrointestinal complications, electrolyte disturbances and bleeding complications. The study period was from admission to the ICU till the discharge from the ICU or demise.

\section{Results:}

$63 \%$ of patients developed respiratory complications in the form of chest infiltrate $(50 \%)$ and atelectasis $(10.31 \%) .35 .5 \%$ of patients suffered from cardiovascular complications. $37 \%$ of patients had dyselectrolytemia, commonest being hypernatremia due to hypovolemia (70\%). Sepsis was observed in $23.5 \%$. Bleeding diathesis and acute renal injury were observed in $22 \% \& 3.5 \%$ of patients respectively. $17 \%$ of the patients succumbed to injury out of which $64.7 \%$ was due to non -neurological cause. Further results will be discussed in detail with inferences at the meeting.

\section{Conclusions:}

Intensivists in neuro ICU must consistently assess and treat the non-neurological complications in traumatic brain and spine-injured patients and deliver appropriate care to bring down the mortality and morbidity and improve outcome. 


\title{
Poster 105
}

\section{Transcranial Doppler in Patients Treated with Extracorporeal Membrane Oxygenation: Changes in Pulsatility Index}

\author{
Megan E. Esch ${ }^{1}$, Tapan T. Kavi ${ }^{1}$, Brenda B. Rinsky', Shouri S. Lahiri', Axel A. Rosengart ${ }^{1}$, Patrick P. \\ Lyden ${ }^{1}$. \\ ${ }^{1}$ Cedars Sinai Medical Center/Department of Neurology, Los Angeles, CA, USA, ${ }^{2}$ Cedars Sinai Medical \\ Center/Department of Neurology, Los Angeles, USA.
}

\section{Introduction:}

Transcranial Doppler (TCD) is a useful ancillary test in neurologic critical care for monitoring patients at risk of cerebral vasospasm and evaluating for elevations in intracranial pressure. Pulsatility Indices (PIs) are a known TCD parameter correlating with elevated intracranial pressure and cerebral vascular resistance. The normal values of cerebral blood flow velocity and PIs are significantly distorted by nonpulsatile blood flow, as in patients on Venoarterial Extracorporeal Membrane Oxygenation (VA-ECMO) circulation. This analysis evaluates changes in PI measurements in patients on VA-ECMO following cardiogenic shock. It is important to be aware of these changes, as they can be misinterpreted as cerebral vasodilation, vasoconstriction, increased intracranial pressures, or cerebral circulatory arrest.

\section{Methods:}

Data from 11 TCDs in 8 patients on VA-ECMO in the Cedars Sinai Medical Center Cardiac Surgical ICU were reviewed. Mean Pls were calculated for each patient using Gosling's PI formula. The values obtained were compared with Ejection Fractions (EF) obtained within 24 hours of TCD.

\section{Results:}

Pls were globally low or absent in all 11 TCDs. The non-demonstrable PI seen in one patient is from severely diminished cardiac function, resulting in complete lack of systolic upstroke. In three patients, TCDs were performed at the initiation and conclusion of VA-ECMO cannulation. The PI values for these TCDs correlated directly with changes in EFs. Also, an abrupt rise in PI to normal value was seen with placement of a Total Artificial Heart and return of pulsatile circulation.

\section{Conclusions:}

We demonstrate that patients on mechanical circulatory support demonstrate low-absent PIs on TCDs. We postulate that the lack of systolic upstroke in these patients contributes to this finding. Low PIs in these patients should not be mistaken for vasodilation or cerebral circulatory arrest. Moreover, rising PIs in patients with improving cardiac function should not be confused with elevated intracranial pressures. 


\title{
Poster 106
}

\section{Practice Patterns Of Venous Thromboembolism Prophylaxis In Underweight, Critically III Patients With Neurologic Injury}

\author{
Kevin Betthauser ${ }^{1}$, Hannah H. Pope ${ }^{1}$, Mollie M. Gowan ${ }^{1}$, Theresa T. Human ${ }^{2}$. \\ ${ }^{1}$ Barnes-Jewish Hospital, Department of Clinical Pharmacy, St. Louis, MO, USA, ${ }^{2}$ Barnes-Jewish \\ Hospital, Washington University, St. Louis, MO, USA.
}

\section{Introduction:}

Venous thromboembolism (VTE) prophylaxis in underweight patients with neurologic injury remains unaddressed by recent guidelines and primary literature. This study aimed to describe VTE prophylaxis strategies employed in this population and compare the impact of underweight and non-obese patients on thrombotic and bleeding events.

\section{Methods:}

Underweight and non-obese adults admitted to the neurology/neurosurgery intensive-care unit from September 1, 2009 to July 31, 2015 were retrospectively identified. Underweight was defined as a body mass index $(\mathrm{BMI})<18.5 \mathrm{~kg} / \mathrm{m} 2$ or weight $<50 \mathrm{~kg}$ and non-obese as a BMI $18.6-29.9 \mathrm{~kg} / \mathrm{m} 2$. Patients were excluded if they received $>1$ VTE prophylaxis regimen, had an ICU length of stay $<72$ hours, or received VTE prophylaxis for $<48$ hours. Patients were stratified to non-obese and underweight groups and subsequently matched $2: 1$, on age and diagnosis. Prophylaxis regimen, prevalence and type of thrombotic and/or bleeding events, and discharge disposition were collected.

\section{Results:}

The most common regimen in the underweight $(n=107)$ and non-obese $(n=214)$ groups was unfractionated (UFH) 5000 units subcutaneously every $12 \mathrm{hrs}(69.1 \%$ vs. $83.6 \% ; p=0.003)$. Only underweight patients received UFH 2500 units subcutaneously every $12 \mathrm{hrs}(17.8 \%$ vs. $0.0 \% ; p<0.0001)$. Non-obese and underweight patients had no difference in the proportion of overall bleeding $(5.6 \%$ vs. $10.3 \% p=0.204)$ and thrombotic events $(6.1 \%$ vs. $2.8 \% p=0.204)$ while receiving VTE prophylaxis. Further analyses revealed a statistically significant difference in the proportion of underweight patients that developed intracranial hematoma expansion while receiving prophylaxis versus non-obese patients $(45.5 \%$ vs. $8.3 \% ; p=0.017)$. A trend toward an increased risk of bleeding was seen in patients that received $>150$ units $/ \mathrm{kg} /$ day of UFH $(9.7 \%$ vs. $4.2 \%$; $p=0.064)$. No differences in discharge dispositions were seen between groups.

\section{Conclusions:}

Current practice does not reflect a consistent dose reduction for neurologically-injured, underweight patients. Caution should be considered when using increased doses of UFH in neurologically-injured patients. Continued assessment of VTE prophylaxis is needed to confirm these findings. 


\title{
Poster 107
}

\section{Safety of Phenylephrine Infusion through Peripheral Intravenous Catheter in Neuro Intensive Care Unit}

\author{
Adam Schertz ${ }^{1}$, Eddy E. Gutierrez ${ }^{2}$, Sudhir S. Datar ${ }^{3}$. \\ ${ }^{1}$ Wake Forest University Baptist Medical Center/Internal Medicine, Winston Salem, NC, USA, ${ }^{2}$ Wake \\ Forest University Baptist Medical Center/Anesthesiology, Winston Salem, NC, USA, ${ }^{3}$ Wake Forest \\ University Baptist Medical Center/Neurology, Winston Salem, NC, USA.
}

\section{Introduction:}

Patients in the neuro intensive care unit (NICU) commonly need vasopressor infusions for various reasons. The traditional approach is to insert central venous catheters (CVC) for this purpose. CVCs carry the risk of complications such as infections, local tissue injury, pneumothorax and deep vein thrombosis among others. Phenylephrine is a commonly used vasopressor in the NICU. The purpose of this study was to evaluate the safety of phenylephrine infusion through peripheral intravenous catheter (iv).

\section{Methods:}

Retrospective review of consecutive patients admitted to the Neuro ICU and administered phenylephrine infusion through peripheral iv line.

\section{Results:}

One hundred patients, mean age 66 years $(S D \pm 14)$ were included in the analysis. Fifty-four $(54 \%)$ were men. Eighty-nine $(89 \%)$ patients had at least 1 or more vascular risk factors or established cardiovascular disease. The most common indications of phenylephrine were hemodynamic augmentation (37\%), multifactorial transient post-operative hypotension (28\%) and hypotension due to other causes (22\%). Most common location of IV line was proximal upper extremity (28 \% antecubital, $28 \%$ forearm) with gauge of the IV line between 18 (45\%) and 20 (44\%). Average maximum rate of phenylephrine infusion (concentration $120 \mathrm{mcg} / \mathrm{ml}$ ) was $80 \mathrm{mcg} / \mathrm{min}$ (SD \pm 53 , range 5 to 200) and was continued for a mean duration of 19 hours (SD \pm 18 , range 1 to 114 ). Central line was eventually placed in $16 \%$ due to physician preference and in another $5 \%$ due to a change of vasopressor to norepinephrine. There were 2 confirmed and 3 potential episodes of iv infiltration and 2 episodes of iv leakage. None of the patients had any complications.

\section{Conclusions:}

Infusion of phenylephrine through peripheral iv appears safe when used in moderate doses for the short term and can be considered in lieu of placing a central line solely for this purpose. This may reduce the complications associated with central lines. 
Poster 108

\section{A Retrospective Review of Combination Low Dose Mannitol and Hypertonic Saline for the Treatment of Cerebral Edema}

Christina Roels, Peter P. Keddis, Anna A. Purvis, Benjamin B. Anyanwu.

Novant Health Forsyth Medical Center, Winston-Salem, NC, USA.

\section{Introduction:}

Osmotic therapy continues to be standard care in the medical management of cytotoxic cerebral edema. There is a lack of evidence to support preference amongst osmotic agents or the use of multiple agents. The long term use of monotherapy is often limited by side effect profile. The combination of low dose mannitol and hypertonic saline may provide synergistic effect by combining mechanisms of action, while limiting dose-related toxicities of either agent. We investigated safety and efficacy endpoints for combination therapy.

\section{Methods:}

A single-center retrospective cohort study from August 2014 to December 2015. Identified patients were administered combination mannitol and hypertonic saline for $>48$ hour duration. The primary outcome was the safety of combination therapy described by the incidence of acute kidney injury (AKI) by AKIN criteria, sodium fluctuation, and central pontine myelinolysis.

\section{Results:}

31 patients (mean age $55 \pm 14,52 \%$ male) were identified. Underlying neurological injury included $44 \%$ intracerebral hemorrhage, $38 \%$ ischemic stroke, $9 \%$ aneurysmal subarachnoid hemorrhage, $6 \%$ traumatic brain injury. $52 \%$ had neurosurgical management. The average number of mannitol doses given was 22 , and the average duration of hypertonic saline was 110 hours. The range of mannitol dose was $0.14-0.6$ $\mathrm{gm} / \mathrm{kg}$, and the most frequent hypertonic saline dose was $3 \%$ continuous infusion $30 \mathrm{~mL} / \mathrm{hr}$. A low percentage of osmotic therapy doses were held for predefined osmolality/sodium parameters $(27 \%$ held mannitol, $17 \%$ held hypertonic saline). AKI occurred in 10 (32\%) patients ( 9 - stage 1 AKI, 1 - stage 3 AKI). All kidney injuries resolved. Sodium fluctuation occurred in 3 patients (10\%). No patients developed central pontine myelinolysis.

\section{Conclusions:}

Low dose combination osmotic therapy was tolerated with no central pontine myelinolysis and rare sodium fluctuations; however transient low grade AKI was common. Further study is needed to evaluate the relative efficacy of single and combination osmotic therapy in the neurocritical care population. 


\title{
Poster 109
}

\section{Clinical characteristics of nonconvulsive status epilepticus diagnosed by simplified continuous EEG monitoring at an emergency intensive care unit.}

\author{
Satoshi Egawa', Toru T. Hifumi ${ }^{2}$, Kenya K. Kawakita ${ }^{2}$, Hidetoshi H. Nakamoto ${ }^{3}$, Yuichi Y. Kubota ${ }^{4}$, \\ Yasuhiro Y. Kuroda².
}
${ }^{1}$ Asakadai Central General Hospital. Neurosurgery, Neurocritical Care Unit., Asaka, Japan, ${ }^{2}$ Emergency Medical Center, Kagawa University Hospital, Kagawa, Japan, ${ }^{3}$ Asaka Central General Hospital I Neurosurgery, Stroke and Epilepsy Center., Saitama, Japan, ${ }^{4}$ Asaka Central General Hospital I Neurosurgery, Stroke and Epilepsy Center., Saitama, Japan.

\section{Introduction:}

The clinical characteristics of Nonconvulsive status epilepticus (NCSE) presenting in ICU in Japan is limited. Our institute provides a noninvasive monitoring system of two-channel simplified continuous EEG (sEEG) for the bedside monitoring of cerebral activities. The present study aimed to elucidate the clinical characteristics of NCSE in patients with altered mental status (AMS).

\section{Methods:}

This single-center retrospective study comprised 149 patients who were hospitalized between March 1 , 2015 and September 30, 2015 at the emergency intensive care unit (ICU) of the Kagawa University Hospital. Primary outcome was the NCSE incidence. The secondary outcome was the comparison of duration of ICU stay, hospital stay, and a favorable neurological outcome (FO), as assessed using the modified Rankin Scale (mRS) score at discharge from our hospital between the groups with and without NCSE. FO and poor neurological outcomes (PO) were defined as mRS scores of 0-2 and 3-6, respectively.

\section{Results:}

Simplified continuous electroencephalogram (sEEG) was monitored in 36 patients (median age, 68 years; $69.4 \%$ males) with acute AMS. NCSE was observed in $11(30.1 \%)$ of the 36 patients with AMS. Rates of FO, duration of ICU stay, and hospital stay were not significantly different between the NCSE and nonNCSE groups ( $p=0.45, p=0.30$, and $p=0.26$, respectively).

\section{Conclusions:}

Approximately $30 \%$ of the patients with AMS admitted to emergency ICUs developed NCSE. The outcomes of AMS patients with and without NCSE did not differ significantly when appropriate medical attention and antiepileptic drugs were initiated. sEEG monitoring may be recommended in patients with AMS in emergency ICU to obtain early detection of NCSE followed by appropriate intervention. 


\title{
Poster 110
}

\section{Which Spontaneous Breathing Trial Predicts Liberation Success In Neurocritical Care Patients?}

\author{
Naresh Mullaguri ${ }^{1}$, Premkumar P. Nattanmai ${ }^{2}$, Newey N. Christopher ${ }^{3}$. \\ ${ }^{1}$ Department of Neurology, University of Missouri-Columbia, Missouri, Columbia, MO, USA, ${ }^{2}$ Department \\ of Neurology, University of Missouri-Columbia, Columbia, MO, USA, ${ }^{3}$ Department of Neurology/University \\ of Missouri-Columbia, Columbia, MO, USA.
}

\section{Introduction:}

Approximately 200,000 people per year will need mechanical ventilation secondary to neurological injury resulting in significant mortality. Delaying liberation in neurologically impaired patients otherwise ready for liberation is a source for significant hospital charges. There is no clear guideline to suggest one spontaneous breathing trial (SBT) over another in predicting the liberation success. Zero pressure support and zero positive end expiratory pressure (PEEP) or ZEEP is a traditional method assessing patient's readiness for mechanical ventilation liberation. However, neurologically injured patients with dysregulation of autonomic function may be at a greater risk of failing ZEEP. The purpose of this study was to assess mechanical ventilation liberation in patients who failed ZEEP and subsequently passed pressure support trial.

\section{Methods:}

Retrospective analysis of liberation in 134 intubated patients in a neurosciences intensive care unit. All patients were initially challenged with ZEEP. If passed, patients were liberated from mechanical ventilation. If failed, a $5 \mathrm{cmH} 2 \mathrm{O}$ pressure support and $5 \mathrm{cmH} 2 \mathrm{O}$ PEEP (i.e., 5/5) SBT was performed. If the patient then passed $5 / 5$, the patient was liberated. Statistical analyses were performed using McNemar's exact test. $P$ value $<0.05$ was considered significant.

\section{Results:}

134 adult (>18 years old) patients were included. The majority of patients were successfully liberated from mechanical ventilation using 30 minute ZEEP trial alone $(n=123,91.7 \%)$. Eleven $(8.21 \%)$ patients failed ZEEP but immediately passed $5 / 5$ SBT trial. One patient $(0.74 \%)$ required reintubation. Ten $(7.46 \%)$ patients were successfully liberated $(p=0.0044)$. Average duration for $5 / 5$ SBT was $1.66+1.16$ hours. Tachypnea/agitation was the cause of failing ZEEP in all patients.

\section{Conclusions:}

This study shows that the majority of patients can be successfully liberated from mechanical ventilation after passing a 30 minute ZEEP trial. In those who fail, a 5/5 SBT can significantly increase the successful liberation from mechanical ventilation. 


\title{
Poster 111
}

\section{Usage of a population pharmacokinetic model of vancomycin in neurosurgical intensive care unit patients}

\author{
WANG Rong ${ }^{1}$, ZHU Z. Huaijun ${ }^{2}$. \\ ${ }^{1}$ Department of Neurosurgery, the Affiliated Drum Tower Hospital, Nanjing, China, ${ }^{2}$ Department of \\ Pharmacy, Nanjing, China.
}

\section{Introduction:}

The purpose of this study was to test the validity and accurate of a population pharmacokinetic model of vancomycin establishey using NONMEM software by the department of pharmacy of Nanjing Drum Tower Hospital in neurosurgical intensive care unit patients.

\section{Methods:}

According to the patient's gender, age, body weight, serum creatinine (SCr), serum albumin (ALB), the predictive value of vancomycin was calculated from the pharmacokinetic model and compared with the actual measured value.

\section{Results:}

During the period from March 2013 to March 2014, 42 patients including 25 male and 17 female, whose age is $53 \pm 14$ years old (22-78 years old), were grouped and 53 copies of blood concentration of vancomycin were measured. The average concentration was $10.9 \mathrm{mg} / \mathrm{L}$ and the range was $1.6 \mathrm{mg} / \mathrm{L}$ to $49.1 \mathrm{mg} / \mathrm{L}$. The pharmacokinetic prediction values from calculated mode were a significant correlation with the actual measured value $(r=0.857, P<0.001)$, the mean absolute percentage error (MAPE) was 0.4079 .

\section{Conclusions:}

the pharmacokinetic model can be used for patients with intravenous infusion of vancomycin in neurosurgical intensive care unit patients for drug value prediction and drug dosage guidance. But because of coma, the body weight estimation has errors (about $30 \%$ ). The renal function sometimes changed by contrast agent and diuretic drug has an impact on predictive results. By adjusting methods, accurate prediction rate increased to nearly $70 \%$. 
Poster 112

\section{Effect of Clevidipine and Nicardipine on Blood Pressure Variability}

Xi Liu-DeRyke, Sindhuri S. Avula, Jason J. Vilar.

Florida Hospital Orlando/Pharmacy department, Orlando, FL, USA.

\section{Introduction:}

Nicardipine is the standard of care for blood pressure (BP) management following acute strokes, whereas little data exists concerning clevidipine in this population. Large variations in BP during the first 24 hours is an independent predictor for poor outcomes in acute stroke patients. The purpose of this study was to evaluate the effect of clevidipine and nicardipine on BP variability following acute strokes.

\section{Methods:}

Adult patients with acute ischemic stroke (AIS), intracerebral hemorrhage $(\mathrm{ICH})$, or aneurysmal subarachnoid hemorrhage (aSAH) admitted to neuroscience intensive care unit from January 2012 through December 2015 were identified retrospectively. Patients were included if they received clevidipine or nicardipine for initial acute BP management, and BP goal was defined by the prescribers. $\mathrm{BP}$ variability was measured by standard deviation (SD) of mean arterial pressure (MAP) over the first 24 hour of therapy.

\section{Results:}

Seventy three patients were included in the analysis (clevidipine $n=18$; nicardipine $n=55$ ). Admission stroke types in clevidipine and nicardipine group were: $6 \%$ and $31 \%$ AIS, $83 \%$ and $44 \% \mathrm{ICH}$, and $11 \%$ and $25 \%$ aSAH. Baseline MAP between clevidipine and nicardipine group was comparable (102 vs. 109 $\mathrm{mmHg}$ ). The number of BP recordings was similar between groups (clevidipine $22 \mathrm{vs}$. nicardipine 24; $p=0.51)$ and the average time to goal was 116 minutes and 47 minutes, respectively $(p=0.15)$. The average MAP during the first 24 hours was similar (clevidipine 86 vs. nicardipine $84 \mathrm{mmHg} ; \mathrm{p}=0.26$ ). Although not statistically significant, clevidipine group had a higher percentage of BP above goal compared to nicardipine group $(24.6 \%$ vs $9.8 \% ; p=0.09)$. There was no significant difference in BP variability between clevidipine and nicardipine group (SD 11.6 vs. $13.5 \mathrm{mmHg} ; p=0.09$ ).

\section{Conclusions:}

Our study did not find a difference in BP variability between clevidipine and nicardipine following acute strokes, which may be attributable to the small sample size and retrospective design. 


\title{
Poster 113
}

\section{Prevalence of Ventricular Arrhythmias and Cardiac Arrest in patients with Prolonged QT interval in Neuro ICU}

\author{
Megan Garzon ${ }^{1}$, Sudhir S. Datar². \\ ${ }^{1}$ Wake Forest School of Medicine, Winston Salem, NC, USA, ${ }^{2}$ Wake Forest University Baptist Medical \\ Center/Neurology, Winston Salem, NC, USA.
}

\section{Introduction:}

Long corrected QT interval (QTc) has been associated with malignant ventricular arrhythmias specifically torsades de pointes (TdP) and can be due to a variety of risk factors many of which are commonly present in neuro intensive care unit (NICU) patients. In addition to medical causes, acute neurologic insult has been shown to cause multiple neuro-cardiac manifestations including QTc prolongation. Prevalence of ventricular arrhythmias in patients with abnormal QTc has never been studied in the NICU, knowledge of which is important in their medical decision making. All the currently available data comes from medical and surgical ICU patients which have different disease processes compared to NICU.

\section{Methods:}

Retrospective review of consecutive patients admitted to the Neuro ICU and having abnormal QTC interval.

\section{Results:}

Ninety-five patients, mean age 66 years (SD \pm 14 ) were included in the analysis. Fifty four $(54 \%)$ were men. Average duration of hospitalization was 12 days (SD \pm 11 ). Major causes for admission were stroke $(47 \%)$, subdural hemorrhage (13\%), and cerebral hemorrhage (12\%). Fifty-one patients $(54 \%)$ had known cardiovascular disease, $30 \%$ had abnormal ejection fraction. Thirty-seven patients $(39 \%)$ needed mechanical ventilation, 34 (36\%) were septic and 4 patients were in septic shock. Multiple electrolyte abnormalities were observed throughout the hospitalization and patients frequently received QTC prolonging drugs. Mean QTc was $495 \mathrm{~ms}$ (SD \pm 37 , range 450-659). There were 6 episodes (6\%) of nonsustained ventricular tachycardia which did not lead to any immediate consequences. One patient had cardiac arrest following anesthesia for hemicraniectomy. Initial rhythm was asystole followed by fine ventricular fibrillation and therefore could not be clearly attributed to prolonged QTc. There were no episodes of TdP.

\section{Conclusions:}

In this group of selected patients at high risk of ventricular arrhythmias, occasional non-sustained ventricular tachycardia was observed without leading to cardiac arrest. No episodes of TdP were observed in these patients. 


\title{
Poster 114
}

\section{Prevalence of deep vein thrombosis among neuro-critical intensive care unit patients}

\author{
Amandeep S. Dolla ${ }^{1},{ }^{2}$, Navid N. Tabibzadeh ${ }^{1}$, Amna A. Sheikh ${ }^{1}$, Ilya I. Levin ${ }^{1}$, Rodney R. Bell ${ }^{3}$, Matthew \\ M. Vibbert ${ }^{4}$. \\ ${ }^{1}$ Thomas Jefferson Hospital/Dept of Neurology, Philadelphia, PA, USA, ${ }^{2}\left[\left[\left[N O T\right.\right.\right.$ FOUND]]], ${ }^{3}$ Thomas \\ Jefferson hospital/Dept of Neurology, Phialdelphia, PA, USA, ${ }^{4}$ Thomas Jefferson hospital/Dept of \\ Neurological surgery, Phialdelphia, PA, USA.
}

\section{Introduction:}

Deep venous thrombosis (DVT) of the lower extremities is a common cause of morbidity and mortality among neurologically injured patients. The data on incidence and prevalence rates of DVT among high risk neurologic populations is scarce. The available literature focuses largely on patients admitted to a medical or surgical intensive care unit with very limited information on patients in neuro-intensive care units (NICU). The aim of the present study is to assess the incidence and prevalence of deep vein thrombosis among patients admitted with acute neurologic injury.

\section{Methods:}

Our institution routinely conducts ultrasound screening within 24 hours of admission and weekly thereafter in high risk neurologically injured patients. We conducted a retrospective review of ultrasound records of 2,644 patients admitted to NICU, stroke or INICU at a university hospital over a 13-month period. Data was abstracted and analyzed to assess the prevalence of DVT in this period. We excluded patients presenting with superficial vein thrombosis, hematoma and chronic venous scarring.

\section{Results:}

Over a period of one year; the prevalence of DVT was $6.1 \%(n=161)$. Of the 161 cases that were diagnosed with DVT; more than one-half (54.7\%) presented with DVT at the time of admission. 73 patients $(45.3 \%)$ acquired DVT during hospitalization. Majority of the patients with DVT at the time of admission are Caucasian males with mean age 72 and mean SAPS II score of 34.2, ranging between 13 and 71 .

\section{Conclusions:}

Prevalence of DVT at the time of presentation to the neuro ICU is relatively high. Further research is needed to understand the risk profile of patients with acute neurological injury. 


\title{
Poster 115
}

\section{Prolonged Prophylactic Antibiotics with Neurosurgical Drains and Devices: Are we using them? Do we need them?}

\author{
Ariane Lewis, Barry B. Czeisler, Aaron A. Lord.
}

NYU Langone Medical Center, Departments of Neurology and Neurosurgery, New York, NY, USA.

\section{Introduction:}

Practice guidelines recommend that practitioners should not prescribe prolonged prophylactic systemic antibiotics (PPSA) after neurosurgical procedures, even if drains are left in place. We sought to evaluate 1) current practice patterns related to PPSA administration to neurosurgical patients with drains and devices and 2) practitioner perception about the need for PPSA in this population.

\section{Methods:}

We surveyed members of the Neurocritical Care Society on use of PPSA (defined as maintenance antibiotics after the time of insertion) and personal perception about the need for PPSA in patients with intraparenchymal monitors, subdural drains, subgaleal drains, Jackson-Pratt spinal drains, and lumbar drains.

\section{Results:}

Of 52 respondents, routine institutional use of PPSA was reported by $29-52 \%$ for each drain/device. The fewest respondents reported use with subgaleal drains and the most respondents reported use with Jackson-Pratt spinal drains with instrumentation. Respondents had varying personal opinions on the need for PPSA with each drain/device. Only fifteen respondents strongly disagreed/disagreed with the need for PPSA for every drain/device. The highest percentage of respondents who agreed/strongly agreed with the need for PPSA (35\%) for a given drain/device was for patients with spinal drains with instrumentation while the lowest (19\%) was for patients with subgaleal drains.

\section{Conclusions:}

It is clear that adherence to, and knowledge of, practice guidelines varies. Because antibiotic use is associated with risk of nosocomial infections and growth of resistant bacteria, education about guidelines on the use of PPSA in patients with neurosurgical drains is necessary to optimize patient care. 


\title{
Poster 116
}

\section{Transition to Comfort Measures Only In Critically III Patients: Survey Results from Health-Care Providers in a Large Academic Medical Center}

\author{
Abhijit Lele ${ }^{1}$, Larry L. Healey ${ }^{2}$, Sherry S. Cheever ${ }^{2}$, JoAnne J. Whitney ${ }^{3}$, Abdou A. Abdelhak ${ }^{4}$, Kellie K. \\ Carpenter ${ }^{2}$.
}

\footnotetext{
${ }^{1}$ Department of Anesthesiology \& Pain Medicine, Harborview Medical Center, Seattle, WA, USA, ${ }^{2}$ Neurocritical Care, Harborview Medical Center, Seattle, WA, USA, ${ }^{3}$ University of Washington School of Nursing, Seattle, WA, USA, ${ }^{4}$ Harborview Medical Center, Seattle, WA, USA.
}

\section{Introduction:}

Transition to comfort measures only (CMO) in an intensive care unit (ICU) is a common but delicate process that requires a well-organized multi-disciplinary and multi-professional care model. The goal of this survey was to understand potential deficiencies and inconsistencies in the transition to CMO in order to develop a process to improve the quality of care provided to patients at their end-of-life.

\section{Methods:}

After obtaining IRB approval, a web-based questionnaire was distributed to attending physicians, residents, fellow trainees, bedside nurses, respiratory therapists and spiritual care team members, who deliver care to patients in neurological, medical, trauma-surgical, and burn intensive care units at the University of Washington's Harborview Medical Center.

\section{Results:}

Overall survey response was $46.2 \%$ (297 out of 725 ). The concept that transition to CMO is a multidisciplinary process was not universal with only $9.5 \%$ of all bedside nursing and respiratory therapists feeling invited and actively engaged in the discussion about CMO. The majority of respondents $(75 \%)$ encountered at least one 'less than ideal' transition to CMO. Deficiencies identified included gaps in communication $(79.9 \%)$, gaps in knowledge (45.6\%), missed opportunities $(38.1 \%)$, and interprofessional conflict $(6.5 \%)$. Most participants $(75 \%)$ agreed that a formalized process might reduce inconsistencies in $\mathrm{CMO}$ transitions. Survey respondents $(41.8 \%)$ acknowledged that the lack of follow up with the patients might bias decision making around the transition to $\mathrm{CMO}$.

\section{Conclusions:}

We identified several barriers towards an optimal, collaborative transition to CMO in ICUs at a large academic medical center, highlighting the need for a formalized process. Such a process would ensure communication between various disciplines and professions, and offer healthcare providers opportunities for dialogue to address all the issues resulting in a smooth transition to $\mathrm{CMO}$. 


\title{
Poster 117
}

\section{Bradycardia with Dexmedetomidine in Neurocritically III Patients}

\author{
Sarah M. Adriance ${ }^{1}$, Jessica J. Biedny ${ }^{1}$, Anthony A. Gerlach ${ }^{1}$, Vanessa V. Olcese ${ }^{2},{ }^{3}$. \\ ${ }^{1}$ The Ohio State University Wexner Medical Center, Columbus, OH, USA, ${ }^{2}$ The Ohio State University \\ Wexner Medical Center, Columbus, OH, Afghanistan, ${ }^{3}[[[N O T$ FOUND]]].
}

\section{Introduction:}

Dexmedetomidine's propensity to cause bradycardia is well documented in non-neurocritically ill patients. Data show an incidence of $17 \%$ in medical/surgical intensive care units (ICU) when defined as heart rate $(\mathrm{HR})<60 \mathrm{bpm}$. Neurocritically ill patients have been excluded from all randomized trials. The aim of this study is to assess the development of bradycardia in patients with neurologic injury who have received dexmedetomidine for sedation in the ICU.

\section{Methods:}

Data were collected retrospectively on adult patients from 10/2011-9/2015. Dexmedetomidine titration was done via nursing driven protocol with no loading doses. Primary outcome was the incidence of bradycardia $(\mathrm{HR}<60 \mathrm{bpm})$ during first administration. Secondary outcomes were percent decrease in HR from baseline and time to event analysis using Cox regression. Mortality in the ICU was collected.

\section{Results:}

A total of 202 patients were included ( $61 \%$ male, mean age 55 years, mean SAPS II 42). The most common injuries were hemorrhagic stroke $(25 \%)$, traumatic brain injury $(13 \%)$ and ischemic stroke $(12 \%)$. Bradycardia occurred in 80 patients (39.6\%). The average maximum dose was higher in patients who became bradycardic $(0.82 \pm 0.37 \mathrm{mcg} / \mathrm{kg} / \mathrm{hr}$ vs. $0.67 \pm 0.37 \mathrm{mcg} / \mathrm{kg} / \mathrm{hr}, \mathrm{P}=0.005)$ while initial dose and infusion duration did not vary. Baseline HR was lower in bradycardic patients ( $87 \pm 21 \mathrm{bpm}$ vs. $96 \pm 17 \mathrm{bpm}$, $\mathrm{P}=0.001)$ and a larger mean percent decrease in baseline HR was observed $(32.8 \% \pm 15.7$ vs. $3.5 \%$ \pm 20.8 ). Median time to first bradycardic event was 11 hours [7.5-15.0] which was significantly impacted by baseline HR (hazard ratio $0.83 ; 95 \% \mathrm{Cl}, 0.72-0.95 ; \mathrm{P}=0.006$ ). Mortality was significantly lower in patients who developed bradycardia, 3.75 vs. $14.7 \%(P=0.017)$.

\section{Conclusions:}

These data indicate that bradycardia associated with dexmedetomidine occurs considerably among the neurocritically ill. Future assessment of clinically significant bradycardia and risk factors for its development would further contribute to the limited data of dexmedetomidine use within this patient population. 


\title{
Poster 118
}

\section{Medical complications after subarachnoid hemorrhage.}

\author{
Gisele Silva ${ }^{1}$, Renata R. Miranda ${ }^{1}$, Rodrigo R. Massaud ${ }^{2}$, Andreia A. Vacari ${ }^{1}$, Guilherme G. Schettino ${ }^{3}$, \\ Miguel M. Cendoroglo Neto ${ }^{4}$.
}

${ }_{3}^{1}$ Hospital Israelita Albert Einstein, São Paulo, Brazil, ${ }^{2}$ Hospital Isarelita Albert Einstein, São Paulo, Brazil, ${ }^{3}$ Hospital Israeilita Albert Einstein, São Paulo, Brazil, ${ }^{4}$ Hospital Israeliat Albert Einstein, São Paulo, Brazil.

\section{Introduction:}

Medical complications occur frequently after subarachnoid hemorrhage (SAH). Their impact on outcome has been previously described, but was not validated in international series of SAH.

\section{Methods:}

We evaluated 139 consecutive patients admitted to a tertiary hospital in Brazil with SAH from January 2009 to May 2016. Poor outcome was defined as death or severe disability (modified Rankin score, 4-6) at discharge. We calculated the frequency of medical complications according to prespecified criteria and evaluated their impact on outcome, using multiple logistic regression after adjusting for known predictors of poor outcome.

\section{Results:}

Thirty-six $\%$ had a poor outcome; mortality was $12.6 \%$. The most frequent complications were hyperglycemia (37\%), fever (14\%), pneumonia (1.4\%), hypotension ( $<90 \mathrm{~mm} \mathrm{Hg}$ systolic) treated with vasopressors (3.6\%) and venous thromboembolism (1.5\%). Hyperglycemia (odds ratio [OR], 9.0; 95\% confidence interval [Cl], 1.7-47.8; $p=0.01$ ) significantly predicted poor outcome after adjustment for age and Hunt-Hess grade.

\section{Conclusions:}

Hyperglycemia affected more than one third of patients with SAH and was significantly associated with poor functional outcome. Critical care strategies directed at maintaining normoglycemia may improve outcome after SAH. 


\title{
Poster 119
}

\section{Exploring Associations between Invasive and Non-Invasive Blood Pressure Monitoring in Patients Receiving Vasoactive Medication Infusions}

\author{
Ali A. Saherwala, Ali A. Saherwala, Sonja S. Stutzman, Junaid J. Kalia, Stephen S. Figueroa, Venkatesh \\ V. Aiyagari, DaiWai D. Olson.
}

UT Southwestern Medical Center, Dept. of Neurology and Neurotherapeutics, Dallas, TX, USA.

\section{Introduction:}

Blood pressure (BP) can be measured in critically-ill patients using non-invasive (oscillometric) blood pressure (NIBP) and intra-arterial blood pressure (IABP) monitoring. The accuracy of NIBP compared to the "gold standard," AIBP, has been questioned. NIBP monitors generally tend to over-read at low values and under-read at high values compared to IABP. Previous studies exploring NIBP-IABP correlations have generally been performed on patients not receiving continuous infusions of vasoactive medications. Since many critically-ill patients receive vasopressors and antihypertensive agents, we wanted to study the relationship between simultaneously-measured NIBP and IABP recordings in this patient population.

\section{Methods:}

We prospectively identified patients $(\mathrm{N}=25$, target $\mathrm{N}=70)$ admitted to a Neurosciences ICU, who had simultaneous IABP and NIBP monitoring while receiving intravenous infusions of vasopressors/antihypertensive agents. Following informed consent, paired NIBP/IAPB observations were manually abstracted via retrospective chart audit. Covariate and demographic variables were also abstracted and entered into an electronic spreadsheet. Statistical analysis performed using SAS v9.4.

\section{Results:}

Initial results from 25 subjects (60\% Caucasian, $56 \%$ male, mean age 60.3 years, mean BMI 30.3 ), received vasopressors $(n=13)$ or antihypertensive agents $(n=12)$, with 857 paired NIBP/IABP observations. Independent-samples t-tests showed a significant difference between NIBP vs IABP readings: ([SBP: $m=125$ vs $130 \mathrm{mmHg}$ respectively; $p 50 \mathrm{mmHg}(1.7 \%)]$. Bland-Altman plots demonstrated good inter-method agreement between NIBP-IABP measures (when visually excluding outliers) and demonstrated marked NIBP-AIBP SBP differences at higher blood pressures.

\section{Conclusions:}

Preliminary analysis indicates a statistically significant difference between NIBP-IABP readings for patients on vasoactive medications. Yet when visually excluding outliers, there is good inter-method agreement. Data from the entire cohort will be available for presentation at the NCS annual meeting and will be helpful in choosing appropriate BP monitoring methods for patients on vasoactive infusions. 
Poster 120

\title{
Trend of New-onset Refractory Status Epilepticus (NORSE) in Japan
}

\author{
Satoshi Egawa ${ }^{1}$, Hidetoshi H. Nakamoto ${ }^{2}$, Yuichi Y. Kubota ${ }^{3}$. \\ ${ }^{1}$ Asakadai Central General Hospital / Neurosurgery, Neurocritical Care Unit., Asaka, Japan, ${ }^{2}$ Asakadai \\ Central General Hospita.I Neurosurgery, Stroke and Epilepsy Center., Asaka, Japan, ${ }^{3}$ Asakadai Central \\ General Hospital . Neurosurgery, Stroke and Epilepsy Center., Asaka, Japan.
}

\section{Introduction:}

New-onset refractory status epilepticus (NORSE) is an important syndrome often associated with a poor outcome. The aim of the present study was to review NORSE cases in our hospital and to determine the main factor that may improve patient outcomes.

\section{Methods:}

We retrospectively reviewed our hospital medical records and database of electroencephalograms (EEGs) over a 3 years period (May 2013-May 2016). In our facility, we performed 24-h continuous EEG monitoring using the international 10-20 system. Of the monitored patients, we excluded those who were not examined emergent and in whom etiology was found to be stroke, traumatic brain injury, bacterial meningitis, herpes encephalitis, and history of epilepsy. We discussed their causes and neurological outcomes at discharge, assessed using a modified Ranking Scale (mRS). A good neurological outcome (GO) was defined as a $\mathrm{mRS}$ score of $0-2$, whereas a poor neurological outcome (PO) was defined as a score of 3-6. Moreover, we attempted to determine the main factor that influenced the neurological outcomes.

\section{Results:}

We identified 785 patients who had undergone EEG, and identified six NORSE patients among them. The average patient age was 67 years, and $50 \%(3 / 6)$ of them were male. The average Glasgow Coma Scale on arrival was 9 . All patients were diagnosed with limbic encephalitis and all had nonconvulsive status epilepticus; $67 \%(4 / 6)$ and 33\% (2/6) patients were defined as having GO and PO, respectively. Definitive treatments, such as steroids, were delayed in all PO patients.

\section{Conclusions:}

On the basis of our data, the cause of all NORSE cases was limbic encephalitis. In PO patients, definitive treatments, such as pulse steroid therapy, were delayed. This is a relatively small study. Further research is needed to identify the factors which could improve outcomes. 


\title{
Poster 121
}

\section{Intraventricular Colistin Use in Neurocritically III Patients with Meningitis/Ventriculitis}

\author{
Christopher Morrison ${ }^{1}$, Brian B. Gilbert ${ }^{2}$. \\ ${ }^{1}$ Jackson Memorial Hospital/ Clinical Pharmacy Services, Miami, FL, USA, ${ }^{2}$ Jackson Memorial \\ Hospital/Clinical Pharmacy Services, Miami, FL, USA.
}

\section{Introduction:}

Multi-drug resistant organisms (MDRO) are an increasing concern in health systems. Pathogens such as Pseudomonas aeruginosa, Acinetobacter baumanii, and carbapenamase-producing Enterobacteriaceae hold highest mortality rates especially when the central nervous system is involved. When MDROs are cultured treatment options are becoming limited and reliance on medications such as colistin and aminoglycosides is becoming more prevalent. However, penetration of these therapies into the central nervous system is concerning therefore local administration is a potential concomitant therapy.

\section{Methods:}

This study was a retrospective chart review from 2009 to 2015 for all patients with documented MDROs who received intraventricular colistin.

\section{Results:}

Seven patients from 2009 to 2015 met inclusion criteria. The average age of the patients included was 49 years old, 4 were males, and the median length of intensive care unit stay was 30 days. The dose of colistin used for each patient was $10 \mathrm{mg}$ via intraventricular route. The duration of therapy ranged from 2 14 days and all cerebrospinal fluid cultures were sterile at 7 days after administration of colistin. Each patient received concomitant systemic antibiotics while receiving intraventricular colistin. Six of the seven patients were discharged from the unit and hospital with one being discharged to a skilled nursing facility. The use of intraventricular colistin was not associated with any reported adverse events.

\section{Conclusions:}

The use of intraventricular colistin was associated with positive clinical outcomes with no reported adverse effects. 
Poster 122

\section{Myasthenic Crisis: epidemiology, economics and opportunities for change - a single center retrospective analysis.}

Avinash B. Kumar, Vikram V. Tiwari, Kevin K. Scharfman, Justin J. Calabrace.

Vanderbilt University Medical Center, Nashville, TN, USA.

\section{Introduction:}

Myasthenia gravis (MG) patients are admitted to the ICU for myasthenic crises characterized by worsening muscle weakness and impending respiratory failure. The mainstay therapy is intravenous immunoglobulin (IVIg) or plasmapheresis and supportive care. We seek to review the management, costs and care flow maps of patients admitted to our institution

\section{Methods:}

This is an IRB-approved, retrospective cohort study of patients admitted to a tertiary Neuro ICU. We included adult (age > 18 years), with a diagnosis of MG who received plasmapheresis or IVIg therapy. The demographics and clinical data were summarized for patients in the IVIg and Plasmapheresis cohorts. We also compared the ICU and hospital LOS and in addition the hospital cost data for patients in both cohorts.

\section{Results:}

The final cohort included 153 hospital encounters for 82 individual patients (46 Female) admitted between 2006-14. The mean age on admission was $56.9 \pm 19.7 \mathrm{Y}$. There was no significant difference between cohorts. The mean hospital LOS was $10.87 \pm 2.6$ d. 41/152 (26\%) encounters required mechanical ventilation; the median duration of MV was $7.5 \mathrm{~d}$ (range 1-34). The median readmission rate was $1.5 \pm 0.5$. ). 13 patients had multiple crisis readmissions $(>2)$. This cohort was socially challenged ( 5 divorced, 8 single patients). $79 \%$ (121/152) patients were discharged home with home care. Complete financial analysis included 33 patients (16 in IVIg cohort and 17 in plasmapheresis cohort). The mean hospital costs (variable direct-technical) in IVIg cohort was approx. $\$ 5000$ more than the plasmapheresis cohort. There was no statistically significant difference between in the limited financial analysis. $56.25 \%$ of patients were either medicare or Medicaid patients, $21.25 \%$ were private insurance like BCBS.

\section{Conclusions:}

The disease burden on patients and hospitals of this orphan condition are significant and continues beyond the ICU. Evidence based care pathways need to be explored for the management of this high resource utility disease. 


\title{
Poster 123
}

\section{Black tar heroin skin popping as a cause of botulism}

\author{
Salvador Cruz-Flores, Ihtesham I. Qureshi, Darine D. Kassar, Paisith P. Piriyawat, Alberto A. Maud, \\ Gustavo G. Rodriguez.
}

Texas Tech University Health Sciences Center El Paso. Department of Neurology, El Paso, TX, USA.

\section{Introduction:}

Botulism is a rare potentially fatal and treatable disorder caused by a bacterial-produced toxin that affects the presynaptic synaptic membrane resulting in a characteristic neuromuscular dysfunction. It is caused by either the ingestion of the toxin or the bacteria, inhalation, or wound infection. We present our observations with a descriptive case series of botulism secondary to black tar heroin skin popping.

\section{Methods:}

We report 15 consecutive cases of botulism presenting to University Medical Center of El Paso. Medical records where reviewed to obtain demographic information, clinical presentation, treatment and outcome.

\section{Results:}

We identified fifteen patients with mean age of 47 years, twelve men. All had administered black tar heroin through skin popping and had abscesses in the administration areas. By history the most common symptoms were dysphagia $66 \%$, weakness $60 \%$, dysarthria $53 \%$, double vision $40 \%$, blurred vision $33 \%$, and dry mouth $20 \%$. On exam the most common features were: Limb weakness $73 \%$, ophthalmoplegia $53 \%$, ptosis $46 \%$. Interestingly enough, in those patients with the documentation the pupils were reactive in $46 \%$. All patients required mechanical ventilation and all were treated with the trivalent antitoxin. Thirteen patients were discharged home and 2 were transferred to a skill nursing facility.

\section{Conclusions:}

In our patients, black tar heroin skin popping, the action of injecting under the skin acetylated morphine derivatives (mostly 6-monoacetylmorphine and 3-monoacetylmorphine) was associated with the development of botulism. Its presence in the US-Mexican border is not surprising since is frequently produced in Latin America. Its association with the development of botulism should be recognized early to allow a prompt diagnosis and treatment with the antitoxin. A clinical feature worth noting is the presence of normal pupillary light reflex in nearly half of patients thus a normal pupillary response should not be used as a finding to exclude botulism. 


\title{
Poster 124
}

\section{African American Enrollment in CLEAR III}

\author{
Karen Lane ${ }^{1}$, Steven S. Mayo ${ }^{2}$, Sarah S. Lenington ${ }^{3}$, Richard R. Thompson ${ }^{4}$, Nichol N. McBee ${ }^{2}$, Wendy \\ W. Ziai ${ }^{2}$, Scott S. Janis ${ }^{5}$, Daniel D. Hanley ${ }^{2}$.
}

${ }^{1}$ Johns Hopkins University/Neurology, Baltimore, MD, USA, ${ }^{2}$ Johns Hopkins University / Neurology, Baltimore, MD, USA, ${ }^{3}$ Emissary International, Austin, TX, USA, ${ }^{4}$ Johns Hopkins Bloomberg School of Public Health / Biostatistics, Baltimore, MD, USA, ${ }^{5}$ NIH / NINDS, Baltimore, MD, USA.

\section{Introduction:}

Lack of diversity in clinical trials limits the discovery of effects that may be particularly relevant to underrepresented populations. CLEAR III, a 500-patient RCT evaluating alteplase in hemorrhagic stroke, presented an opportunity to evaluate African American (AA) enrollment.

\section{Methods:}

Investigators across 61 U.S. hospitals screened 8,587 patients over a 5-year period: $25 \%$ AA; $4.3 \%$ Asian; $0.5 \%$ Native American; $0.3 \%$ Pacific Islander; $69.7 \%$ White; $0.2 \%$ mixed race; and $10.8 \%$ not reporting. The mean age for AAs was younger at 57.9 (SD: 0.6) vs. 65.7 (SD: 0.4) for Whites ( $p=0.001$ ).

\section{Results:}

The randomized-to-screened ratio for AAs was 8.7 vs. $3.5 \%$ for other racial groups $(p<0.001)$. Higher success with AA screening was similar when broken down by U.S. census region: Midwest $(10.3 \%$ vs. $3.6 \%, p=0.26)$; Northeast $(7.8 \%$ vs. $2.9 \%, p<0.001)$; South $(8.2 \%$ vs. $4 \%, p=0.003)$; and West $(8.9 \%$ vs. $3.8 \%, p=$ nonsignificant). African Americans were less frequently excluded due to non-hypertensive etiology $(16.9 \%$ vs. $26 \%$, $p<0.001)$, not having ventricular drainage $(22.1 \%$ vs. $25.4 \%, p=0.031)$, DNR status $(18.4 \%$ vs. $25.2 \%, p=0.027)$ and unstable bleeding $(13.9 \%$ vs. $25.1 \%, p=0.024)$; and more frequently excluded for prior disability ( $32 \%$ vs. $24.9 \%, p=0.23)$, larger hemorrhages $(26.7 \%$ vs. $24.8 \%$, $p=0.44)$, and by investigator decision ( $46.5 \%$ vs. $24.9 \%, p<0.001)$. Of the 119 patients who refused consent, AAs accounted for $44.1 \%$ vs. $49.5 \%$ of Whites. In an unadjusted logistic model, the odds ratio for successful enrollment of AAs was $2.74(p<0.001)$ vs. Whites, and $2.45(p<0.001)$ after adjustment for age and Hispanic ethnicity. The age $<49,50-59$ and $60-69$ subgroups maintained higher adjusted odds ratios than Whites at $2.60(p<0.001), 2.20(p<0.001)$ and $2.43(p<0.001)$ respectively; the above 70 subgroup was not significantly different.

\section{Conclusions:}

Others have reported difficulty enrolling AAs into clinical trials. CLEAR III suggests this may be a misperception in the context of hemorrhagic stroke. 


\title{
Poster 125
}

\section{Dynamic quantitative EEG signatures predict outcome in cardiac arrest}

\author{
Edilberto Amorim ${ }^{1}$, Mohammad M. Ghassemi ${ }^{2}$, Jong J. Lee ${ }^{3}$, M. Brandon M. Westover ${ }^{1}$. \\ ${ }^{1}$ Massachusetts General Hospital, Boston, MA, USA, ${ }^{2}$ Massachusetts Institute of Technology, Cambridge, \\ MA, USA, ${ }^{3}$ Brigham and Womens' Hospital, Boston, MA, USA.
}

\section{Introduction:}

Current prognostication strategies in cardiac arrest do not take advantage of the full potential of continuous measurement of neural networks' function provided by EEG monitoring. We developed a pipeline that utilizes machine-learning algorithms to integrate clinical data and quantitative EEG (QEEG) trends, providing continuous estimation of prognosis.

\section{Methods:}

A collaboration involving two academic centers in the U.S. assembled a retrospective clinical and EEG database of adult subjects with cardiac arrest and return of spontaneous circulation who underwent continuous EEG monitoring. Four QEEG features were included in the model: regularity, Tsalis entropy, alpha-to-delta ratio, and voltage $<20 \mathrm{uV}$. Only the first 48 hours of EEG data were evaluated in this analysis. Poor outcome was defined as Cerebral Performance Category of 3-5 at discharge. Ten fold cross validation resampling method was utilized, and model performance evaluation metrics were area under ROC curve (AUC), sensitivity, and specificity. The algorithm provided an hourly estimation of poor outcome likelihood.

\section{Results:}

Clinical and EEG data was available for a total 173 subjects. Mean age was 55.3 years and overall mortality was $55.7 \%$. One hundred and twenty subjects $(69.4 \%)$ had poor outcome. Our multiparametric QEEG method achieved optimal performance for mortality prediction at 24 hours (AUC 0.75 ), with a sensitivity of $86 \%$ and specificity of $66 \%$. Optimal poor outcome prediction performance was achieved at 26 hours (AUC 0.72 ), with a sensitivity of $88 \%$ and specificity of $70 \%$. At a false-positive rate of $0 \%$, the sensitivity for poor outcome was $53 \%$. Alpha-delta ratio and voltage $<20 u \mathrm{~V}$ were independently associated with mortality and poor discharge outcome at 24 hours $(p<0.05)$.

\section{Conclusions:}

Employment of machine-learning methods in QEEG analysis allows early and robust outcome prediction in cardiac arrest. This approach has potential to facilitate real-time individualized prognostication in cardiac arrest. 
Poster 126

\title{
Impact of Secondary Brain Injury on Functional Outcome in ECMO-treated Adults after Potentially Reversible Cardio-respiratory Failure
}

\author{
Haroon Majoka, Aashish A. Anand, Imad I. Khan, Zaka Z. Ahmed, Christopher C. Melinosky, Sara S. \\ Hefton, Wendy W. Chang, Melissa M. Motta, Daniel D. Herr, Neeraj N. Badjatia, Paul P. McCarthy, \\ Gunjan G. Parikh.
}

Neurocritical Care, Program in Trauma, Univ. of Maryland School of Medicine, Baltimore, USA.

\section{Introduction:}

Secondary brain injury may be a significant barrier to survival following extracorporeal membrane oxygenation (ECMO) for, otherwise reversible, cardiorespiratory failure. Prevalence of brain injury phenotypes on neuroimaging were described in our prior work. This study aims to study the impact of neurological injury on outcomes in adult patients on ECMO.

\section{Methods:}

A retrospective cohort of ECMO-treated adults. Clinical and outcome data was obtained from electronic chart abstraction of clinical and physical/occupational therapy records.

\section{Results:}

190 patients were studied over 29 months. $37 \%$ underwent neuroimaging during ECMO or within 1 week of decannulation. $27 \%(\mathrm{~N}=18)$ of $\mathrm{CT}$ scans and $93 \%(\mathrm{~N}=13)$ of MRIs had abnormal findings. Intracranial hemorrhage was seen in $20 \%$ patients with neuroimaging. In addition, $86 \%$ of MRIs revealed diffuse microbleeds. $16 \%$ patients had ischemic strokes. $9 \%$ had evidence for global edema. There was no significant difference in survival to hospital discharge and mean modified Rankin scores (mRS) in patients with or without neuroimaging during ECMO (64\% vs.54\%, $\mathrm{p}=0.2$; $\mathrm{mRS}, 4.8 \pm 1.5$ vs. $4.8 \pm 1.3, \mathrm{p}=0.9)$. However, in the group undergoing neuroimaging, normal scans were associated with better survival to hospital discharge $(79 \%$ vs. $21 \% p<0.001)$ and lower $\mathrm{mRS}(4.4 \pm 1.3$ vs. $5.5 \pm 1.0, p=0.002) .12(20.6 \%)$ of survivors who did not get neuroimaging and $3(8 \%)$ of those who got neuroimaging achieved abilty to perform independent $A D L$ at discharge $(p=0.08)$. All 3 patients with ability to perform independent $A D L$ in neuroimaging group had normal scans. 20 (34\%) of survivors who did not get neuroimaging and $8(21 \%)$ of those who got neuroimaging were discharged home $(p=0.1)$. All 8 surviving patients in the neuroimaging group who were discharged home had normal scans.

\section{Conclusions:}

Secondary brain injury in ECMO-treated adults with otherwise reversible cardiac/pulmonary failure affects survival and functional outcomes. A prospective study has been planned to better understand mechanisms mediating this effect. 


\title{
Poster 127
}

\section{Effect of Idarucizumab on Intracranial Hemorrhage in Dabigatran-treated Patients: Initial Results from RE-VERSE AD}

\author{
Thorsten Steiner ${ }^{1}$, Richard R. Bernstein ${ }^{2}$, Charles C. Pollack, Jr. ${ }^{3}$, Jeffrey J. Weitz ${ }^{4}$, Paul P. Reilly ${ }^{5}$, John \\ J. Eikelboom ${ }^{4}$, Menno M. Huisman ${ }^{6}$, Pieter P. Kamphuisen ${ }^{7}$, Jörg J. Kreuzer ${ }^{8}$, Jerrold J. Levy' \\ ${ }^{1}$ Klinikum Frankfurt Höchst, Frankfurt, Germany, ${ }^{2}$ Northwestern University, Chicago, IL, USA, ${ }^{3}$ Thomas \\ Jefferson University, Philadelphia, PA, USA, ${ }^{4}$ McMaster University, Hamilton, Ontario, Canada, ${ }^{5}$ \\ Boehringer Ingelheim, Ridgefield, CT, USA, ${ }^{6}$ Leiden University Medical Center, Leiden, Netherlands, ${ }^{7}$ \\ Tergooi Hospital, Hilversum, Netherlands, ${ }^{8}$ Boehringer Ingelheim Pharma GmbH \& Co. KG, Ingelheim, \\ Germany, ${ }^{9}$ Duke University Medical Center, Durham, NC, USA.
}

\section{Introduction:}

RE-VERSE AD is an ongoing, phase 3, cohort study evaluating the extent to which idarucizumab, a humanized Fab fragment specifically reverses dabigatran's anticoagulation effect in patients with serious bleeding or requiring urgent interventions. Dabigatran is a direct acting oral anticoagulant approved for stroke prevention in non-valvular atrial fibrillation and venous thromboembolism treatment and prevention. In RE-LY, dabigatran 150 and $110 \mathrm{mg}$ BID were associated with significantly lower annualized rates of intracranial hemorrhage $(\mathrm{ICH})$ than warfarin $(0.32 \%, 0.23 \%$ and $0.76 \%$, respectively). Nonetheless, the mortality rate with $\mathrm{ICH}$ in the context of any anticoagulation remains high, probably reflecting the effect of hematoma expansion. Whether idarucizumab improves clinical outcome in dabigatran-treated patients with $\mathrm{ICH}$ is unknown.

\section{Methods:}

This analysis of the first 90 patients enrolled in RE-VERSE AD focuses on patients with ICH. Patients presenting with $\mathrm{ICH}$ were given intravenous idarucizumab $5 \mathrm{~g}$ as two $2.5 \mathrm{~g}$ bolus infusions administered $\leq 15$ minutes apart. The primary endpoint was the maximum reversal of dabigatran's anticoagulation effect, based on central laboratory determination of dilute thrombin time (dTT) or ecarin clotting time (ECT). We compared the clinical outcome of this RE-VERSE AD interim analysis with dabigatran-treated $\mathrm{ICH}$ patients in RE-LY.

\section{Results:}

In this interim analysis, 18 patients with dabigatran-associated ICH were enrolled in RE-VERSE AD. Complete reversal of anticoagulation was observed by dTT and ECT within minutes of idarucizumab administration. Preliminary results from this interim analysis indicate that the mortality rate of ICH patients was $22 \%(4 / 18)$ versus $35 \%$ (13/37) and 41\% (11/27) with dabigatran 150 and $110 \mathrm{mg}$, respectively in RE-LY.

\section{Conclusions:}

Idarucizumab reversed anticoagulation in $\mathrm{ICH}$ patients and appears to improve mortality rates in dabigatran-treated patients with ICH versus historical controls from RE-LY. Results from additional patients in RE-VERSE AD will provide further information on the effects of idarucizumab reversal in patients with $\mathrm{ICH}$. 


\section{Poster 128}

The Effect of Andexanet Alfa on the Pharmacokinetics and Renal Clearance of the Direct Factor Xa Inhibitors

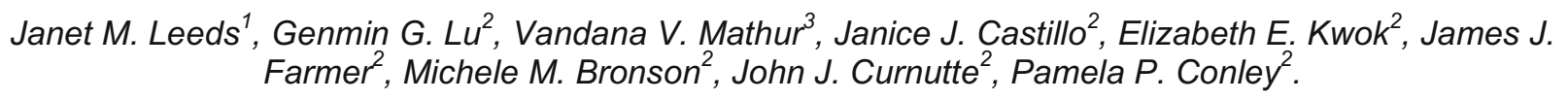

${ }^{1}$ Portola Pharmaceuticals, Inc., South San Francisco, CA, USA, ${ }^{2}$ Portola Pharmaceuticals, Inc., South San Francisco, USA, ${ }^{3}$ Mathur Consulting, Woodside, CA, USA.

${ }^{* * * * \text { Permission was not granted to print this abstract }}{ }^{* * * *}$ 


\title{
Poster 129
}

\section{Sedation Use Alters Quantitative Electroencephalography in Comatose Post-Cardiac Arrest Patients}

\author{
Callie M. Drohan ${ }^{1}$, Alessandra A. Cardi ${ }^{2}$, Jon J. Rittenberger ${ }^{3}$, Clifton C. Callaway ${ }^{3}$, Maria M. Baldwin $^{4}$, \\ Jonathan J. Elmer ${ }^{5}$.
}
${ }^{1}$ University of Pittsburgh School of Medicine University of Pittsburgh Medical Center, Pittsburgh, PA, USA, ${ }^{2}$ University of Pittsburgh School of Medicine, Pittsburgh, PA, USA, ${ }^{3}$ University of Pittsburgh Medical Center, Dept of Emergency Medicine, Pittsburgh, PA, USA, ${ }^{4}$ University of Pittsburgh Medical Center, Dept of Neurology, Pittsburgh, PA, USA, ${ }^{5}$ University of Pittsburgh Medical Center, Dept of Critical Care Medicine, Pittsburgh, PA, USA.

\section{Introduction:}

Electroencephalography (EEG) has clinical and prognostic importance for comatose survivors of cardiac arrest. Recent interest in quantitative EEG (qEEG) analysis has grown. The qualitative effects of sedation on EEG are well known, but potentially confounding effects of sedatives on qEEG are poorly characterized in anoxic injury. We hypothesize that sedation would decrease amplitude-integrated EEG $(\mathrm{aEEG})$ and alpha/delta ratio (ADR), increase suppression ratio (SR), and magnitude of this change would predict neurological recovery.

\section{Methods:}

We routinely monitor comatose post-arrest patients with EEG for 48-72h, or until death or awakening. In this prospective study, we included consecutive EEG-monitored patients who had protocolized sedation interruptions, excluding those with contraindications to interruption such as seizure or hemodynamic instability. We used Persyst v12 to quantify SR, aEEG, and ADR and calculated medians for 10min immediately prior to sedation interruption and the last $5 \mathrm{~min}$ of interruption. We used nonparametric tests to determine if the qEEG signal changed pre- to post- and whether this differed by outcome (Cerebral Performance Category 1-2 at hospital discharge vs 3-5).

\section{Results:}

Of 101 screened subjects, 22 met inclusion criteria (median age 58 years, $73 \%$ male). Sedation regimens varied (18 propofol; 13 fentanyl; 5 midazolam). Median duration of sedation interruption was 35min, and did not differ by sedative type. Pre-interruption, higher ADR and aEEG and lower SR predicted favorable outcome. Post-interruption, SR decreased (median change -0.85 , IQR: -12.0 to 0 ), aEEG increased (0.38, IQR +0.02 to 1.07), but ADR did not change. SR decreased more among those with a poor outcome $(P=0.01)$, but aEEG and ADR changes did not differ by outcome.

\section{Conclusions:}

In acute anoxic brain injury, sedation increases SR and decreases aEEG. Larger decreases in SR with sedation interruption predict worse outcomes, which may reflect a susceptibility of deafferentated cortex to suppress in response to sedation. 
Poster 130

\section{Retrospective Analysis of Levetiracetam Dosing in Neurosurgical Patients for Seizure Prophylaxis}

Ashley R. Hedges, Gary G. Davis, Brianne B. Wolfe, Erin E. Lingenfelter, Gregory G. Hawryluk, Safdar S. Ansari.

University of Utah Hospital and Clinics, Salt Lake City, UT, USA.

\section{Introduction:}

For patients presenting with subarachnoid hemorrhage (SAH) or traumatic brain injury (TBI), levetiracetam has begun to emerge as a preferred alternative to phenytoin for seizure prophylaxis following initial presentation. However, the optimal dose of levetiracetam has not been determined.

\section{Methods:}

A retrospective review of electronic medical records identified patients that received levetiracetam for seizure prophylaxis for SAH or TBI in a level one trauma center from May 12, 2014 to October 30, 2015. The goal of this research was to quantify the combined seizure incidence (including both clinically observed seizures and those confirmed by electroencephalogram) in patients receiving levetiracetam 500 $\mathrm{mg}$ twice daily compared to levetiracetam $>1000 \mathrm{mg}$ total daily dose.

\section{Results:}

Among 139 patients captured, $50 \%$ were male, with a mean age of 53 years old. For patients receiving levetiracetam doses $>1000 \mathrm{mg}$ total daily dose, 77/78 patients received $1000 \mathrm{mg}$ twice daily. A higher seizure incidence was observed, $11.5 \%$ vs $1.3 \%$, in patients receiving levetiracetam $500 \mathrm{mg}$ twice daily compared to levetiracetam doses $>1000 \mathrm{mg}$ total daily dose, respectively $(7 / 61 \mathrm{vs} 1 / 78$ patients, $p=0.01)$. This was observed despite no difference in potential confounders, including history of alcoholism (9/61 vs $15 / 78, p=0.49)$, benzodiazepine use (40/61 vs $57 / 78, p=0.28)$, or propofol use $(38 / 61$ vs $49 / 78, p=0.17)$. A trend towards increased levetiracetam failure rates was observed in the lower dosing scheme of $500 \mathrm{mg}$ twice daily. This was captured by the following: higher rates of levetiracetam dose escalation (12/61 vs $4 / 78, p=0.01)$, addition of other antiepileptic agents (6/61 vs $6 / 78, p=0.25)$, and number of electroencephalograms ordered (22 vs $16, p=0.18$ ). No difference in adverse effects were observed (anemia $1 / 61$ vs $3 / 78, p=0.44$; thrombocytopenia $1 / 61$ vs $1 / 78, p=0.86$ ).

\section{Conclusions:}

Our project suggests that patients may benefit from a standardized levetiracetam dosing scheme of 1000 mg twice daily. 


\title{
Poster 131
}

\section{Fibrinolytic For Treatment Of Intraventricular Hemorrhage: Meta Analysis And Literature Review}

\author{
Alexandra D. Baker ${ }^{1}$, Krissia K. Rivera Perla ${ }^{1}$, Rachel R. Dlugash ${ }^{1}$, Wendy W. Ziai' ${ }^{1}$ Issam I. Awad ${ }^{2}$, \\ Daniel D. Hanley'. \\ ${ }^{1}$ Johns Hopkins Hospital/ Department of Brain Injury Outcomes, Baltimore, MD, USA, ${ }^{2}$ University of \\ Chicago/ Department of Neurology, Chicago, IL, USA.
}

\section{Introduction:}

Treating intraventricular hemorrhage (IVH) with a fibrinolytic (IVF) therapy such alteplase via a catheter is becoming an increasingly popular intervention. With the conclusion of the CLEAR III trial a larger cohort of patient data is available to update past meta analyses. Mortality and good functional outcome after thrombolytic treatment was reviewed in patients with hypertensive IVH.

\section{Methods:}

A literature search was performed from 1970 to March 2016 to collect all literature on IVH treatment with IVF. Seventeen papers meeting our inclusion and exclusion criteria were collected for further analysis. IVH patients with vascular abnormalities and traumatic injuries were excluded. Mortality and functional outcome was assessed to compare IVF treated and control groups in all publications.

\section{Results:}

There was a significant difference in mortality favoring the treatment group at 30 days, 180 days and 365 days $(95 \% \mathrm{Cl}, 0.43-0.75 ; \mathrm{P}=0.000 ; 95 \% \mathrm{Cl}, 0.45-0.82 ; \mathrm{P}=0.001 ; 95 \% \mathrm{Cl}, 0.50-0.90 ; \mathrm{P}=0.008)$. Pooling $\mathrm{mRS}$ and GOS good functional outcomes, there was a significant difference favoring the treatment group at 30,90 , and 180 days $(95 \% \mathrm{Cl}, 0.80-0.96 ; \mathrm{P}=0.003 ; 95 \% \mathrm{Cl}, 0.41-0.68 ; \mathrm{P}=0.000 ; 95 \% \mathrm{Cl}, 0.73-0.96$; $\mathrm{P}=0.012$ ). There was a non-significant trend in $\mathrm{mRS}$ scores favoring the treatment group. A significant difference in GOS score favoring the treatment group was found at $30,90,180$, and 365 days $(95 \% \mathrm{Cl}$, $0.61-0.95 ; \mathrm{P}=0.016 ; 95 \% \mathrm{Cl}, 0.36-0.70 ; \mathrm{P}=0.000 ; 95 \% \mathrm{Cl}, 0.52-0.88 ; \mathrm{P}=0.0003 ; 95 \% \mathrm{Cl}, 0.54-0.95$; $P=0.019)$.

\section{Conclusions:}

Treatment of hypertensive IVH with thrombolytic may improve functional outcome and reduce mortality compared to control groups as early as 30 days, a trend that continues to 365 days for mortality and 180 days for functional outcome. Different effect sizes are generated when different functional outcome tools, such as $\mathrm{mRS}$ and GOS, are used. 
Poster 132

\title{
Warfarin Subdural Hematoma (SDH) Re-Evacuation is More Common than Antiplatelet Associated SDH
}

\author{
Kelly Gassie ${ }^{1}$, William W. Freeman ${ }^{2}$, Kristine K. Thompson ${ }^{3},{ }_{3}$ H. Gordon H. Deen ${ }^{4}$, Robert R. Wharen ${ }^{4}$, \\ Charles C. Graham ${ }^{3}$.
}

${ }^{1}$ Mayo Clinic Florida, Jacksonville, FL, USA, ${ }^{2}$ Mayo Clinic Florida/Neurosurgery/Critical Care Department, Jacksonville, FL, USA, ${ }^{3}$ Mayo Clinic Florida/Emergency Department, Jacksonville, FL, USA, ${ }^{4}$ Mayo Clinic Florida/Neurosurgery, Jacksonville, FL, USA.

\section{Introduction:}

An aging population and increasing use of anticoagulants and antiplatelet agents to prevent ischemic stroke from atherothrombotic disease and atrial fibrillation poses risks of spontaneous subdural hematoma (sSDH) and SDH related to trauma (tSDH). We sought to study the association of antiplatelet agent and warfarin use in SDH patients admitted to our neurosurgical ICU.

\section{Methods:}

All SDH admitted to the neurolCU at Mayo Clinic in Florida who were taking antiplatelet agents and warfarin were studied. Neurosurgical methods of SDH evacuation and re-evacuation were studied as well as hemostatic factors such as international normalized ratio (INR) within the first $24 \mathrm{hrs}$, blood products and hemostatic agents given to reverse coagulopathy or antithrombotic effects. Demographic information such as age, gender and comorbidities were noted and indication for antithrombotic agent. We excluded major trauma (Level 1) associated with SDH at our center.

\section{Results:}

From January 2006 to May 2016, we admitted 188 SDH patients, of which $46.8 \%$ required evacuation (88 surgeries/188 patients), 44 were on warfarin (admission INR range $1.1->12$, mean $=2.9$ ), and 58 on antiplatelet agents. Of those who underwent initial SDH evacuation (24/44) on warfarin, 8/24 (33\%) underwent redo evacuation (range in days 1-24) compared to only $7 / 30(23.3 \%)$ on antiplatelet agents (date range 1-10 days). 24 hour INR rates in Warfarin related SDH were all $<1.5$ except for 6 . Of these 6 , only one patient required re-operation. Mortality in the warfarin group was higher $13.6 \%(6 / 44)$ by discharge or by 30 days compared to $6.9 \%(4 / 58)$ in the antiplatelet arm.

\section{Conclusions:}

In our patient population, SDH was associated with a need for evacuation in $46.8 \%$ of all patients, of which a higher rate of re-evacuation required in those on warfarin compared to those taking antiplatelet agents. Mortality was also higher in the warfarin associated SDH patients. 


\section{Poster 133}

\section{The Ability to Predict Long-term Outcome Differs Between Health Care Provider Teams in Mechanically Ventilated Neurocritical Care Patients} Anna Finley Caulfield, MD, Michael M. Mlynash, MD, MS, Irina I. Eyngorn, MD, Anousheh A. Afjei, MD,
Chitra C. Venkatasubramanian, MBBS, MD, Marion M. Buckwalter, MD, PhD, Karen K. Hirsch, MD.

Stanford University Department of Neurology and Neurological Sciences, Palo Alto, CA, USA.

\section{Introduction:}

Predictions of functional clinical outcome in neurocritical care patients' impact care decisions. This study compared the accuracy of prognostic predictions among health care providers with and without neurological training.

\section{Methods:}

Consecutive patients who were intubated for $\geq 72$ hours with primary neurological illness or neurological complications were prospectively enrolled. Medical critical care attendings (MICU), neurocritical care attendings (NICU), residents (RES), and nurses (RN) predicted the following: 1) 6-month functional outcome measured by modified Rankin scale score (mRS), and 2) 6-month quality of life (QOL). Patients were followed up at 6 months and their functional status and QOL were compared to the predicted values. Functional outcomes were dichotomized to good (mRS 0-3) vs. poor (mRS 4-6).

\section{Results:}

179 (of 365) patients had 6-month mRS predicted by all 4 provider groups. Fifty-four (30\%) patients had good outcome and $125(70 \%)$ had poor outcome. The MICU, NICU, RES, and RN providers had similar predictive values $(95 \% \mathrm{Cl}$ ) for accurately predicting good outcome (65\% (52-76), 62\% (51-72), 70\% (5583 ), and $64 \%$ (50-76), respectively). NICU was most accurate in identifying poor patient outcome, $97 \%$ (91-99), followed by MICU 89\% (74-86), RN 86\% (78-91), and RES 83\% (75-89) ( $p=0.025,0.005$, and $<0.001$, respectively). When patients who transitioned to comfort measures only $(n=65)$ were excluded from the analysis, the NICU team was more accurate at predicting poor outcome. Fifty-three survivors had QOL predicted by all 4 provider groups. The accuracy of QOL predictions for good/excellent QOL and for poor/fair QOL did not differ between provider groups.

\section{Conclusions:}

Neurocritical care attendings are better than healthcare providers without neurological training at predicting poor 6-month functional outcome in neurocritical care patients. However, the overall predictive accuracy for 6-month $\mathrm{mRS}$ and QOL was similar between healthcare provider teams. There are significant limitations in providers' ability to predict long-term functional outcomes. 


\title{
Poster 134
}

\section{"Is There hope? Is She There?" - Perspectives Of Family Members, Nurses, And Physicians Of Patients With Severe Acute Brain Injury}

\author{
Rachael E. Schutz ${ }^{1}$, Claire C. Creutzfeldt ${ }^{2}$, Heather H. Coats ${ }^{3}$, Randall R. Curtis ${ }^{4}$, Ruth R. Engelberg ${ }^{4}$. \\ ${ }^{1}$ Vanderbilt University School of Medicine, Nashville, TN, USA, ${ }^{2}$ University of Washington/Department of \\ Neurology, Seattle, WA, USA, ${ }^{3}$ University of Washington/Cambia Palliative Care Center of Excellence, \\ Seattle, WA, USA, ${ }^{4}$ University of Washington/Cambia Palliative Care Center of Excellence, Seattle, WA, \\ USA.
}

\section{Introduction:}

Patients with severe acute brain injury $(\mathrm{SABI})$ raise important palliative care considerations associated with sudden, devastating injury and uncertain prognosis. The goal of this study was to explore how family members, nurses and physicians experience the palliative and supportive care needs of patients with $\mathrm{SABI}$ receiving care in the neurosciences intensive care unit (neuro-ICU).

\section{Methods:}

Design: Semi-structured in-person interviews were audiotaped, transcribed, and analyzed using thematic analysis. Setting: Thirty-bed neuro-ICU in a regional comprehensive stroke and level 1 trauma center. Subjects: Forty-seven interviews were completed regarding 15 patients receiving care in the neuro-ICU with family members $(n=16)$, nurses $(n=15)$ and physicians $(n=16)$.

\section{Results:}

Two themes were identified: (1) Hope and (2) Personhood. (1) Families linked prognostic uncertainty to a need for hope and expressed a desire for physicians to acknowledge this relationship. The language of hope varied depending on the participant: clinicians used hope as an object that can be given or taken away, generally in the process of conveying prognosis, while families expressed hope as an action that supported coping with their loved one's acute illness and its prognostic uncertainty. (2) Participants described the loss of personhood through brain injury, the need to recognize and treat the brain-injured patient as a person, and the importance of relatedness and connection, including personal support of families by clinicians. In their pursuit to recognize and preserve personhood, physicians used stories from patients and families to inform them about patient identities, while nurses focused on providing supportive, empathetic care to patients and families.

\section{Conclusions:}

Support for hope and preservation of personhood challenge care in the neuro-ICU as identified by families and clinicians of patients with severe acute brain injury. Specific practical approaches can address these challenges and improve care to meet the needs of patients and families in the neuro-ICU. 


\section{Poster 135}

Andexanet Alfa, an Investigational Universal Antidote for Reversal of Anticoagulation of Factor Xa Inhibitors

Genmin Lu', John J. Curnutte ${ }^{2}$, Mark M. Crowther ${ }^{3}$, Janet J. Leeds ${ }^{2}$, Polly P. Pine ${ }^{2}$, Brian B. Wiens ${ }^{2}$, Vandana V. Mathur ${ }^{4}$, Janice J. Castillo ${ }^{2}$, Pamela P. Conley ${ }^{2}$, Alex A. Gold ${ }^{2}$, Stuart S. Connolly.

${ }^{1}$ Portola Pharmaceuticals, Inc., South San Francisco, CA, USA, ${ }^{2}$ Portola Pharmaceuticals, Inc., South San Francisco, USA, ${ }^{3}$ McMaster University and St Joseph's Hospital, Hamilton, Canada, ${ }^{4}$ Mathur Consulting, Woodside, USA, ${ }^{5}$ McMaster University, Hamilton, Canada. 
Poster 136

\title{
Multicenter Observational Study of Early Mobilization in Neurocritical patients: Implementation of a Protocol for Progressive Mobility.
}

\author{
Fernando Goldenberg ${ }^{1}$, Nicolas N. Ciarrocchi ${ }^{2}$, Carlos C. Perez Acosta ${ }^{2}$, Rolando R. Baez ${ }^{2}$, Foda F. \\ Rosciani ${ }^{2}$, Nadia N. Tessore ${ }^{2}$, Mario M. Teran Salazar ${ }^{2}$, Victoria V. Marquevich ${ }^{3}$, Cecilia C. Kaspar ${ }^{3}$, \\ Pablo P. Pratesi ${ }^{3}$, Sebastian S. Cosenza ${ }^{3}$, Eduardo E. San Roman', Osvaldo O. Patiño ${ }^{2}$, Ana A. Diaz', \\ Alejandro A. Midley², Janet J. Bettger 4 .
}

\author{
${ }^{1}$ Neurocritical Care, Hospital Italiano de Buenos Aires and University of Chicago, Buenos Aires, \\ Argentina, ${ }^{2}$ Hospital Italiano de Buenos Aires, Critical Care Medicine, Buenos Aires, Argentina, ${ }^{3}$ Hospital \\ Universitario Austral, Critical Care Medicine, Buenos Aires, Argentina, ${ }^{4}$ Duke University, Duke Global \\ Health Institute, Durham, NC, USA.
}

\section{Introduction:}

Despite increasing evidence that early mobilization strategies are effective, we showed patients were not adequately mobilized in two Argentinean hospitals. We implemented a progressive-mobility protocol and examined its feasibility, safety and applicability in our neurocritical patients.

\section{Methods:}

Prospective observational implementation study of a progressive-mobility protocol for neurocritical patients admitted to ICUs of two university hospitals in Argentina. All patients were evaluated twice daily for level of movement and clinical stability. Patients progressed as tolerated from passive movement (level 1) to ambulation (level 5). This pre/post analysis compared performance of a two month preimplementation baseline to ICU patients admitted in months 3-4 after implementation began (two month start-up phase not analyzed).

\section{Results:}

There were 33 pre-implementation patients with 348 assessments (40\% post-operative, $28 \%$ traumatic brain injury, $12 \%$ stroke) compared with 60 post-implementation patients with 444 measurements $(55 \%$ post-operative, $7 \%$ traumatic brain injury, $35 \%$ stroke). Baseline demographics were similar between populations: median age 53 years $(95 \% \mathrm{Cl} 44-57)$, nearly $60 \%$ were men. Mobilization was 16.4 times more likely $(95 \% \mathrm{Cl}$ 9.8-27.4) after protocol implementation. Two thirds of pre-implementation patients $(65.2 \%)$ were not mobilized compared with only $9.2 \%$ post-implementation $(p<0.001)$. Among mechanically ventilated patients, $82.2 \%$ of pre-implementation assessments showed no mobilization vs. $8.6 \%$ post. Post-implementation patients with an endotracheal tube had a lower rate of mobilization $(78.9 \%)$ than ventilated patients with a tracheostomy (94.3\%). Passive movement, turns and full assistance to sit up and transition out of bed to chair was achieved for $64.2 \%$ and $26.6 \%$ achieved higher levels $(3,4,5)$. Mobility sessions with the physical therapist were $<20$ minutes in $90 \%$ of the cases. No mobility-related adverse events occurred.

\section{Conclusions:}

Patients were sixteen times more likely to be mobilized following protocol implementation. This prospective study demonstrated that early and progressive mobility among neurocritical care patients in Argentina is feasible and safe. 


\title{
Poster 137
}

\section{Critical IIIness Resilience and Spirituality: Unique Methodology For Surrogate Validation of CD- RISC-10 and Brief RCOPE Spirituality Scales}

\author{
Neha S. Dangayach ${ }^{1}$, Steohen S. Griffiths ${ }^{2}$, Anil A. Ramineni ${ }^{1}$, Maggie M. Keough ${ }^{3}$, Vansh V. Sharma ${ }^{3}$, \\ Danielle D. Wheelwright ${ }^{2}$, Deborah D. Marin ${ }^{3}$, Umesh U. Gidwani, Joshua J. Bederson ${ }^{5}$, J. Mocco ${ }^{5}$, \\ Errol E. Gordon ${ }^{1}$, Stephan S. Mayer'.
}

\begin{abstract}
${ }^{1}$ Mount Sinai Health System/Neurosurgery and Neurology, New York, NY, USA, ${ }^{2}$ Mount Sinai Health System/Institute of Critical Care Medicine, New York, NY, USA, ${ }^{3}$ Mount Sinai Health System/Spiritual Care Department, New York, NY, USA, ${ }^{4}$ Mount Sinai Health System/Mount Sinai Heart, New York, NY, USA, ${ }^{5}$ Mount Sinai Health System/Neurosurgery, New York, NY, USA.
\end{abstract}

\section{Introduction:}

Psychiatry, geriatrics, and oncology have adopted comprehensive approaches to predict outcomes accounting for important constructs such as spirituality and resilience. Critical illness often occurs as a sudden catastrophic event leaving patients with significant long-term cognitive, behavioral and neurological disturbances. Impact of resilience and spirituality on recovery in this setting has not been investigated. We have designed a study to validate two important scales, Connor Davidson Resilience Scale-10 and Brief RCOPE Spirituality scales for surrogate responders.

\section{Methods:}

English or Spanish speaking adults admitted to the Neuro-ICU with an expected length of stay of $\geq 48$ hours with one or two surrogate responders will be included. This prospective cohort study will collect demographic, laboratory and radiographic data in a RedCap database. For every patient enrolled, the CD-RISC-10 and Brief RCOPE Scale will be administered to two next of kin - one immediate next of kin and another next of kin who knows the patient well. Both will be asked to complete scales on patient's behalf, themselves, and for each other. Each patient will complete two resilience and two spirituality scale forms while each next of kin will complete three CD-RISC and three Spirituality scales. The scales will be administered to the patient, if possible, prior to discharge; at 3 months and at 12 months. If patient remains incapacitated, these forms will be administered to immediate next of kin/surrogate during followup.

\section{Results:}

CARS study has screened 44 patients in 35 days, enrolling 30 patients. Common diagnoses include subarachnoid hemorrhage (8), CNS malignancy (5), intracranial hemorrhage (3), unruptured aneurysm (3), subdural hematoma (2).

\section{Conclusions:}

Based on current enrollment, this unique methodology for surrogate validation of scales is feasible. By August 2016, an anticipated 150 subject will be recruited. Surrogate validation of quantitative measurements of resilience, spirituality can provide new insight into prognostication and patient centered critical care. 
Poster 138

\title{
Increasing Family Engagement in Neurocritical Care: A Qualitative Study of Perceptions and Attitudes Regarding Passive Mobilization
}

\author{
Fernando D. Goldenberg ${ }^{1}$, Foda F. Rosciani ${ }^{2}$, Rolando R. Baez ${ }^{2}$, Nicolas N. Ciarrocchi', Nadia N. \\ Tessore $^{2}$, Carlos C. Perez Acosta ${ }^{2}$, Catalina C. Jerez Fonseca ${ }^{3}$, Nahuel N. Braguinsky ${ }^{3}$, Eduardo E. San \\ Roman ${ }^{4}$, Janet J. Bettger ${ }^{5}$, Adriana A. Dawidowski .
}

\begin{abstract}
${ }^{1}$ Neurocritical Care. Hospital Italiano - Buenos Aires and University of Chicago, Buenos Aires, Argentina, ${ }^{2}$ Neurocritical Care. Hospital Italiano de Buenos Aires, Buenos Aires, Argentina, ${ }^{3}$ Epidemiology Section. Hospital Italiano de Buenos Aires, Buenos Aires, Argentina, ${ }^{4}$ Care Care Medicine. Hospital Italiano de Buenos Aires, Buenos Aires, Argentina, ${ }^{5}$ Duke University. Duke Global Health Institute, Durham, NC, USA.
\end{abstract}

\section{Introduction:}

Active family engagement in the intensive care unit (ICU) could improve patients' and families' experience with care, interactions with the healthcare team, and outcomes. This study examined the perceptions and attitudes of family members regarding increased engagement with passive mobilization of neurocritical care patients.

\section{Methods:}

An educational video on passive mobilization of ICU patients was developed to engage family members to participate as valued members of the healthcare team. An anthropologist and a nurse or physician (study team) invited family of neurocritical care patients in an academic medical center ICU in Argentina to watch the video. The study team observed reactions to the video and asked families to reflect on the content and describe their attitudes toward involvement. One week later, a second interview explored their perceptions of engagement with care. A multidisciplinary team (3 MDs, 2 RNs, 2 anthropologists) completed the analysis.

\section{Results:}

Thirty-two family members ( $53 \%$ female) of 16 ICU patients participated. The study team observed and the family participants reported to be positively surprised by: the format of learning by video that was different from how they usually received daily information; the information in the video was more detailed than anything previously taught; the invitation to watch the video and engage in patient's care was new and unexpected as was the opportunity to provide opinions on how to improve the video instruction. A week later they perceived the instruction on passive mobilization as an experience that demystified and newly authorized them to touch the patient and participate in care. They reported increased hope about prognosis and perceived an improved relationship with the healthcare team. Only one family member considered the video irrelevant.

\section{Conclusions:}

Family members' attitudes and perceptions toward this low cost approach to engagement were positive. This approach to teaching and engagement may help humanize the complex ICU environment. 


\title{
Poster 139
}

\section{"I believe in miracles": Implications for the Neuro-ICU}

\author{
Anil Ramineni ${ }^{1}$, Neha N. Dangayach ${ }^{2}$, Stephen S. Griffiths ${ }^{3}$, Maggie M. Keogh ${ }^{3}$, Danielle D. Wheelwright ${ }^{3}$, \\ Vansh V. Sharma ${ }^{3}$, Stephan S. Mayer ${ }^{3}$, Umesh U. Gidwani, Javaad J. Ahmad ${ }^{3}$, Joshua J. Bederson ${ }^{3}, J^{3}$ \\ J. Mocco $^{3}$. \\ ${ }^{1}$ Mount Sinai Hospital / Neurosurgery \& Critical Care, New York, NY, USA, ${ }^{2}$ Mount Sinai Hospital, New \\ York, NY, USA, ${ }^{3}$ Mount Sinai Hospital, New York, USA.
}

\section{Introduction:}

$70-80 \%$ of Americans believe in the concept of miracles. We hypothesize that a belief in miracles leads to a higher rate of tracheostomies/PEG tubes, longer hospital length of stay, full code status, and lower consultation rate of palliative care. Addressing a patient or family's belief in miracles and understanding what a miracle signifies early in the Neuro-ICU may assist in important shared decision making.

\section{Methods:}

The Critical Illness Resilience and Spirituality (CARS) study is a prospective cohort study currently recruiting English or Spanish speaking adults admitted to the Neuro-ICU at Mount Sinai Hospital with an expected length of stay of at least 48 hours and surrogate responders. As part of the study, all recruited patients and their families are asked about their belief in miracles on admission. Their responses are stratified into two categories: belief in miracles, or an uncertainty/disbelief with regards to miracles. Our primary outcome is tracheostomy and PEG tube placement. Secondary outcomes include length of stay, full code status, and palliative care consultation. These groups will be matched with regards to the underlying illness; for example, acute ischemic stroke. Severity of illnesses measured by SOFA score and disease specific severity score; for example, NIH Stroke Scale. Statistical analysis utilizing logistic regression will be used to compare rates of the primary and secondary outcomes.

\section{Results:}

The CARS study has screened 44 patients screened and enrolled 30 patients so far. Of those enrolled, the most common diagnoses include subarachnoid hemorrhage (8), CNS malignancy (5), intracranial hemorrhage (3), unruptured aneurysm (3), subdural hematoma (2).

\section{Conclusions:}

This is an ongoing study, we anticipate recruiting 150 patients by the end of August, 2016. Belief in miracles could potentially influence continuation of aggressive measures in a shared decision-making paradigm in the Neuro-ICU. 
Poster 140

\title{
Neurological Prognostication and Withdrawal of Life-Sustaining Therapy (WLST) practices for Post-Cardiac Arrest Patients at a Single Academic Medical Center: A Retrospective Analysis
}

\author{
Mary M. Barden, Teddy T. Youn, Carolina C. Maciel, Sonya S. Zhou, David D. Greer.
}

Department of Neurology, Yale-New Haven Hospital, Yale School of Medicine, New Haven, CT, USA.

\section{Introduction:}

Withdrawal of life-sustaining therapy (WLST) for predicted poor neurological outcome is a common cause of death among post-cardiac arrest patients. Recent guidelines recommend against WLST before 72 hours post-arrest. Early WLST perpetuates a self-fulfilling prophecy that may contribute to premature death in some patients who otherwise would have survived with good neurological recovery.

\section{Methods:}

A retrospective cohort of resuscitated cardiac arrest patients from January 2011 to March 2016 at a single tertiary academic medical center was reviewed. Patients were evaluated for outcomes at hospital discharge and (when applicable) the timing of and reason for WLST. Prognostic indicators including clinical examination, electrophysiology, and neuroimaging were analyzed and findings were compared to day of WLST.

\section{Results:}

Of 144 patients, 64 (44\%) had WLST due to perceived poor neurological prognosis. Median day of WLST for this reason was post-arrest day 6 . When stratified according to treatment with targeted temperature management (TTM), the median day of WLST remained day 6 for both TTM-treated and non TTM-treated groups. Of patients with WLST, the phrase "no chance for meaningful recovery" was used in documentation for $23(36 \%)$, MRI results were cited as indicative of poor neurological prognosis for 16 $(25 \%)$, and pupillary light reflex was present day 3 post-arrest (or day 3 post-complete rewarming) in 35 $(55 \%)$.

\section{Conclusions:}

In a retrospective cohort of resuscitated cardiac arrest patients, WLST for predicted poor neurological outcome was the most common cause of death. The median day of WLST was post-arrest day 6. Many patients with WLST had present pupillary reflexes on day 3 post-arrest (or day 3 post-complete rewarming) and MRI was used as a prognostic tool despite lack of evidence that it is useful as such. Early WLST in the setting of indeterminate prognostic indicators undermines accurate neurological prognostication of post-cardiac arrest patients and perpetuates a self-fulfilling prophecy of poor outcome. 


\title{
Poster 141
}

\section{Invasive Multi-Modality Monitoring of Cerebral Physiology to Guide Therapy in Hypoxic Ischemic Brain Injury}

\author{
Swarna Rajagopalan', Wesley W. Baker', Bethany B. Young ${ }^{1}$, Andrew A. Kofke ${ }^{2}$, Ramani R. Balu1 . \\ ${ }^{1}$ University of Pennsylvania, Neurocritical Care, Philadelphia, PA, USA, ${ }^{2}$ University of Pennsylvania, \\ Anesthesia, Neurocritical Care, Philadelphia, PA, USA.
}

\section{Introduction:}

Global Hypoxic Ischemic Brain Injury (HIBI) is a major cause of death and disability worldwide. Invasive monitoring of brain function enables goal-directed treatment strategies that optimize cerebral physiology, reduce secondary brain injury (SBI), and potentially improve outcomes. We report a series of patients with $\mathrm{HIBI}$ where intracranial monitors were placed to guide clinical management.

\section{Methods:}

Retrospective analysis of 7 patients with HIBI cared for at a large academic center over a 3 year period. All patients received therapeutic hypothermia (TH) to $33^{\circ}$, continuous EEG monitoring, and had a bundle of invasive monitors placed through a multi-lumen cranial bolt. The full bundle consisted of an ICP monitor, brain oxygen (PbtO2) monitor, cerebral blood flow (CBF) probe, and cerebral microdialysis probe. 4 patients received the full bundle, while the others received a partial bundle. Patients were treated using a tiered algorithm designed to optimize cerebral physiological parameters.

\section{Results:}

Precipitants of HIBI included cardiac arrest (5 patients), airway occlusion during anesthesia induction (1 patient), and hanging (1 patient). Mean patient age was 45 years. Average time between initial injury and probe placement was 37 hours. Average duration of monitoring was 6.7 days. No adverse events occurred after monitor placement. Episodes of deranged cerebral physiology-including intracranial hypertension, brain hypoxia, cerebral glycopenia, metabolic crisis, and reduced perfusion leading to treatment changes occurred in 6 of 7 patients. They occurred up to 10 days after initial injury, and in all cases would have otherwise been clinically silent. 4 of 7 patients died in the hospital. The surviving patients all regained consciousness and were discharged to Acute Rehabilitation facilities. We did not find a consistent correlation between markers of cerebral physiology and outcome.

\section{Conclusions:}

Invasive intracranial monitoring after $\mathrm{HIBI}$ appears safe and identifies physiological states associated with $\mathrm{SBI}$. Goal directed treatment utilizing multi-modality monitoring in HIBI merit further study. 


\title{
Poster 142
}

\section{Implementation of a Validated Delirium Prediction Score in Patients with Acute Cerebrovascular Disease}

\author{
Joseph B. Haymore ${ }^{1}$, Corey C. Lewis ${ }^{1}$, Andy A. Shirley ${ }^{1}$, Rebecca R. Horrell ${ }^{1}$, Rachel R. Hausladen ${ }^{1}$, \\ Imad I. Khan ${ }^{2}$, Christopher C. Melinosky', Wan-Tsu W. Chang ${ }^{3}$, Melissa M. Motta ${ }^{2}$, Gunjan G. Parikh', \\ Neeraj N. Badjatia'.
}

\begin{abstract}
${ }^{1}$ Neurocritical Care Advanced Practice Provider Group, Univ. of Maryland Medical Center, Baltimore, MD, USA, ${ }^{2}$ Neurocritical Care Section, Program in Trauma, Univ. of Maryland School of Medicine, Baltimore, MD, USA, ${ }^{3}$ Neurocritical Care Sect, Prog in Trauma and Dept Emer Med, Univ of Maryland School of Med, Baltimore, MD, USA.
\end{abstract}

\section{Introduction:}

Delirium is common in patients with acute ischemic stroke (AIS) and intracerebral hemorrhage (ICH) and is associated with worse patient outcomes; however, it can be difficult to reliably detect. Delirium prevention is therefore a potentially beneficial strategy and is most effective in patients who are at high risk for delirium. We implemented a validated delirium prediction score (DPS) in patients with AIS and ICH to evaluate whether the Advanced Practice Providers (APP's) would both use the DPS and also find the DPS easy to use.

\section{Methods:}

During a 6-week process improvement (PI) pilot, the APPs in a 10-bed Neurocritical Care Unit at a large urban academic medical center used a validated DPS to risk-stratify consecutive admissions of patients with AIS and ICH into low (20\%) risk for delirium. Compliance data was collected and analyzed with descriptive statistics. The APPs completed a 14-item questionnaire that included the System Usability Scale (SUS) and open-ended questions to determine the usability of the DPS, as well as to assess for facilitators and barriers for the use of the DPS. No individual patient data was collected.

\section{Results:}

Patients admitted with AIS and ICH $(n=20)$ were assessed by the APP's using the DPS. Compliance with DPS use was $90 \%(n=18 / 20)$. Two patients were not assessed due to resistance to change by a minority of APPs $(n=2)$. The SUS score (76.7) was mid-point between "acceptable" and "excellent." Facilitators and barriers for use of the DPS were identified.

\section{Conclusions:}

The DPS was easy to use and was consistently used by the APP's. Adoption of the DPS with this patient population can be a first step to identify the most at-risk patients to target delirium prevention strategies in this vulnerable population. 


\title{
Poster 143
}

\section{Neurocritical care Education dUring Residency: OpinioNs (NEURON) Study}

\author{
David P. Lerner ${ }^{1}$, Jennifer J. Kim², Saef S. Izzy².
}

${ }^{1}$ Mass Gen Hospital, Brigham \& Womens Hospital, Harvard Med School Dept of Neurology, Boston, MA , USA, ${ }^{2}$ Mass Gen Hospital, Brigham \& Womens Hospital, Harvard Med School Dept of Neurology, Boston, MA, USA.

\section{Introduction:}

The intensive care unit is a complex learning environment with variability in a number of external factors. Prior studies of neurology residency training in the neurological intensive care unit have focused on general exposure. This study aims to evaluate resident perception of neurocritical care training.

\section{Methods:}

An online survey was sent to program directors and neurocritical care members for distribution to neurology residents. The survey consisted of 20 free-text or selection style questions that focus on resident perception of neurocritical training. Statical analysis for group differences was completed with $\mathrm{t}$ or Fisher exact tests

\section{Results:}

A total of 95 responses ( $4.7 \%$ response rate) was obtained. Of those responders, 54 completed a freetext question regarding needed improvements to neurointensive care training. $53 \%$ responded with needs for educational changes, and these responders did not differ from other responder in average required rotation time, 8.8 and 8.9 weeks respectively $(p=0.58)$, exposure to fellows $(71 \%$ vs $81 \% p=0.38)$, nurse practitioners $(61 \%$ vs $81 \% p=0.15)$, and neurocritical care attendings $(97 \%$ vs $93 \% p=0.59)$.

\section{Conclusions:}

This is the first study to examine neurology residents' concerns with neurocritical care rotations. There is little neurocritical care educational materials focused to neurology residents, but the emergency neurology life support training can provide a framework to construct a curriculum. Further studies to assess the neurocritical care educational training priorities during neurology residency are warranted. 
Poster 144

Institutionally Standardized Education Module \& EMR documentation for Death by Neurological Criteria Evaluation can improve Provider Compliance with Guidelines in a Tertiary Care Medical Center

\author{
Aarti Sarwal', Janet J. Crumpler ${ }^{2}$, Kimberly K. McDonough ${ }^{2}$, Kristi K. Tucker ${ }^{2}$, David D. Bowton ${ }^{2}$, Sudhir \\ S. Datar ${ }^{2}$, Allison A. Brashear ${ }^{2}$. \\ ${ }^{1}$ Wake Forest School of Medicine, Winston Salem, NC, USA, ${ }^{2}$ Wake Forest Baptist Medical Center, \\ Winston Salem, NC, USA.
}

\title{
Introduction:
}

Our institute had several cases of conflict come to light in the evaluation of patients being evaluated for death by neurological criteria. Provider understanding and awareness of clinical guidelines was found to be low across all sub-specialties. It was deemed important to follow appropriate procedures based on published guidelines and a standardized process to provide appropriate care for each patient, optimize ICU resource utilization and strengthen provider and public trust. Due to medical, legal and ethical issues involved, an institutional standard was called for .

\section{Methods:}

After seeking feedback from clinical providers from all sub-specialties about discrepancies, we revised the institutional policy to reflect emphasis of educational gaps and reflect the latest published guidelines and practice updates. We created an education module, a standardized template in electronic medical record incorporating a practical checklist, an order set, an intranet webpage and a tiered consult system to allow escalation in case of conflicts .

\section{Results:}

The project led to increased participation and satisfaction amongst the clinical providers in the ICUs when a patient was clinically suspected to a brain dead. The education toolkit empowered providers with an education source to evaluate patients with a consistent approach based on published practiced parameters. We observed a trend in decrease in length of stay and variance for brain dead patients since the toolkit was implemented in early 2013. There is a general sense/feedback of positive impact on organ donation referrals as well timely initiation and effectiveness of family discussions in irreversibly neurologically impaired patients amongst providers. We are currently working on finding markers of confirming this is in a survey model.

\section{Conclusions:}

A standard care pathway towards evaluation of patients with death by neurological criteria can be successfully implemented at an institutional level in a tertiary care academic medical center. 


\section{Poster 145}

\section{Evaluation of a Novel Noninvasive Cerebral Perfusion Monitor (C-Flow) After Cardiac Arrest}

Charles L. Francoeur ${ }^{1}$, Stephen S. Griffiths ${ }^{2}$, Danielle D. Wheelwright ${ }^{2}$, Daniel D. Singer ${ }^{3}$, Neha N. Dangayach ${ }^{2}$, Errol E. Gordon ${ }^{2}$, Anil A. Ramineni , Javaad J. Ahmad', Umesh U. Gidwani ${ }^{4}$, Stephan S. Mayer ${ }^{2}$.

\footnotetext{
${ }^{1} \mathrm{CHU}$ de Québec - Université Laval / Department of Anesthesiology and Critical Care, Québec, Canada, ${ }^{2}$ Icahn School of Medicine at Mount Sinai / Department of Neurology, New York, NY, USA, ${ }^{3}$ Icahn School of Medicine / Department of Emergency Medicine, New York, NY, USA, ${ }^{4}$ Icahn School of Medicine / Department of neurology, New York, NY, USA, ${ }^{5}$ Icahn School of Medicine at Mount Sinai / Cardiovascular Intensive Care Unit, New York, NY, USA.
}

\section{Introduction:}

Establishing and maintaining optimal brain perfusion is a crucial endpoint for resuscitation and postcardiac arrest care. A recently FDA-approved device that employs laser and pulsed Doppler now provides clinicians with the Cerebral Flow Index (CFI), a non-invasive measure of brain perfusion. We sought to determine if CFI provided by the ORNIM C-Flow device can be used as a simple and valid measurement of brain perfusion after resuscitation in cardiac arrest patients.

\section{Methods:}

We performed a single-center prospective observational inception cohort study of adult patients with cardiac arrest starting in October 2015. Comatose patients with sustained return of spontaneous circulation (ROSC) within 60 minutes of maneuvers were included. The ORNIM CFlow was connected as soon as feasible after ROSC. Clinicians were blinded to CFI values. Primary outcome was survival at discharge and secondary outcome was neurological assessment using the Cerebral Performance Categories (CPC) scale at discharge.

\section{Results:}

A total of 24 patients have been enrolled as of May 2016. Half (46.1\%) were out-of-hospital arrests and half $(50 \%)$ had FV/TV as the initial rhythm. The overall survival at discharge was $50 \%$, and $25 \%$ had good neurological outcome (CPC 1 or 2). Mean interval between arrest and start of monitoring was 15 hours with a mean duration of 47 hours. Adequate signal was available $83.4 \%$ of the monitoring time. Mean CFI in survivors was 62.3, compared to 54.6 in non-survivors ( $P$ value 0.07 ). Patients with good neurological outcome at discharge also had a higher mean CFI, although the small sample size precludes any conclusion.

\section{Conclusions:}

Our results demonstrate that cerebral perfusion monitoring using the ORNIM CFlow after cardiac arrest is feasible. It also suggests that higher CFI might be associated with survival at discharge. As enrollment progresses and more data are collected, further insight on the potential role of CFI as a neuromonitoring tool might emerge. 
Poster 146

Neurological Complications after Tuberculous Meningitis in the United States

\author{
Alexander E. Merkler ${ }^{1}$, Alexandra A. Reynolds ${ }^{2}$, Gino G. Gialdini ${ }^{3}$, Nicholas N. Morris ${ }^{2}$, Santosh S. \\ Murthy ${ }^{3}$, Kiran K. Thakur ${ }^{2}$, Hooman H. Kamel ${ }^{3}$. \\ ${ }^{1}$ Weill Cornell Medicine / Neurology, Neurology, NY, USA, ${ }^{2}$ Columbia University / Neurology, New York, \\ NY, USA, ${ }^{3}$ Weill Cornell Medicine / Neurology, New York, NY, USA.
}

\title{
Introduction:
}

Tuberculous meningitis (TBM) is the most devastating form of tuberculosis, yet rates of neurological complications and mortality are uncertain in high-income countries.

\section{Methods:}

We used administrative claims data on all admissions at nonfederal hospitals to identify adult patients with TBM in California between 2005-2011, New York between 2006-2013, and Florida between 20052013. Our outcomes of interest were mortality and the following neurological complications: stroke, seizure, hydrocephalus requiring a ventriculoperitoneal shunt, vision impairment, and hearing impairment. Kaplan-Meier survival statistics were used to assess the cumulative rates of neurological complications and death.

\section{Results:}

We identified 806 patients with TBM, of whom $54.8 \%(95 \% \mathrm{Cl}, 50.9-58.7 \%)$ developed at least one neurological complication or died. More than two-thirds of these complications occurred during the initial hospitalization for TBM. Individual neurological complications were not uncommon: the cumulative rate of stroke was $13.9 \%(95 \% \mathrm{Cl}, 11.3-16.9 \%)$, the rate of seizure was $18.8 \%(95 \% \mathrm{Cl}, 15.4-22.8 \%)$, and the rate of ventriculoperitoneal shunting was $8.4 \%(95 \% \mathrm{Cl}, 6.4-10.9 \%)$. Vision impairment occurred in $21.6 \%$ $(95 \% \mathrm{Cl}, 18.5-25.1 \%)$ of patients and hearing impairment occurred in $6.8 \%(95 \% \mathrm{Cl}, 4.9-9.4 \%)$. The mortality rate was $21.5 \%(95 \% \mathrm{Cl}, 18.4-24.9 \%)$.

\section{Conclusions:}

TBM is associated with a significant risk of neurological complications and death even in high-income countries such as the United States. 


\title{
Poster 147
}

\section{Neuropalliative Care in Peru: Emergence from the Conspiracy of Silence}

\author{
Anastasia Vishnevetsky ${ }^{1}$, Carla C. Zapata del Mar ${ }^{2}$, Juan J. Luis Cam³, Claire C. Creutzfeldt ${ }^{4}$.
}

${ }^{1}$ NPGH Fogarty Scholar, Perelman School of Medicine at the University of Pennsylvania, Philadelphia, PA, USA, ${ }^{2}$ Instituto Nacional de Enfermedades Neoplasticas, Palliative Medicine Service, Lima, Peru, ${ }^{3}$ Instituto Nacional de Ciencias Neurologicas, Intensive Care Unit, Lima, Peru, ${ }^{4}$ University of Washington Harborview Medical Center, Department of Neurology, Seattle, WA, USA.

\section{Introduction:}

The development of palliative care in Peru remains limited, particularly for non-oncologic services such as neurology and neurocritical care. The goal of this study was to explore attitudes and knowledge around palliative and end-of-life care in patients, families, nurses and physicians in a specialized neurological institute in Lima, Peru.

\section{Methods:}

We used a mixed methods approach consisting of 70 surveys and 21 qualitative, semi-structured interviews that were recorded, transcribed and analyzed using thematic analysis.

\section{Results:}

Surveys identified a substantial need for palliative care in the neurological institute (63\% of doctors and $77 \%$ of nurses reported palliative care needs in $>30 \%$ of their patients), as well as a lack of training $(82 \%$ of doctors and $69 \%$ of nurses reported a lack of adequate training in palliative care). The key themes that emerged from qualitative interviews evolved around communication about end-of-life choices in neurologic disease. Knowledge about advance directives was limited among both clinicians and families, and participants were divided about whether or not a patient should know about their serious diagnosis and prognosis, and who should tell them. However, the perception that a physician should be honest, and that suffering and pain should be avoided at all times was unanimous. Barriers to transparency in patientphysician communication included (1) expectation of cure with medical treatment; (2) families' trust in God to determine one's fate; (3) physician's lack of training in communication, symptom management and end-of-life care; and (4) a paternalistic culture. Participants identified several challenges specific to palliative care in neurologic disease.

\section{Conclusions:}

In a country without a palliative care training program and no legal basis for advance directives, families and clinicians are emerging from a culture of silence about serious diagnoses and end-of-life care choices. Our findings emphasize the need for palliative care education for neurology providers and the public in Peru. 
Poster 148

\section{Medical Training and the Brain Death Exam: A Single Institution's Experience}

Kiruba Dharaneeswaran ${ }^{1}$, Ahmed A. Kashkoush ${ }^{2}$, Amy A. Weisgerber $^{3}$, Nitin N. Agarwal', Lori L. Shutter ${ }^{4}$.

${ }^{1}$ Department of Neurology, University of Pittsburgh Medical Center, Pittsburgh, PA, USA, ${ }^{2}$ Department of Neurological Surgery, University of Pittsburgh Medical Center, Pittsburgh, PA, USA, ${ }^{3}$ Center for Organ Recovery \& Education, UPMC, Pittsburgh, PA, USA, ${ }^{4}$ Department of Critical Care Medicine, University of Pittsburgh Medical Center, Pittsburgh, PA, USA.

\section{Introduction:}

A trained physician must perform the brain death examination in a systematic fashion in order to recognize and prevent potential sources of error. Given the infrequency at which brain death presents in a hospital setting, clinicians may not always have the opportunity to observe a brain death examination during their training. In this study, we plan to evaluate the effect of medical specialty and expertise on documentation errors.

\section{Methods:}

We performed a retrospective chart review of 118 brain death examinations between Jan. 12014 to July 31st 2015 at the University of Pittsburgh Medical Center Presbyterian. Physician specialty and training level, documentation errors, and confirmatory tests such as cerebral blood flow (CBF), electroencephalography (EEG), and computed tomography angiography (CTA) were collected from medical records.

\section{Results:}

Critical care medicine attending physicians and fellows carried out 88\% (104/118) of brain death exams. Neurosurgery residents performed $5 \%(6 / 118)$ of the exams and Neurology residents performed $8 \%$ (10/118). Ancillary testing was requested in $10 \%$ of the exams ( 7 CBFs, 4 EEGs, and 1 CTA). Documentation errors occurred in 16\% (19/118) of brain death exams carried out. The most common errors were: lack of documentation of time of death (TOD), incomplete documentation, and incorrect completion of documentation by at least one examiner. Attending physicians, residents and fellows were responsible for $47 \%(9 / 19), 21 \%(4 / 19)$, and $21 \%(4 / 19)$ of the documentation errors respectively.

\section{Conclusions:}

Neurology and Neurosurgery residents have limited exposure to the brain death examination. Regardless of the training level, physicians are susceptible to making documentation errors. 


\title{
Poster 149
}

\section{Pilot Study to Assess Quality of Communication in Acute Neurological Emergencies and Define Domains for Improvement}

\author{
Aarti Sarwal' ${ }^{1}$ Kristopher K. Dixon ${ }^{1}$, Mollie M. Canzona ${ }^{2}$, Michael M. Hyde ${ }^{2}$, Amy A. Guzik ${ }^{1}$, Stacey S. \\ Wolfe ${ }^{1}$, John J. Wilson ${ }^{1}$, Cheryl C. Bushnell'. \\ ${ }^{1}$ Wake Forest School of Medicine, Winston Salem, NC, USA, ${ }^{2}$ Wake Forest University, Department of \\ Communication, Winston Salem, NC, USA.
}

\section{Introduction:}

Communication with patients and their families is of central concern in healthcare. However, evidence shows that it is often poorly addressed, especially at times of rapid health status changes and periods of clinical uncertainty. Acute neurological emergencies pose an inherently unique challenge in communication. While emerging studies have addressed communication gaps and strategies to improve them in various critical care settings, none have assessed this issue in acute neurological emergencies

\section{Methods:}

Ongoing IRB approved prospective observational study in a 24 bed Neurocritical care unit in tertiary care academic medical center All patients admitted to the unit and all clinical providers participating in their care screened for inclusion. Direct observation of discussions between clinical providers and families by a neutral observer. After the encounter, all participants complete a survey with Likert scale and open ended questions addressing the satisfaction, understanding of treatment options, impact on health care decisions and ways to improve communication

\section{Results:}

Five patients have been enrolled in the study, so far. The results are analyzed for concordance between responses of the various participants. A difference of $\geq 2$ or more points between answers is considered disagreement, while a difference of $\leq 1$ is labeled as agreement. Preliminary results show: Agreement between all participants for general satisfaction with the communication (as well as family's understanding of treatment options explained to them by the physician. Some disagreement on the impact of the discussion on health care decisions. Qualitative domains identified by families as areas of good communication included opportunities to ask questions, demeanor and sharing of clinical radiographic images. Domains identified as needing improvement included explanation of medical circumstances and need for private room for discussions.

\section{Conclusions:}

We observed general satisfaction with communication. Further enrollment will help elucidate any definitive areas of improvement and impact of communication on health care decisions. 
Poster 150

\section{Cerebral Venous Thrombosis: Continental Characteristics and Disparity}

Laith Maali, Sheema S. Khan, Mahmoud M. Ismail, Rhys R. Brooks, Vishnumurthy V. Shushrutha Hedna.

The University of New Mexico, Albuquerque, NM, USA.

\section{Introduction:}

Cerebral venous thrombosis (CVT) usually accounts for $<1 \%$ of all strokes. Global disparity and diversity in their demographics, etiology, clinical features, radiological presentation, and mortality have not been previously explored.

\section{Methods:}

A systematic search was performed for publications in PubMed using key words "Cerebral venous thrombosis", "Cerebral vein thrombosis" and "Cortical vein Thrombosis". A total of 600 relevant studies were abstracted with strict selection criteria and a total of 7048 patients' data were used for the final analysis. Linear correlation was used for our descriptive analysis.

\section{Results:}

Cases reported were Europe-3152, Asia-2722, North America-852, Africa-122, Australia-121 and South America-79. Overall Male to female ratio was 1:2.2, among clinical characteristics headache was the most common symptom and hematological factors were the most common etiology. Location of the thrombosis was described mostly in the transverse sinus. Intercontinental differences in relation to demographics, etiology, clinical features, radiological presentation, and mortality were identified.

\section{Conclusions:}

CVT can have significant disparity in their demographics, etiology, clinical features, radiological presentation, and mortality when compared from one continent to another. It is important for the worldwide physicians to recognize these differences and to follow the most recent guidelines, diagnostic methods and treatment to insure the best outcome and prognosis. 


\title{
Poster 151
}

\section{Occurrence and Timeliness of Goals of Care Discussion in the Neurocritical Care Unit}

\author{
Aarti Sarwal', Kristopher K. Dixon ${ }^{1}$, Mollie M. Canzona ${ }^{2}$, Michael M. Hyde ${ }^{3}$, Shayn S. Martin ${ }^{1}$, David D. \\ Bowton ${ }^{1}$, John J. Wilson ${ }^{1}$, Allison A. Brashear'. \\ ${ }^{1}$ Wake Forest School of Medicine, Winston Salem, NC, USA, ${ }^{2}$ Wake Forest University, Department of \\ Communication, Winston Salem, NC, USA, ${ }^{3}$ Wake Forest University, Department of Communication, \\ Winston Salem, NC, USA.
}

\section{Introduction:}

Timely communication is critical for high quality care in the intensive care unit(ICU). Published literature in medical/surgical ICUs quotes up to $81 \%$ of patient caregivers receive prognostic information with mean prognostic interval $1.7 \pm 2.8$ days since ICU admission Prognostication in acute neurological injuries is challenging and uncertainty may delay communication. We assess occurrence and timeliness of goals of care communication in a Neurocritical Care unit

\section{Methods:}

Prospective observational study by surveying nurses in a 22 bed Neurocritical care unit in tertiary level care academic medical center over a 4 week period. Data was also collected during daily morning multidisciplinary huddle and verified by verbally surveying the nurses.

\section{Results:}

Survey results were analyzed for 97 patients and 185 patient encounters. In 99.4\% encounters, the nurses felt the patient's treatment plan matched patient-centered goals of care In $79.3 \%$ encounters, a provider family discussion had occurred in the last 24 hours. Within the 1 st 24 hours of ICU admission, $40.2 \%$ patients were identified to need goals of care discussion in the multidisciplinary huddle, only $61.5 \%$ had such a discussion. For patients needing goals of care addressed, a discussion occurred on an average $1.3 \pm 0.6$ days since ICU admission. Dichotomized by age, $73.7 \%$ patients younger than 65 years old had a discussion, if one was needed, while only $50 \%$ older than 65 years had one. When dichotomized by gender, $66.7 \%$ of males and $81.4 \%$ of females had a provider discussion. $68.4 \%$ females compared to $55 \%$ of males received a discussion on goals of care if identified as needed within 1 st 24 hours of ICU admission.

\section{Conclusions:}

Our data shows timely communication of goals of care in the Neurocritical care unit with a mean time comparable to published literature. However, there appear to be demographic disparities that warrant further research. 
Poster 152

Cerebral Vasomotor reactivity reflect prognosis after cardiac arrest

SungEun Lee.

Ajou University School of Medicine / Department of Neurology, Suwon, Korea, Republic of.

\section{Introduction:}

Neurological prognostication after cardiac arrest is a difficult problem. Since several studies reported good effect of target temperature management (TTM), prognostication after cardiac arrest was delayed and became complex. Recently, some reports presented that impaired cerebral autoregulation was correlated with neurologically poor outcome. The aim of this study was to determine whether vasomotor reactivity (VMR) test by transcranial doppler (TCD), reflecting cerebral hemodynamic status, affected accuracy of neurological prognostication in post cardiac arrest patients.

\section{Methods:}

Since January 2016, 8 patients were enrolled after cardiac arrest. Patients who performed VMR test during TTM period were included and patient with unstable vital sign or malignant findings in brain CT, such as massive subarachnoid hemorrhage or severe brain edema, or poor temporal windows. Primary outcome was cerebral performance category scale (CPC) at discharge. VMR test used breath-holding method during 40 seconds. Carbon dioxide level was checked by capnometer and arterial blood gas analysis. Other conventional prognostication test, such as EEG, SEP, et al., was performed after 72 hours from rewarming time. We divided patients between good (CPC 1-2) and poor (CPC 3-5) outcome group and compared results from prognostic test between two groups.

\section{Results:}

As previous study, pupuil light reflex, corneal reflex, presence of N20 at median nerve sensory evoked potential, and electroencephalography after 72 hours from rewarming time were presented favorable results in good outcome group. $(p<0.001)$ VMR during breath-holding technique during TTM period also was more increased in good outcome group at right $(41.10 \pm 1.15 \%$ vs. $12.17 \pm 3.01 \%, p<0.001)$ and left $(36.47 \pm 6.48 \%$ vs. $7.23 \pm 10.65 \%, p<0.001)$ middle cerebral arteries.

\section{Conclusions:}

The present study shows that vasomotor reactivity is preserved in patients with neurological good outcome. To evaluating cerebral hemodynamic status by VMR test seems to be useful tool for early prognostication after cardiac arrest. 


\section{Poster 153}

\section{Can a Standardized Advanced Practice Provider (APP) Orientation Process Transition New APP Skills Beyond the Level of the Neuron?}

Michelle L. Lozano, Susan S. Yeager.

The Ohio State University Wexner Medical Center, Columbus, OH, USA.

\section{Introduction:}

As the numbers and opportunities for Advanced Practice Providers (APPS) in Neurocritical Care Units (NCCUs) has increased, the integration of these providers into the health care setting has become a greater challenge. Currently no data exists to support how training and skill integration should occur for APPs starting careers in the NCCU. The purpose of this poster is to provide data of APPs knowledge and comfort levels before and after completion orientation.

\section{Methods:}

This prospective, pre and post observational study was sent to 10 newly hired NCCU APPs within an Academic Medical Center. A one hundred- item survey was created to evaluate self-reported experience and comfort levels with specific skills, diagnoses, and management of issues common to NCCUs. Baseline data was collected from each APP. Next, APPs were integrated into the NCCU utilizing a three month orientation program which fused a series of didactic, simulated, and precepted experiences. After orientation, APPs were asked to recomplete the survey tool. Student's $t$ test statistics were utilized to compare before and after experience and comfort levels with items identified as necessary to perform in the NCCU APP role.

\section{Results:}

In progress

\section{Conclusions:}

As utilization of APPs in the NCCU becomes more prevalent, integration processes need developed to match APP entry level knowledge while enabling quick progression into high quality, independent practice. A structured approach enables identification of high priority areas to assist with initial and ongoing training needs. This study indicates that APPs were able to improve NCCU related knowledge and comfort levels. Results indicate that further education and exposure to items such as neurologic imaging may be helpful. Limitations of this study include subjective data from a small, self-reported, single institutional sample. Further research of larger, more diversified sample representation is needed to validate whether these results can be generalized to other NCCUs. 
Poster 154

\section{Early Evaluation of post-arrest injury and tailored TTM}

Yasuhiro Kuroda, Kenya K. Kawakita, Toru T. Hifumi.

Department of Emergency Medicine, Kagawa University, Miki, Japan.

\section{Introduction:}

Brain damage after return of spontaneous circulation (ROSC) varies among studies and patients despite their comatose status (i.e., lack of meaningful response to verbal commands), because of the absence of an established modality enabling proper evaluation. Evaluation of brain injury after ROSC is needed for the determination of the inclusion criteria of neurocritical care, especially of targeted temperature management.

\section{Methods:}

Literatures are reviewed and summarized.

\section{Results:}

The association between admission Glasgow Coma Score (GCS) motor score and neurologic outcome remains unknown in comatose cardiac arrest survivors. We found that GCS motor scores immediately after ROSC (day 0 ) is an independent predictor of good neurologic outcome at 90 days in patients sustaining out-of-hospital cardiac arrest who receive therapeutic hypothermia: GCS motor score 1, $\mathrm{n}=130$ (52.2\%); score 2-3, $n=23$ (76.7\%); score 4-5, $n=20$ (87.0\%), $P<0.01$ (Hifumi 20152201 ). Recently no significant differences of neurologic outcome at 30 days after hospital admission was observed between mild therapeutic hypothermia and control in the subgroup of GCS Motor score 5 or 6 . These data show that initial GCS motor score examination immediately after ROSC can at least provide baseline objective prognostic data for decisions by healthcare professionals.

\section{Conclusions:}

Neurological signs such as GCS, brain stem reflex, respiratory status, and degree of shivering are potential variables that can be incorporated into a predictive model for a more precise evaluation of brain injury in cardiac arrest survivors undergoing TTM. Effect of targeted temperature management should be evaluated depending on the brain injury in PCAS. 


\section{Poster 155}

\section{Endovascular Therapy in Pediatric Arterial Ischemic Stroke}

Cydni N. Williams, Jennifer J. Wilson.

Oregon Health and Science University, Department of Pediatrics, Portland, OR, USA.

\section{Introduction:}

Though standard care for adult stroke, little is known about endovascular therapy (ET) for pediatric arterial ischemic stroke (AIS). We aimed to describe population-level estimates of ET utilization in pediatric AIS, and explore demographic and clinical characteristics, associated interventions, and outcomes.

\section{Methods:}

Retrospective cohort analysis of the Kids' Inpatient Database evaluated ET utilization in children with AIS and age $>28$ days, identified by diagnosis and procedure codes. Analyses were weighted for national estimates and compared with Chi-square and t-tests.

\section{Results:}

Among 3184 pediatric AIS patients, $38(1 \%)$ received ET. Anterior circulation occlusions were seen in $89 \%$ of ET patients. ET patient age ranged $2-20$ years, with $78 \% \geq 11$ years. Cardiac factors were most common in both groups $(42 \% \mathrm{ET}$ versus $28 \%, \mathrm{p}=.07)$. Other risk factors were similar. Paresis $(64 \% \mathrm{ET}$ versus $30 \%, p<.001)$ was more common and seizure was less common $(11 \% \mathrm{ET}$ versus $31 \%, p=.004)$ in ET patients. Average age was higher with ET (14 versus 10 years, $p<.05)$. Other patient demographics, hospital characteristics, and critical care procedures were similar. Thrombolytic agents (tPA) were common with ET (51\% ET versus $2 \%$ overall). Intracranial hemorrhage was similar ( $14 \%$ ET versus $8 \%$, $p=.12$ ), and varied by tPA (22\% ET with tPA, 10\% tPA only, 7\% ET only, $8 \%$ neither). There was a nonsignificant trend toward poor outcome (death, discharge to nursing facility, tracheostomy, or gastrostomy) with ET (45\% ET versus $30 \%, p=.07$ ). Among AIS patients $\geq 5 y$ ears with paresis, significant association was seen between poor outcome and ET (68\% ET versus $39 \%, p=.004)$.

\section{Conclusions:}

ET in pediatric AIS is uncommon, utilized mostly in older children and those with paresis. Though hemorrhage was uncommon, this data suggests caution with ET and tPA combination. Associations between ET and poor outcome may reflect disease severity bias. More research on outcomes with ET in pediatric AIS is needed. 


\title{
Poster 156
}

\section{Epidemiology And Outcomes Of Children With Diabetic Ketoacidosis Presenting With Cerebral Edema}

\author{
Luis P. Lee, Michael M. Leoncio, Balagangadhar B. Totapally.
}

Nicklaus Children's Hospital / Pediatric Critical Care Department, Miami, FL, USA.

\section{Introduction:}

Diabetic ketoacidosis (DKA) is the most common cause of hospitalization of children with type 1 diabetes and cerebral edema is the most serious complication leading to morbidity and mortality. We queried a nationally representative database to determine epidemiologic data of cerebral edema in children with DKA.

\section{Methods:}

An analysis of the Healthcare Cost and Utilization Project's Kids Inpatient Database for the year 2012 was performed. The database was filtered using ICD-9 diagnosis codes for DKA $(250.10,250.11,250.12$, 250.13 ) and cerebral edema (348.5) from the age of 1 month to 20 years. We examined these hospitalizations by age, gender, race/ethnicity, regions of the United States, comorbid diagnosis, clinical procedures, outcome and mortality rates. Sample weighing was employed to produce national estimates. Chi-square test, Mann Whitney $U$ test and binary regression analysis were performed using SPSS to analyze the data.

\section{Results:}

A total of 38,972 patients with DKA were discharged during 2012. Females were $55 \%$. Racial distribution includes, whites $58 \%$, blacks $22 \%$ and Hispanics $14 \%$. The median age was 16 (IQR: 12-19) years. Cerebral edema was present in $188(0.5 \%)$ children. The overall mortality rate was $0.08 \%$, but the mortality rate in children who developed cerebral edema was higher at 6.7\% (OR: 151; 95\% Cl: 73-310). Mortality was higher in children who had a major operative procedure $(1.13 \%$ vs $0.07 \%$; OR $16,95 \% \mathrm{Cl}$ : $6-45)$ and in those with Medicaid compared to private insurance $(0.11 \%$ vs $0.04 \% p=0.031)$ and lower in whites $(0.04 \%)$ compared to blacks $(0.13 \%)$ or Hispanics $(0.2 \%)$. Length of stay, number diagnoses, number of chronic conditions, and hospital charges were significantly higher among non-survivors but there was no difference in the age.

\section{Conclusions:}

The overall mortality rate in children admitted with DKA is $0.08 \%$. Cerebral edema prevalence is $0.5 \%$ and it increases mortality significantly. 


\section{Poster 157}

\section{Correlation of Regional Cerebral Oxygenation with Cerebral Perfusion Pressure in Children with Intracranial Hypertension due to acute Central Nervous System (CNS) Infections - A Prospective Observational Study}

Mullai Baalaaji, Sunit S. Singhi, Muralidharan M. Jayashree, Arun A. Bansal.

Pediatric Intensive Care Unit, Department of Pediatrics, PGIMER, Chandigarh, India.

\section{Introduction:}

Near-Infrared Spectroscopy (NIRS), a non-invasive modality to measure regional cerebral oxygenation (rSO2), is being increasingly used to monitor cerebral tissue oxygenation. We studied relationship of rSO2 with cerebral perfusion pressure (CPP) and intracranial pressure (ICP) in children with acute CNS infections to determine if rSO2 could be used as non-invasive surrogate for CPP.

\section{Methods:}

In a prospective observational study we enrolled 31 children, aged $<12$ years, with raised ICP due to acute CNS infections after approval by Institutional Ethics Committee. They were monitored simultaneously for rSO2 of both frontal-lobes (INVOS Cerebral/Somatic Oximeter, Model-5100C, Covidien-IIC), invasive blood pressure, and ICP using intraparenchymal fibre-optic catheter (Codman). Linear trends and correlation coefficients were used to define relation of rSO2 with ICP and CPP.

\section{Results:}

A total of 3378 paired values of rSO2, ICP and CPP were analysed. The linear trends during the first 72 hours revealed no significant correlation between changes in rSO2 and changes in ICP and CPP from baseline (R2 $=0.007,0.012$ for ICP and CPP respectively). However, the trend was not uniform $-48 \%$ patients had no correlation between rSO2 and CPP, $29 \%$ showed a positive correlation and $23 \%$ showed a negative correlation. Subgroup analysis revealed that strength of correlation between rSO2 and ICP/CPP were stronger during the time points when ICP was $>30 \mathrm{mmHg}\left(\mathrm{rSO}{ }^{*} \mathrm{ICP} \rho=-0.421, \mathrm{p} 20\right.$ $\mathrm{mmHg}$ and normal CPP were 6.1(3.4-11.1,p50\% respectively.

\section{Conclusions:}

rSO2 has complex interaction with ICP and CPP; the changes in ICP and CPP could not predict changes in rSO2. However, the odds for normal CPP was significantly higher when rSO $2>50 \%$ and this cut-off could be used as a non-invasive target for age appropriate CPP. 


\title{
Poster 158
}

\section{Enteral Nutrition Initiation in Children Admitted to the Pediatric Intensive Care Unit after Traumatic Brain Injury}

\author{
Binod Balakrishnan ${ }^{1}$, Mahua M. Dasgupta ${ }^{2}$, Pippa P. Simpson ${ }^{2}$, Katherine K. Flynn-O'Brien ${ }^{3}$, Sheila S. \\ Hanson $^{2}$. \\ ${ }^{1}$ Medical College of Wisconsin, Milwaukee, WI, USA, ${ }^{2}$ Medical College of Wisconsin, Milwaukee, WI, \\ USA, ${ }^{3}$ University of Washington, Department of Surgery, Seattle, WA, USA.
}

\section{Introduction:}

Earlier feeding results in improved outcomes in adults with severe traumatic brain injury (TBI) and in the overall pediatric intensive care unit (PICU) population. Current practices of nutrition initiation in children with TBI are not well described. This multicenter study evaluated timing and factors associated with nutrition initiation in children admitted to PICUs with TBI. We hypothesize that severely brain injured patients would have a delay in initiation of enteral nutrition.

\section{Methods:}

We retrospectively analyzed the multicenter Pediatric Trauma Assessment and Management Database (PTAM) from 2013. Patients with severe TBI were defined as Glasgow Coma Scale (GCS) <9 with Abbreviated Injury Score (AIS) head $\geq 2$. Time to initiation of enteral nutrition in this group was compared to those with GCS $\geq 9$. Demographics, outcomes, injury/illness severity scores, presence of abdominal injury, abdominal procedures were compared between the two groups. Chi square and Fisher exact tests were used for dichotomous variables; non-parametric tests were used for continuous variables.

Multivariable regression analysis with a stepwise procedure was performed to ascertain the best set of variables associated with delayed initiation of enteral nutrition.

\section{Results:}

Of 423 patients admitted to the five PTAM PICUs with severe TBI, $285(67 \%)$ were fed $<24$ hours from admission. Patients with GCS $<9$ were fed a median 45.1 hours from admission (IQR 22.0-72.6) compared to 10.3 hours (IQR 3.8-17.7) for those with GCS $\geq 9$. Site, abdominal injury/procedures, bowel regimen, higher injury and illness severity scores and lower minimum GCS were significantly associated with feeding initiation $>24$ hours. On multivariable analysis, scheduled bowel regimen, higher PRISM score and lower minimum GCS were significantly associated with nutrition initiation $>24 \mathrm{hrs}$.

\section{Conclusions:}

Lower GCS is independently associated with delayed initiation of enteral nutrition in children with TBI, independent of severity of injury or abdominal injury. 


\title{
Poster 159
}

\section{Outcome In Refractory and Super-Refractory Status Epilepticus In Children}

\author{
Jennifer C. Erklauer ${ }^{1}$, James J. Owens ${ }^{2}$, Angus A. Wilfong ${ }^{3}$, Graf G. Jeanine ${ }^{4}$, Mona M. McPherson ${ }^{4}$, \\ Brady B. Moffett ${ }^{5}$, Laura L. Loftis ${ }^{4}$.
}

\begin{abstract}
${ }^{1}$ Baylor College of Medicine and Texas Children's Hospital/Critical Care, Neurology, Houston, TX, USA, ${ }^{2}$ University of Washington/Pediatrics and Neurology, Seattle, WA, USA, ${ }^{3}$ Baylor College of Medicine and Texas Children's Hospital/Pediatrics, Neurology, Houston, TX, USA, ${ }^{4}$ Baylor College of Medicine and Texas Children's Hospital/Pediatric Critical Care, Houston, TX, USA, ${ }^{5}$ Baylor College of Medicine and Texas Children's Hospital/Pharmacy and Cardiology, Houston, TX, USA.
\end{abstract}

\section{Introduction:}

Refractory status epilepticus is persistent seizure activity despite treatment with one first-line and one second-line anti-epileptic medication, while seizure activity $>24$ hours is considered super-refractory. Functional outcome for children with these conditions is not well defined. This study describes functional outcome for children with refractory and super-refractory status epilepticus proposing that prognosis will be variable with high mortality. Survivors will be more likely to experience functional neurologic deficits and technology dependence.

\section{Methods:}

This retrospective chart review evaluated children age $0-18$ years who received pentobarbital infusion at Texas Children's Hospital pediatric intensive care unit from 2004-2015 for status epilepticus. Outcome was defined using pediatric cerebral performance category score (PCPC) at time of discharge and at the most recent clinical evaluation per the medical record. Additional measures included mortality, need for medical technology (tracheostomy or gastrostomy tube), seizure burden, and number of seizure medications at discharge.

\section{Results:}

42 children met inclusion criteria. In-hospital mortality was $31 \%$, secondary to withdrawal of support $(54 \%)$, brain death $(31 \%)$, or cardiac arrest $(15 \%)$. Highest mortality occurred in acute hypoxic ischemic injury $(p=0.04)$. Of survivors, $39 \%$ returned to baseline PCPC at discharge while $46 \%$ demonstrated decline in function $\geq 2$ PCPC categories at discharge. 7 children required tracheostomy and 7 children underwent gastrostomy tube placement. Seizures persisted at discharge for most patients with no prior history of seizure, however, the majority of patients with known epilepsy returned to baseline seizure frequency. Most children required additional home seizure medications. Long-term follow-up was documented for 22 survivors up to 9 years after discharge. $32 \%$ demonstrated improved PCPC and $18 \%$ showed decline including 3 additional deaths.

\section{Conclusions:}

Mortality in this population was high. Functional outcome in survivors was variable. Some children returned to neurologic baseline by time of discharge and for those who did not, continued functional improvement was possible over time. 


\title{
Poster 160
}

\section{MRI Biomarkers of Functional Outcome after Severe Pediatric TBI: A Feasibility Assessment}

\author{
Peter Ferrazzano ${ }^{1}$, Bedda B. Rosario ${ }^{2}$, Stephen S. Wisniewski ${ }^{2}$, Andrew A. Alexander ${ }^{3}$, Michael M. Bell ${ }^{4}$.
}

${ }^{1}$ University of Wisconsin Department of Pediatrics, Madison, WI, USA, ${ }^{2}$ University of Pittsburgh Department of Epidemiology, Pittsburgh, PA, USA, ${ }^{3}$ University of Wisconsin Department of Medical Physics, Madison, WI, USA, ${ }^{4}$ University of Pittsburgh Department of Critical Care Medicine, Pittsburgh, PA, USA.

\section{Introduction:}

Purpose: Establish the feasibility of conducting a neuroimaging study of severe pediatric $\mathrm{TBI}$ as an ancillary study to the ongoing ADAPT Trial, with adequate power to identify biomarkers of specific neurocognitive dysfunctions.

\section{Methods:}

Methods: We conducted a survey of ADAPT site-PIs to determine imaging practices in severe TBI patients and interest in participating in an ancillary neuroimaging study. We subsequently determined the incidence and timing of MRI scan in subjects enrolled in the ADAPT Trial at 10 sites.

\section{Results:}

Results: 25/48 ADAPT sites completed the survey. Most respondents (22/25) indicated that they obtain an MRI scan in over half of children with severe TBI, with 9/25 sites reporting MRI scanning in $>95 \%$ of their severe TBI patients. Regarding timing of MRI, 20/25 respondents reported that severe TBI patients typically have an MRI obtained during the first 2 weeks post-injury. In addition to standard anatomic imaging, MR sequences obtained "often or always" included: diffusion-weighted (22/25), susceptibilityweighted (17/25), T2*-weighted gradient recall echo (16/25), diffusion tensor imaging (9/25), and perfusion-weighted imaging (7/25). Only $8 / 25$ sites reported use of a standardized MRI protocol for pediatric TBI patients. In a retrospective analysis at 10 ADAPT sites, we found that 90/130 ADAPT subjects received an acute MRI within 30 days post-injury. Fifteen ADAPT sites, accounting for over $50 \%$ of ADAPT enrollment, committed to recruit ADAPT subjects for a non-sedated MRI scan at one year post - TBI.

\section{Conclusions:}

Conclusion: Collection of 400-600 acute MRI scans from the 1000 subjects enrolled in ADAPT to study associations between acute MRI findings and functional outcome is potentially feasible. Allowing for $20 \%$ mortality and 50\% recruitment rate, recruitment of 100-150 ADAPT subjects from 15 ADAPT sites for a follow-up MRI to study relationships between advanced MRI measures and neurocognitive function is potentially feasible and would represent the largest such study conducted to date. 


\title{
Poster 161
}

\section{Antimicrobial prescribing practices and antibiotic resistance following neurosurgical drain placement: A single-center observational study}

\author{
Andrea J. Passarelli, Hasan H. Alhasani.
}

Christiana Care Health System Department of Pharmacy, Newark, DE, USA.

\section{Introduction:}

The use of systemic antibiotics for the duration of neurosurgical drain placement has not been associated with reduced rates of drain related infection (DRI) and may contribute to the development of antimicrobial resistance and Clostridium difficile infection (CDI). We sought to describe antimicrobial prescribing practices, incidence of DRI, and development of antimicrobial resistance and CDI after neurosurgical drain placement at our institution.

\section{Methods:}

This was a single center study including adult patients status post ventriculostomy or craniotomy/craniectomy with drain placement from 6/1/14-5/31/15. Exclusion criteria were: active infection/antibiotic escalation or death within 48 hours of drain placement, neutropenia, placement of ventriculoperitoneal shunt or Ommaya reservoir, and use of an antibiotic impregnated drain. Bacterial cultures and $\mathrm{C}$. difficile PCR during the index admission and 90 days post-discharge were collected. Antibiotic resistance was defined as an organism resistant to the prophylactic agent. Prolonged prophylaxis was defined as antibiotics continued for $>48$ hours after drain placement.

\section{Results:}

Eighty-one patients with 107 drains were included. The median duration of prophylaxis was 3.3 days and cefazolin was most commonly prescribed agent (85\%). Three of 48 patients with EVDs developed DRI. No risk factors for DRI were identified and there was no difference in incidence of DRI when using prolonged vs. perioperative prophylaxis. Of non-DRIs $60 \%$ were resistant to the prophylactic agent used. E. coli, K. oxytoca, and S. aureus had higher rates of resistance to cefazolin compared to our institutional antibiogram, although not statistically significant. No patients developed CDI.

\section{Conclusions:}

The use of prolonged prophylaxis was not associated with a reduced reduction in DRI. Most bacterial isolates were resistant to the prophylactic agent used. We suggest that antibiotic prophylaxis for neurosurgical drain placement be limited to one preoperative dose within 60 minutes of the procedure with appropriate intraoperative dosing to reduce the risk of antimicrobial resistance. 


\section{Poster 162}

\section{Levetiracetam for seizure prophylaxis after cerebrovascular surgery}

Lynn V. Do ${ }^{1}$, Crystal C. Zhou ${ }^{2}$, Crystal C. Sau ${ }^{1}$, Michael M. Trillanes ${ }^{1}$, Nirav N. Shah ${ }^{3}$, Scott S. Caganap ${ }^{3}$, Lisa L. Hannegan ${ }^{4}$, Marlene M. Burt ${ }^{4}$, Michael M. Lawton ${ }^{4}$.

${ }^{1}$ University of California San Francisco, Department of Pharmacy, San Francisco, CA, USA, ${ }^{2}$ University of California San Francisco, School of Pharmacy, San Francisco, CA, USA, ${ }^{3}$ University of California San Francisco, Department of Neurology, San Francisco, CA, USA, ${ }^{4}$ University of California San Francisco, Department of Neurological Surgery, San Francisco, CA, USA.

\section{Introduction:}

Pharmacologic seizure prophylaxis after cerebrovascular surgery is not well-supported by literature as the risk of early postoperative seizures is low. Seizure risk in aneurysmal subarachnoid hemorrhage (aSAH) has been described based on preoperative presentation, but other clinical factors are heterogeneous and unclear. Despite the lack of evidence, levetiracetam (LEV) continues to be initiated at many institutions. The objectives of this study are to evaluate our institution's practice for initiating seizure prophylaxis postoperatively and establish a standard of care.

\section{Methods:}

Adult patients who underwent cerebrovascular surgery from August 2013 to July 2015 were screened for study inclusion. Patients who received LEV postoperatively were compared to those who did not receive LEV. Clinical seizures and data were obtained from retrospective review of electronic medical records. The primary outcome was seizure occurrence in the first 7 days after surgery. Secondary outcomes included characteristics or potential risk factors that led to seizure occurrence or prompted LEV initiation.

\section{Results:}

Of the 160 patients included in the study, there were 115 in the no LEV group and 45 in the LEV group. Two seizures occurred in the no LEV group while no seizures occurred in the LEV group (2 vs 0 , $p=0.373$ ). There were no differences between surgery type, intraoperative blood loss or proportion of aSAH. Of the 25 patients with aSAH, $64 \%$ were not on LEV and 1 seizure occurred. Of 19 patients with intraparenchymal or intraventricular extension, $63 \%$ were not on LEV. Average length of stay was prolonged for the LEV group (12 vs 7 days, $p<0.001$ ).

\section{Conclusions:}

The majority of patients did not receive LEV postoperatively and there was no difference in seizure occurrence. A pattern for initiating LEV based on seizure risk factors was not identified at our institution. Developing a standardized approach for initiating LEV may decrease variability in practices and streamline postoperative care. 


\title{
Poster 163
}

\section{Post-operative pain control after craniotomy: A meta-narrative review}

\author{
Jonathan J. Rasouli ${ }^{1}$, Molly M. Ratner ${ }^{1}$, Alexander A. Chartrain ${ }^{2}$, Stephan S. Mayer ${ }^{1}$, Errol E. Gordon ${ }^{1}$, \\ Neha N. Dangayach".
}

\author{
${ }^{1}$ Mount Sinai Health System, Department of Neurosurgery, New York, NY, USA, ${ }^{2}$ Department of \\ Neurosurgery, Mount Sinai Health System, New York, NY, USA.
}

\section{Introduction:}

Craniotomy is commonly performed for the treatment of a variety of conditions including brain tumors, aneurysms, and vascular malformations. Despite significant advances in the quality and efficacy of neuroanesthetic care, there are no evidence-based guidelines for the management of post-operative pain after craniotomy. Uncontrolled post-operative pain has been directly linked to increased length of hospital stay, increased hospital care costs, and poor health-related quality of life. Due to the lack of evidencebased clinical decision rules, clinicians often rely on institutional or expert-based opinions to guide their decision-making. With the recent release of the Center for Medicare/Medicaid Services (CMS) statement on opioid use, there an urgent need to evaluate existing pain management protocols. Hence, we conducted a meta-narrative to evaluate heterogeneity in current practices regarding management of postoperative pain after craniotomy.

\section{Methods:}

A meta-narrative review was performed utilizing the MEDLINE/PUBMED search engine with the search terms "pain" and "craniotomy". A total of 344 articles and 16 systematic reviews were resulted. Inclusion criteria were studies from 2004-2016, randomized controlled trials, retrospective studies, systematic reviews, case reports, case series published in English were included. Of these, 230 articles and 7 systematic reviews were included in the final analysis.

\section{Results:}

There is a paucity of randomized controlled trials to develop evidence based peri-operative pain management protocols in craniotomy patients. There is evidence to suggest that scalp infiltration with local anesthetic may improve post-operative pain scores immediately after surgery. The perioperative use of nonsteroidal anti-inflammatory medications may improve pain scores without a subsequent increase in the risk of hemorrhage.

\section{Conclusions:}

Management of post-operative pain after craniotomy remains a challenging problem for clinicians and patients. There is an urgent need to conduct well designed randomized controlled trials to guide perioperative pain management in craniotomy patients and to use opioid sparing techniques for improving patient outcomes. 


\title{
Poster 164
}

\section{Blood flow and cEEG changes during symptomatic plateau waves: two cases}

\author{
Margaret Huynh, $D O^{1}$, Natalie N. Kreitzer, $M D^{2}$, Brandon B. Foreman, $M D^{1}$. \\ ${ }^{1}$ University of Cincinnati, Department of Neurology \& Rehabilitation Medicine, Cincinnati, OH, USA, ${ }^{2}$ \\ University of Cincinnati, Department of Emergency Medicine, Cincinnati, OH, USA.
}

\section{Introduction:}

Symptomatic plateau waves are characterized by paroxysmal neurological symptoms suggestive of elevated intracranial pressure such as depressed level of consciousness, pupillary dilatation, and dysautonomia in a patient with an intracranial mass lesion. Clinically, these episodes may be mistaken for seizures, syncope, or new brain injury. Noninvasive cerebral blood flow can be measured using ultrasound-tagged infrared spectroscopy; continuous EEG is sensitive to changes in blood flow. We report two patients without invasive intracranial pressure monitoring who demonstrated changes in blood flow and EEG during symptomatic plateau waves.

\section{Methods:}

Case Series.

\section{Results:}

Case 1 was a 24 year-old man with fungal ventriculomeningitis. After a prolonged hospital course, he developed an entrapped 4th ventricle and began to experience periods of complete unresponsiveness with anisocoria, clonus, and tachy- or bradycardia lasting between 10 and 20 minutes. Episodes resolved after decompression and ventricular stent placement. Case 2 was a 49 year-old woman with intraventricular meningioma who underwent partial resection with entrapment of the right lateral ventricle. On post-operative day 7 she developed multiple episodes of unresponsiveness, diaphoresis, clonus, tachy- or bradycardia lasting 10 to 20 minutes, culminating in a persistent episode requiring urgent craniotomy. In both patients, cEEG was started to assess for seizures and ORNIM device was used to characterize blood flow. In each, symptomatic plateau waves were accompanied by decreased blood flow, followed by attenuation of faster frequencies on the cEEG.

\section{Conclusions:}

Symptomatic plateau waves may be characterized noninvasively by using surface measurements of blood flow and cEEG. This case series demonstrates that decreases in bifrontal blood flow lead to depressions in cEEG during these symptomatic plateau waves. Noninvasive measurement of blood flow in conjunction with cEEG provides an adjunct to invasive ICP monitoring in patients with mass lesions at risk for developing symptomatic plateau waves. 


\title{
Poster 165
}

\section{Hemodynamic properties of SAGE-547 in patients with super-refractory status epilepticus: results from a phase $1 / 2$ clinical trial}

\author{
Stephen S. Kanes ${ }^{1}$, Shane S. Raines ${ }^{2}$, Helen H. Colquhoun ${ }^{1}$. \\ ${ }^{1}$ Sage Therapeutics, Inc., Cambridge, MA, USA, ${ }^{2} 2 b$ Analytics, Wallingford, PA, USA.
}

\section{Introduction:}

An open-label phase $1 / 2$ clinical study (547-SSE-201) evaluated the safety, pharmacokinetics, and efficacy of SAGE-547, a proprietary formulation of allopregnanolone, in patients with super-refractory status epilepticus (SRSE). Overall, $77 \%$ (17/22) of evaluable patients were successfully weaned off thirdline agents (TLAs) while SAGE-547 was being administered; $64 \%$ experienced $\geq 1$ serious adverse event (none attributed by the Safety Committee to SAGE-547). Post-hoc analyses evaluated the pharmacological effects of SAGE-547 and the effect of SAGE-547 administration in the context of multiple antiepileptic drugs (AEDs), pressors, and TLAs. Here we examine the hemodynamic properties of SAGE547 in the study patients, with the goal of further understanding the clinical context of SAGE- 547 administration in this critically ill population.

\section{Methods:}

Patients $\geq 2$ years old with SRSE and receiving continuous intravenous infusion of $\geq 1$ TLA for seizure or burst suppression were eligible if breakthrough seizures occurred during a wean attempt from or during maintenance of the TLA. Key exclusion criteria were anoxic brain injury and very short life expectancy. At enrollment, patients received an average of 3.0 AEDs and 1.4 TLAs with an average status epilepticus duration of 9.2 days. Hemodynamic measurements (heart rate, systolic and diastolic blood pressure) were collected at screening, pre-dose, during SAGE-547 treatment (15, 30, 45, 60 minutes; 2, 4, 8, 24, $48,72,96,120$ hours) and follow-up (6, 7, 8, 15, 22, 29 days). Pharmacokinetic/pharmacodynamic (PK/PD) modeling based on physiological parameters (heart rate, blood pressure) and need for pressors was examined.

\section{Results:}

Twenty-five patients received treatment with SAGE-547. During the study, mean changes in hemodynamic parameters from baseline were limited, both for patients receiving the standard $(n=19$ patients) and high ( $\mathrm{n}=6$ patients) SAGE-547 dose.

\section{Conclusions:}

Regarding hemodynamic parameters, SAGE-547 was well tolerated in the SRSE patients studied, suggesting for further study that SAGE-547 may not elicit immediate or sustained hemodynamic changes in SRSE patients. 
Poster 166

\title{
Duration of Seizure Prophylaxis after Subarachnoid Hemorrhage (DOPAST): Results of a Randomized Comparative Effectiveness Trial
}

\author{
Theresa Human ${ }^{1}$, Michael M. Diringer $^{2}$, Gregory G. Zipfel ${ }^{3}$, Michael M. Chicoine ${ }^{3}$, Ralph R. Dacey ${ }^{3}$, \\ Michelle M. Allen', Rajat R. Dhar'.
}

\author{
${ }^{1}$ Barnes-Jewish Hospital / Washington University, St. Louis, MO, USA, ${ }^{2}$ Washington University, \\ Department of Neurology, St. Louis, MO, USA, ${ }^{3}$ Washington University, Department of Neurosurgery, St. \\ Louis, MO, USA.
}

\section{Introduction:}

Seizures occur in $10-20 \%$ of patients with subarachnoid hemorrhage (SAH), predominantly in the acute phase. Anticonvulsant prophylaxis remains controversial, with some studies suggesting longer exposure is associated with worse outcomes. A comparison of a short course of phenytoin to historical controls treated for longer duration suggested equal effectiveness, but a similar study with levetiracetam (LEV) at our institution found a higher seizure rate with shorter duration prophylaxis. The objective of this study was to compare strategies for seizure prophylaxis after aneurysmal SAH.

\section{Methods:}

This was a prospective single-center randomized open-label trial of a short (3-day) course of LEV (1000 $\mathrm{mg}$ BID) vs. extended course (until hospital discharge). The primary outcome was in-hospital seizure after randomization. Secondary outcomes included incidence of adverse drug reactions, rate of drug discontinuation, and functional/cognitive outcomes.

\section{Results:}

$84 \mathrm{SAH}$ patients were randomly allocated to the two groups when the trial as terminated due to slow enrollment; baseline characteristics were comparable. In-hospital seizures occurred in 3 of $35(9 \%)$ of the short-duration LEV group vs. 1 of $49(2 \%)$ of the extended LEV group(OR 4.5, 95\% 0.5-50, $\mathrm{Cl} p=0.2)$. All study seizures occurred beyond 3-days (median time 9 days). The Kaplan-Meier seizure-free curves diverged at approx. 10 days $(p=0.16$ by log-rank test). Four of the five seizures (including one prerandomization) occurred in patients with evidence of early brain injury on CT scans. Ten patients in the extended course group discontinued LEV early for agitation or sedation. Evaluation of functional/cognitive outcomes are pending.

\section{Conclusions:}

This is the first RCT studying seizure prophylaxis in SAH. While it showed a trend to more seizures in those receiving only 3 -days of LEV, it was underpowered to determine significance. A larger RCT appears warranted to evaluate the optimal regimen. In the interim, prophylaxis may be considered in those with evidence of early brain injury on CT scans. 


\title{
Poster 167
}

\section{Benzodiazepine Use For Status Epilepticus In An Academic Medical Center Emergency Department}

\author{
James T. Braun ${ }^{1}$, Stacy S. Revelle ${ }^{1}$, Elizabeth E. Gau', Laurie L. Byrne', Abhay A. Kumar ${ }^{3}$. \\ ${ }^{1}$ SSM Health Saint Louis University Hospital/Department of Pharmacy Services, Saint Louis, MO, USA, \\ ${ }^{2}$ Saint Louis University School of Medicine/Division of Emergency Medicine, Saint Louis, MO, USA, ${ }^{3}$ \\ Saint Louis University School of Medicine/Department of Neurology, Saint Louis, MO, USA.
}

\section{Introduction:}

Real-world studies regarding use of benzodiazepines in pre-hospital and emergency department (ED) settings for the treatment of Status Epilepticus (SE) are lacking. The purpose of this retrospective study was to analyze benzodiazepine usage patterns in SE by emergency medical services (EMS) and the ED of an inner-city hospital.

\section{Methods:}

Records of patients $\geq 18$ years old who presented with a diagnosis of idiopathic SE between June 2012 and September 2015 to EMS and hospital ED were reviewed. The associated outcomes of interest were endotracheal intubation, hospital admission, and seizure recurrence. Data was analyzed via descriptive statistics.

\section{Results:}

Of 44 patients analyzed, $43(97.7 \%)$ had a history of epilepsy. Benzodiazepine utilization varied; EMS preferred midazolam (69.2\%) while the ED used lorazepam most often (91.3\%). Benzodiazepine dosages used were lower than recommended; median dose of midazolam administered by EMS was only $5 \mathrm{mg}$ and median dose of lorazepam in the ED was $2 \mathrm{mg}$. Patients received $2.7 \pm 2.1$ benzodiazepine doses on average. Seizure activity was aborted with benzodiazepines alone in $22(50.0 \%)$ patients and recurred in $12(27.3 \%)$. Twenty-three (52.3\%) patients were intubated, all post- arrival. There was no observed correlation between number of benzodiazepine doses given and baseline characteristics, decision to intubate, or incidence of seizure recurrence. All 44 patients were admitted and $30(68.2 \%)$ were admitted to the ICU.

\section{Conclusions:}

We observed consistent underdosing of benzodiazepines used for the treatment of SE by both EMS and the ED. There was lower than expected achievement of seizure cessation and intubation rates were higher than reported in previous studies. Further investigation is needed to identify the barriers to optimal benzodiazepine selection and dosing for SE patients at our institution. 
Poster 168

\title{
Pharmacokinetics of SAGE-547 in patients with super refractory status epilepticus
}

\author{
Helen Colquhoun ${ }^{1}$, Ethan E. Hoffmann ${ }^{1}$, Shane S. Raines ${ }^{2}$, Jeff J. Wald ${ }^{3}$, Stephen S. Kanes ${ }^{1}$. \\ ${ }^{1}$ Sage Therapeutics, Inc., Cambridge, MA, USA, ${ }^{2} 2 b$ Analytics, Wallingford, PA, USA, ${ }^{3}$ PPharmetra, LLC, \\ Cary, NC, USA.
}

\section{Introduction:}

Super-refractory status epilepticus (SRSE) refers to a condition of persistent seizures that have failed treatment with first-, second- and third-line treatments. SAGE-547, a proprietary formulation of the endogenous neuroactive steroid allopregnanolone (a potent positive allosteric modulator of synaptic and extrasynaptic GABAA receptors in animal models), is being developed for the treatment of patients with SRSE who have not responded to standard treatment regimens. 547-SSE-201 was an open-label, phase $1 / 2$ study designed to evaluate the safety, pharmacokinetics (PK), and efficacy of SAGE-547 in patients with SRSE. The present analysis explores the PK properties of SAGE-547 over the course of the trial.

\section{Methods:}

SAGE-547was administered as a 5-day continuous intravenous infusion to 25 patients with SRSE and receiving third line agents (TLAs) for seizure or burst suppression. Patients received either a standard dosing regimen $(n=19)$ or a high dose regimen $(n=6)$ and were subsequently weaned off TLAs and SAGE-547. The standard dose was chosen, based on a modeling approach, to achieve a mean plasma exposure roughly equivalent to the highest endogenous concentrations measured in the third trimester of pregnancy $(\sim 157 \mathrm{nM})$. Since women tolerate this endogenous level without apparent adverse effects, 150 $\mathrm{nM}(47 \mathrm{ng} / \mathrm{mL})$ was selected as the target exposure for this study.

\section{Results:}

Mean (SD) steady-state concentration observed for the standard dose regimen was $66.2(21.93) \mathrm{ng} / \mathrm{mL}$, and for the high dose regimen was $113.6(42.08) \mathrm{ng} / \mathrm{mL}$. Steady-state concentrations were approximately dose proportional between the standard and high doses, indicating that clearance was dose-independent (mean (SD) of $1.36(0.56) \mathrm{L} / \mathrm{h} / \mathrm{kg}$ ). There was insufficient sampling following the end of infusion to allow determination of half-life or volume of distribution.

\section{Conclusions:}

In this study of patients with SRSE, SAGE-547 clearance was not dose-dependent and plasma concentrations were in line with target exposures. 


\section{Poster 169}

\section{Early withdrawal of non-sedating antiepileptic drugs after successful termination of nonconvulsive seizures and nonconvulsive status epilepticus}

Jennifer A. Creed, Christa C. Swisher.

Duke University Medical Center / Department of Neurology, Durham, NC, USA.

${ }^{* * * * P e r m i s s i o n ~ w a s ~ n o t ~ g r a n t e d ~ t o ~ p r i n t ~ t h i s ~ a b s t r a c t * * * *}$ 
Poster 170

\title{
Effect of Continuous Electroencephalography Coupled with Aggressive Seizure Suppression on Outcomes after Cardiac Arrest
}

\author{
John Faro ${ }^{1}$, Patrick P. Coppler ${ }^{2}$, Cameron C. Dezfulian ${ }^{2}$, Maria M. Baldwin ${ }^{3}$, Jon J. Rittenberger ${ }^{1}$, Clifton \\ C. Callaway ${ }^{1}$, Jonathan J. Elmer ${ }^{4}$.
}

${ }^{1}$ Department of Emergency Medicine, University of Pittsburgh, Pittsburgh, PA, USA, ${ }^{2}$ Department of Critical Care Medicine, University of Pittsburgh, Pittsburgh, PA, USA, ${ }^{3}$ Department of Neurology, University of Pittsburgh, Pittsburgh, PA, USA, ${ }^{4}$ Departments of Emerg Medicine and Crit Care Medicine, University of Pittsburgh, Pittsburgh, PA, USA.

\section{Introduction:}

Seizures after resuscitation from cardiac arrest predict worse outcomes, but there is no evidence that treating seizures improves outcomes. We leveraged existing practice variation to compare the effectiveness of aggressive electroencephalography (EEG) and antiepileptic drug (AED) use to infrequent spot EEG and AED use.

\section{Methods:}

We performed a retrospective cohort study including comatose post-arrest patients at two academic centers from 2011-2014. The same critical care group staffs both, but Center 1 uses continuous EEG (cEEG) monitoring and aggressively treats malignant EEG patterns while Center 2 uses infrequent spot EEGs and rarely treats with AEDs. We classified each patient's daily EEGs from admission until death, awakening or day 5 as "malignant" (any status epilepticus, polyspikes or periodic discharges), "not malignant," or "not performed." We abstracted covariates and outcomes from our prospective registry, including age, illness severity (Pittsburgh Cardiac Arrest Category), shockable rhythm, arrest location, survival to discharge and functionally favorable survival. We used multi-level mixed-effects logistic models to test for an association of Center with outcomes after adjusting for EEG and clinical covariates.

\section{Results:}

We included 824 subjects (Center 1:513, Center 2:311). Center 1 subjects were younger, arrested more often out-of-hospital and had higher illness severity (all $P<0.01)$. Overall, $366(71 \%)$ Center 1 subjects were EEG-monitored (median 2 days (IQR 1-4d)), 196 (38\%) had a malignant pattern observed and 227 $(44 \%)$ received AEDs (median 2/patient). In comparison, 155 (50\%) Center 2 subjects were monitored a median of 1d (IQR 1-1d), 62 (20\%) had malignant patterns observed (less frequent myoclonic status epilepticus than Center 1), and 89 (29\%) received AEDs (median 1/patient) ( $P<0.01$ for all betweencenter comparisons). In multilevel modeling, there was no significant Center effect on outcomes.

\section{Conclusions:}

After cardiac arrest, treatment at a center using aggressive cEEG monitoring and AED treatment is not associated with better outcomes at discharge. 


\section{Poster 171}

\section{Phenytoin Dosing Adjustment for Obesity may not be Necessary}

Lisa R. Yanase ${ }^{1}$, Michelle M. Chang ${ }^{2}$, Jillian J. Myers ${ }^{2}$, Lindsay L. Lucas ${ }^{1}$, Elizabeth E. Baraban ${ }^{1}$.

${ }^{1}$ Providence Brain and Spine Institute, Portland, OR, USA, ${ }^{2}$ Providence Department of Pharmacy, Portland, OR, USA.

\section{Introduction:}

Effective loading with weight-based dosing of phenytoin for therapeutic levels is necessary in several emergent settings. Practices for dosing obese patients, those $>1.3 \times$ Ideal Body Weight (IBW) vary, including using Total Body Weight (TBW), Adjusted Body Weight (AdjBW; correction factor 0.4), and the Abernathy formula (correction factor 1.33). Our objective was to determine whether dose adjustments were necessary for obese patients.

\section{Methods:}

Charts were reviewed retrospectively from two tertiary medical centers from September 2014 to August 2015. We included all admitted patients older than 18 years of age, initiated on IV fosphenytoin for any reason, with therapeutic post-load level (total phenytoin of $10-20 \mathrm{mcg} / \mathrm{mL}$, or free phenytoin $1-2 \mathrm{mcg} / \mathrm{mL}$ in pre-specified patients) at 6 hours following dose. Patients currently taking phenytoin were excluded. Mean weight-based loading doses were compared for obese and non-obese patients who achieved post load levels in the therapeutic and high therapeutic range (total level 15-20, free 1.5-2.0), using Welch's two-sample t-tests.

\section{Results:}

A total of 56 patients, 39 non-obese and 17 obese, met inclusion criteria, including 20 non-obese and 7 obese patients who achieved high therapeutic levels, desired for ongoing status epilepticus. The mean loading dose, in $\mathrm{mg} / \mathrm{kg}$, that achieved therapeutic levels was $18.9(95 \% \mathrm{Cl}: 17.7,20.0)$ for non-obese and $16.4(95 \% \mathrm{Cl}: 14.5,18.2)$ for obese patients $(\mathrm{t} 29=2.4, \mathrm{p}=0.02)$. Dose to achieve high therapeutic levels was $20.2(95 \% \mathrm{Cl}: 19.3,21.0)$ for non-obese and $18.1(95 \% \mathrm{Cl}: 15.9,20.3)$ for obese patients $(\mathrm{t} 9=2.1, \mathrm{p}=$ $0.06)$.

\section{Conclusions:}

Our results suggest that adjustment calculations of fosphenytoin loading dose for obese patients may not be necessary, thus can be simplified to $20 \mathrm{mg} / \mathrm{kg}$ TBW for all patients. This common dose is likely to get all patients into high therapeutic range, especially desirable in status epilepticus, while not harmful in other patients. 


\section{Poster 172}

\section{SAGE-547 for the treatment of super-refractory status epilepticus: response and relationship to underlying patient characteristics} Stephen J. Kanes ${ }^{1}$, Eric E. Rosenthal ${ }^{2}$, Henrikas H. Vaitkevicius ${ }^{3}$, Jan J. Claassen ${ }^{4}$, Mark M. Wainwright ${ }^{5}$,
Ethan E. Hoffmann ${ }^{1}$, Mollie M. Baird ${ }^{1}$, Mike M. Quirk ${ }^{1}$, Helen H. Colquhoun ${ }^{1}$.

${ }^{1}$ Sage Therapeutics, Inc., Cambridge, MA, USA, ${ }^{2}$ Massachusetts General Hospital, Boston, MA, USA, ${ }^{3}$ Brigham and Women's Hospital, Boston, MA, USA, ${ }^{4}$ Columbia University Medical Center, New York, NY, USA, ${ }^{5}$ Northwestern University Feinberg School of Medicine, Chicago, IL, USA.

\section{Introduction:}

SAGE-547 is a proprietary formulation of allopregnanolone. SAGE-547 was studied in an open-label phase $1 / 2$ clinical study of patients with super-refractory status epilepticus (SRSE). The primary objectives of the study were to evaluate the safety, tolerability, and pharmacokinetics (PK) of SAGE-547. The key secondary objective was to assess the efficacy of SAGE-547 in resolving SRSE in these patients. To further understand the specific patient and treatment-related parameters which may affect outcomes in this study, we performed a post-hoc analysis on the completed data set.

\section{Methods:}

In this open-label, single-arm study, patients $\geq 2$ years old with SRSE, on a continuous intravenous infusion of $\geq 1$ third-line agent (TLA; propofol, midazolam, pentobarbital, ketamine), underwent 5 days of treatment with SAGE-547. Key efficacy outcome measures were: 1) successful wean off of TLA(s) after hour 48; and 2) subsequent successful taper off SAGE-547 after hour 96, without recurrence in the 24hour period following treatment.

\section{Results:}

A total of 25 patients received open-label treatment with SAGE-547. Overall, 17/22 evaluable patients (77\%) were successfully weaned off TLA(s) and 16/22 (73\%) were successfully weaned off TLA(s) and SAGE-547. Response rate appeared consistent across varying patient demographics (gender, age, ethnicity) and baseline treatment regimens. Overall, $64 \%$ of patients experienced at least 1 serious adverse event (SAE) and 6 patients died during the trial. No SAEs and no deaths were attributed by the Safety Committee to SAGE-547 administration.

\section{Conclusions:}

There was little evidence of a relationship between response rate and patient demographics (gender, age, ethnicity). In a critically ill population with high baseline risk, this open-label phase $1 / 2$ clinical trial supports further investigation of SAGE-547 in SRSE, and can inform inclusion criteria for future trials. The clinical efficacy and safety of SAGE-547 in the treatment of SRSE is being evaluated further in an ongoing phase 3 , randomized, placebo-controlled trial. 


\title{
Poster 173
}

\section{Withdrawal of Care and Its Impact on Mortality in Status Epilepticus}

\author{
Jocelyn Y. Cheng. \\ Drexel University College of Medicine, Philadelphia, PA, USA.
}

\section{Introduction:}

In catastrophic neurologic injury, withdrawal of care (WOC) is often considered. While WOC is based on the perception of poor prognosis, the question of whether it creates a self-fulfilling prophecy has been raised. Though studied in traumatic brain injury and stroke, the effect of WOC on mortality in status epilepticus (SE) is unclear. The goal of this study was to describe the final cause of death in adults with $\mathrm{SE}$, and determine the impact and associated clinical characteristics of WOC on mortality rates.

\section{Methods:}

A single-center retrospective study at an urban academic medical center was conducted between $1 / 1 / 2005-10 / 31 / 2012$. Subjects aged $\geq 18$ years with SE were included. Data was collected on age;gender;Glasgow Coma Scale (GCS);Acute Physiology and Chronic Health Evaluation-II (APACHEII); history of epilepsy;etiology of SE;refractory SE (RSE);in-hospital mortality; and cause of death. Pearson's $X^{2}$ and t-tests were used as appropriate. Binary logistic regression analysis adjusted for covariates, and $p<0.05$ was considered significant.

\section{Results:}

Of 151 subjects, 68 male, mean age 59 years, there were $61(40.4 \%)$ in-hospital deaths, $45(74 \%)$ due to WOC. The remaining causes were cardiogenic $(\mathrm{N}=11,18 \%)$ and respiratory $(\mathrm{N}=2,3.2 \%)$, with sepsis, brain death and seizures individually comprising $1.6 \%(\mathrm{~N}=1$ each). Excluding WOC, in-hospital mortality fell to $15 \%(\mathrm{~N}=16 / 106)$. In the sub-group without cardiac arrest $(\mathrm{CA})$, in-hospital mortality was $25 \%(\mathrm{~N}=26 / 104)$, of which $14 \%(\mathrm{~N}=15)$ was due to WOC; mortality decreased to $12 \%(11 / 89)$ excluding WOC subjects. For the total cohort, there was no significant difference in baseline characteristics excepting metabolic seizure etiology $\left(N=6, x^{2}=8.97, p=0.003\right), C A\left(N=30, x^{2}=6.05, p=0.014\right)$, and RSE $\left(N=45, x^{2}=5.82, p=0.016\right)$, which were more common in WOC subjects. Metabolic etiology (OR:6.50, $p=0.005)$ and CA (OR:4.40, $p=0.018)$ remained significantly associated with WOC after univariate but not multivariate adjustment.

\section{Conclusions:}

Withdrawal of care is a major determinant of mortality in SE, and is associated with metabolic dysfunction and cardiac arrest. The impact of WOC on mortality rates in SE should be taken into consideration in clinical decision-making. 
Poster 174

\title{
Phase 1/2 open-label data suggest that heterogeneity of presentation and high burden of comorbid illness do not impact the activity of SAGE-547 in patients with super-refractory status epilepticus
}

\author{
Helen Colquhoun ${ }^{1}$, Mollie M. Baird ${ }^{1}$, Shane S. Raines ${ }^{2}$, Jeffrey J. Jonas ${ }^{1}$, Stephen S. Kanes ${ }^{1}$. \\ ${ }^{1}$ Sage Therapeutics, Inc., Cambridge, MA, USA, ${ }^{2} 2 b$ Analytics, Wallingford, PA, USA.
}

\section{Introduction:}

An open-label, phase 1/2 study (547-SSE-201) evaluated the safety and efficacy of SAGE-547, a proprietary formulation of allopregnanolone, in patients with super-refractory status epilepticus (SRSE). Entry criteria were designed to maximize patient treatment opportunities. The objective of this post-hoc analysis was to demonstrate SAGE-547 activity despite heterogeneity of SRSE causes and high comorbidity burden.

\section{Methods:}

Patients aged $\geq 2$ years with SRSE, on a continuous intravenous infusion of $\geq 1$ third-line agent (TLA; propofol, midazolam, pentobarbital, ketamine) for seizure or burst suppression were eligible if breakthrough seizures occurred during a wean attempt from, or during maintenance, of the TLA. Patients with anoxic brain injury or very short life expectancy were excluded.

\section{Results:}

Overall, 9/25 patients were female. Mean age was 48 years (range 10-76 years) and mean status epilepticus duration was 9.2 days (range 3-20 days). Probable SRSE causes included infection, hemorrhage, worsening seizures, brain tumors, toxic ingestion, and unknown causes. All patients had $\geq 5$ significant comorbid conditions, and $23 / 25$ were rated to be among the most extremely ill patients, using CGI-S score. All patients required 1-2 TLAs and 1-5 AEDs at baseline. Up to 8 weans from TLAs were attempted prior to enrollment. Despite SRSE severity and comorbidity burden, 17/22 evaluable patients (77\%) were successfully weaned off TLA(s) and 16/22 (73\%) were successfully weaned off TLA(s) and SAGE-547 at the end of 5 days; $11 / 22$ patients $(50 \%)$ had a final Glasgow Coma Scale total score $\geq 13$. Six patients $(24 \%)$ died from underlying SRSE cause or associated comorbid conditions. No SAEs were attributed by the Safety Committee to SAGE-547. Mean numbers of baseline wean attempts, comorbid conditions, and SRSE episode duration were comparable between responders and non-responders.

\section{Conclusions:}

It is feasible to prospectively study very sick patients with SRSE, and to demonstrate potential drug activity despite the heterogeneity of cause of SRSE and comorbidity burden. 


\title{
Poster 175
}

\section{Ictal-interictal Continuum (IICA) EEG patterns in Subarachnoid Hemorrhage with Vasospasm}

\author{
Michael S. Mendoza ${ }^{1}$, Owen O. Samuels ${ }^{1}$, Prem P. Kandiah ${ }^{1}$, Kathleen K. Martin ${ }^{1}$, Jan J. Vlachy ${ }^{2}$, Turgay \\ T. Ayer ${ }^{3}$, Andres A. Rodriguez-Ruiz ${ }^{4}$.
}

${ }^{1}$ Emory University, SOM/ Department of Neurosurgery, Atlanta, GA, USA, ${ }^{2}$ Georgia Institute of Technology/ Industrial and Systems Engineering, Atlanta, GA, USA, ${ }^{3}$ Georgia Institute of Technology/ Institute and Systems Engineering, Atlanta, GA, USA, ${ }^{4}$ Emory University, SOM/ Department of Neurology, Atlanta, GA, USA.

\section{Introduction:}

Delayed neurologic deterioration (DND) from vasospasm is associated with poor outcome after subarachnoid hemorrhage. Continuous EEG (CEEG) monitoring has lead to detection of EEG patterns of uncertain clinical significance - Ictal-interictal continuum (IICA). These have been associated with acute brain injury but variably predict outcome. We describe the types and prevalence of IICA EEG patterns in patients who develop angiographic vasospasm and discharge outcomes

\section{Methods:}

Retrospective study of adult patients with non-traumatic subarachnoid hemorrhage admitted at Emory University Hospital Neuro-ICU from January 2013-February 2016 who underwent CEEG. CEEG were recorded using 10-20 electrode placement and interpretation of the IICA EEG patterns using the American Clinical Neurophysiology Society research terminology.

\section{Results:}

In $131 \mathrm{SAH}$ patients, 95(73\%) were female and hypertensive 88 (67\%). Majority had poor grade SAH (40\% HH Grade 3, 24\% grade 4 and $17 \%$ grade 5). Moderate to severe angiographic vasospasm were detected in 74 (56\%) patients 5.7 days after admission. CEEG was initiated 4.17 days after admission. Periodic discharges (PDs) occurred in 42(32\%) patients, 14(33.3\%) of which were generalized and 19 $(45.2 \%)$ lateralized. Rhythmic Delta Activity (RDA) occurred in $33(25.10 \%)$ with $26(78.7 \%)$ generalized. Stimulus induced Rhythmic Discharges (SIRPIDS) were seen in $9(6.8 \%)$ and electrographic seizures in $5(3.80 \%)$ patients. Vasospasm was common in patients with any IICA patterns ( $77.5 \%$ vs. $22.4 \% p=0.36)$, PDs $(77.5 \%$ vs. $22.4 \% \mathrm{p}=0.56)$ and RDAs $(84.3 \%$ vs. $15.6 \% \mathrm{p}=0.12)$. RDAs were common in patients with discharge MRS $0-4(59.3 \%$ vs. $40.7 \% \mathrm{p}=0.12)$ and PDs were equally seen across all outcomes $(50 \%$ vs. $50 \% \mathrm{p}=0.56)$. The observed trends were not statistically significant.

\section{Conclusions:}

IICAs such as PDs and RDAs were common in patients who developed vasospasm but seen equally across all outcomes suggesting that such patterns are markers of acute brain injury. A prospective study with a larger sample size is needed to support these findings. 
Poster 176

\title{
Non-Convulsive Seizures (NCS)/Non-Convulsive Status Epilepticus (NCSE) in Neuro-ICU: Clinical Predictors and Prognostication
}

\author{
Marta Melis ${ }^{1}$, Moshe M. Mizrahi ${ }^{2}$, Lara L. Marcuse ${ }^{3}$, Madeline M. Fields ${ }^{3}$, Jiyeoun J. Yoo ${ }^{3}$, Neha N. \\ Dangayach $^{2}$, Errol E. Gordon ${ }^{2}$, Stephan S. Mayer'. \\ ${ }^{1}$ University of Cagliari/Neurology, Cagliari, Italy, ${ }^{2}$ Mount Sinai Health System/Neurosurgery and \\ Neurology, New York, NY, USA, ${ }^{3}$ Mount Sinai Health System/Neurology, New York, NY, USA.
}

\section{Introduction:}

NCS and (NCSE) have been reported in $8-25 \%$ of critically ill patients. Whether patients with NCSE should be treated as aggressively as patients with convulsive status remains controversial. This study seeks to describe the characteristics of NCS/NCSE, focusing on its correlation with patients' outcome and possible predictors.

\section{Methods:}

In this retrospective study $n=261$ patients underwent $c E E G$ at Mount Sinai Neurological and Neurosurgical ICU (NSICU). cEEG data according to ACNS guidelines was collected. Outcome was evaluated by mortality, Glasgow outcome scale (GOS), Glasgow Coma scale (GCS). We compared 29 patients in NCSE/NCS Group (NG) with Control Group (CG) of 148 patients.

\section{Results:}

Seizures were detected in $41 \%$ of subjects and $11 \%$ experienced NCS/NCSE. Significant risk factors included diagnosis of epilepsy, both as a reason for hospitalization, $27 \%(8 / 29)$ of NG and in $1 \%(2 / 148)$ of CG ( $p=0.000$ OR $27.8 \mathrm{Cl} 5.53-139)$, and as a previous diagnosis, $17 \%(5 / 29)$ of NG and in $4 \%(6 / 148)$ of CG ( $p=0.019$, OR 4.93, CI 1.39-17.44). Considering clinical predictors, only gaze deviation and subtle facial movements were significant $(p=0.003$ OR 4.7, Cl 1.7-12.3, $p=0.000$, OR $38 \mathrm{Cl} 7-188)$. Mortality and mean hospitalization length were not different. Outcome was significantly different in NSICU with mean GCS being 8 in NG and 11 in CG ( $\mathrm{S}$ for $p=0.004$ ), but was not significantly different at discharge, as mean GOS was 2 in NG and 2.4 in $C G(p=0.076)$.

\section{Conclusions:}

Our findings show that patients' history of epilpesy, specific clinical signs like gaze deviation and facial twitching might help in identifying subjects at high risk of NCSE/NCS in the NSICU. The implications for treatment of NCSE/NCS remain controversial and more prospective studies are needed to understand prognostication in these patients. 


\title{
Poster 177
}

\section{A Practical Approach to Ketogenic Diet in the Pediatric Intensive Care Unit for Super Refractory Status Epilepticus}

\author{
Raquel Farias-Moeller, Archana A. Pasupuleti, Luca L. Bartolini, Amy A. Kao, Brittany B. Cines, Jessica J. \\ Carpenter.
}

Children's National Health System, Washington, DC, USA.

\section{Introduction:}

Super refractory status epilepticus (SRSE) ensues when there is no improvement of seizure control in response to anesthetic therapy or seizure recurrence after reduction of anesthetic agents. There is no consensus on standard of care for SRSE. Ketogenic diet (KD) has reported success but technical challenges exist including inability to feed patients, concomitant steroid use, persistent acidotic states and lack of dieticians with experience. The optimal protocol for KD is yet to be determined. We describe our step-by-step approach to initiation and continuation of KD in the PICU.

\section{Methods:}

Patients with SRSE who had KD initiation in the PICU were identified from a prospective Neurocritical Care database with IRB approval. Data from the hospital course was supplemented by review of the electronic medical record. Descriptive analysis was performed. Neurointensivists used our step-by-step guideline to start patients on KD.

\section{Results:}

Nine children with SRSE who had KD initiated in the PICU were identified. The mean age was 5.4 years (SD 2.24). Median number of days to start KD from detection of seizures was 13 [IQR 10-16]. Mean time to achieve ketosis was 4.2 days (SD 3.4). Five of the nine children remained on the KD for 3 months or longer. The median number of AEDs trialed before KD was started was 4 [IQR 3-4] and the median number of continuous infusions was 2 [IQR 2-3]. After initiation of KD most patients were weaned off continuous infusions by 1 week after initiation. Outcomes were variable.

\section{Conclusions:}

We demonstrated the feasibility of a practical approach to initiation of KD in the PICU for children with SRSE. These children were successfully weaned off continuous anesthetic infusions. Larger studies, both in children and adults, are needed to determine the effectiveness, safety and tolerability of KD in the management of SRSE as well as its ease of implementation. 
Poster 178

\title{
External Validation of the Epidemiology-Based Mortality Score in Status Epilepticus Score in an American Population
}

\author{
Nirupama Yechoor ${ }^{1}$, Amir A. Adeli ${ }^{2}$, Shaheryar S. Hafeez ${ }^{2}$. \\ ${ }^{1}$ Washington University School of Medicine / Neurology, St. Louis, MO, USA, ${ }^{2}$ The Ohio State University \\ Wexner Medical Center, Columbus, OH, USA.
}

\section{Introduction:}

Although overall mortality of status epilepticus is high, baseline patient characteristics and co-morbidities may help to predict outcomes and shape treatment decisions. Two previously published scores exist to predict outcomes: the Status Epilepticus Severity Score (STESS) and the Epidemiology-based Mortality Score in Status Epilepticus (EMSE). However, a comparison of the two scores has not previously been completed in an American population. We hypothesize that both scores will adequately predict the primary outcome of in-hospital death.

\section{Methods:}

We performed a retrospective analysis of all cases of status epilepticus admitted to the Neuro-Critical Care Unit (NCCU) at the Ohio State University Wexner Medical Center from 6/1/2014 - 8/31/2015. We collected data on age, comorbidities, EEG findings, and seizure history. The primary outcome was inhospital death. A sensitivity and specificity analysis was completed, in addition to a student's t-test for a comparison of the two scores.

\section{Results:}

Forty-six patients were admitted to the NCCU for management of status epilepticus during June 2014 and January 2016, 13 of which experienced in-hospital death. The median age of the sample was 60, with approximately half of the sample $(52.6 \%)$ having 3 or more comorbidities. The two most common etiologies were cryptogenic $(n=9)$ and acute cerebrovascular events $(n=7)$. While the sensitivity of both EMSE and STESS were very high (100\% and $90 \%$ respectively), the specificities were very low $(28.6 \%$ and $42.9 \%$ respectively). A student's t-test between those who experienced in-hospital death and those who did not was only significant for EMSE at the $p<0.1$ level $(p=0.055)$.

\section{Conclusions:}

The specificity of EMSE and STESS for our external validation did not correlate with previous studies; however, both tools are sensitive. The EMSE and STESS may be useful to predict outcomes of status epilepticus in populations with few comorbid conditions, but are less helpful when patients have multiple medical problems. 


\title{
Poster 179
}

\section{Lost in translation: Survey of American Clinical Neurophysiology Society (ACNS) standardized critical care electroencephalography (EEG) terminology}

\author{
Chalita C. Atallah ${ }^{1}$, Neeraj N. Badjatia ${ }^{2}$, Jennifer J. Pritchard ${ }^{1}$. \\ ${ }^{1}$ University of Maryland School of Medicine, Department of Neurology - Epilepsy Division, Baltimore, MD , \\ USA, ${ }^{2}$ University of Maryland SOM - Program in Trauma, Section of Neurocritical Care, Baltimore, MD, \\ USA.
}

\section{Introduction:}

In 2012, the ACNS published critical care EEG terminology in an effort to improve clinical research and management of patients requiring continuous EEG (cEEG) monitoring. We sought to understand the familiarity of providers in our Neurocritical care (NCC) program with this terminology two years after implementation at our institution.

\section{Methods:}

We administered a 21 question web-based survey to NCC fellows, advanced practice providers (APPs), and attendings. Questions assessed basic EEG knowledge, understanding of ACNS terminology, and clinical EEG application.

\section{Results:}

There were 5 attending physicians, 4 NCC fellows, and 13 APPs. Attending physicians and APPs had a median of 4 (range: 3,13 ) and 2 years (range: $0.25,15$ ) experience since most recent post graduate training, respectively. All data is reported for APPs and physicians respectively as percentage correct. Basic knowledge of EEG frequencies and reactivity $15.4 \%$ and $77.8 \% ; 7.7 \%$ and $55.6 \%$ respectively; ACNS electrographic seizure definition $84.6 \%$ and $88.9 \%$. Main terms: generalized $38.5 \%$ and $66.7 \%$; lateralized $7.7 \%$ and $44.4 \%$; bilateral independent $61.5 \%$ and $77.8 \%$; multifocal $23.1 \%$ and $66.7 \%$. Modifiers: +F $46.2 \%$ and $88.9 \%$; $+\mathrm{R} 76.9 \%$ and $100 \%$; $+\mathrm{S} 53.9 \%$ and $100 \%$. Attenuation/suppression descriptors: continuous $53.9 \%$ and $66.7 \%$; near continuous $15.4 \%$ and $77.8 \%$; discontinuous $15.4 \%$ and $77.8 \%$; burst suppression $30.8 \%$ and $55.6 \%$; suppression $23.1 \%$ and $55.6 \%$. Prevalence descriptors: continuous 53.8 and $100 \%$; abundant $53.8 \%$ and $88.9 \%$; frequent/occasional/rare $61.5 \%$ and $88.9 \%$. Duration descriptors: very long/long/intermediate $76.9 \%$ and $100 \%$; brief/very brief $69.2 \%$ and $88.9 \%$. The highest rated component of the cEEG report that influenced patient management was "conversation with epilepsy fellow/attending," selected by $92.3 \%$ of APPs and $100 \%$ of physicians.

\section{Conclusions:}

Responses from this survey demonstrate a wide spectrum of knowledge in understanding the terminology set forth by the ACNS and highlight the importance of communication between NCC providers and epileptologists as well as areas of potential education for providers of all training levels. 
Poster 180

Intravenous Carbamazepine: A New Formulation of a Familiar Drug

Pavel Klein ${ }^{1}$, Dwain D. Tolbert ${ }^{2}$, Jouko J. Isojarvi ${ }^{2}$.

${ }^{1}$ Mid-Atlantic Epilepsy and Sleep Center, Bethesda, MD, USA, ${ }^{2}$ Lundbeck LLC, Deerfield, IL, USA.

\section{Introduction:}

Carbamazepine (CBZ), an oral antiepileptic drug (AED), is a potent inducer of cytochrome P450 (CYP) isozymes. Patients receiving CBZ who are unable to take drugs orally must switch to an IV AED option (eg, phenytoin, fosphenytoin, phenobarbital, valproic acid, levetiracetam, or lacosamide) to reduce the risk of breakthrough seizures; however, patients may experience potential changes in concomitant-drug metabolism (reduced efficacy or toxicity). An IV CBZ formulation has been developed; study OV-1015 (NCT01079351) evaluated bioequivalence and 13181A (NCT01128959) evaluated tolerability.

\section{Methods:}

Both studies were similarly designed. Eligible adult patients received a stable oral CBZ regimen (4002,000 mg/day) for 28 days (13181A: 27 days) prior to switching to IV CBZ infused at $70 \%$ of the oral total daily dosage (divided doses q6h) during the confinement period (OV-1015: 15- or 30-min infusions q6h for 7 days, patients in the 15-min group were eligible to receive four 2- to 5-min infusions on Day 8; 13181A: 15-min infusions q6h for 4 days, then one 5-min infusion on Day 5). Oral CBZ was resumed for 30 days (13181A: 28 days). Bioequivalence of IV to oral CBZ was evaluated in OV-1015; tolerability data were pooled.

\section{Results:}

In OV-1015, 30-min IV CBZ infusions were within the 80\%-125\% bioequivalence range vs oral CBZ; 15 min infusions exceeded the upper limit for maximum plasma concentration. In both trials, 203 patients switched to IV CBZ (30-min: $n=43 ; 15$-min: $n=160)$. The only AE term reported in $\geq 10 \%$ of patients during infusion was dizziness (19\%); infusion-site reactions (12\%) were the only new AEs experienced by patients vs oral CBZ. Seizure control was maintained during the switch.

\section{Conclusions:}

To avoid potential drug toxicity reactions, it is beneficial to maintain patients on CBZ. IV CBZ administered as multiple 30-min infusions was bioequivalent to oral CBZ. IV CBZ was well tolerated. 


\title{
Poster 181
}

\section{PHARMACOKINETICS AND HEALTH ECONOMICS OF INTRAVENTRICULAR SUSTAINED RELEASE NIMODIPINE (EG-1962) FOR SUBARACHNOID HEMORRHAGE}

\author{
Daniel Hänggi ${ }^{1}$, R. Loch R. Macdonald ${ }^{2}$, Nima N. Etminan ${ }^{3}$, Hans Jakob H. Steiger ${ }^{4}$, Stephan S. Mayer ${ }^{5}$, \\ Francois F. Aldrich ${ }^{6}$, Michael M. Diringer ${ }^{7}$, Brian B. Hoh ${ }^{8}, \mathrm{~J} J$. Mocco $^{5}$, Poul P. Strange ${ }^{9}$, Herbert H. \\ Faleck $^{10}$, Michael M. Miller'.
}

\footnotetext{
${ }^{1}$ Department of Neurosurgery, Universitätsmedizin Mannheim, Universität Heidelberg, Mannheim, Germany, ${ }^{2}$ Department of Neurosurgery, St. Michael's Hospital, Toronto, Canada, ${ }^{3}$ University Hospital Mannheim, University of Heidelberg Mannheim, Dusseldorf, Germany, ${ }^{4}$ Heinrich-Heine-University, Dusseldorf, Germany, ${ }^{5}$ Mount Sinai Hospital, New York, USA, ${ }^{6}$ University of Maryland Medical Center, Baltimore, USA, ${ }^{7}$ Washington University, St. Louis, USA, ${ }^{8}$ University of Florida, Gainesville, USA, ${ }^{9}$ Integrated Medical Development, Princeton Junction, USA, ${ }^{10}$ Edge Therapeutics, Inc., Berkeley Heights , USA.
}

\section{Introduction:}

Treatments for aneurysmal subarachnoid hemorrhage (aSAH) remain inadequate. EG-1962 is a sustained release formulation of nimodipine for intraventricular delivery in order to avoid dose-limiting hypotension common with systemic administration. We report the pharmacokinetics and health economics obtained from a phase 1/2a multicenter, controlled, randomized, open-label, dose escalation study of a single intraventricular dose of EG-1962 that was designed to determine the maximum tolerated dose (MTD) and assess safety, tolerability, pharmacokinetics and efficacy at 90 days on the extended Glasgow outcome scale (www.clinicaltrials.gov Identifier: NCT01893190).

\section{Methods:}

Subjects with aSAH repaired by clipping or coiling were randomized within 60 hours of aSAH to EG-1962 or oral nimodipine if they were World Federation of Neurological Surgeons grade 2 to 4 and had a ventricular catheter. Cohorts of 12 subjects received 100, 200, 400, 600, 800 or 1200 mg EG-1962 (9 per cohort) or oral nimodipine ( 3 per cohort). Plasma nimodipine concentrations were sustained for 21 days.

\section{Results:}

The maximum concentration, steady state concentration and area under the curve for the first 14 days increased with increasing dose of EG-1962 (Figure). There was no difference in pharmacokinetics between males and females. Plasma nimodipine concentrations following EG-1962 administration did not exceed plasma concentrations of oral nimodipine $60 \mathrm{mg}$ every 4 hours at steady state. Cerebrospinal fluid nimodipine concentrations with EG-1962 were orders of magnitude higher than in plasma or with oral nimodipine. Subjects treated with EG-1962 $(n=45)$ had a median intensive care stay 3.5 days less and hospital length of stay 2.5 days less than subjects treated with enteral nimodipine ( $n=18$, Table). Intraventricular EG-1962 produced sustained, dose-dependent nimodipine plasma concentrations and shortened intensive care and hospital length of stay.

\section{Conclusions:}

These findings, along with the already reported favorable safety profile, lack of systemic hypotension and improved clinical outcome support conduct of a pivotal phase 3 study of EG-1962. 


\title{
Poster 182
}

\section{Total Radiation Exposure during Initial Hospitalization in Patients with Aneurysmal Subarachnoid Hemorrhage: A Retrospective Observational Study}

\author{
Robin A. Bush', Brian B. Fischer ${ }^{1}$, Justin J. Fraser ${ }^{2}$, Abdulnasser A. Alhajeeri ${ }^{3}$, Kevin K. Hatton ${ }^{1}$, Arnold \\ A. Stromberg ${ }^{4}$. \\ ${ }^{1}$ University of Kentucky/Anesthesiology, Lexington, KY, USA, ${ }^{2}$ University of Kentucky/Neurosurgery, \\ Lexington, $K Y, U S A,{ }^{3}$ University of Kentucky/Radiology, Lexington, $K Y, U S A,{ }^{4}$ University of \\ Kentucky/Statistics, Lexington, KY, USA.
}

\section{Introduction:}

Increasing exposure to ionizing radiation for medical diagnostics and treatment has raised questions about possible long term effects. This study describes the effective dose of ionizing radiation exposure in patients with aneurysmal subarachnoid hemorrhage (aSAH).

\section{Methods:}

Thirty-five aSAH patients admitted to a university hospital between Jan 1, 2015 and December 31, 2015, greater than 18 years old, and discharged alive were included. The primary objective was to calculate the mean total effective dose of ionizing radiation (TEDIR) in aSAH patients. Secondarily, the number of aSAH patients with a calculated TEDIR greater than the annual and cumulative maximal permissible radiation dose (MPRD) as described by the National Council of Radiation Protection and Measurements (NCRP) and the International Commission on Radiological Protection (IRCP) was determined. Factors associated with greater than maximal exposure limits were evaluated.

\section{Results:}

TEDIR exposure ranged from 5.7- 89.2 millisievert (mSv), with a mean (SD) of 32.1 (21.79) mSv. Seven patients (20\% of the cohort) exceeded NCRP limit $(50 \mathrm{mSv} / \mathrm{yr})$ and 20 patients $(57 \%)$ exceeded the ICRP limit $(20 \mathrm{mSv} / \mathrm{yr})$. Hunt/Hess $(\mathrm{HH})$ class 3 or $4(p<0.01)$, the presence of vasospasm $(p<0.01)$, external ventricular drain (EVD) $(p<0.01)$, or ventriculo-peritoneal Shunt (VPS) $(p<0.05)$ were statistically significant factors for increased TEDIR in aSAH. Demographic data, previous medical history, and location of aneurysm were not statistically significant. Univariate analysis representing the degree to which TEDIR increases for each of these factors revealed HH class ( 3 or 4$) 20.2(p<0.01)$, vasospasm 24.4 ( $p<0.01)$, EVD 22.3 ( $p<0.01)$, VPS 21.5 ( $p<0.05)$. In multivariate analysis representing the degree in which the TEDIR increases, only vasospasm $26.1(p<0.01)$ and EVD $14.4(p<0.01)$ were statistically significant factors.

\section{Conclusions:}

Following aSAH, patients with severe disease requiring vasospasm treatment and shunting are at particular risk for multiple radiation exposures. Studies aimed at limiting these exposures may be warranted. 


\section{Poster 183}

The Subarachnoid Hemorrhage Early Brain Injury Score (SEBIS) Predicts Outcome after SAH

Sungho Ahn ${ }^{1}$, Mubashir M. Pervez ${ }^{2}$, Jude P J. Savarraj ${ }^{2}$, Wesley W. Jones ${ }^{2}$, Jin J. Park ${ }^{2}$, Tiffany R T. Chang ${ }^{2}$, Kiwon K. Lee', Dong H. D. Kim², Arthur L A. Day ${ }^{2}$, H. Alex H. Choi'.

${ }^{1}$ Division of Neurocritical care, University of Texas Health Science Center at Houston, Houston, TX, USA,

${ }^{2}$ Division of Neurocritical care, University of Texas Health Science Center at Houston, Houston, USA.

****Permission was not granted to print this abstract ${ }^{\star * * *}$ 


\title{
Poster 184
}

\section{Biphasic Cerebral Blood Flow Response In Cerebral Hypoperfusion After Aneurysmal Subarachnoid Hemorrhage}

\author{
Halinder S. Mangat ${ }^{1}$, Nikita N. Dave ${ }^{2}$, Malik M. Fakhar ${ }^{3}$, Santosh S. Murthy ${ }^{3}$, Philip P. Stieg ${ }^{4}$, Pina P. \\ Sanelli. \\ ${ }^{1}$ Weill Cornell Medicine / Neurology \& Neurosurgery, New York, NY, USA, ${ }^{2}$ The College of New Jersey, \\ Ewing, NJ, USA, ${ }^{3}$ Weill Cornell Medicine / Neurology, New York, NY, USA, ${ }^{4}$ Weill Cornell Medicine / \\ Neurosurgery, New York, NY, USA, ${ }^{5}$ Northwell Health / Radiology, Manhasset, NY, USA.
}

\section{Introduction:}

Patients with aneurysmal subarachnoid hemorrhage (SAH) have high morbidity and mortality related to cerebral ischemia and infarction. In this study we explored the reversibility of reduction in cerebral blood flow (CBF) after SAH.

\section{Methods:}

We conducted a retrospective analysis using prospectively collected CT perfusion (CTP) data from SAH patients. Patients were grouped as good (Hunt Hess 1-2) and poor grades (HH 3-5). CTP data were compared at baseline (0-4 days after aneurysm rupture) and follow-up ( $>5$ days).

\section{Results:}

159 patients were included. 50\% (80/159) were classified as good grade and 50\% (79/159) as poor grade. $48 \%$ (77/159) patients underwent aneurysm clipping and $51 \%(81 / 159)$ underwent coiling. Global $\mathrm{CBF}$ at baseline was comparable between good and poor grade patients $(39.06 \pm 9.75$ vs. $38.16 \pm 11.00$ $\mathrm{mL} / 100 \mathrm{~g} / \mathrm{min}$., $\mathrm{p}=0.31)$. At follow-up there was an improvement from baseline in both groups $(42.27 \pm 9.08$ and $40.23 \pm 9.53$ respectively, $p=0.06$ ). However, in hypoperfused areas, $r C B F$ was significantly lower in poor grade patients compared to good grade $(24.71 \pm 9.06$ vs. $31.25 \pm 10.60, p=0.003)$ and significantly lower than global CBF in both groups $(p<0.001)$. At follow-up, only poor grade patients demonstrated an increase in rCBF $(29.02 \pm 9.55, p=0.01)$ while in good grade patients, $r C B F$ remains unchanged $(31.79 \pm 9.86, p=0.41)$. The absolute improvement in $\mathrm{rCBF}$ was significantly greater in poor grade patients $(+7.65 \pm 21.2)$, compared to good grade patients $(+0.06 \pm 17.76)(p=0.005)$. In addition, rCBF at follow-up in both groups was not statistically different in the two groups $(p=0.09)$ but significantly lower than global $\operatorname{CBF}(p<0.001)$.

\section{Conclusions:}

Regional hypoperfusion occurs after SAH. In good grade patients there is no recovery in rCBF while in poor grade patients there is partial recovery implying a biphasic response with a reversible and an irreversible reduction in $\mathrm{rCBF}$. This has not been previously described in the literature and may implicate two distinct mechanisms responsible for rCBF reduction after SAH. 


\title{
Poster 185
}

\section{Trends in External Ventricular Drain Use, Ventriculostomy-associated Infections, and Mortality in Aneurysmal Subarachnoid Hemorrhage: Data from the Nationwide Inpatient Sample}

\author{
Ranjita Raghavan ${ }^{1}$, Roy R. Poblete ${ }^{2}$, Ling L. Zheng ${ }^{3}$, Steven S. Cen ${ }^{2}$, Arun A. Amar ${ }^{4}$, William W. Mack ${ }^{4}$, \\ May M. Kim-Tenser.
}

${ }^{1}$ University of Southern California/Keck School of Medicine, Los Angeles, CA, USA, ${ }^{2}$ University of Southern California/Keck School of Medicine/Department of Neurology, Los Angeles, CA, USA, ${ }^{3}$ University of Southern California/Keck School of Medicine, Department of Neurology, Los Angeles, CA, USA, ${ }^{4}$ USC/Keck School of Medicine/Department of Neurosurgery, Los Angeles, CA, USA, ${ }^{5}$ USC/Keck School of Medicine/Department of Neurology, Los Angeles, CA, USA.

\section{Introduction:}

Aneurysmal subarachnoid hemorrhage (aSAH) is an important cause of morbidity and mortality, with patients susceptible to a variety of medical complications. External ventricular drains (EVDs) are commonly used for intracranial pressure monitoring and CSF drainage; however, this puts aSAH patients at risk for ventriculostomy-associated infections (VAls). Many preventative strategies have been proposed and implemented over the last 20 years. Using the Nationwide Inpatient Sample (NIS) database, this study examines trends in EVD usage, VAI rates, and mortality over a 12-year period.

\section{Methods:}

In this retrospective analysis, data from the NIS was obtained for the period of January 1, 2000 through December 31, 2011 using International Classification of Diseases, 9th revision (ICD-9) codes. Analysis was performed using SAS 9.4 surveymeans. Primary outcomes of interest were rates of EVD use, VAI and in-patient mortality, as well as hospital length of stay.

\section{Results:}

During the study period, there were 422,168 aSAH admissions, with EVD placement reported in $15.8 \%$ of cases. There was no change in either EVD use or rate of VAI (mean VAI rate of $5.91 \%$ over the 12 years). No change in hospital length of stay was observed. From January 1, 2000 to December 31, 2011, mortality has declined with a mean absolute risk reduction (ARR) of $0.83 \%$ per year.

\section{Conclusions:}

Despite a variety of VAI-preventative strategies advocated for and implemented, the national VAI rate in aSAH patients has not changed; however, mortality has declined by a mean ARR of $0.83 \%$ per year over the study period. This may reflect improved neurointensive care provided to this critically ill population. Stable VAl rates may reflect incomplete adoption of effective preventative strategies, or use of ineffective ones. Further studies are needed to determine how best to reduce VAls. To our knowledge, no study has previously examined these trends in aSAH. 


\section{Poster 186}

\section{Effect of steroids on outcomes after aneurysmal subarachnoid hemorrhage}

Mubashir Pervez ${ }^{1}$, Jude J. Savarraj ${ }^{2}$, Sungho S. Ahn ${ }^{2}$, Jin J. Park ${ }^{3}$, Wesley W. Jones ${ }^{2}$, Elena E. Espino ${ }^{2}$, Glenda G. Torres ${ }^{4}$, Sophie S. Samuel', Tiffany T. Chang', Huimahn H. Choi'.

\footnotetext{
${ }^{1}$ University of Texas Health Science Center at Houston/ Neurocritical care, Houston, TX, USA, ${ }^{2}$ University of Texas, Health science center at Houston, Houston, TX, USA, ${ }^{3}$ University of Texas, Health Science center at Hosuton, Houston, TX, USA, ${ }^{4}$ Unievrsity of Texas, Health science center at houston, Houston, TX, USA.
}

\section{Introduction:}

The purpose of this study was to determine the effect of routine use of dexamethasone on delayed cerebral ischemia and poor outcome (death and severe disability) after aneurysmal subarachnoid hemorrhage $(\mathrm{aSAH})$.

\section{Methods:}

This is a single center, observational cohort study comparing patients with aSAH admitted to a tertiary referral center from 2013 to 2015. A variation in practice patterns for the use of dexamethasone 4-6 mg every 6 hours after aSAH exists in our institution depending on neurosurgeon preference. Patients were followed prospectively for the occurrence of complications including delayed cerebral ischemia (DCl), incidence of infection, functional outcomes measured by the modified rankin scale (mRS) at discharge (bad outcome defined as a mRS of 4-6) and 3 months (bad outcome defined as mRS of 3-6).

\section{Results:}

Out of the 173 patients, $59(34 \%)$ patients received dexamethasone during the first 72 hours of admission. Significant factors associated with steroid use were females $(41 \% \vee 21 \% ; p=0.01)$ and aneurysm clipping verses coiling $(55 \% \vee 27 \% ; p<0.01)$. There was no difference in $\mathrm{HH}$, Fisher grade, incidence of infections, or incidence of $\mathrm{DCl}(34 \% \vee 23 \% ; \mathrm{p}=0.15)$. Steroid use was significantly associated with bad outcome at discharge $(72 \% \vee 50 \% ; p=0.01)$, but no difference at 3 months $(34 \% \vee 31 \% ; p=0.7)$. When examined separately for coiled and clipped patients similar trends were found in both subgroups. Steroid use was associated with a longer hospital length of stay (LOS). In multivariable regression analysis steroid use was significantly associated with worse outcome (OR 3.6; $p<0.01)$ when controlled for age, $\mathrm{HH}$ grade and type of surgical intervention.

\section{Conclusions:}

The study did not detect any benefit in the use of steroids in reducing the incidence of delayed cerebral ischemia in acute aneurysmal subarachnoid hemorrhage. However, steroid use was significantly associated with longer LOS, and worse functional outcome at discharge. 


\title{
Poster 187
}

\section{Antiplatelet therapy for the prevention of peri-coiling thromboembolism in high risk patients with ruptured intracranial aneurysms}

\author{
Nancy J. Edwards ${ }^{1}$, Wesley W. Jones ${ }^{2}$, Aditya A. Sanzgiri', Juan J. Corona ${ }^{2}$, Mark M. Dannenbaum ${ }^{2}$, \\ Peng P. Chen" ${ }^{2}$. \\ ${ }^{1}$ University of Texas Health Science Center at Houston; Neurosurgery and Neurology, Houston, TX, USA, \\ ${ }^{2}$ University of Texas Health Science Center at Houston; Neurosurgery, Houston, TX, USA.
}

\section{Introduction:}

Thromboembolic events (TEE) during or after coiling of intracranial aneurysms is the most frequent procedural complication, resulting in permanent neurologic disability in a subset of patients. In unruptured aneurysm patients, there is evidence supporting the use of periprocedural antiplatelet therapy to prevent TEE. Whether patients with ruptured aneurysms and subarachnoid hemorrhage should also be given peri-coiling antiplatelet therapy is less clear.

\section{Methods:}

We reviewed a prospective registry of 169 endovascularly treated aneurysm patients to delineate angiographic features associated with periprocedural TEE. We then performed a controlled before-andafter study in 79 patients with ruptured aneurysms at high risk for TEE (e.g. patients with at least 1 angiographic feature associated with TEE) to evaluate whether selective aspirin administration would reduce the rate of periprocedural thromboembolism without increasing major hemorrhagic complications.

\section{Results:}

6 angiographic features were identified as high risk for TEE: wide aneurysm neck, coil/loop protrusion, small parent artery diameter, an incorporated branch, intraprocedural thrombus formation, and parent vessel intracranial atherosclerosis. Aspirin administration to high risk patients significantly decreased the rate of periprocedural TEE, from $53.8 \%$ in the control group to $10.6 \%$ in the aspirin-treated group $(p=$ $0.001)$. TEE reduction in the aspirin-treated group continued to be statistically significant even when adjusted for age, sex, cardiovascular risk factors, and factors associated with TEE in other large studies with an adjusted OR of $0.16(95 \% \mathrm{Cl} 0.03-0.8)$. There were no major systemic hemorrhagic complications, and aspirin did not increase the risk of aneurysm re-bleed, symptomatic intracranial hemorrhage, or major external ventricular drain (EVD)-associated hemorrhage $(p=0.3)$.

\section{Conclusions:}

Our findings suggest that for ruptured aneurysm patients with high risk features, antiplatelet therapy can significantly reduce the rate of peri-coiling TEE without increasing major systemic or intracranial hemorrhages. 


\section{Poster 188}

Prediction of thrombotic complications and outcome in subarachnoid hemorrhage patients using thromboelastography

Jin Park', Sung ho S. Ahn'², Jude J. Savarraj', Elena E. Espino², Glenda G. Torres², Mubashir M. Pervez ${ }^{2}$, Wesley W. Jones ${ }^{2}$, Yoonkyung Y. Chang ${ }^{3}$, Kiwon K. Lee ${ }^{2}$, James J. Grotta ${ }^{4}$, Tiffany T. Chang ${ }^{2}$, Huimahn H. Choi'.

${ }^{1} 1$ Department of Neurosurgery, University of Texas Health Science Center at Houston, Houston, USA, ${ }^{2}$ Department of Neurosurgery, University of Texas Health Science Center at Houston, Houston, USA, ${ }^{3}$ Department of Neurology, Ewha Womans University Mokdong Hospital, Seoul, Korea, Republic of, ${ }^{4}$ Memorial Hermann Hospital-Texas Medical Center, Houston, USA.

****Permission was not granted to print this abstract**** 


\section{Poster 189}

\section{Usefulness of Procalcitonin (PCT) to Differentiate between Infectious and Central Fever in Patients with Subarachnoid Hemorrhage}

Muhammad K. Athar, Umer U. Mukhtar, Umer U. Shoukat, David D. Boorman, Fred F. Rincon, Matthew M. Vibbert, Syed S. Shah, Jacqueline J. Urtecho, Jack J. Jallo.

Thomas Jefferson University Hospital, Philadelphia, PA, USA.

\section{Introduction:}

Fever is frequent in patients with subarachnoid hemorrhage (SAH). Differentiating infectious fever from central fever can be challenging. It is important to diagnose the cause of fever in the neurological intensive care unit (NICU) because of the detrimental effects of fever on brain injured patients. We hypothesized that procalcitonin (PCT) could be useful to distinguish central fever from infectious fever in patients with SAH.

\section{Methods:}

Prospective, chart review study conducted in the NICU between December 2012 and September 2015. Patients with SAH and fever $\left(\geq 101.0^{\circ} \mathrm{F}\right)$ who had PCT levels measured were included. Primary outcome was clinical infection defined as positive cultures (blood, urine, sputum, mini BAL, CSF, and C. difficle toxin) or infiltrate on chest X-ray within 3 days of onset of fever.

\section{Results:}

Sixty-two patients had temperature $\geq 101.0^{\circ} \mathrm{F}$, and another four patients had Arctic Sun reading $\leq 15$. Twenty-two patients had PCT value $\leq 0.1,28$ had PCT value between 0.1-0.5, and 16 had PCT $>0.5$. Out of 66 patients, 28 were positive for any culture: 3 with PCT $\leq 0.1,12$ with PCT $>0.1$ and $\leq 0.5$, and 13 with PCT >0.5. Using multiple logistic regression, PCT between $0.1-0.5$ had an odds ratio of $5.34(95 \% \mathrm{Cl}$ 1.20-23.73), PCT $>0.5$ had an odds ratio of 64.82 (Cl 7.03-597.71), and a maximum temperature odds ratio of $1.825(\mathrm{Cl} 1.095-3.042)$. Using PCT $>0.5$ alone had an odds ratio of $10.11(95 \% \mathrm{Cl} 2.51-40.74)$. For procalcitonin $>0.5$, specificity is $92.1 \%$, sensitivity $46.4 \%$, +PV $81.3 \%$, and -PV: $70.0 \%$ with a sample prevalence of $42.4 \%$. ROC Curve area: $78.2 \%$.

\section{Conclusions:}

PCT of $0.5 \mathrm{ng} / \mathrm{mL}$ or greater was demonstrated to be very useful for distinguishing infectious from central fever in SAH patients. The test has high specificity and NPV so it can be a valuable toll to rule out infectious fever in NICU. 


\title{
Poster 190
}

\section{Elevated Graeb Score is Associated With Loss of Independence after Aneurysmal Subarachnoid Hemorrhage}

\author{
Imad R. Khan ${ }^{1}$, Zaka Z. Ahmed ${ }^{1}$, Shakeel S. Tabish ${ }^{1}$, Haroon H. Majoka ${ }^{1}$, Sara S. Hefton ${ }^{1}$, Aashish A. \\ Anand $^{1}$, Christopher C. Melinosky ${ }^{1}$, Melissa M. Motta ${ }^{1}$, Wan-Tsu W. Chang ${ }^{1}$, Neeraj N. Badjatia', Gunjan \\ G. Parikh'2.
}

${ }^{1}$ Section of Neurocritical Care, Program in Trauma, Univ. of Maryland School of Medicine, Baltimore, MD, USA, ${ }^{2}$ Section of Neurocritical Care, Program in Trauma, Univ. of Maryland School of Medicine, Batlimore, MD, USA.

\section{Introduction:}

Intraventricular hemorrhage (IVH) due to subarachnoid hemorrhage (SAH) has been associated with fever, hydrocephalus, and shunt dependence. The modified Graeb Score (mGS) as an enhanced measure of intraventricular hemorrhage has been shown to correlate with modified Rankin outcomes in intracerebral hemorrhage $(\mathrm{ICH})$ as well as shunt dependency in SAH. We evaluated the mGS's association to complications during hospital stay and impact on functional independence at discharge in SAH patients.

\section{Methods:}

Retrospective review was performed of prospectively collected data for consecutive SAH patients enrolled into the University of Maryland Recovery After Cerebral Hemorrhage (REACH) Study. Hunt and Hess $(\mathrm{HH})$ grade, global cerebral edema (GCE), and infarct on admission was adjudicated weekly by a team of neurointensivists. mGS was calculated from each patient's admission CT scan and dichotomized according to a cutoff value based on the median value for our sample. Clinical complications during each admission were recorded, and independence of performing ADL's was obtained from physical and occupational therapy notes. Statistical analysis was performed using univariate and multivariate logistical regression.

\section{Results:}

Ninety-eight SAH patients from July 2014 to November 2015 were reviewed for this study. mGS was calculated in 83 patients and dichotomized based on a median cutoff value of 5 . HH, GCE, and admission infarcts were not found to be significantly associated with high mGS. On univariate analysis, elevated mGS was significantly associated with hospital acquired infections (UTI, pneumonia, and sepsis, $p<0.03$ ), fever $(p=0.02)$, hypotension $(p=0.002)$, hypernatremia $(p=0.046)$, symptomatic vasospasm $(p<0.03)$, and new infarct on CT $(p=0.01)$. On multivariate analysis, elevated mGS was associated with lack of independence with ADL's $(p=0.02)$.

\section{Conclusions:}

Severity of IVH as measured by mGS is associated with multiple in-hospital complications. The mGS can be used as an independent predictor of loss of independence of ADL's on discharge for patients with SAH. 


\title{
Poster 191
}

\section{Usefulness of UltraSound Lung Comets as a Sign of Interstitial Pulmonary Edema in Patients with High Grade Subarachnoid Hemorrhage (US-LUNG-COMET Study)}

\author{
Jason Siegel ${ }^{1}$, William W. Freeman ${ }^{2}$, Jose J. Diaz-Gomez ${ }^{3}$, Emily E. Edwards ${ }^{4}$.
}

\footnotetext{
${ }^{1}$ Mayo Clinic Florida Departments of Neurology and Neurocritical Care, Jacksonville, FL, USA, ${ }^{2}$ Mayo Clinic Florida Departments of Neurology, Neurocritical Care, and Neurosurgery, Jacksonville, FL, USA, ${ }^{3}$ Mayo Clinic Florida Departments of Neurocritical Care and Critical Care Medicine, Jacksonville, FL, USA, ${ }^{4}$ Mayo Clinic Florida Department of Neurology Clinical Studies Unit, Jacksonville, FL, USA.
}

\section{Introduction:}

Pulmonary complications represent half of the non-neurological medical deaths associated with aneurysmal subarachnoid hemorrhage (aSAH). Neurogenic pulmonary edema is a common complication, with an incidence of $8-20 \%$. Lung ultrasound (LUS) has shown promising data in identifying pulmonary edema, and can be obtained at bedside. There is scant prospective data, however, that also measures heart function via bedside echocardiogram.

\section{Methods:}

This is a pilot, prospective, observational study of patients admitted to the neurointensive care unit (NICU) with poor grade aSAH and intubation. Patients received a daily chest x-ray (CXR) as standard of care, as well as daily LUS assessment with visual estimation of the ejection fraction (EF), for the first 8 days after enrollment. Data collection also included demographics, routine blood tests, fluid balance, and vital signs.

\section{Results:}

Twenty-one patients were screened between January 2014 and December 2015, of which 19 were enrolled. The median age was 53 years (IQR 45.75-61.5 years). Demographically, 83.3\% were female and $61.1 \%$ were treated with coiling. There were 113 pairs of LUS and CXRs. When compared, LUS has a sensitivity of $94.7 \%$ and a negative predictive value of $97.5 \%$. Specificity was $41.5 \%$ and positive predictive value was $24.7 \%$. When patients had LUS signs of pulmonary edema, $73.7 \%$ had normal cardiac ejection fraction ( $>55 \%$ ) while $10.9 \%$ had $\mathrm{EF}<30 \%$.

\section{Conclusions:}

This study prospectively compared LUS with CXR in determining sensitivity, specificity, NPV and PPV of LUS. We found that LUS has good sensitivity and NPV but poor specificity and PPV when compared to CXR alone. Future work will incorporate not only CXR but the total clinical picture of pulmonary edema, which may increase specificity and PPV. We also found that most patients with suspicious LUS had normal EF. Future work will include other measures of cardiopulmonary failure (e.g. BNP, fluid balance, and $\mathrm{SaO} 2 / \mathrm{FIO} 2$ ratio). 
Poster 192

\title{
Propensity Score Analysis of the Impact of Corticosteroid Use on Delayed Cerebral Ischemia and Poor Functional Outcomes following Subarachnoid Hemorrhage
}

\author{
Nathaniel J. Mohney ${ }^{1}$, Venkatakrishna V. Rajajee ${ }^{2}$, Danielle D. Burghardt', Kyle K. Sheehan ${ }^{4}$, Jeffrey J. \\ Fletcher, Aditya A. Pandey ${ }^{4}$, B. Gregory B. Thompson ${ }^{4}$, Teresa T. Jacobs ${ }^{4}$, Craig C. Williamson ${ }^{4}$. \\ ${ }^{1}$ University of Michigan, Ann Arbor, MI, USA, ${ }^{2}$ University of Michigan Department of Neurosurgery, Ann \\ Arbor, MI, USA, ${ }^{3}$ University of Michigan, Pharmacy, Ann Arbor, MI, USA, ${ }^{4}$ University of Michigan, \\ Department of Neurosurgery, Ann Arbor, MI, USA, ${ }^{5}$ Bronson Neurocritical Care, Kalamazoo, MI, USA.
}

\section{Introduction:}

Laboratory studies suggest a link between inflammation, delayed cerebral ischemia (DCl) and brain injury following subarachnoid hemorrhage (SAH). While systemic corticosteroids may mitigate inflammation and promote fluid and salt retention following $\mathrm{SAH}$, there is limited evidence on the impact of corticosteroid administration on outcomes following SAH. Corticosteroids are frequently administered in clinical practice following SAH for the management of post-operative cerebral edema and refractory headache. Our goal was to examine the impact of corticosteroid use following $\mathrm{SAH}$ on the occurrence of $\mathrm{DCl}$ and poor functional outcome at discharge.

\section{Methods:}

Retrospective analysis of data from a single center SAH registry on patients admitted between 20102015 who survived $>24$ hours. A logistic regression model was created with multiple potential predictors of outcome and steroid use, and with corticosteroid use as the response variable. Patients were divided into quartiles based on the propensity score. The impact of corticosteroid use on the outcome of interest ( $\mathrm{DCl}$ then poor functional outcome at discharge) was then determined while controlling for the propensity score quartile. Co-variates in analysis included age, gender, ethnicity, history of diabetes or statin use, aneurysm location, aneurysmal vs non-aneurysmal bleed, treatment modality, Hunt Hess, modified Fisher.

\section{Results:}

A total of 440 patients with aneurysmal and nonaneurysmal SAH were included in this analysis. Corticosteroids were administered in $166(37.7 \%)$. DCl occurred in $122(27.7 \%)$. Poor outcome (mRS>3 at discharge) occurred $174(39.5 \%)$. Following propensity score analysis, corticosteroid use was not associated with $\mathrm{DCl}(\mathrm{p}=0.826)$ but was associated with a significant reduction in poor outcomes at discharge $(p=0.038$, OR $0.609,95 \%$ Cl 0.381-0.972).

\section{Conclusions:}

Corticosteroid use following SAH was not associated with a reduction in $\mathrm{DCl}$ but was associated with an approximately $40 \%$ reduction in the odds of poor functional outcome at discharge. A clinical trial of corticosteroids initiated in the early period following SAH may be warranted. 


\title{
Poster 193
}

\section{Continuous intra-arterial infusion of Nimodipine (CIAN) for treatment of severe refractory cerebral vasospasm (CV) after subarachnoid hemorrhage (SAH)- early use of multimodal neuromonitoring is the key for success}

\author{
Sylvia Bele ${ }^{1}$, Judith J. Scheitzach ${ }^{2}$, Andreas A. Hochreiter ${ }^{3}$, Martin M. Proescholdt ${ }^{2}$, Alexander A. \\ Brawanski .
}

\footnotetext{
${ }^{1}$ Regensburg University Medical Center/Dept. of Neurosurgery, Regensburg, Germany, ${ }^{2}$ Regensburg University Medical Center/ Dept. of Neurosurgery, Regensburg, Germany, ${ }^{3}$ Regensburg University Medical Center/ Dept. of Neurosurgery, Regensburg, Germany.
}

\section{Introduction:}

Besides the impact of the initial bleeding, CV remains the leading cause for mortality and morbidity after $\mathrm{SAH}$. We established CIAN as a treatment option for severe CV. But what is the key factor for a successful CIAN therapy?

\section{Methods:}

Data of 30 patients with CIAN were analyzed with regard to onset of multimodal neuromonitoring, if one or both hemispheres were monitored and for the integration of neuromonitoring values in decision making. Outcome was evaluated at hospital discharge and 6 months after SAH using the Glasgow Outcome Scale (GOS). CT-scans were reviewed for infarctions at time of discharge.

\section{Results:}

24 patients were in the favourable outcome group (GOS4-5), 6 patients showed unfavourable outcome (GOS 1-3). In 4 patients of the GOS 1-3 group neuromonitoring was implanted in the hemisphere with the highest transcranial doppler (TCD) values. Additional monitoring was installed contralaterally if TCDs increased. In 3 of those patients, contralateral PbtO2 values were ischemic and angiography revealed severe $\mathrm{CV}$ in the non CIAN treated hemisphere. CT scans of those 3 patients revealed significant infarctions in the hemisphere that was not initially monitored. The 24 patients with GOS 4-5 were monitored bilaterally at early timpoints and the values influenced decision making. CT scans showed only small infarctions but no territorial infarct was seen at discharge.

\section{Conclusions:}

A delay in bilateral multimodal neuromonitoring might facilitate delayed cerebral ischemia (DIC). This might be due to a delayed detection of a mismatch between oxygen supply and consumption. In addition severe CV is not always detectable in TCD and might thus be diagnosed too late to initiate a successful CIAN therapy. In contrast, new severe CV or relaspses of CV after stop of CIAN therapy are detected efficiently if bilateral neuromonitoring is in place and the values are acted upon accordingly. 


\section{Poster 194}

\section{Desmopressin for Prevention of Rebleeding in Subarachnoid Hemorrhage patients}

Charles L. Francoeur ${ }^{1}$, Michael M. Schmidt ${ }^{2}$, Jan J. Claassen ${ }^{2}$, Stephan S. Mayer ${ }^{3}$, Edward E. Connolly ${ }^{4}$, Sachin S. Agarwal', David D. Roh ${ }^{2}$, Mitchell M. Elkind ${ }^{2}$, Soojin S. Park².

${ }^{1} \mathrm{CHU}$ de Québec - Université Laval / Department of Anesthesiology and Critical Care, Québec, Canada, ${ }^{2}$ Columbia University / Department of Neurology, New York, NY, USA, ${ }^{3}$ Icahn School of Medicine at Mount Sinai / Department of Neurology, New York, NY, USA, ${ }^{4}$ Columbia University / Department of Neurological Surgery, New York, NY, USA.

\section{Introduction:}

Rebleeding remains a frequent and catastrophic complication leading to poor outcome after subarachnoid haemorrhage (SAH). Desmopressin (DDAVP) is known for its hemostatic effects and recent guidelines suggest that its use in patients exposed to antiplatelet agents may be helpful. We hypothesized that DDAVP administration at admission reduces the risk of rebleeding.

\section{Methods:}

We performed an observational cohort study of all patients with a documented SAH enrolled in the Columbia University SAH Outcome Project between January 2008 and August 2015. Rebleeding was defined by pre-specified clinical and radiographic criteria, excluding pre-hospital, intraprocedural, and post-repair events. We compared the rate of rebleeding among patients who were, or were not, treated with DDAVP $(0.3 \mu \mathrm{g} / \mathrm{kg})$ for suspected exposure of antiplatelet. Logistic regression was used to measure the association between treatment with desmopressin and risks of rebleeding.

\section{Results:}

Among $1087 \mathrm{SAH}$ patients, $12 \%$ were treated with DDAVP. The overall frequency of rebleeding was 8.2\%: $4.4 \%$ among those treated with desmopressin, compared to $8.8 \%$ among those not treated. After adjusting for antiplatelet use, Hunt and Hess grade and the presence of a large aneurysm (maximum diameter $>10 \mathrm{~mm}$ ), treatment with DDAVP was associated with a $62 \%$ reduction in the risks of rebleeding (adjusted OR 0.38 95\% C.I. [0.16, 0.92]). This association was independent of antiplatelet agent exposure. Antiplatelet use and platelet transfusion had no association with incidence of rebleeding. In our cohort, use of DDAVP represents a number needed to treat of 16.8 to prevent one event. Treatment with DDAVP was associated with a higher incidence of hyponatremia $(21 \%$ vs $15 \%)$, with no difference in incidence of thrombosis or in delayed cerebral ischemia.

\section{Conclusions:}

SAH patients treated with DDAVP had a lower risk of aneurysm rebleeding than those not treated. These findings justify future studies of DDAVP treatment as first line therapy for medical hemostasis optimization after SAH. 


\title{
Poster 195
}

\section{Cardiac Arrest in Patients with Aneurysmal Subarachnoid Hemorrhage: A Rare but Devastating Presenting Feature}

\author{
David J. Robinson ${ }^{1}$, Nicholas N. Morris ${ }^{2}$, Angela A. Velazquez ${ }^{2}$, Roh R. David ${ }^{2}$, Christine C. Falo ${ }^{2}$, Sachin \\ S. Agarwal', Soojin S. Park', Edward Sander E. Connolly', Jan J. Claassen ${ }^{2}$. \\ ${ }^{1}$ Columbia University Medical Center, Department of Neurology, New York, NY, USA, ${ }^{2}$ Columbia \\ University Department of Neurology, New York, NY, USA, ${ }^{3}$ Columbia University Department of \\ Neurosurgery, New York, NY, USA.
}

\section{Introduction:}

Outcomes of patients with subarachnoid hemorrhage (SAH) and cardiac arrest (CA) have each improved due to aggressive management. Prognosis of patients that present with a combination of the two is less clear, but assumed to be poor.

\section{Methods:}

All patients that suffered CA within 24 hours of SAH onset were identified from a prospectively collected observational case series of 1618 adults with $\mathrm{SAH}$. We compared demographics, clinical/imaging characteristics, and outcomes of those with and without CA in the setting of SAH using binary logistic regression.

\section{Results:}

Only $2 \%(\mathrm{~N}=38)$ of SAH patients had CA within 24 hours of the bleed. $55 \%(\mathrm{~N}=21)$ of those with CA had confirmed pulseless electrical activity (PEA)/asystole as the initial rhythm; all of these patients died while in the hospital. Three patients had a ventricular fibrillation (VFIB) arrest, and one of these patients survived with a good outcome (modified Rankin 2 at 3 months). The initial rhythm was unknown in $37 \%$ $(\mathrm{N}=14)$, and half of these patients survived. $79 \%$ of patients were comatose after the arrest, most of which died while in the hospital (28/30). Only 2 of the 8 noncomatose patients died. $31 \%(N=12)$ of the patients underwent cooling (goal temperatures 32-34). 37\% of deaths in our cohort were from withdrawal of life support $(\mathrm{N}=11)$. Increased aneurysm size (OR 1.08 for each $1 \mathrm{~mm}, 95 \% \mathrm{Cl} 1.01-1.15)$, amount of SAH (OR 4.97, 95\% Cl 1.77-16.24), and global cerebral edema (OR 3.35, Cl 1.06-9.65) were associated with risk of $\mathrm{CA}$.

\section{Conclusions:}

$\mathrm{CA}$ after SAH is most often due to PEA/asystole and outcomes are dismal despite aggressive treatment. Noncomatose patients and those with VFIB arrests may have a better prognosis. Acute herniation at the time of bleeding as indicated by large volume SAH and global cerebral edema may be the underlying mechanism of most early cardiac arrest in SAH patients. 
Poster 196

\title{
The effects of short versus longer duration prophylaxis of levetiracetam on cognitive / functional outcomes in aneurysmal subarachnoid hemorrhage and risk of development of delayed seizures
}

\author{
Tamara Majic, Dela D. Amoussou, Chrystal C. Reed, Asma A. Moheet.
}

Cedars Sinai Medical Center, Los Angeles, CA, USA.

\section{Introduction:}

Determine the effects of short versus longer duration prophylaxis of levetiracetam on cognitive / functional outcomes in aneurysmal subarachnoid hemorrhage $(\mathrm{aSAH})$ and risk of development of delayed seizures

\section{Methods:}

Chart review performed on 136 patients admitted from January 2010 to December 2015 with aSAH, who received levetiracetam seizure prophylaxis $500 \mathrm{mg} \mathrm{BID}$ or greater for $<3$ days versus 7 days or more. We compared the length of ICU stay, delta GCS at discharge, MRS (30 vs 90 days), and incidence of delayed seizures

\section{Results:}

There is no significant difference between the MRS at discharge for 3 versus $7+$ day of levetiracetam therapy $(3.8+/-1.8$ versus $3.7+/-1.05 ; p<0.453)$ Lengths of ICU stay for short-duration levetiracetam therapy was $10.6+/-4.86$ vs $12.77+/-6.54$ for long-duration $(P<0.378)$. Length of ICU stay in low dose was $12.4+/-6$ vs high dose $14.1+/-6.6$ days $(p<0.1)$. Preliminary data for early and late onset seizures suggests worse cognitive /functional outcome. All of the delayed seizures occurred with longer duration prophylaxis Preliminary data suggests delayed cerebral ischemia was universally present in patients with delayed seizures. The incident rate of $\mathrm{DCl}$ was higher in patients with early seizures $(40 \%)$ vs without seizures $(20 \%)$

\section{Conclusions:}

Preliminary data suggest there is no significant difference in cognitive /functional outcomes at discharge between low dose and high dose, and between short duration and long-duration levetiracetam therapy. Length of ICU stay is shorter in subjects treated with low dose levetiracetam vs high dose, which may suggest that a low dose levetiracetam may have a lower adverse effect profile. The presence of delayed ischemia may warrant a longer duration prophylaxis. The longer duration prophylaxis does not seem to reduce the incidence of delayed seizures, although a longer duration of study is warranted. 


\title{
Poster 197
}

\section{Aneurysmal Subarachnoid Hemorrhage Trial Randomizing Heparin (ASTROH): A Phase II Evaluation of Continuous Low-Dose Intravenous Heparin Infusion for Low Grade aSAH Patients with Large Hemorrhage Burden}

\author{
Robert F. James ${ }^{1}$, Enzo E. Fortuny ${ }^{1}$, Ann A. Jerde ${ }^{2}$, Zaid Z. Aljuboori ${ }^{1}$ J J. Mocco ${ }^{3}$, J Marc J. Simard ${ }^{4}$, \\ Kevin K. Sheth ${ }^{5}$.
}

\begin{abstract}
${ }^{1}$ Department of Neurosurgery, University of Louisville School of Medicine, Louisville, KY, USA, ${ }^{2}$ Clinical Trials Unit, University of Louisville School of Medicine, Louisville, KY, USA, ${ }^{3}$ Department of Neurosurgery, Mount Sinai Ichan School of Medicine, New York, NY, USA, ${ }^{4}$ Department of Neurosurgery, University of Maryland School of Medicine, Baltimore, MD, USA, ${ }^{5}$ Department of Neurology, Yale University School of Medicine, New Haven, CT, USA.
\end{abstract}

\section{Introduction:}

In spite of improvements in mortality and physical disability for aneurysmal subarachnoid hemorrhage (aSAH) patients over the last 30 years, survivors with minimal 'early brain injury' are still at significant risk for 'delayed brain injury' often attributable to the direct neurotoxic and neuroinflammatory influence of the initial hemorrhage burden. These processes can result in global brain atrophy and commonly manifests as new cognitive disability including deficits with memory, executive function, and language. Heparin exerts a wide range of interactions postulated to antagonize multiple pathophysiological mechanisms implicated in aSAH. Here we review low-dose IV heparin (LDIVH) as a promising treatment for preventing 'delayed brain injury' in aSAH survivors and inform on a new multi-center randomized trial.

\section{Methods:}

Recent studies evaluating LDIVH in aSAH are reviewed. The ASTROH study is an open-label, blindedadjudication, randomized phase II trial. The primary efficacy outcome is mean Montreal Cognitive Assessment (MoCA, 0-30, Normal 26-30) scores at the 90-day follow-up and 88 patients will be enrolled over 2 years at 9 academic medical centers. The primary safety outcome is any Major Bleeding or Clinically Relevant Non-Major Bleeding. One-year outcomes are also being assessed.

\section{Results:}

LDIVH significantly reduced neuroinflammation, demyelination, and transsynaptic apoptosis in a rat SAH model. In a retrospective study 43 LDIVH patients were compared to 43 well-matched controls. LDIVH subjects had $9 \%$ clinical vasospasm and $0 \%$ vasospasm related infarction compared to $47 \%$ and $21 \%$ respectively in controls $(P=0.0002$ and $P=0.003)$. In another retrospective cohort study LDIVH patients $(n=25)$ had mean MoCA of 26.4 compared to 22.7 in controls $(n=22)(P=0.013)$. Multivariate analysis confirmed LDIVH positively influenced MoCA scores when controlling for factors that negatively influenced cognition. The ASTROH study is active and enrolled its first subject in April, 2016.

\section{Conclusions:}

LDIVH is a promising treatment for aSAH and is currently being investigated in a multi-center randomized trial (ASTROH), NCT02501434. 


\title{
Poster 198
}

\section{Gastrointestinal Bleeding in Aneurysmal Subarachnoid Hemorrhage: Incidence, Risk Factors, and Short-Term Outcomes}

\author{
Manoj K. Mittal', Ashwath A. Kumar², Kavelin K. Rumalla². \\ ${ }^{1}$ University of Kansas Medical Center/Neurology, Kansas City, KS, USA, ${ }^{2}$ University of Missouri-Kansas \\ City/ School of Medicine, Kansas City, MO, USA.
}

\section{Introduction:}

The goal for our study was to understand the incidence, risk factors of gastrointestinal bleeding (GIB) in gastrointestinal bleeding (aSAH) patients and to determine the effect of GIB on in-hospital complications and outcomes.

\section{Methods:}

The Nationwide Inpatient Sample was searched from 2002 to 2011 to identify all aSAH patients (age $\geq$ 18 ) and a subset of GIB patients. Multivariate analyses were used to identify independent risk factors for GIB in aSAH patients and to determine the effect of this complication on other in-hospital complications and outcomes.

\section{Results:}

The incidence of GIB in aSAH hospitalizations $(\mathrm{N}=239,808)$ was 1,363 per 100,000 patients with $24.7 \%$ requiring blood transfusions. Multivariate independent predictors of GIB included: age 55-64 (OR: 1.60, 95\% Cl: 1.39-1.84), age 65-74 (OR: 1.86, 95\% Cl: 1.59-2.17), age 75+ (OR: 2.13, 95\% Cl: 1.78-2.55), male gender (OR: 1.40, 95\% Cl: 1.29-1.52), Medicaid insurance (OR: 1.41, 95\% Cl: 1.23-1.60), black race (OR: $1.41,95 \% \mathrm{Cl}: 1.26-1.57$ ), fluid/electrolyte disturbance (OR: 1.84, 95\% Cl: 1.69-2.01), renal failure (OR: 2.08, 95\% Cl: 1.72-2.53), coagulopathy (OR: $2.41,95 \% \mathrm{Cl}: 2.13-2.72$ ), and liver disease (OR: 3.18, 95\% Cl: 2.69-3.76) (all $p<0.0001$ ). In adjusted models, endovascular coiling increased the odds of GIB by $43 \%$ while aneurysm clipping decreased the odds by $31 \%(p<0.0001)$. GIB was a predictor of several in-hospital complications, including sepsis (OR: $1.53,95 \% \mathrm{Cl}: 1.31-1.78)$, cardiac arrest (OR: $1.58,95 \% \mathrm{Cl}: 1.31-1.90)$, deep vein thrombosis (OR: $1.70,95 \% \mathrm{Cl}: 1.49-1.94)$, pulmonary embolism (OR: 2.87, 95\% Cl: 2.04-4.03), and blood transfusion (OR: 3.46, 95\% Cl: 3.13-3.84). Patients afflicted with aSAH and GIB suffered an $81 \%$ and $39 \%$ increased likelihood of disability and mortality, respectively $(p<0.0001)$.

\section{Conclusions:}

GIB in aSAH is uncommon and is influenced by patient demographics and preexisting comorbidities and significantly increases disability and mortality. 


\title{
Poster 199
}

\section{Early Laboratory and Vital Sign Trends Predict Delayed Cerebral Ischemia after SAH}

\author{
Siddharth Biswal ${ }^{1}$, Eric E. Rosenthal ${ }^{2}$, Sahar S. Zafar ${ }^{1}$,Kathryn K. Connor ${ }^{1}$, Sophia S. Bechek ${ }^{1}$, Apeksha \\ A. Shenoy ${ }^{2}$, Emily E. Boyle', Michael M. Westover'. \\ ${ }^{1}$ Neurology, Boston, MA, USA, ${ }^{2}$ Neurology, Boston, USA.
}

\section{Introduction:}

Early identification of delayed cerebral ischemia (DCI) following aneurysmal subarachnoid hemorrhage $(\mathrm{SAH})$ could allow more effective intervention. Statistical methods that predict $\mathrm{DCl}$ using variables collected routinely during ICU care such as trends in vital signs and laboratory values have shown promise in recent studies. However, these studies have not all employed methods to guard against model overfitting. In this study we use cross validation to obtain minimally-biased estimates of the value of passively collected ICU variables for predicting $\mathrm{DCl}$.

\section{Methods:}

Early identification of delayed cerebral ischemia (DCI) following aneurysmal subarachnoid hemorrhage $(\mathrm{SAH})$ could allow more effective intervention. Statistical methods that predict $\mathrm{DCl}$ using variables collected routinely during ICU care such as trends in vital signs and laboratory values have shown promise in recent studies. However, these studies have not all employed methods to guard against model overfitting. In this study we use cross validation to obtain minimally-biased estimates of the value of passively collected ICU variables for predicting DCI.

\section{Results:}

$\mathrm{DCl}$ occurred in $34 \%$ of patients. Penalized logistic regression selected 9 features for inclusion in the final predictive model, derived from GCS, heart rate, mean aterial blood pressure, respiratory rate, SpO2, ventricular drainage, and sodium data. The mean AUC of the model was $83 \%$. Potentially clinically relevant (sensitivity, specificity) points on the ROC curve included $(73,89) \%$ and $(90,52) \%$.

\section{Conclusions:}

$\mathrm{DCl}$ occurred in $34 \%$ of patients. Penalized logistic regression selected 9 features for inclusion in the final predictive model, derived from GCS, heart rate, mean aterial blood pressure, respiratory rate, $\mathrm{SpO} 2$, ventricular drainage, and sodium data. The mean AUC of the model was $83 \%$. Potentially clinically relevant (sensitivity, specificity) points on the ROC curve included $(73,89) \%$ and $(90,52) \%$. 
Poster 200

\title{
HIGH PLASMA MICRORNA-26A IS ASSOCIATED WITH GOOD OUTCOME FOLLOWING SUBARACHNOID HEMORRHAGE
}

\author{
Sherry H. Chou ${ }^{1}$, Basak B. Icli ${ }^{2}$, Sarah S. Clark ${ }^{3}$, Gabriella G. Santos ${ }^{4}$, Farzaneh F. Sorond ${ }^{5}$, Liangee L. \\ Hsu $^{6}$, Steven S. Feske ${ }^{7}$, MingMing M. Ning ${ }^{8}$, Eng E. Lo ${ }^{9}$, Mark M. Feinberg ${ }^{10}$.
}

\footnotetext{
${ }^{1}$ University of Pittsburgh/Dept. of Critical Care Medicine, Neurology, \& Neurosurgery., Pittsburgh, PA, USA, ${ }^{2}$ Brigham and Women's Hospital/Harvard Medical School, Dept of Cardiology, Boston, MA, USA, ${ }^{3}$ Brigham and Women's Hospital, Boston, MA, USA, ${ }^{4}$ University of Massachusetts/Dept of Radiology, Boston, MA, USA, ${ }^{5}$ Northwestern University Feinberg School of Medicine/Dept of Neurology, Chicago, IL, USA, ${ }^{6}$ Brigham and Women's Hospital/Harvard Medical School, Dept of Radiology, Boston, MA, USA, ${ }^{7}$ Brigham and Women's Hospital/Harvard Medical School, Dept of Neurology, Boston, MA, USA, ${ }^{8}$ Massachusetts General Hospital/Harvard Medical School, Dept of Neurology, Boston, MA, USA, ${ }^{9}$ Massachusetts General Hospital/Harvard Medical School, Dept of Radiology, Boston, MA, USA, ${ }^{10}$ Brigham and Women's Hospital/Harvard Medical School Dept of Cardiology, Boston, MA, USA.
}

\section{Introduction:}

Subarachnoid hemorrhage (SAH) remains a highly morbid disease leading to $>27 \%$ of all stroke-related year of life lost before age 65 . Mechanisms of SAH-related early brain injury and vasospasm remain poorly understood, and there are no biomarkers that reliably predict long term outcome after SAH. MicroRNA ( $\mathrm{miR}$ )-26a is released in response to hypoxia and promotes angiogenesis. We hypothesize that higher levels of miR-26a is associated with outcome in human SAH.

\section{Methods:}

We prospectively enrolled consecutive SAH subjects, banked serial CSF/plasma samples, and evaluated functional outcome by modified Rankin scores (mRS) via scripted telephone follow-up every 3 months. Good functional outcome is defined as $\mathrm{mRS} 50 \%$ reduction in caliber of any vessel on post-SAH day 7 cerebral angiogram. In 56 SAH subjects we compared CSF and plasma miR-26a by quantitative PCR on post-SAH days 1, 3 and 5 between outcome groups. Data are normalized using log-transformation and then compared using student's t-test. Logistic regression is used to adjust for known confounders.

\section{Results:}

Study population has mean age of $55.62 \%$ has Hunt and Hess $(\mathrm{HH})$ grade $>3$. Good outcome at 6 months is associated with higher plasma miR-26a levels on post-SAH day $3(p=0.0007)$ and day 5 $(p=0.04)$. After adjusting for important predictors of outcome ( $\mathrm{HH}$ grade; age), plasma miR-26a on post$\mathrm{SAH}$ day 3 remains strongly associated with outcome $(p<0.0001)$. Plasma miR-26a levels were not associated with vasospasm. MiR-26a is present in CSF and is elevated in SAH compared to controls $(p<0.0001)$, but CSF miR-26a showed no association with functional outcome or vasospasm status.

\section{Conclusions:}

Higher plasma miR-26a level at post-SAH day 3 is independently associated with 6-month SAH outcome. Mechanistic experiments are necessary to determine whether miR-26a expression is neuro- protective in $\mathrm{SAH}$. Validation studies in larger, independent cohorts are necessary to validate miRNA-26a as a prognostic SAH biomarker. 


\title{
Poster 201
}

\section{Enhanced Renal Clearance in Patients with Aneurysmal Subarachnoid Hemorrhage}

\author{
Kathryn A. Morbitzer ${ }^{1}$, J. Dedrick J. Jordan ${ }^{2}$, Kelly K. Sullivan ${ }^{3}$, Emily E. Durr ${ }^{3}$, Casey C. Olm-Shipman ${ }^{4}$,
} Denise D. Rhoney ${ }^{1}$.

${ }^{1}$ UNC Eshelman School of Pharmacy, Chapel Hill, NC, USA, ${ }^{2}$ UNC School of Medicine, Department of Neurology, Chapel Hill, NC, USA, ${ }^{3}$ UNC Hospitals, Department of Pharmacy, Chapel Hill, NC, USA, ${ }^{4}$ UNC School of Medicine, Department of Neurology, Chapel Hill, NY, USA.

\section{Introduction:}

Accurate assessment of renal function remains a unique challenge in patients with aneurysmal subarachnoid hemorrhage (aSAH). Mathematical estimates of creatinine clearance $(\mathrm{CrCl})$ routinely used are often inaccurate in this setting. Patients with aSAH have been shown to exhibit a hyperdynamic response leading to an enhanced renal clearance. No studies exist evaluating the directly measured creatinine clearance of patients with aSAH over time.

\section{Methods:}

This was a single-center prospective observational study of adult patients with aSAH admitted to the NSICU between January 2015 and July 2015. Eight-hour urinary creatinine clearances were performed daily to directly measure $\mathrm{CrCl}$ until the patient no longer had a foley catheter or the patient left the NSICU. Urinary $\mathrm{CrCl}$ were compared with routine estimated $\mathrm{CrCl}$ based on the Cockcroft-Gault equation. Statistical significance was defined as $p$-value $<0.05$.

\section{Results:}

Fifty patients with aSAH were enrolled in the study. The study sample was $68 \%$ female with a mean age of $57.2 \pm 10.7$ years. The median Hunt and Hess grade was 3 (IQR 2-4) and the median modified Fisher grade was 3 (IQR 3-4). Additionally, the median admission GCS was 12.5 (IQR 6-14) and median admission SOFA score was 2 (IQR 2-4). The mean urinary $\mathrm{CrCl}$ over the study period was $147.9 \pm 50.2$ $\mathrm{mL} / \mathrm{min} / 1.73 \mathrm{~m} 2$. This differed significantly from the estimated $\mathrm{CrCl}$ over the study period of $109.1 \pm 32.7$ $\mathrm{mL} / \mathrm{min} / 1.73 \mathrm{~m} 2(p<0.05)$. Additionally, the mean urinary $\mathrm{CrCl}$ was significantly higher than the estimated $\mathrm{CrCl}$ each individual study day with a maximum urinary $\mathrm{CrCl}$ of $156.3 \mathrm{~mL} / \mathrm{min} / 1.73 \mathrm{~m} 2$ on Day 9.

\section{Conclusions:}

Patients with aSAH consistently experienced urinary $\mathrm{CrCl}$ greater than estimated $\mathrm{CrCl}$ predicted based on the Cockcroft-Gault equation. As renally eliminated medications are routinely dosed based on mathematical estimates of renal function, further study is needed to optimize medication regimens in this patient population to prevent underexposure. 


\title{
Poster 202
}

\section{Enteral Feeding Increases Brain Interstitial Glucose Levels In Poor-Grade Subarachnoid Hemorrhage Patients}

\author{
Mario Kofler ${ }^{1}$, Alois A. Schiefecker ${ }^{1}$, Ronny R. Beer ${ }^{1}$, Chun Fai C. Cheah ${ }^{2}$, Paul P. Rhomberg ${ }^{3}$, Bettina B. \\ Pfausler ${ }^{1}$, Claudius C. Thomé ${ }^{4}$, Erich E. Schmutzhard ${ }^{1}$, Raimund R. Helbok'. \\ ${ }^{1}$ Department of Neurology, Medical University of Innsbruck, Innsbruck, Austria, ${ }^{2}$ Department of \\ Neurology, Hospital Pulau Pinang, Jalan Residensi, Georgetown, Pulau Pinang, Malaysia, ${ }^{3}$ Department \\ of Neuroradiology, Medical University of Innsbruck, Innsbruck, Austria, ${ }^{4}$ Department of Neurosurgery, \\ Medical University of Innsbruck, Innsbruck, Austria.
}

\section{Introduction:}

Low interstitial cerebral glucose-levels assessed by cerebral microdialysis (CMD) are associated with poor functional outcome in aneurysmal subarachnoid hemorrhage (aSAH) patients. Interventions aiming to increase CMD-glucose have not been studied so far. We sought to analyze the effect of enteral feeding on CMD-glucose and cerebral energy metabolism.

\section{Methods:}

Twenty-six consecutive aSAH patients undergoing multimodal neuromonitoring including CMD were studied. Interventions of full-strength enteral nutrition (EN) after $>4$ hours without any feeding preceding EN were identified. Parameters of systemic and cerebral metabolism and insulin dose were time-locked and analyzed together with continuous variables to study the effect of EN on brain metabolism (glucose, lactate, pyruvate and glutamate).

\section{Results:}

Out of 208 interventions in total, 145 were excluded because of simultaneous parenteral supplementation or missing values, leaving 63 interventions in 17 patients eligible for analysis. The mean EN-kcal per intervention was $472.4 \pm 10.7 \mathrm{kcal}(1.15 \pm 0.034 \mathrm{kcal} / \mathrm{kg} / \mathrm{h})$. CMD-glucose significantly increased from $1.59 \pm 0.13 \mathrm{mmol} / \mathrm{l}$ to a maximum of $2.03 \pm 0.2 \mathrm{mmol} / \mathrm{l}(\mathrm{p}<0.001)$ during $E N$, independent of cerebral perfusion pressure (CPP), baseline serum and brain glucose levels, the baseline metabolic profile [brain metabolic distress (LPR over 40) or mitochondrial dysfunction (LPR over 30 and pyruvate over 70umol/l)] and independent of the insulin dose given during the intervention. The increase of CMD-glucose was strongly dependent on the delta increase of serum glucose (median $31 \mathrm{mg} / \mathrm{dl}$, IQR $7-46 \mathrm{mg} / \mathrm{dl}$ ) observed during the intervention $(p<0.01)$. Although probe location influenced absolute CMD-glucose-levels $(p<0.001)$, significant increases were even observed in perilesional brain tissue $(p<0.001)$. No change in CMD-lactate, CMD-pyruvate, CMD-LPR or CMD-glutamate levels were observed ( $p$ over 0.4).

\section{Conclusions:}

Brain glucose levels increased during enteral feeding independent of CPP, baseline glucose levels, insulin administration, and probe location. Despite this increase, no additional metabolic improvement was observed. The clinical benefit of interventions targeting at higher cerebral glucose levels $(>0.7 \mathrm{mmol} / \mathrm{l})$ needs to be investigated in a prospective approach. 
Poster 203

\section{Agitation after subarachnoid hemorrhage: a frequent omen of hospital complications associated with worse outcomes}

Michael E. Reznik ${ }^{1}$, J. Michael J. Schmidt ${ }^{1}$, Ali A. Mahta ${ }^{1}$, Christine C. Lesch ${ }^{2}$, David D. Roh ${ }^{1}$, Sachin S. Agarwal $^{1}$, Soojin S. Park', Jan J. Claassen ${ }^{1}$.

${ }^{1}$ Department of Neurology, Columbia University Medical Center, New York, NY, USA, ${ }^{2}$ Department of Pharmacy, Columbia University Medical Center, New York, NY, USA.

\section{Introduction:}

Agitated delirium is frequently encountered after acute brain injury, but data is limited in patients with subarachnoid hemorrhage (SAH). We examined the incidence, risk factors, and consequences of agitation in these patients.

\section{Methods:}

A retrospective analysis was conducted of all patients admitted with spontaneous SAH from 1/2011$12 / 2015$ who were part of a prospective observational cohort study. Patients were screened for agitation via records of antipsychotic or dexmedetomidine administration, and agitation was confirmed via chart review for evidence of relevant symptoms. Risk factors and hospital complications were predefined by the study team. Outcome was assessed at 12 months using the modified Rankin Scale (mRS), Telephone Interview for Cognitive Status (TICS), and Lawton-IADL score.

\section{Results:}

Agitation developed in 52 of 309 patients (16.8\%) and was most common in the first 72 hours after admission, and in patients with Hunt and Hess grades 3 and 4. Agitated patients were significantly more likely to develop multiple hospital complications, and in half of these patients a complication appeared to occur within 24 hours of the onset of agitation. Patients with agitation had increased ICU and hospital lengths of stay, but this was not significant after controlling for other predictors of length of stay. For patients with Hunt and Hess grades 1-4, agitation was not independently associated with functional outcome $(m R S>3$ ) or cognitive impairment (TICS $\leq 30)$, but IADLs were significantly more likely to be impaired at 12 months compared to those without agitation after controlling for other predictors (Lawton $>8 ; p=0.03$, OR $2.7,95 \% \mathrm{Cl} 1.1-6.8)$.

\section{Conclusions:}

Patients with SAH frequently experience agitation requiring medical treatment, especially early in their clinical course, and especially in non-comatose patients with higher clinical grades. Agitation is also associated with the development of multiple hospital complications, and may have an independent impact on long-term outcomes. 


\title{
Poster 204
}

\section{Extended Course of Levetiracetam is Associated with Loss of Independence and Delirium after Aneurysmal Subarachnoid Hemorrhage}

\author{
Sara Hefton ${ }^{1}$, Michael M. Armahizer ${ }^{2}$, Shakeel S. Ahmad Tabish ${ }^{1}$, Sundeep S. Bojedla ${ }^{3}$, Zaka Z. Ahmed ${ }^{1}$, \\ Nicholas N. Schluterman ${ }^{4}$, Joseph J. Haymore ${ }^{1}$, Wendy W. Chang ${ }^{1}$, Melissa M. Motta ${ }^{1}$, Neeraj N. \\ Badjatia ${ }^{1}$, Gunjan G. Parikh". \\ ${ }^{1}$ Neurocritical Care, Program in Trauma, Univ. of Maryland School of Medicine, Baltimore, MD, USA, ${ }^{2}$ \\ Department of Pharmacy Services, University of Maryland Medical Center, Baltimore, MD, USA, ${ }^{3}$ \\ Department of Neurology, University of Maryland School of Medicine, Baltimore, MD, USA, ${ }^{4}$ Dept. of \\ Epidemiology and Public Health, Univ. of Maryland School of Medicine, Baltimore, MD, USA.
}

\section{Introduction:}

Seizures after subarachnoid hemorrhage (SAH) are a frequent complication. SAH patients are typically prescribed prophylactic anti-epileptic drugs (AED) for three to seven days. Phenytoin has fallen out of favor as AED prophylaxis due to its association with worsened outcome as well as drug interactions. Newer AEDs including levetiracetam are more commonly used despite an incomplete understanding of their effect on outcome.

\section{Methods:}

Retrospective analysis was performed of prospectively collected data for 97 consecutive SAH patients enrolled into the University of Maryland REcovery After Cerebral Hemorrhage (REACH) Study between $6 / 30 / 2014$ and $11 / 27 / 2015$. Hunt-Hess $(\mathrm{HH})$ and modified Fisher score (mFS) was adjudicated weekly by a team of neurointensivists. Retrospective analysis of cumulative dose of levetiracetam was divided into groups of low-dose $(=12,000 \mathrm{mg})$ using the median as a cutoff. Concordance and discordance was noted. Pearson Chi-Square was used. Association of levetiracetam dose and quetiapine use as a surrogate of in-hospital delirium was also investigated. Multi-variate logistic regression was used to determine predictors of ability to perform activities of daily living (ADLs) in 78 survivors.

\section{Results:}

97 aSAH patients from July 2014 to November 2015 were reviewed for this study. Cumulative levetiracetam dose was calculated in 89 patients and dichotomized into high-dose $(>=12,000 \mathrm{mg})$ or lowdose groups. Hunt-Hess was found to be significantly associated with high-dose levetiracetam. On multivariate analysis, high-dose levetiracetam was associated with lack of independence in ADLs $(p=0.011)$. There is a trend towards increased use of quetiapine in the high-dose levetiracetam group. Full analysis will be provided at time of presentation.

\section{Conclusions:}

An extended course of levetiracetam is an independent predictor of loss of independence in activities of daily living after SAH. There is also a trend toward increased delirium. Larger, prospective studies are necessary for a more complete understanding of the impact of seizure prophylaxis on functional outcome after subarachnoid hemorrhage. 


\title{
Poster 205
}

\section{Intra-Aortic Balloon Pump Counterpulsation in patients with SAH and Neurogenic Stunned Myocardium: A Case Series and Review of the Literature.}

\author{
Matthew Sharrock, Sanjeev S. Keshary, Michael M. Abraham, Manoj M. Mittal.
}

Neurology, Kansas City, USA.

\section{Introduction:}

Intra-aortic balloon pump (IABP) counterpulsation has been used to maximize cerebral blood flow in patients with subarachnoid hemorrhage (SAH), refractory vasospasm and evidence of cardiac dysfunction. Neurogenic stunned myocardium (NSM) presents with mild troponin leak, EKG changes and LV dysfunction. We present 9 cases with SAH, vasospasm and IABP placement, including 7 cases with NSM. We also reviewed the literature with the goal of examining the safety of IABP for cardiac dysfunction after $\mathrm{SAH}$, outcomes and selection criteria for its use.

\section{Methods:}

We searched for cases of SAH and IABP placement at the University of Kansas Medical Center (KUMC) from 2008 to 2015. 9 patients met criteria and all had a secured aneurysm, refractory vasospasm and echocardiograms prior to IABP placement. We collected demographics, vitals, EKG, troponin, medications, IABP and ICU complications, discharge and follow-up mRS.

\section{Results:}

Average age was 47.6 patients had global LV dysfunction, 1 partial akinesis and 2 mildly depressed EF. 9 had EKG changes, 8 mild troponin leak. $7 / 9$ patients had a poor outcome ( $m R S>3$ ) at discharge. However, at follow-up only $1 / 8$ of our patients had a poor outcome. Literature review identified 17 patients selected for IABP due to cardiac dysfunction, only three with evidence of NSM. 6/17 had IABP or ICU complications and $7 / 17$ had a poor outcome at longest follow-up.

\section{Conclusions:}

Our results indicate that patients that have IABP placement in the setting of $\mathrm{SAH}$, vasospasm and cardiac dysfunction may have a good outcome if they are younger, have evidence of reversible NSM and avoid ICU complications including PE, UTI and Sepsis. The patients level of mobility and independence at discharge may not be indicative of overall functional improvement. 
Poster 206

\title{
Use of Milrinone for the Management of Delayed Cerebral Ischemia Following Non-Traumatic Sub -Arachnoid hemorrhage - A Systematic Review
}

\author{
Eyad Althenayan ${ }^{1}$, Derek D. Debicki ${ }^{1}$, Ron R. Butler ${ }^{2}$, Teneille T. Gofton ${ }^{2}$, Loretta L. Norton ${ }^{2}$, Michael M. \\ Sharpe ${ }^{2}$, Bryan B. Young ${ }^{3}$. \\ ${ }^{1}$ Western University, London, Ontario, Canada, London, Canada, ${ }^{2}$ Western University, London, Canada , \\ ${ }^{3}$ Neurology, Owen Sound, Canada.
}

\section{Introduction:}

A significant complication of non-traumatic sub-arachnoid hemorrhage (NT-SAH) is the development of delayed cerebral ischemia associated with cerebral vasospasm. Milrinone, an inotrope and a phosphodiesterase inhibitor, has been used intravenously, intra-thecally and intra-arterially as a delayed cerebral ischemia treatment and prophylaxis. The purpose of the current study is to systematically review the available evidence on its efficacy for that indication.

\section{Methods:}

Articles from MEDLINE, EMBASE, Cochrane Library, clinicaltrials.gov, reference lists of relevant articles, and gray literature were searched. Study selection criteria were used and strength of evidence was graded. Neurological outcomes and side effects were assessed.

\section{Results:}

Of 158 articles identified, 18 studies met the selection criteria and analyzed. The level of evidence varied and was generally low.

\section{Conclusions:}

This systematic review helped determine the current state of evidence for the efficacy and safety of Milrinone in the management of delayed cerebral ischemia in the context of NT-SAH. The available evidence is promising but of generally low quality suggesting the need for a randomized controlled trial. 
Poster 207

\title{
Admission Mean Platelet Volume As A Predictor Of Subarachnoid Hemorrhage Occurrence In Diabetics And Non-Diabetics
}

\author{
Emmaculate M. Fields ${ }^{1}$, Lane L. Tinsley ${ }^{1}$, Ayesha A. Vohra ${ }^{1}$, Lori L. Yearout ${ }^{2}$, David D. Thompson ${ }^{2}$, \\ Bappaditya B. Ray'.
}

${ }^{1}$ The University of Oklahoma Health Sciences Center/ Neurology, Oklahoma City, OK, USA, ${ }^{2}$ The University of Oklahoma Health Sciences Center/ Biostatistics and Epidemiology, Oklahoma City, OK, USA.

\section{Introduction:}

Mean platelet volume (MPV) is a common daily laboratory investigation in subarachnoid hemorrhage (SAH) patients. Higher MPV is a reported association after acute cardiovascular/cerebrovascular diseases and is postulated to signify increased systemic thrombogenicity. Similarly, diabetics have elevated MPV suggestive of associated vasculopathic complications through increased thrombogenicity. In our retrospective cohort of SAH patients we observed that diabetic patients were more likely to present with non-aneurysmal SAH (naSAH) as compared to aneurysmal SAH (aSAH). Hence, we investigated if $\mathrm{MPV}$, marker for thrombogenicity, would predict occurrence of naSAH suggestive of its role in diabetic vasculopathy.

\section{Methods:}

We reviewed charts of patients admitted with the diagnosis of SAH between January 2011 and December 2015. We compared proportions using Fisher's exact tests, and constructed ROC curves to find threshold values for admission MPV that had the best combination of sensitivity and specificity to predict naSAH versus aSAH.

\section{Results:}

Of the 237 patients who met the inclusion criteria 200 were aSAH. Diabetic patients who presented with naSAH had better glycemic control than those with aSAH [6/13 (46.2\%) vs $3 / 24(12.5 \%)$; $p=0.02]$. In non diabetic patients, a threshold value for MPV of $8.97 \mathrm{fL}$ yielded the best combination of sensitivity and specificity to predict aSAH vs naSAH (AUC $=0.6 ; 95 \% \mathrm{Cl} 0.5,0.7)$. Using this threshold, $\mathrm{SAH}$ is more likely to be non-aneurysmal as compared to aneurysmal [21/34 (61.8\%) vs 66/166 (39.8\%), $p=0.02]$. Similar MPV association was not observed among diabetic patients presenting with SAH. Mean MPV at admission did not differ between diabetic patients who presented with aSAH (9.1fL) and those who presented with naSAH (8.9fL, $p=0.36)$.

\section{Conclusions:}

Non-diabetic patients with higher MPV of $8.97 \mathrm{fL}$ and above are more likely to have naSAH. But, admission MPV is likely to have several other determinants and cannot be reliably used as a marker for thrombogenicity/ vasculopathy in diabetic patients presenting with $\mathrm{SAH}$. 


\title{
Poster 208
}

\section{Blood lactate variability: a strong independent predictor of neurological outcomes in patients with aneurysmal subarachnoid hemorrhage}

\author{
Tomoya Okazaki ${ }^{1}$, Toru T. Hifumi ${ }^{1}$, Kenya K. Kawakita ${ }^{2}$, Arisa A. Manabe ${ }^{1}$, Hideyuki H. Hamaya ${ }^{1}$, \\ Natsuyo N. Shinohara ${ }^{1}$, Hajime H. Shishido ${ }^{1}$, Koshiro K. Takano ${ }^{1}$, Yuko Y. Abe ${ }^{1}$, Yasuhiro Y. Kuroda ${ }^{1}$. \\ ${ }^{1}$ Emergency Medical Center, Kagawa University Hospital, Miki, Japan, ${ }^{2}$ Emergency Medical Center, \\ Kagawa University Hospital, Miki, Jersey.
}

\section{Introduction:}

Blood lactate levels during intensive care unit (ICU) management of patients with aneurysmal subarachnoid hemorrhage (SAH) can be used as an indicator of not only volume status but also aerobic glycolysis caused by excessive catecholamine levels and impaired lactate clearance. To determine whether blood lactate variability (LV) can predict neurological outcomes in patients with $\mathrm{SAH}$, we assessed the standard deviation (SD) of blood lactate level of each patient during ICU stay.

\section{Methods:}

We retrospectively reviewed all patients at the age of 18 years or older who were consecutively hospitalized in Kagawa University Hospital with SAH and at least five arterial lactate measurements between January 1, 2009 and May 31, 2015. Patients were divided into two groups with a mean lactate cutoff level of $1.0 \mathrm{mmol} / \mathrm{L}$. Patients were further subdivided into three subgroups using quartiles of the SD of lactate level (quartiles 1, $2+3$, and 4). Univariate and multivariate analysis were performed to identify independent predictors of unfavorable neurological outcome.

\section{Results:}

Unfavorable neurological outcomes occurred in $44.2 \%$ of a total of 122 patients. In both groups, there were increases in unfavorable neurological outcomes with increasing SD of lactate (quartile 1, 25\%; quartile $2+3,34 \%$; quartile $4,50 \%$ and quartile $1,36 \%$; quartile $2+3,65 \%$; quartile $4,80 \%$ in the mean lactate $<1.0 \mathrm{mmol} / \mathrm{L}$ and mean lactate $\geq 1.0 \mathrm{mmol} / \mathrm{L}$ groups, respectively). Univariate analysis determined that SD of lactate, age, Hunt and Kosnik (H\&K) grade, and mean glucose level were significantly correlated with unfavorable neurological outcomes $(p<0.01)$. Multiple logistic regression analysis showed that SD of lactate (odds ratio, 9.07; 95\% confidence interval, 2.06-47.6, p < 0.01), age, and H\&K grade were independent predictors.

\section{Conclusions:}

This study demonstrated that increased LV was an independent predictor of unfavorable neurological outcomes in patients with SAH. 
Poster 209

\section{Use of Milrinone in the Treatment of Delayed Cerebral Ischemia due to Refractory Vasospasm: a Tertiary Academic Hospital Experience in Brazil}

Danyelle Sadala ${ }^{1}$, CAROLINA C. ROUANET ${ }^{1}$, EVA E. ROCHA ${ }^{1}$, VIVIAN V. Gagliardi ${ }^{1}$, MARAMELIA M. ALVES ${ }^{2}$, FLAVIO F. CARVALHO ${ }^{1}$, Raul R. Valiente ${ }^{1}$, Marcel M. Ken ${ }^{1}$, Leticia L. Duarte ${ }^{1}$, Gisele G. Silva ${ }^{1}$.

${ }^{1}$ UNIFESP, São Paulo, Brazil, ${ }^{2}$ UNIFESP, SAO PAULO, Brazil.

\section{Introduction:}

The main causes of mortality and morbidity after aneurysmal subarachnoid hemorrhage (SAHa) are rebleeding and delayed cerebral ischemia secondary to cerebral vasospasm. The use of milrinone, an inotropic and vasodilator agent, is described in as one option to treat vasospasm in patients with refractory symptoms. Our objective was to describe the experience of our neurocritical care service with the use of milrinone in accordance with the Montreal Protocol for patients with refractory vasospasm.

\section{Methods:}

A retrospective study based on data obtained from medical records of patients suffering from SAHa and refractory vasospasm treated with milrinone from February 2015 to February 2016.

\section{Results:}

From 70 SAHa patients admitted to our hospital during the study period, 8 were identified with refractory vasoespasm. The mean age of the patients treated with milrinone was $48.3+/ 313.6$ years. All patients were female and $50 \%$ of patients were pre-hypertensive. A total of $62 \%$ of the patients had Hunt-Hess scores between 1-3 and $75 \%$ scored 3 or 4 in the modified Fisher scale. Vasosespam was identified after a mean of $8.7+/-3.2$ days after SAHa and its mean duration was $13.3+/-9.2$ days. In $75 \%$ of the patients hypertension was induced with norepinephrine as an initial treatment. The mean duration of the treatment with milrinone was $6.8+/-4.6$ days. Two cases were treated with intra-arterial milrinone and angioplasty. The most common adverse event during the use of milrinone was hypotension (50\%). Death occurred in 2 patients. Favorable functional outcome at the discharge was observed in $37 \%$ of the cases.

\section{Conclusions:}

In conclusion, the use of milrinone seems to be a safe option in the treatment of delayed cerebral ischemia secondary to vasospasm, especially in services where the availability of endovascular treatment is not a routine. 


\section{Poster 210}

\section{Troponin Elevation for Multi-organ dysfunction in Subarachnoid Hemorrhage}

Sungho Ahn ${ }^{1}$, Jude P J. Savarraj ${ }^{1}$, Jin J. Park ${ }^{1}$, Mubashir M. Pervez ${ }^{2}$, Tiffany R T. Chang ${ }^{2}$, Dong H. D. $\mathrm{Kim}^{2}$, Arthur L A. Day ${ }^{2}$, H. Alex H. Choi ${ }^{2}$, Kiwon K. Lee ${ }^{2}$.

${ }^{1}$ Division of Neurocritical care, University of Texas Health Science Center at Houston, Houston, TX, USA,

${ }^{2}$ Division of Neurocritical care, University of Texas Health Science Center at Houston, Houston, USA.

\section{Introduction:}

Troponin, a marker of myocardial injury, frequently increases in acute stage of subarachnoid hemorrhage (SAH). However, pathomechanism and etiology of this elevation leading to poor outcomes remains uncertain. This study investigated the effect of troponin elevation on multi-organ dysfunction and outcomes in patients with SAH.

\section{Methods:}

We retrospectively analyzed medical data of the prospectively registered stroke patients who were admitted to the Neuroscience Intensive Care Unit from July 2013 to January 2016.

\section{Results:}

Among 307 patients, 209 patients were eligible for inclusion with investigation of serum troponin level at admission. Troponin elevation $(>0.04 \mathrm{ng} / \mathrm{dL})$ was detected in 71 patients $(23.1 \%)$. Patients with troponin elevation were older $(59.1 \pm 14.4$ vs $53.0 \pm 12.8$ years; $P<0.01)$ and more often had a loss of consciousness $(31.0 \%$ vs $16.7 \% ; P<0.01)$, symptomatic hydrocephalus $(42.3 \%$ vs $23.2 \% ; P=0.01)$, and a higher Hunt-Hess score (3.4 \pm 1.1 vs $2.6 \pm 0.9 ; P<0.01)$ and modified Fisher score $(3.4 \pm 0.6$ vs $3.0 \pm$ $0.7 ; \mathrm{P}<0.01)$ at ictal period. During hospitalization, patients with troponin elevation more often had a respiratory failure $(31.0 \%$ vs $18.1 \%$; $P<0.01)$, acute kidney injury $(28.2 \%$ vs $8.0 \% ; P<0.01)$ and liver dysfunction $(21.1 \%$ vs $8.0 \% ; P=0.01)$ and more often treated with vasopressure $(25.4 \%$ vs $13.8 \% ; P=$ $0.01)$ and longer duration of mechanical ventilation $(3.7 \pm 6.2$ vs $1.6 \pm 3.3$ day; $P=0.04)$ than those without troponin elevation.

\section{Conclusions:}

Troponin elevation in the acute stage of $\mathrm{SAH}$ is associated with multi-organ dysfunction. Thus troponin elevation might be useful for risk estimation for patients with $\mathrm{SAH}$. 


\section{Poster 211}

Postoperative management and outcome of aneurysmal subarachnoid hemorrhage patients with surgery in Saitama medical university international medical center -seven year survey-

Hiroki Sato.

Saitama Medical University International Medical Center/Cerebrovascular Surgery, Hidaka, Japan.

${ }^{* * * *}$ Permission was not granted to print this abstract**** 
Poster 212

\title{
Clinical Predictors of Outcomes in Patients with Aneurysmal Subarachnoid Hemorrhage
}

\author{
Eva N. Postma ${ }^{1}$, Sahar S. Zafar ${ }^{1}$, Siddharth S. Biswal', Apeksha A. Shenoy ${ }^{2}$, Kathryn K. O'Connor ${ }^{3}$, \\ Sophia S. Bechek ${ }^{4}$, Eric E. Rosenthal', Michael M. Westover'. \\ ${ }^{1}$ Massachusetts General Hospital and Vrije Universiteit Amsterdam, Boston, MA, USA, ${ }^{1}$ Massachusetts \\ General Hospital/Department of Neurology, Boston, MA, USA, ${ }^{3}$ Massachusetts General \\ Hospital/Department of Neurology, University Of Michigan, Boston, MA, USA, ${ }^{4}$ Massachusetts General \\ Hospital/Department of Neurology, Stanford University, Boston, MA, USA.
}

\section{Introduction:}

Aneurysmal subarachnoid hemorrhage (aSAH) is associated with mortality rates up to $20 \%$, and up to one half of survivors suffer from long term neurologic disability. Though several clinical scores have been developed to predict in-hospital mortality and long term outcomes, there is no universally accepted score. We sought to identify additional clinical parameters that predict outcomes to expand on existing work and create a new predictive model.

\section{Methods:}

We conducted a retrospective chart review of patients admitted with aSAH to a single neurocritical care unit from September 2011 to February 2016. We excluded patients with non-aneurysmal SAH (including trauma, AVMs, and mycotic aneurysms). Demographic and clinical variables collected included age, admission GCS, admission APACHE II score, Hunt and Hess score, presence of delayed cererbral ischemia, and hospital acquired infections. Our outcome measure was Glasgow Outcome Scale at discharge. We created a penalized logistic regression model to determine predictors of outcome. We assessed performance by estimating the area under the ROC curve (AUC).

\section{Results:}

Of 204 patients reviewed, 160 met inclusion criteria. The mean age of the cohort was 58 years. $69.4 \%$ $(n=111)$ of patients were female. The mean APACHE II score on admission was 14.4 (median 13.5). Majority of patients $(n=97,60.63 \%)$ had a discharge GOS of 3 . A combination of 5 predictors performed optimally: age, admission Apache II, GCS, use of mechanical ventilation and presence of hospital acquired infections. The mean AUC of the model was $89 \%$. At the point of maximum-accuracy on the ROC curve, the sensitivity was $96 \%$, and specificity was $68 \%$.

\section{Conclusions:}

Clinical features at admission and during hospitalization can predict outcomes in patients with aSAH. Clinical characteristics from the first few days of the hospital admission, such as hospital acquired infections, can be added to existing models, to improve outcome prediction scores. 
Poster 213

\title{
Dynamic Response of Neuronal Activity to Neurocritical Care Interventions Measured by Intracranial EEG following Subarachnoid Hemorrhage
}

\author{
Apeksha V. Shenoy ${ }^{* 1}$, Lucas L. Fleuren ${ }^{{ }^{2}}$, Siddharth S. Biswal ${ }^{3}$, Emily E. Boyle ${ }^{3}$, Valdery V. Moura ${ }^{3}$, \\ Sahar S. Zafar ${ }^{3}$, Aman A. Patel ${ }^{4}$, M. Brandon M. Westover ${ }^{3}$, Eric E. Rosenthal ${ }^{3}$.
}

${ }^{1}$ Massachusetts General Hospital, Boston, MA, USA, ${ }^{2}$ University Medical Center Utrecht Department of
Neurology, Utrecht, Netherlands, ${ }^{3}$ Massachusetts General Hospital, Department of Neurology, Boston,
MA, USA, ${ }^{4}$ Massachusetts General Hospital, Department of Neurosurgery, Boston, MA, USA.

\section{Introduction:}

Subarachnoid hemorrhage (SAH) patients may experience supply-demand mismatch of cerebral metabolism from seizures, vasospasm, cortical spreading depolarization, hydrocephalus, or cerebral edema. Previous studies have focused on non-neuronal measures of cerebral autoregulation. We examine the impact of various neurocritical interventions by examining anecdotally identified intracranial EEG (iEEG) responses considered clinically impactful as well as systematic examination of repeated interventions within patients.

\section{Methods:}

SAH patients of Hunt-Hess grade 3-5 underwent 1) clinical multimodality neuromonitoring utilizing brain tissue oxygen cerebral oximetry, cerebral blood flow, Spencer depth electrode, and fiberoptic ICP through a quad-lumen bolt, and/or 2) neurosurgical placement of a subdural electrode strip following informed consent using time-synchronized monitoring (CNS-210, Moberg Research). We reviewed clinician anecdotes of treatment responses to vasopressors, endovascular vasodilators, anti-seizure pharmacotherapy, nimodipine, and ventriculostomy adjustments. We then assessed each patient's response to multiple grouped interventions using spectral features including alpha-to-delta ratio (ADR) normalized to pre-intervention baseline (nADR). Paired t-tests and scatter plots, respectively, demonstrated the impact of interventions and blood pressure on nADR.

\section{Results:}

12 patients had available post-SAH iEEG data over 22 months. Of 5 patients with post-procedural brain tissue hypoxia/hypoperfusion responding to vasopressor/ventriculostomy management, four patients responded with an increase in iEEG alpha activity power. Two patients developed a decline in ADR associated with aSAH-related vasospasm, one who had EEG improvement after endovascular spasmolysis. Two patients developed scalp-negative iEEG seizures, both electroclinically improving with anti-seizure pharmacotherapy. Grouped interventions showed heterogeneous responses to vasopressors and one patient with a significant, repeated response. Nimodipine had no consistent discernible peri-dose impact on nADR. We display scatter plots showing the peri-intervention patient- specific correlation between mean arterial pressure and nADR.

\section{Conclusions:}

Patients with aneurysmal SAH may develop neuronal impairment rescuable by neurocritical care interventions. Our data show these responses are patient- and state-dependent, consistent with known models of dynamic SAH pathophysiology. 


\title{
Poster 214
}

\section{Delay in Presentation to Medical Care and Outcomes Following Nontraumatic Subarachnoid Hemorrhage}

\author{
Kyle M. Sheehan, Jenelle J. Rofe, Tanha T. Shah, Venkatakrishna V. Rajajee, Craig C. Williamson, Kyle \\ K. Sheehan.
}

University of Michigan Department of Neurosurgery, Ann Arbor, MI, USA.

\section{Introduction:}

Recent studies suggest that early brain injury (EBI), prior to the risk period for delayed cerebral ischemia $(\mathrm{DCl})$, may be an important determinant of outcome following subarachnoid hemorrhage (SAH). Potentially, early treatment measures such as control of intracranial pressure, blood pressure management and initiation of nimodipine may mitigate EBI. Our objective was to study the impact of delayed presentation to medical care on the occurrence of $\mathrm{DCl}$ and poor outcomes following $\mathrm{SAH}$.

\section{Methods:}

Retrospective analysis from a single center SAH registry. Patients admitted between 2011 and 2015 for nontraumatic SAH, who survived more than 24 hours were eligible for inclusion. [VR1] The explanatory variable of interest was time from symptom onset to diagnostic CT, dichotomized at 72 hours. Covariates included age, gender, ethnicity, Hunt-Hess grade, modified Fisher grade, hypertension, aneurysm location and treatment modality. The primary outcome of interest was poor functional outcome at discharge (defined as a modified Rankin Scale>3), secondary outcome was $\mathrm{DCl}$ (delayed cerebral infarction and/or clinical decline attributable to delayed ischemia in the risk period). Logistic regression models were constructed with the outcomes of interest as the response variables.

\section{Results:}

A total of 315 patients were included. The median time to diagnosis was 9.0 hours (interquartile range 3.9-16.9). Twenty-four patients (7.6\%) presented greater than 72 hours from onset. Poor functional outcome at discharge occurred in $138(43.8 \%)$ and $\mathrm{DCl}$ in $86(27.3 \%)$. Multivariate analysis revealed no association between delayed presentation and either $\mathrm{DCl}(\mathrm{P}=0.289)$ or poor functional outcome at discharge $(P=0.7)$. Hours from symptom onset to diagnosis as a continuous variable also did not reveal a significant association with $\mathrm{DCl}$ or poor functional outcome.

\section{Conclusions:}

Delayed presentation to medical care beyond 72 hours is not associated with either $\mathrm{DCl}$ or poor functional outcome at discharge following subarachnoid hemorrhage. 


\title{
Poster 215
}

\section{Prophylactic Normothermia with Endovascular Cooling Device Following Initial Therapeutic Hypothermia in Patients with Poor-Grade Subarachnoid Hemorrhage}

\author{
Hitoshi Kobata ${ }^{1}$, Takayoshi T. Negoro $^{2}$, Seiji S. Ogita ${ }^{1}$, Makiko M. Kawakami . \\ ${ }^{1}$ Osaka Mishima Emergency Critical Care Center, Takatsuki, Japan, ${ }^{2}$ Osaka Mishima Emergency Critical \\ Care Center, Takastuki, Japan.
}

\section{Introduction:}

To mitigate early brain damage in subarachnoid hemorrhage (SAH), we have been treating World Federation of Neurological Surgeons Grade (WFNS) Grade 5 patients with therapeutic hypothermia (TH) for 7 days immediately after onset. Management after rewarming was problematic since fever in SAH is associated with vasospasm and poor outcome. We studied the feasibility and safety of endovascular cooling to maintain prophylactic normothermia following initial TH in patients with severe SAH.

\section{Methods:}

$\mathrm{TH}$ (core body temperature $34.0^{\circ} \mathrm{C}$ ) was initiated, using surface cooling, immediately after the diagnosis of WFNS Grade $5 \mathrm{SAH}$ was made. The ruptured aneurysm was surgically clipped as soon as feasible. Around postoperative day 7 , after rewarming to $36^{\circ} \mathrm{C}$, an endovascular catheter with 2 cooling balloons (Cool Line ${ }^{\circledR}$ Catheter, Asahi Kasei ZOLL Medical Corp., Tokyo, Japan) was inserted into the internal jugular vein and connected to XP® Temperature Management System (Asahi Kasei ZOLL Medical Corp.) for 7 days. Prospectively collected data were analyzed.

\section{Results:}

Eleven patients ( 6 females; mean age, $63.8 \pm 6.4$ years [range, $50-73$ years]) were enrolled. Endovascular cooling was initiated $8.0 \pm 1.4$ days (range, $6-11$ days) after admission and continued for 6.7 days. Nine patients developed shivering with increased temperature and were given acetaminophen and dexmedetomidine. There was no evidence of vasospasm or additional cerebral infarction during endovascular cooling, and no catheter-related sepsis or thromboembolic event. After removal of the cooling catheter, vasospasm-related cerebral infarction and fatal bacterial meningitis related to spinal drainage occurred. Three-month outcomes were good recovery $(n=2)$, moderate disability $(n=4)$, severe disability $(n=2)$; vegetative state $(n=1)$, and death $(n=2)$.

\section{Conclusions:}

Elimination of fever burden in the first 14 days after onset was safe and feasible with combined surface and endovascular cooling in patients with WFNS Grade 5 SAH. 
Poster 216

\title{
Admission Lactic Acid Levels Are Associated With Clinical and Radiographic Grade of Aneurysmal Subarachnoid Hemorrhage and Predicts Inpatient Mortality
}

\author{
Roy A. Poblete, Benjamin B. Emanuel, Steven S. Cen.
}

University of Southern California/Keck Medical Center/Department of Neurology, Los Angeles, CA, USA .

\section{Introduction:}

Previous work has shown elevated sympathetic tone and metabolic crisis as part of aneurysmal subarachnoid hemorrhage (aSAH) pathophysiology. Serum lactate may serve as a marker for these disease processes. This study examines: 1) the relationship between admission lactate and the clinical and radiographic severity of aSAH, and 2 ) whether levels predict outcomes including vasospasm, delayed cerebral ischemia $(\mathrm{DCl})$, and inpatient mortality.

\section{Methods:}

This is a retrospective analysis of 75 consecutive aSAH patients with lactate drawn on admission. Patients with known infection were excluded. Patients with an elevated lactate $(>2.2 \mathrm{mmol} / \mathrm{L})$ were compared to those with normal levels. Differences between groups were compared using chi-square tests for categorical variables, and independent t-tests for continuous variables. Spearman correlations were calculated between lactate levels and mean values for continuous variables.

\section{Results:}

Elevations in lactate were associated with admission GCS, Hunt \& Hess $(\mathrm{HH})$ grade, Fisher score, serum white blood cell count (WBC), troponin I (TN), glucose, and ventilator-free days (VFD). Positive correlation was found between lactate and $\mathrm{HH}$, Fisher score, WBC, TN, and glucose. An inverse correlation existed between lactate and GCS, and VFD. Compared to survivors, non-survivors had significantly higher lactate levels. All results were considered significant with a $p$-value $<0.05$. No association between lactate and the development of vasospasm or DCl was found.

\section{Conclusions:}

Higher admission serum lactate is positively correlated with $\mathrm{HH}$ grade, Fisher score, serum WBC, TN and glucose, but negatively correlated with admission GCS and VFD. Presence of an elevated lactate was also predictive of inpatient mortality. This is the first report of correlation between early lactate and aSAH severity, and conflicts with prior results suggesting an association between lactic acid and the development of $\mathrm{DCl}$. Further studies are needed to determine whether lactate elevations relate to hypovolemia, acute inflammatory response, elevated sympathetic outflow, or other cause. 


\title{
Poster 217
}

\section{Factors associated with Failure of Targeted Temperature Management on Malignant Hemispheric Infarction}

\author{
Kyu Sun Yum¹, Won joo W. Jeong ${ }^{2}$, Sangkil S. Lee ${ }^{1}$, Moon-Ku M. Han ${ }^{1}$. \\ ${ }^{1}$ Seoul National University Bundang Hospital/Department of Neurology, Seongnam, Korea, Republic of, \\ ${ }^{2}$ Seoul National University Bundang Hospital/Department of Neurocritical care, Seongnam, Korea,
} Republic of.

\section{Introduction:}

Targeted temperature management(TTM) is known as effective treatment for cerebral edema in patients with malignant cerebral infarction with high osmotic pressure therapy. Some patients need decompressive hemicraniectomy or expire due to cerebral herniation after TTM. Hence this study was performed to determine associated factors in case of failure of TTM in patients with malignant cerebral infarction.

\section{Methods:}

From January 2011 to December 2014, a study was performed in patients with malignant cerebral infarction occurred within 24 hours at neurological intensive care unit. All patients were diagnosed through the brain CT or MRI. And they were treated by treatment guidelines of acute ischemic stroke with hyperosmotic fluid therapy and TTM. We defined failure of TTM to cases treated by decompressive hemicraniectomy or death due to brain herniation.

\section{Results:}

A total of 21 patients, TTM were failed in 12 patients. Failures of TTM were common in patients without recanalization after thrombolysis significantly $(8.3 \%$ vs $55.6 \%, \mathrm{p}=0.046)$. Leukocytosis on admission $(16.7 \%$ vs $0.0 \%, p=0.486)$, failures of maintaining target blood pressure within 24 hours $(33.3 \%$ vs $22.2 \%$, $p=0.659)$, and multiple territorial infarctions $(41.7 \%$ vs $11.1 \%, p=0.178)$ were common in patients with TTM failure, but there were no statistical significance. The volume of stroke was larger on patients with failure $(166.97 \mathrm{cc}(30.02-310.71)$ vs $110.28 \mathrm{cc}(0.65-213.51) \mathrm{p}=0.345)$, but there is no statistical significance.

\section{Conclusions:}

In conclusion, it may be related to the risk of brain herniation in case of failure of recanalization after acute middle cerebral artery occlusion, despite TTM. Although the study were not shown statistical significance due to the small number of patients, leukocytosis, failures of maintaining target blood pressure, larger volume of infarction and multiple territorial infarction may have relevance which is necessary to check through the further studies. 
Poster 218

\section{Amplitude-Integrated EEG for Prognostication of Cardiac Arrest Patients Under Extracorporeal Cardiopulmonary Resuscitation}

Hitoshi Kobata, Seji S. Ogita, Takayoshi T. Negoro, Makiko M. Kawakami, Hiroshi H. Hazui, Yoshiki Y. Yagi, Ryosuke R. Zushi, Hiroshi H. Akimoto.

Osaka Mishima Emergency Critical Care Center, Takatsuki, Japan.

\section{Introduction:}

A treatment bundle including extracorporeal cardiopulmonary resuscitation (ECPR) combined with targeted temperature management (TTM) may improve outcome of cardiac arrest (CA) patients, however, prognostication for these patients still remains challenging. We sought to examine the prognostic value of amplitude-integrated electroencephalogram (aEEG) for CA patients during ECPR and TTM.

\section{Methods:}

This was a single-center, retrospective analysis of adult CA patients treated with ECPR and TTM under aEEG monitoring with subhairline montage. Intra-arrest cooling was immediately initiated with cold fluid infusion and extracorporeal cooling method and maintained at $34{ }^{\circ} \mathrm{C}$ for $24 \mathrm{~h}$. Patents underwent intraaortic balloon pumping (IABP) and percutaneous coronary intervention ( $\mathrm{PCl}$ ) if needed. Neurological outcome was assessed with the Cerebral Performance Category (CPC) scale at hospital discharge.

\section{Results:}

ECPR was conducted in 17 patients (age 57.8 [24-84] years, $82 \%$ male) amongst 73 CA or post-CA comatose patients since November 2012. The initial cardiac rhythm was refractory ventricular fibrillation in 14 , pulseless electrical activity in 2, and asystole in 1 . The cause of CA was cardiogenic; 10 underwent $\mathrm{PCl}$ and 9 needed IABP support. Collapse-to-ECPR time was $34.3 \mathrm{~min}$. Initial aEEG patterns were; flat trace $(n=4)$; low voltage $(n=6)$; suppression-burst (SB) $(n=2)$; electrographic status epilepticus (ESE) $(n=1)$; continuous background $(n=1)$; unable to classify due to artifacts $(n=3)$. Five patients showed good recovery (CPC 1-2). Their aEEG pattern was continuous in 1, low voltage in 3 , and ESE in 1 . Among 3 patients with low voltage aEEG, 2 showed gradual improvement to continuous background and 1 showed rapid change into continuous background soon after the return of spontaneous circulation. Patients with ESE recovered after antiepileptic administration. ECPR was withdrawn in 6 patients based on clinical and prolonged flat aEEG findings.

\section{Conclusions:}

Continuous aEEG adds early prognostic information for CA patients with ECPR under TTM. 
Poster 219

\title{
Bedside Measurement of Suppression Ratio During Targeted Temperature Management After Cardiac Arrest: Agreement Between Medtronic Bispectral Index Monitor and Natus Full Montage EEG Using Persyst Magic Marker Software
}

\author{
Richard R. Riker ${ }^{1}$, Alexa A. Craig ${ }^{2}$, Louis L. Eubank ${ }^{1}$, Teresa T. May ${ }^{1}$, Barbara B. McCrum ${ }^{1}$, Lauren L. \\ Connolly", David D. Seder'. \\ ${ }^{1}$ Maine Medical Center / Neurocritical Care, Portland, ME, USA, ${ }^{2}$ Maine Medical Center / Neurology, \\ Portland, ME, USA.
}

\section{Introduction:}

The Suppression Ratio (SR) is a processed EEG variable estimating the percent of an EEG epoch (0100 ) that is suppressed. SR has been associated with neurologic outcome after several types of brain injury and using different technologies including full montage EEG recordings and simplified processed EEG monitors. We compared SR during targeted temperature management (TTM) after cardiac arrest, using two independent blinded assessment tools.

\section{Methods:}

A convenience sample of adult patients treated with TTM after cardiac arrest were enrolled to compare the SR determined by the Medtronic bispectral index monitor (amplitude $\leq 5$ microvolts for 0.5 seconds) and the full montage continuous EEG using Natus equipment with Persyst Magic-Marker software $(\leq 3 \mu \mathrm{V}$ for 0.5 seconds). Machine times were recorded to synchronize, and SR results were recorded once for each subject at a time without stimulation or artifact using correlation and Altman-Bland analysis.

\section{Results:}

20 adults were enrolled in this study with a median age of 56 years, $14(70 \%)$ were male. During SR collection, the temperature was $33.8^{\circ} \mathrm{C}$ and patients were receiving propofol at $20 \mathrm{mcg} / \mathrm{kg} / \mathrm{min}(\mathrm{n}=14)$, fentanyl at $37.5 \mathrm{mcg} / \mathrm{hr}(\mathrm{n}=17)$. The median (IQR) for Medtronic SR was 0 (0-46) for Persyst SR 1.7 (0.241). Comparing Medtronic and Persyst SR, the Spearman correlation was 0.88 ( $p<0.0001)$, and Altman Bland testing revealed a bias of 0.6 with $95 \%$ limits of agreement -3.7 to 4.8 .

\section{Conclusions:}

Bedside estimation of Suppression Ratio during TTM after cardiac arrest showed excellent agreement when measured with the Medtronic bispectral index monitor and the full montage Natus cEEG monitor using Persyst Magic Marker software. 
Poster 220

\title{
Temperature Management and Fever Avoidance in Critical Care Patients using an Esophageal Heat Transfer Device
}

\author{
Melissa I. Naiman ${ }^{1}$, Andrej A. Markota ${ }^{2}$, Ahmed A. Hegazy ${ }^{3}$, Erik E. Kulstad ${ }^{4}$. \\ ${ }^{1}$ University of Illinois at Chicago/CADRE, Chicago, IL, USA, ${ }^{2}$ University Medical Center Maribor/Medical \\ Intensive Care Unit, Maribor, Slovenia, ${ }^{3}$ University of Western Ontario/Department of Anesthesia and \\ Perioperative Medicine, Ontario, Canada, ${ }^{4}$ Advocate Christ Medical Center/Department of Emergency \\ Medicine, Oak Lawn, IL, USA.
}

\section{Introduction:}

Though the impact of therapeutic hypothermia on neurological outcomes remains controversial, there is strong evidence that pyrexia is detrimental. Posthypothermia fever experienced by cardiac arrest patients is of particular concern. This abstract examines the ability of an esophageal heat transfer device (EHTD) to maintain core temperature below $38^{\circ} \mathrm{C}$ in critical care patients, with a focus on posthypothermia fever in post cardiac arrest (PCA) patients.

\section{Methods:}

De-identified data for subjects who received temperature management using an EHTD were collected from two clinical sites. All patients were at least 18 years of age, weighed at least $40 \mathrm{~kg}$, and presented with a condition appropriate for active temperature management. Core temperature readings for each patient were recorded at least hourly; if measurements were recorded more frequently, temperature over an hour span was averaged. Patient data was analyzed to determine what proportion of measurements were above $38^{\circ} \mathrm{C}$.

\section{Results:}

Data from a total of 26 patients was collected, including 23 post-cardiac arrest patients and 3 fever reversal cases. A total of 934 core temperature measurement events (over an average of $35.9 \mathrm{~h}$ per patient) were included in the analysis. $927\left(99.3 \%\right.$ ) were below $38^{\circ} \mathrm{C}, 7$ recorded measurements exceeded $38^{\circ} \mathrm{C}$, and no data were recorded for 4 time points. Of the 254 measurements recorded posthypothermia, $252(99.2 \%)$ remained below $38^{\circ} \mathrm{C}$.

\section{Conclusions:}

Esophageal temperature modulation using an EHTD appears to be an effective method for fever prevention and reduction. Visual representations of the PCA subset showed an upward trend in temperature after 10-18 hours of maintaining target temperature, but before active cooling ended. This suggests that many of these patients might have become febrile in the absence of active temperature management. 
Poster 221

\section{Novel Esophageal Cooling Device for Therapeutic Normothermia}

Imad R. Khan ${ }^{1}$, Joseph J. Haymore ${ }^{1}$, Christopher C. Melinosky ${ }^{2}$, Mary Ann M. Bautista ${ }^{2}$, Wan-tsu W. Chang ${ }^{2}$, Melissa M. Motta ${ }^{2}$, Neeraj N. Badjatia ${ }^{2}$.

${ }^{1}$ Section of Neurocritical Care, Program in Trauma, Univ. of Maryland School of Medicine, Baltimore, MD, USA, ${ }^{2}$ Section of Neurocritical Care, Program in Trauma, Univ. of Maryland School of Medicine, Baltimore, MD, USA.

\section{Introduction:}

Achieving and maintaining normothermia (NT) after subarachnoid hemorrhage (SAH) or intracerebral hemorrhage $(\mathrm{ICH})$ often requires surface or intravascular cooling devices that are associated with a significant burden of shivering. We describe a new, closed loop esophageal cooling device (ECD: Esophageal Cooling Device; Advanced Cooling Therapy: Chicago, IL) connected to a Stryker MediTherm (Stryker Corporation: Kalamazoo, MI) system to induce NT $(37+/-0.50 \mathrm{C})$ and the shiver burden during the maintenance of NT.

\section{Methods:}

We enrolled mechanically-ventilated patients with SAH or ICH with refractory fever $(>38.3 \mathrm{C})$. Temperature and Bedside Shivering Assessment Scale (BSAS) were recorded every 15 minutes for the first 2 hours, then hourly. Success and time to NT, hourly temperature burden (TB: $>37.5 \mathrm{C} / \mathrm{hr}$.), percent time above $38 \mathrm{C}$, median BSAS and cumulative number of anti-shivering interventions per patient was recorded prospectively. All patients received magnesium, buspirone, and acetaminophen as baseline anti -shivering interventions.

\section{Results:}

Ten patients ( $7 \mathrm{ICH}, 3 \mathrm{SAH}$ ) were enrolled between October 2015 and April 2016. The median GCS at initiation was 6 (4-11), age 52+/-6 years old, BMI $25+/-6 \mathrm{~kg} / \mathrm{m} 2$, BSA $1.8+/-0.2 \mathrm{~m} 2$, and $70 \%$ were women. There was a temperature reduction at 120 minutes (mean $38.7 \mathrm{C}$ to $37.9 \mathrm{C}, \mathrm{p}=0.005$ ) and $90 \%$ of patients achieved NT (median time $=4.5$ hrs.; range: $0.5-38$ hours). NT was maintained for median 91 hours (range: 15 to 131 hours) with a TB of $0.05+/-0.5 \mathrm{C}^{*} \mathrm{hr}$, and $15 \%$ (range: $0-28 \%$ ) time above > $38.00 \mathrm{C} /$ patient. Median BSAS $=0$ with any shiver (BSAS>0) occurring $7.6 \%$ of the time. The median number of total shiver interventions per patient was $5(1-22)$ throughout the TTM time period. No device related complications were noted.

\section{Conclusions:}

The ECD successfully achieved and maintained NT with a low shiver burden and may be a feasible option for NT in this critically-ill population. 


\section{Poster 222}

Incidence, patterns, and risk factors of ischemic stroke after targeted temperature management for cardiac arrest

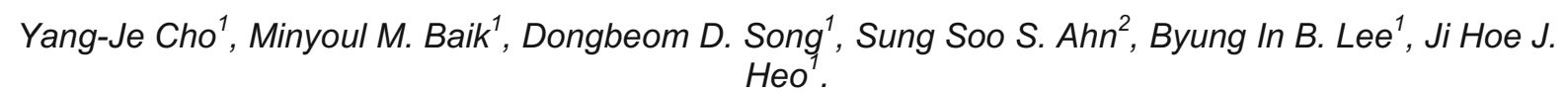

${ }^{1}$ Department of Neurology, Yonsei University College of Medicine, Seoul, Korea, Republic of, ${ }^{2}$ Department of Radiology, Yonsei University College of Medicine, Seoul, Korea, Republic of.

****Permission was not granted to print this abstract**** 
Poster 223

\title{
Performance of the EMCOOLs Surface Cooling System For Acute Fever Control In Neurocritical Care Patients
}

\author{
Stephen A. Griffiths ${ }^{1}$, Charles C. Francoeur ${ }^{1}$, Errol E. Gordon ${ }^{2}$, Neha N. Dangayach ${ }^{2}$, Danielle D. \\ Wheelwright ${ }^{1}$, Anil A. Ramineni, Javaad J. Ahmad ${ }^{3}$, Stephan S. Mayer ${ }^{1}$. \\ ${ }^{1}$ Icahn School of Medicine at Mount Sinai / Institute of Critical Care Medicine, New York, NY, USA, ${ }^{2}$ Icahn \\ School of Medicine at Mount Sinai / Department of Neurosurgery, New York, NY, USA, ${ }^{3}$ Mount Sinai \\ Hospital / Department of Neurosurgery, New York, NY, USA.
}

\section{Introduction:}

Fever occurs in $20-50 \%$ of critically ill neurological patients, and small temperature elevations are correlated to increased morbidity. It is therefore crucial to acutely control the temperature of such patients. Systems currently available are resource intensive and not always readily available in units, resulting in delays in treatment. EMCOOLS pads and are composed of multiple cooling units filled with graphite and water, with an adhesive underside that allows for efficient heat transfer. Pads are stored at $18^{\circ} \mathrm{C}$, and are available for immediate use.

\section{Methods:}

The retrospective analysis of the EMCOOL device included all subjects that had the device applied in the Neuroscience Intensive Care Unit (NSICU), with consistent temperature data recorded. Preliminary results were taken from 6 subjects (mean age $=53.5$, mean weight $=77.5 \mathrm{~kg}$ ), with diagnoses including subarachnoid hemorrhage (50\%), intracerebral hemorrhage $(17 \%)$, subdural hemorrhage $(17 \%)$, and pituitary tumor $(17 \%)$. All subjects were febrile $(\geq 38.0 \circ \mathrm{C})$ at initial application, $83 \%$ were administered an antipyretic within 2 hours prior and/or during the treatment period, with $50 \%$ unconscious during the treatment period. The bedside shivering assessment scale was recorded at each application.

\section{Results:}

Subjects showed a linear $1.1{ }^{\circ} \mathrm{C}\left(\right.$ T0avg $=39.0^{\circ} \mathrm{C}$, T60avg $\left.=37.9^{\circ} \mathrm{C}\right)$ drop in temperature at 60 mins from intial application, with normothermia $\left(\leq 38.0^{\circ} \mathrm{C}\right)$ achieved at 55 mins. Unconscious patients displayed a much higher rate of cooling at T60 as compared to conscious subjects ( $\triangle$ Tunconscious $=1.4$ ${ }^{\circ} \mathrm{C}$ vs $\Delta$ Tconscious $=0.5^{\circ} \mathrm{C}$ ). Of the total subjects, $33 \%$ had shivering events upon application (BSAS 2), which subsided after approximately 5 mins. There was no observed skin damage upon removal of the device.

\section{Conclusions:}

Preliminary results show the EMCOOLS pads are an effective and safe method to control temperature elevations in neurologically critically ill patients. 


\section{Case Reports}

\section{All even numbered posters will present on Friday}

All odd numbered posters will present on Saturday

All poster sessions are in Prince George Exhibit Hall A from 5:30pm-6:30pm

\section{Disorders of Consciousness}

\begin{tabular}{|c|l|l|l|}
\hline $\begin{array}{c}\text { Poster } \\
\text { Number }\end{array}$ & First Name & Last Name & Abstract Title \\
\hline 224 & Zakraus & Mahdavi & The University of Texas Southwestern Medical Center at Dallas \\
\hline 225 & Vijayalekshmi & Nair & Low Dose Baclofen Induced Reversible EEG Changes \\
\hline
\end{tabular}

\section{Head and Spine Trauma}

\begin{tabular}{|c|l|l|l|}
\hline $\begin{array}{c}\text { Poster } \\
\text { Number }\end{array}$ & First Name & Last Name & Abstract Title \\
\hline 226 & Elizabeth & Carroll & $\begin{array}{l}\text { The Dilemma of Anticoagulation in the Face of Trauma: A Case Report } \\
\text { of Superior Sagittal Sinus Thrombosis Secondary to Severe Traumatic } \\
\text { Brain Injury. }\end{array}$ \\
\hline 227 & Asher & Albertson & $\begin{array}{l}\text { Bilateral Bow Hunter's Syndrome Mimicking a Classic Seizure } \\
\text { Semiology }\end{array}$ \\
\hline 228 & Chun Fai & Cheah & $\begin{array}{l}\text { A Case Of Takotsubo Cardiomyopathy In A Patient With Severe } \\
\text { Traumatic Brain Injury And Literature Review }\end{array}$ \\
\hline
\end{tabular}

\section{ICU Organization and Technology}

\begin{tabular}{|c|l|l|l|}
\hline $\begin{array}{c}\text { Poster } \\
\text { Number }\end{array}$ & First Name & Last Name & $\begin{array}{l}\text { Abstract Title } \\
\text { Optic Nerve Sheath Diameter (ONSD) is a reliable non-invasive method } \\
\text { for monitoring treatment of patients with high ICP }\end{array}$ \\
\hline 229 & Huy & Tran & $\begin{array}{l}\text { Use of Bedside Cerebral Blood Flow Monitor to Determine the Timing } \\
\text { of Brain Death Evaluation }\end{array}$ \\
\hline 230 & Manoj & Mittal & \multicolumn{2}{|l}{} \\
\hline
\end{tabular}

\section{Intracerebral hemorrhage}

\begin{tabular}{|c|l|l|l|}
\hline $\begin{array}{c}\text { Poster } \\
\text { Number }\end{array}$ & First Name & Last Name & Abstract Title \\
\hline 231 & March & Rucci & $\begin{array}{l}\text { Calculating Quality-Adjusted Life-Years and Expectancy (QALY and } \\
\text { QALE) for Poor Grade Young Aneurysmal Subarachnoid Hemorrhage } \\
\text { Patient. }\end{array}$ \\
\hline 232 & Sowmya & Kumble & $\begin{array}{l}\text { Physiologic Effects of Incremental Early Mobilization of A Patient With } \\
\text { Acute ICH, IVH And Dual External Ventricular Drainage }\end{array}$ \\
\hline 233 & Anand & Venkatraman & $\begin{array}{l}\text { Intracranial Hemorrhage Following Consumption of Highly-Caffeinated } \\
\text { Energy Drink } \\
\text { Spontaneous Intraparenchymal Hemorrhage After Intramuscular } \\
\text { Epinephrine }\end{array}$ \\
\hline 234 & Elena & Schmidt & \\
\hline
\end{tabular}




\section{Ischemic Stroke}

\begin{tabular}{|c|l|l|l|}
\hline $\begin{array}{c}\text { Poster } \\
\text { Number }\end{array}$ & First Name & Last Name & Abstract Title \\
\hline 235 & Julian & Macedo & $\begin{array}{l}\text { Social Media Used To Determine Last Known Normal In Ischemic } \\
\text { Stroke: A Case Report }\end{array}$ \\
\hline 236 & Kinjal & Desai & $\begin{array}{l}\text { Bilateral Recurrent Artery Of Heubner Infarction After Extensive } \\
\text { Resection Of A Large Olfactory Groove Meningioma }\end{array}$ \\
\hline 237 & Nikhil & Madan & $\begin{array}{l}\text { Angioinvasive Aspergillus Associated Stroke In An Immunocompetent } \\
\text { Host. }\end{array}$ \\
\hline
\end{tabular}

\section{Medical Issues in NeurolCU}

\begin{tabular}{|c|l|l|l|}
\hline $\begin{array}{c}\text { Poster } \\
\text { Number }\end{array}$ & First Name & Last Name & Abstract Title \\
\hline 238 & Kelly & Braun & Autoimmune ganglionopathy: a rare cause of cardiac arrest \\
\hline 239 & Syeda & Alqadri & $\begin{array}{l}\text { Vasopressin Bolus Protocol Compared to Desmopressin (DDAVP) for } \\
\text { Managing Acute, Post-operative Central Diabetes Insipidus }\end{array}$ \\
\hline 240 & Bibhukalyani & Das & $\begin{array}{l}\text { Multi drug resistant CNS tuberculosis: Medical and Surgical } \\
\text { complications- A case report }\end{array}$ \\
\hline 241 & Megan & Lange & $\begin{array}{l}\text { Termination of Super-Refractory Status Epilepticus Following Cardiac } \\
\text { Arrest and Septic Shock: A Case Series }\end{array}$ \\
\hline 242 & Eileen & Vallin & $\begin{array}{l}\text { Adverse Event from Ticagrelor in a Patient with Subarachnoid } \\
\text { Hemorrhage (SAH) }\end{array}$ \\
\hline
\end{tabular}

\section{Muscle and Nerve Disorders}

\begin{tabular}{|c|l|l|l|}
\hline $\begin{array}{c}\text { Poster } \\
\text { Number }\end{array}$ & First Name & Last Name & Abstract Title \\
\hline 243 & Stasia & Rouse & $\begin{array}{l}\text { Nivolumab induced Lambert Eaton Myasthenic Syndrome in a patient } \\
\text { with Metastatic Non-Small Cell Lung Cancer }\end{array}$ \\
\hline
\end{tabular}

\section{Other}

\begin{tabular}{|c|l|l|l|}
\hline $\begin{array}{c}\text { Poster } \\
\text { Number }\end{array}$ & First Name & Last Name & Abstract Title \\
\hline 244 & Soo & Jeong & $\begin{array}{l}\text { Cerebral Microbleeds Manifesting as Stroke Symptoms During } \\
\text { Extracorporeal Membrane Oxygenation }\end{array}$ \\
\hline 245 & Lara & Zimmermann & $\begin{array}{l}\text { Acute West Nile Virus Meningoencephalitis Diagnosed via } \\
\text { Metagenomic Deep Sequencing of Cerebrospinal Fluid in a Renal } \\
\text { Transplant Patient }\end{array}$ \\
\hline 246 & Zaid & Aljuboori & $\begin{array}{l}\text { Cerebral Phaeohyphomycosis caused by Cladophialophora bantiana - } \\
\text { A Case Report and Review of Treatment Options. }\end{array}$ \\
\hline 247 & Biplab & Saha & $\begin{array}{l}\text { Far reaching consequences of Carcinoid tumor of the middle ear } \\
\text { (CTME) - the first reported case of CTME with dural sinus thrombosis } \\
\text { and distant metastasis }\end{array}$ \\
\hline 248 & Firas & $\begin{array}{l}\text { Abdulmajeed, } \\
\text { MB. ChB }\end{array}$ & $\begin{array}{l}\text { Does Neurocritical Care Need to Improve Outreach to non-Neuro } \\
\text { Specialties? }\end{array}$ \\
\hline 249 & Sung & Cho & $\begin{array}{l}\text { Transverse Myelitis due to Coxsackie B3/B4 } \\
\text { Inadvertent Intraventricular Contrast Injection: A Case Report and } \\
\text { Systematic Review of Unintended External Ventricular Drain Injections }\end{array}$ \\
\hline 250 & Ayush & Batra & $\begin{array}{l}\text { An unusual presentation of isolated brain abscess in non-traumatic } \\
\text { convexal subarachnoid hemorrhage: A case report }\end{array}$ \\
\hline 251 & Amandeep & Dolla & $\begin{array}{l}\text { Delayed Plantar Extension with Noxious Nail Bed Stimulation in Brain } \\
\text { Death }\end{array}$ \\
\hline 252 & Jonathan & Marehbian & $\begin{array}{l}\text { Somatosensory evoked potentials as Neurmonitoring Tool in } \\
\text { NeurolCU }\end{array}$ \\
\hline 253 & $\begin{array}{l}\text { Anantha } \\
\text { Ramana }\end{array}$ & Vellipuram &
\end{tabular}




\begin{tabular}{|c|l|l|l|}
\hline \multicolumn{4}{|l|}{ Peri-Operative Management } \\
\begin{tabular}{|c|l} 
Poster \\
Number
\end{tabular} & First Name & Last Name & $\begin{array}{l}\text { Abstract Title } \\
\text { latrogenic Vessel Perforation in Neuroendovascular Interventions and } \\
\text { its Management in the Neurointensive Care Unit: Targets of therapy. }\end{array}$ \\
\hline 254 & Michelle & Nunes & $\begin{array}{l}\text { A CASE OF CEREBRAL HYPERPERFUSION SYNDROME AFTER } \\
\text { SPONTANEOUS RECANALIZATION OF CAROTID INTRA-STENT } \\
\text { THROMBOSIS. }\end{array}$ \\
\hline 255 & Michelle & Nunes & $\begin{array}{l}\text { The Triphasic Response: Water imbalance after neurosurgery : A case } \\
\text { report }\end{array}$ \\
\hline
\end{tabular}

\section{Seizures}

\begin{tabular}{|c|l|l|l|}
\hline $\begin{array}{c}\text { Poster } \\
\text { Number }\end{array}$ & First Name & Last Name & $\begin{array}{l}\text { Abstract Title } \\
\text { Ketamine for Refractory Status Epilepticus: Report of Adverse Cardiac } \\
\text { Events }\end{array}$ \\
\hline 257 & Lauren & Koffman & $\begin{array}{l}\text { CNS Post Transplant Lymphoproliferative Disorder presenting as de } \\
\text { novo Status Epilepticus: Case Report }\end{array}$ \\
\hline 258 & Avni & Kapadia & $\begin{array}{l}\text { Epilepsy Surgery for New-Onset Super-Refractory Status Epilepticus: } \\
\text { A Case Report }\end{array}$ \\
\hline 259 & Simona & Ferioli, MD & $\begin{array}{l}\text { Cortical Activation, Myoclonic Status and Multiple Subdural } \\
\text { Hematomas }\end{array}$ \\
\hline 260 & Flavio & Carvalho & $\begin{array}{l}\text { Transient neurologic events in a patient with leptomeningeal } \\
\text { metastases: Case report and literature review }\end{array}$ \\
\hline 261 & Christoph & Stretz & $\begin{array}{l}\text { Pentobarbital-induced Myocardial Stunning in Status Epilepticus } \\
\text { Requiring Extracorporeal Membrane Oxygenation }\end{array}$ \\
\hline
\end{tabular}

\section{Subarachnoid hemorrhage}

\begin{tabular}{|c|l|l|l|}
\hline $\begin{array}{c}\text { Poster } \\
\text { Number }\end{array}$ & First Name & Last Name & Abstract Title \\
\hline 263 & Jesse & Robertson & $\begin{array}{l}\text { Terson's Syndrome: A Case Report and Literature Review } \\
\text { Unintended Underfeeding in Neurocritical Care Patients from Tube } \\
\text { Feeding Interruptions: Assessing the Metabolic Impact }\end{array}$ \\
\hline 264 & Angela & Vizzini & $\begin{array}{l}\text { Veno-venous ECMO as Rescue Therapy for Severe Neurogenic } \\
\text { Pulmonary Edema due to Aneurysmal Subarachnoid Hemorrhage }\end{array}$ \\
\hline 265 & Marin & Darsie &
\end{tabular}

\section{Temperature Modulation}

\begin{tabular}{|c|l|l|l|}
\hline $\begin{array}{c}\text { Poster } \\
\text { Number }\end{array}$ & First Name & Last Name & Abstract Title \\
\hline 266 & Adam & Lewis & Rethinking Myoclonic Status Epilepticus in the Young: A Case Report \\
\hline
\end{tabular}


Poster 224

\title{
The University of Texas Southwestern Medical Center at Dallas
}

\author{
Zakraus Mahdavi, Shraddha S. Mainali, Ram R. Narayan, David D. McDonagh, Venkatesh V. Aiyagari, \\ Benjamin B. Greenberg.
}

The University of Texas Southwestern Medical Center at Dallas, Dallas, TX, USA.

\section{Introduction:}

We present a case of toxic leukoencephalopathy in a young woman taking a thermogenic dietary supplement.

\section{Methods:}

A 30 year old female with unremarkable PMH except being on a diet drug "Remuvik" presented with a 2 day history of severe headache, blurry vision, photophobia, phonophobia, nausea, vomiting and brief intermittent hand spasms. Neurological exam was notable for mild right finger-to-nose ataxia and diffuse hyperreflexia. Initial MRI demonstrated extensive bilaterally symmetric T2 hyperintensities of the corpus callosum and periventricular white matter. CSF profile was unremarkable. Labs were unremarkable except for serum sodium of $127 \mathrm{mEq} / \mathrm{L}$.

\section{Results:}

Approximately 18 hours later, patient became unresponsive with bilaterally fixed-dilated pupils and decerebrate posturing. She was intubated and $100 \mathrm{gms}$ of mannitol was emergently administered with concern for cerebral edema. IV lorazepam was also given. A stat CT head showed diffuse cerebral edema. An external ventricular drain was placed emergently and $3 \% \mathrm{NaCl}$ was started. Continuous EEG was negative for seizures. Next day she started following commands and on day 5 she was discharged home with normal neurological exam.

\section{Conclusions:}

Given her presentation and MRI findings, she was diagnosed with acute toxic leukoencephalopathy due to thermogenic diet pill "Remuvik". Patient had been taking the diet drug for 6 months and had lost 30lbs. The main ingredients in Remuvik are listed as Resveratrol, caffeine-free green tea and L - Carnitine while the remaining ingredients are unknown. Similar presentation with another diet drug "Thermatrim" has been previously reported. These products are not FDA regulated and are easily available to the general public. The acute cerebral edema with decompensation was thought to be due to hyponatremia caused by Remuvik. While the mechanism of leukoencephalopathy is not well understood and further investigation is needed, spreading awareness is the key to prevent serious adverse effects of such unregulated products. 
Poster 225

\section{Low Dose Baclofen Induced Reversible EEG Changes}

Vijayalekshmi V. Nair, Elena E. Schmidt, Julius J. Latorre.

SUNY Upstate Medical University/ Neurology, Syracuse, NY, USA.

\section{Introduction:}

Baclofen is a frequently used muscle relaxant. We report a case of, low dose Baclofen causing reversible GPED's (Generalized Periodic Epileptiform Discharges). On review of literature, Baclofen toxicity/overdose has been associated with burst suppression patterns on EEG, with one case report of Baclofen toxicity causing GPED's. To the best of our knowledge there have not been reports of low dose Baclofen induced significant EEG changes.

\section{Methods:}

Case reportThe patient is an 87 year old woman, with poor baseline functional status from advanced dementia and limb contractures, on Coumadin for old DVT/PE, with sub-therapeutic INR, was admitted with new onset seizures secondary to venous infarcts over bilateral parieto-occipital areas, due to extensive venous sinus thrombosis. She was monitored on continuous video EEG and initiated on antiepileptic medications, Vimpat and Dilantin. EEG recording initially demonstrated occasional sharp waves, maximal in the left frontal region. However, because of excessive EMG artifact caused by hypertonia, the patient was started on Baclofen $5 \mathrm{mg}$. Within 24 hours patient's mental status deteriorated and EEG recording demonstrated GPED's with periods of suppression. Due to concern for drug adverse reaction, Baclofen was discontinued. The EEG reverted to pre- baclofen pattern, while her mental status slowly improved. She was provided supportive care and ultimately discharged to a rehabilitation facility.

\section{Results:}

In this elderly dementia patient, with low seizure threshold from the acute cerebral insult, low dose of Baclofen was enough to induce encephalopathy and GPED's. The absence of any metabolic disturbances along with rapid resolution of clinical and electroencephalographic abnormalities after discontinuation of the drug supports the hypothesis that these findings may be the direct cerebral toxic effect of Baclofen.

\section{Conclusions:}

latrogenic encephalopathy with Baclofen should be considered in the differential for elderly patients with low cognitive reserve 
Poster 226

\title{
The Dilemma of Anticoagulation in the Face of Trauma: A Case Report of Superior Sagittal Sinus Thrombosis Secondary to Severe Traumatic Brain Injury.
}

\author{
Elizabeth Carroll ${ }^{1}$, Rochelle R. Sweis ${ }^{2}$, José J. Biller². \\ ${ }^{1}$ Stritch School of Medicine, Loyola University Chicago, Maywood, IL, USA, ${ }^{2}$ Department of Neurology, \\ Stritch School of Medicine, Loyola University Chicago, Maywood, IL, USA.
}

\section{Introduction:}

Superior sagittal sinus thrombosis (SSST) accounts for only $0.5-1 \%$ of all strokes, with a traumatic etiology representing an uncommon occurrence. Current guidelines advocate treating SSST with anticoagulation regardless of etiology, though efficacy is controversial and not yet studied in the traumatic brain injury (TBI) patient population. We recognize the importance of alternate treatment modalities of post-traumatic SSST, particularly surgical alternatives, and the dilemmas faced with anticoagulation therapy in the trauma population.

\section{Methods:}

Case Report

\section{Results:}

We report a case of a 22-year-old male admitted with SSST who suffered severe TBI secondary to a pedestrian versus automobile collision. Imaging demonstrated bifrontal and right temporal lobe hemorrhagic contusions, scattered subarachnoid hemorrhage, diffuse cerebral edema, multiple nondepressed skull fractures, and SSST. On post trauma day two, the patient clinically deteriorated; an external ventricular device (EVD) was placed, and therapeutic heparin drip was started, despite the presence of intracranial hemorrhage and risk of EVD-related hemorrhage. The patient developed refractory elevated intracranial pressure (ICP) mandating initiation of pentobarbital to achieve burst suppression on continuous electroencephalography (CEEG) and serial administration of $23.4 \%$ hypertonic bolus and mannitol for two weeks. Hemicraniectomy and endovascular treatment were entertained though not pursued due to anticipated complications associated with concomitant anticoagulation therapy. Anticoagulation was briefly interrupted for EVD removal on post trauma day 17. He was extubated on post trauma day 20 and transitioned to warfarin. Repeat imaging showed complete recanalization of the superior sagittal sinus. The patient was discharged to inpatient rehabilitation after a 28-day hospital course.

\section{Conclusions:}

Management of SSST secondary to TBI remains controversial as these patients present with multiple confounding factors, further complicated by the lack of treatment guidelines. Further studies are needed to determine which independent or combined medical and surgical treatment modalities will decrease morbidity and mortality in this patient population. 
Poster 227

\section{Bilateral Bow Hunter's Syndrome Mimicking a Classic Seizure Semiology}

Asher J. Albertson, Terrance T. Kummer.

Washington University/Department of Neurology, St. Louis, MO, USA.

\section{Introduction:}

Rotational vertebrobasilar insufficiency, also called Bow Hunter's syndrome after the symptom-inducing head position adopted when aiming a bow, is a rare cause of posterior circulation ischemia. We present a case of an 84-year-old woman who presented to Barnes-Jewish Hospital with several days of episodic vertigo and gait instability. Two weeks prior to presentation she had fallen and struck her head. Imaging revealed a complex $\mathrm{C} 1$ Fracture as well as an odontoid fracture with posterior displacement. She began having positional spells characterized by loss of consciousness, gaze deviation, fencer posturing, and sonorous breathing.

\section{Methods:}

Review of clinical records and literature review.

\section{Results:}

The spells were initially highly concerning for seizures. The patient was monitored on continuous video EEG, however no seizures were detected during typical spells. A CT angiogram revealed an occluded right vertebral artery at the level of $\mathrm{C} 2$ with diminutive vs. absent posterior communicating arteries isolating the posterior circulation. Subsequent MR angiography revealed a patent right vertebral artery with no evidence of stroke. Catheter cerebral angiography demonstrated a patent left vertebral artery. Turning the head 15 degrees during the procedure, however, elicited a typical spell and completely occluded the left vertebral artery. The patient underwent occipitocervical fusion, but unfortunately suffered a multifocal posterior circulation stroke and was discharged with hospice care.

\section{Conclusions:}

We present an unusual case of rotational vertebrobasilar insufficiency that mimicked a classic sezure semiology and presented several diagnostic dilemmas in the ICU. In this case, traumatic injury resulted in likely bilateral positional vertebral artery occlusion with resultantly profound brainstem ischemia. Bow hunter's syndrome should be considered in all cases of positional neurological spells, particularly in the setting of neck injury. 
Poster 228

\title{
A Case Of Takotsubo Cardiomyopathy In A Patient With Severe Traumatic Brain Injury And Literature Review
}

\author{
Chun Fai Cheah ${ }^{1}$, Mario M. Kofler ${ }^{2}$, Alois A. Schiefecker ${ }^{2}$, Ronny R. Beer ${ }^{2}$, Gert G. Klug ${ }^{3}$, Paul P. \\ Rhomberg $^{4}$, Bettina B. Pfausler ${ }^{2}$, Erich E. Schmutzhard ${ }^{2}$, Raimund R. Helbok'.
}

1. Department of Neurology, Medical University of Innsbruck 2. Dept. of Neurology, PGH, 1. Innsbruck 2. Penang, Malaysia, Austria, ${ }^{2}$ Department of Neurology, Medical University of Innsbruck, Innsbruck, Austria, ${ }^{3}$ University Clinic of Internal Medicine III, Medical University of Innsbruck, Innsbruck, Austria, ${ }^{4}$ Department of Neuroradiology, Medical University of Innsbruck, Innsbruck, Austria.

\section{Introduction:}

Takotsubo cardiomyopathy (TC) is known to occur in patients with subarachnoid haemorrhage (SAH) but is rarely reported in patients with traumatic brain injury (TBI). Here we present a TBI patient with complicated clinical course developing severe TC and compared to previously published reports.

\section{Methods:}

Case report and literature review.

\section{Results:}

A 73 years-old-woman was admitted to our tertiary care hospital because of TBI with admission Glasgow Coma Scale score of 14 . Computed tomography (CT) scanning of the brain revealed an acute subdural hematoma and traumatic SAH over left hemisphere and a small left frontal hemorrhagic contusion. Six hours later she deteriorated and head-CT showed significant progression of right frontal hemorrhage with intraventricular expansion and a midline shift. Hematoma evacuation was immediately performed.

Postoperatively the patient developed cardiogenic shock necessitating an increasing dose of noradrenaline, neosynephrine and dobutamine to achieve a cerebral perfusion pressure of $>65 \mathrm{mmHg}$. Echocardiography demonstrated severe left ventricular myocardial dysfunction suggestive for TC, supported by raised troponin-T and NT-proBNP levels and abnormalities in ECG. Continuous infusion of levosimendan was added and maintained for 28 hours which led to stabilization allowing noradrenalin to be decreased the following days. Repeated echocardiography 8 days later showed normalized cardiac function. The patient's condition gradually improved and was extubated after 11 days fully awake with mild left facial-brachial weakness.

\section{Conclusions:}

Here we present a complicated case of TC with TBI developing cardiogenic shock within 24 hours of admission. We will compare the patient's TC characteristics and clinical course with published cases $(\mathrm{N}=$ 16) of TC with TBI. Further studies of TC in patients with TBI and the utility of levosimendan is warranted. 


\section{Poster 229}

\section{Optic Nerve Sheath Diameter (ONSD) is a reliable non-invasive method for monitoring treatment of patients with high ICP}

Huy $\operatorname{Tran}^{1}$, Robert R. Alunday ${ }^{2}$, Juan Carlos J. Vera ${ }^{2}$, Kyna K. Seale ${ }^{2}$, Isaac I. Tawil ${ }^{2}$, Howard H. Yonas ${ }^{2}$.

${ }^{1}$ University of New Mexico Hospital / Department of Neurosurgery, Albuquerque, NM, USA, ${ }^{2}$ University of New Mexico / Department of Neurosurgery, Albuquerque, NM, USA.

\section{Introduction:}

Optic nerve sheath diameter (ONSD) measurement using ultrasound has been proposed as a reliable method for non-invasive assessment of intracranial pressure (ICP). We report a case of using ONSD to monitor ICP in a TBI patient with elevated ICP undergoing medical treatment with acetazolamide. We hypothesize that a difference in ONSD could be detected with ultrasound before and after treatment.

\section{Methods:}

Patient is a 31 year old man with mild TBI due to assault. His Head CT reveals a long calvarial fracture extending along the superior sagittal suture line and posteriorly into the left parietal bone, as well as a large epidural hematoma overlying the frontoparietal vertex near midline, and causing inferior displacement and extrinsic compression of the superior sagittal sinus. His physical exam reveals a young man with right orbital ecchymosis who is sleepy but easily arousable with a GCS of 456 and no motor deficits. Dilated fundus exam by ophthalmology reveals grade 2-3 papilledema consistent with elevated ICP. The patient complains of persistent headaches and nausea that is unremitting. Acetazolamide was started to decrease ICP. We measured ONSD with a sonosite ultrasound device prior to start of acetazolamide and 2 days afterwards. Two measurements were taken on each eye, one in the horizontal and vertical orientation each.

\section{Results:}

The average ONSD was $6.4 \mathrm{~mm}$ on the right eye and $6.4 \mathrm{~mm}$ on the left eye prior to initiation of treatment. On the day after treatment ONSD was $4.65 \mathrm{~mm}$ on the right and $5.1 \mathrm{~mm}$ on the left eye. The patient's headache improved and nausea resolved. The next day ONSD was $3.5 \mathrm{~mm}$ on the right and $3.6 \mathrm{~mm}$ on the left eye. Headache and nausea completely resolved.

\section{Conclusions:}

This case report affirms that ultrasound measurement of ONSD could be used reliably to assess ICP noninvasively during the course of treatment for elevated ICP. 
Poster 230

\title{
Use of Bedside Cerebral Blood Flow Monitor to Determine the Timing of Brain Death Evaluation
}

\author{
Manoj K. Mittal.
}

Kansas University Medical Center/ Neurology, Kansas City, KS, USA.

\section{Introduction:}

Timing of brain death evaluation could be crucial in maintaining organ perfusion for donation. A new bedside cerebral blood flow monitor (cFLOW monitor from Ornim) has not been previously studied for determining the timing of brain death examination.

\section{Methods:}

We present here a case illustrating the role of bedside blood flow monitoring in determining the timing of brain death evaluation.

\section{Results:}

A 73 year-old-woman presented with acute right middle cerebral artery stroke and bilateral internal carotid artery occlusions. She was not a candidate for intravenous thrombolysis or endovascular therapy due to unknown time of symptoms onset. Her initial NIHSS was 19 (right gaze deviation, mild aphasia, mild dysarthria, left facial droop, left hemiparesis, left sided decreased sensation and neglect). Day-2, she got intubated for hypoxic respiratory failure. Day-3, CT head showed cerebral edema with midline shift of 8 $\mathrm{mm}$. Patient was not a decompressive hemicraniectomy candidate. Day-5, patient was comatose. Day-7, patient lost bilateral pupillary reflex. CT head showed worsening midline shift of $17 \mathrm{~mm}$ with right uncal herniation, bilateral anterior cerebral artery and left posterior cerebral artery stroke, and brainstem compression. Day-9, bedside cerebral blood blood flow monitoring was started with right sided cerebral blood flow index (CFI) of 16 and left side CFI of 35. Patient met criteria for brain death except that she was still breathing over the ventilator. Patient was extubated for comfort measures. After 60 minutes patients stopped breathing. Her CFI dropped $<10$ bilaterally. Patient underwent cardiac arrest after 10 minutes and then both $\mathrm{CFI}$ were $<8$. Patient was not a candidate for organ donation.

\section{Conclusions:}

Bedside cerebral blood flow monitoring may assist in determining the timing of brain death evaluation in comatose patients with imminent brain death. Patients with $\mathrm{CFI}<10$ may be considered for brain death evaluation. Our finding needs further confirmation. 
Poster 231

\title{
Calculating Quality-Adjusted Life-Years and Expectancy (QALY and QALE) for Poor Grade Young Aneurysmal Subarachnoid Hemorrhage Patient.
}

\author{
March Rucci ${ }^{1},{ }^{2}$, William W. Freeman ${ }^{3}$, Sarah S. Peacock ${ }^{3}$. \\ ${ }^{1}$ Mayo Clinic / Center for the Science of Health Care Delivery, Jacksonville, FL, USA, ${ }^{2}[[[N O T$ FOUND]]] , \\ ${ }^{3}$ Mayo Clinic / Neurology, Jacksonville, FL, USA.
}

\section{Introduction:}

ICUs are high cost in the U.S., comprising about $17 \%$ of the US GDP. Pressure is being placed on hospitals and intensivists to reduce costs, including earlier palliative care engagement to shorten length of stay.. As the U.S. migrates to a value-based system, further pressure will be made on reducing prolonged and expensive ICU interventions, similar to quality adjusted life year (QALY) cutoff values to justify costs.

\section{Methods:}

A young 23 year old man presented with worst headache of his life, and was found comatose by EMS and referred to our NeurolCU. He had a $2.2 \mathrm{~cm}$ giant basilar aSAH with intraventricular hemorrhage. The aneurysm was coiled endovascularly with external ventricular drain placed. He required therapeutic hypothermia, osmotherapy, induced hypertension and balloon angioplasty and intraarterial verapamil for refractory basilar and bilateral middle cerebral artery vasospasm. He had refractory intracranial pressure from global cerebral edema and around post-operative day \#13 required bifrontal craniectomy. Later percutaneous tracheostomy, PEG tube, and ventriculoperitoneal shunting were performed. His total costs exceeded $\$ 300,000$ U.S. dollars.

\section{Results:}

One year later, his modified Rankin scale was zero, and he went to college. His QALY (quality adjusted life year) for the rest of his year was 0.833 given a utility of 1 . His physicians felt he should live to a normal life expectancy of 77 years of age, $Q$ is quality of life weight $=1$ (perfect health, utility $=1$ ), L is residual life expectancy $=54$ more years. His QAL-Expectancy 2,3 is about 54 life-years gained which divided over his life span is about $\$ 5,555 /$ year and less than the current CMS reported value of $\$ 9,500$ per year.

\section{Conclusions:}

This case exemplifies how high cost care can be delivered to deliver cost-effective, high quality care and underscore the need for integrated high-complexity neurolCU care. 
Poster 232

\title{
Physiologic Effects of Incremental Early Mobilization of A Patient With Acute ICH, IVH And Dual External Ventricular Drainage
}

\author{
Sowmya Kumble ${ }^{1}$, Mackenzie M. Burch ${ }^{1}$, Elizabeth E. Zink ${ }^{2}$, Mona M. Bahouth ${ }^{3}$, Robert R. Stevens ${ }^{4}$ \\ ${ }^{1}$ Johns Hopkins Hospital/Department of Physical Medicine \& Rehabilitation, Baltimore, MD, USA, ${ }^{2}$ Johns \\ Hopkins Hospital/Department of Neuroscience Nursing, Baltimore, MD, USA, ${ }^{3}$ Johns Hopkins \\ SOM/Department of Neurology, Baltimore, MD, USA, ${ }^{4}$ Johns Hopkins SOMI/Johns Hopkins Neuroscience \\ Critical Care, Baltimore, MD, USA.
}

\section{Introduction:}

Early mobility in the intensive care unit setting is associated with a number of positive effects including improved quality of life. Though there is a strong body of evidence supporting early mobility in medical intensive care units, the benefits of very early mobilization after acute stroke are yet unclear as early hemodynamic variability in patients with impaired cerebral auto regulation is of concern. Another potential barrier to early mobilization is the presence of an external ventricular drain (EVD) for cerebrospinal fluid diversion and intracranial pressure (ICP) monitoring. This case demonstrates hemodynamic and ICP responses to progressive, device assisted mobility interventions during the acute phase of intracerebral hemorrhage $(\mathrm{ICH})$ in the setting of persistent elevations of ICP requiring two EVDs.

\section{Methods:}

A 55 year-old man was admitted to the Neuroscience Critical Care Unit with an acute thalamic ICH and intraventricular hemorrhage requiring placement of two EVDs. Starting on day 3 following $\mathrm{ICH}$ onset, the patient underwent progressive mobilization following the Johns Hopkins NCCU Activity and Mobility Algorithm. Range of motion exercises were performed initially, progressing to supine cycle ergometry followed by incremental verticalization using the tilt table (Sara Combilizer®Arjo Huntleigh Inc., II). Blood pressure, heart rate, oxygen saturation, and ICP were recorded before, during and after the mobility interventions.

\section{Results:}

No adverse neurologic effects were noted during these mobility interventions. Recorded hemodynamic variables and ICP remained within the set goals throughout. Moreover the patient was able to tolerate 45 degrees of verticalization on the tilt table.

\section{Conclusions:}

Progressive, device assisted early mobilization was feasible when titrated by skilled healthcare professionals in a critically ill hemorrhagic stroke patient with EVDs. Studies on larger patient samples are needed to improve our understanding of the hemodynamic and neurophysiologic responses to establish safety of progressive early mobilization of critically ill patients with acute stroke. 
Poster 233

\section{Intracranial Hemorrhage Following Consumption of Highly-Caffeinated Energy Drink}

Anand Venkatraman, Ayaz A. Khawaja, Angela A. Shapshak.

University of Alabama at Birmingham / Department of Neurology, Birmingham, AL, USA.

\section{Introduction:}

We describe a case of a patient with uncontrolled hypertension (HTN) and prior intracranial hemorrhage (ICH) who developed an intracranial hemorrhage shortly after consuming Redline, a heavily-caffeinated energy drink.

\section{Methods:}

A 57-year old Caucasian male with prior history of $\mathrm{ICH}$ and chronic untreated $\mathrm{HTN}$ was transferred to our service for evaluation of $1.8 \times 1.3 \mathrm{~cm} \mathrm{ICH}$ in the left thalamus. Blood pressure had been elevated in the Emergency room there and he had been started on a nicardipine infusion. $\mathrm{NIH}$ Stroke scale was 1 . ICH score was 0 . Admission labs were normal. Urine drug screen was negative. On questioning, patient revealed that symptoms had started within 2 hours of consumption of 1 bottle of Redline, an energy drink. He was not a regular user but did consume it whenever he needed to get a lot of work done. MRI of the brain did not show any vascular malformation or other lesion. Multiple remote hemorrhages were seen in the subcortical areas. We stared lisinopril and weaned off his nicardipine infusion. He was discharged with minimal deficits.

\section{Results:}

The high caffeine content is the most likely component of the drink that led to the $\mathrm{ICH}$, given that high caffeine consumption is linked to increased risk of hemorrhagic strokes. Caffeine also has effects on platelet aggregation and endothelial function that could raise stroke risk. Generalizability is limited by the fact that the patient had uncontrolled HTN and prior ICHs. However, given that $15 \%$ of adults in the USA have uncontrolled $\mathrm{HTN}$, and given that $\mathrm{ICH}$ account for $10-15 \%$ of all strokes, a large population is at risk.

\section{Conclusions:}

To our knowledge this is the first report of intracranial hemorrhage following consumption of an energy drink. Consumers must exercise caution, especially in the setting of uncontrolled risk factors. 


\title{
Poster 234
}

\section{Spontaneous Intraparenchymal Hemorrhage After Intramuscular Epinephrine}

\author{
Elena Schmidt, Varada V. Nair, Gene G. Latorre.
}

SUNY Upstate University Hospital / Department of Neurology, Syracuse, NY, USA.

\section{Introduction:}

Often times medications given in emergency cases have unintended consequences, sometimes posing even more harm than the reason for their administration. We report a case of a young lady with history of anaphylactic reaction who received i.m. epinephrine after developing allergic reaction to antibiotic, resulting in bilateral intracerebral hemorrhage $(\mathrm{ICH})$.

\section{Methods:}

Our case is of a 42 year old female with history of anaphylactic reaction who had been recently started on Cephalexin for orbital cellulitis. She was sent to ED after having a syncopal event in the doctor's office. While in ED, the patient was administered $0.3 \mathrm{mg}$ of 1:1,000 Epinephrine i.m. because of suspected anaphylactic reaction. Shortly after, she complained of nausea, vomiting and developed right sided weakness and numbness. Immediate CT head revealed two areas of $\mathrm{ICH}$, within the left parietal and right occipital lobes. Extensive work-up ensued, with CTA head and neck (negative for vessel anomalies), cerebral DSA (negative for vasculitis), MRI brain w/wo contrast (negative for malignancy or amyloid angiopathy), CT thorax and abdomen (negative for malignancy). Serum studies for vasculitis work-up were also unrevealing. Echocardiogram did not show evidence for chronic hypertension such as LV hypertrophy.

\section{Results:}

Although there have been reports in the literature of spontaneous intracranial hemorrhage (intraparenchymal or subarachnoid) after various ways of epinephrine administration, in our patient's case, the extensive work-up done to exclude other etiologies stands out. This strengthens the hypothesis that epinephrine, causing an acute spike in blood pressure, ultimately led to spontaneous ICH. In addition, the case of our patient is unique in that she developed two areas of $\mathrm{ICH}$, in a location typical for Posterior Reversible Encephalopathy Syndrome (PRES), a syndrome known to be caused by significant elevation in blood pressures, suggesting a common underlying pathophysiology.

\section{Conclusions:}

Careful consideration of indications should occur before administering such potentially harmful treatments. 
Poster 235

\title{
Social Media Used To Determine Last Known Normal In Ischemic Stroke: A Case Report
}

\author{
Julian Macedo ${ }^{1}$, Brandon B. Foreman ${ }^{2}$, Dawn D. Kleindorfer ${ }^{2}, 3$. \\ ${ }^{1}$ University of Cincinnati, Neurocritical Care/Vascular Neurology, Cincinnati, OH, USA, ${ }^{2}$ University of \\ Cincinnati, Cincinnati, USA, ${ }^{3}[[[N O T$ FOUND]]].
}

\section{Introduction:}

"Last known normal" (LKN) time remains the standard for determining the onset of acute ischemic stroke and appropriateness of providing acute therapies. As older adults become more familiar with social media platforms, these applications may become a source of recognizing when a patient was LKN.

\section{Methods:}

\section{Case Report}

\section{Results:}

We report an 82 year-old woman who was "found down" at home. The patient lived independently, and was able to crawl to a telephone for help. On arrival to the emergency department, she had a right middle cerebral artery syndrome with an NIH stroke scale of 17 . She had a decreased level of arousal and severe dysarthria which precluded assessment of her LKN. The patient's son reported that he had last seen her normal 9 hours prior, placing her outside the time window for acute therapies. However, the patient's granddaughter reported that the patient had been logged into Facebook $<1$ hour prior to her admission "chatting" and commenting on photos. "Timestamps" of comments left on photos by the patient provided exact times of the patient's activity. The only logical means of being able to perform these relatively high-functioning tasks would have been if the she was normal at the time of posting, thereby establishing her LKN. The patient was treated with systemic t-PA followed by endovascular therapy for a proximal M1 occlusion. The patient had rapid improvement of her stroke symptoms. She was discharged home with an $\mathrm{NIH}$ stroke scale of 2.

\section{Conclusions:}

This patient's recent use of the social media was critical in determining the patient's LKN, leading to lifesaving acute stroke therapy. Providers should be aware that social media may serve as a useful source of symptom onset information. In this case, it led to good outcome and discharge home. 


\title{
Poster 236
}

\section{Bilateral Recurrent Artery Of Heubner Infarction After Extensive Resection Of A Large Olfactory Groove Meningioma}

\author{
Kinjal A. Desai', Lauren L. Spiegel', Ranier R. Reyes ${ }^{2}$, Aditya A. Vedantam ${ }^{3}$, Rahul R. Damani ${ }^{1}$, Eric E. \\ Bershad ${ }^{1}$.
}

\author{
${ }^{1}$ Baylor College of Medicine, Division of Neurocritical Care, Houston, TX, USA, ${ }^{2}$ Baylor College of \\ Medicine, Department of Neurology, Houston, TX, USA, ${ }^{3}$ Baylor College of Medicine, Department of \\ Neurosurgery, Houston, TX, USA.
}

\section{Introduction:}

Bilateral Recurrent Artery of Heubner (RAH) infarctions have been seldomnly reported in the literature. Even more so for those cases that have occurred subsequent to Neurosurgical extensive resections of large invasive Olfactory Groove Meningioma. RAH, a branch of the anterio-inferior cerebral artery, supplies anterior limb of the internal capsule, anterior caudate, putamen and globus pallidus. Infarction typically results in contralateral paresis of the arm and face. Other symptoms can occur i.e. choreiform movements, abulia, attention disorder, impaired memory, apathy, decreased spontaneity, depression, dementia etc. We present a case of Bilateral RAH infarcts as a complication of a large Olfactory Groove Meningioma resection.

\section{Methods:}

We did an extensive chart review of our patient during post-operative Neurointensive Care unit stay, rest of the hospital stay and discharge follow up at 1 month.

\section{Results:}

Our patients Brain MRI done as a part of routine post-operative imaging showed bilateral caudate head infarcts in the territory of RAH. Post-operative exam was significant for a left hemianopsia and right super quadrantopsia with color desaturation. Patient did not experience any new weakness or movement related problems. He did have changes in cognition (forgetfulness \& Irritability) along with a subjective loss of sense of smell but these were consistent with his pre-op assessment. Olfactory Groove Meningioma's comprise $10 \%$ of all intracranial meningiomas, are slow growing and tend to engulf and compress neighboring structures. Most common complications of Olfactory Groove Meningioma resections are post-operative cerebral edema, CSF leak, seizures, CNS infections, hydrocephalus and rarely brain ischemia.

\section{Conclusions:}

Bilateral RAH infarction, although rare has been reported in literature in association with vascular anomalies and other stroke risk factors. Cerebral infarction involving the ACA territories remains a known adverse complication of large olfactory groove meningioma resections, but bilateral infarcts due to these have not been reported before. 
Poster 237

\title{
Angioinvasive Aspergillus Associated Stroke In An Immunocompetent Host.
}

\author{
Nikhil Madan', Tapan T. Kavi', Tamara T. Majic ${ }^{1}$, Shouri S. Lahiri' ${ }^{1}$ \\ ${ }^{1}$ Cedars-Sinai Medical Center/ Neurocritical care, Los Angeles, CA, USA, ${ }^{2}$ Cedars-Sinai Medical Center / \\ Neurocritical Care, Los Angeles, CA, USA.
}

\section{Introduction:}

Aspergillus vasculitis is an under-recognized cause of stroke in immunocompetent hosts, especially when other risk factors are present. We present a case of autopsy proven angioinvasive aspergillus causing strokes in an immunocompetent host, and review the characteristic imaging findings to aid diagnosis.

\section{Methods:}

59-year-old female developed cardiogenic shock after three-vessel-Coronary artery bypass grafting (CABG) using saphenous vein grafts requiring intra-aortic balloon pump placement. This was complicated by aortic dissection, and she underwent replacement of the ascending aortic arch. Refractory cardiogenic shock ensued for which she underwent placement of veno-arterial extracorporeal membrane oxygenation. Postoperatively, she was noted to be in coma, and a non-contrast CT of the brain showed small multiple small ischemic strokes bilaterally. With persistent multi-organ failure, she was ultimately transitioned to comfort care and passed. Autopsy revealed multiple perivascular petechial hemorrhagic infarcts involving white matter, deep gray matter and cerebellum on gross specimen. Histopatholgic study showed Aspergillus associated acute and chronic inflammation of blood vessel, and surrounding gliosis. Aspergillus was also found in coronary grafts and kidneys.

\section{Results:}

Aspergillus associated cerebral vasculitis was considered less likely, as CABG, Extracorporeal membrane oxygenation (ECMO) device-related thrombosis and acute $\mathrm{MI}$ were the leading differentials for stroke here, and no obvious immunosuppression was evident. Cerebral aspergillosis can occur from direct spread from sinus infections or through hematogenous mode, and seemed to have originated from coronary grafts in this case.

\section{Conclusions:}

Aspergillus has a predilection for posterior circulation arteries, and lacunar-type infarcts or petechial hemorrhages within the midbrain, thalami, or corpus callosum are characteristic. These findings should raise suspicion for aspergillus, especially without objective evidence of other mechanisms of stroke. Early initiation of anti-fungal therapy may improve the likelihood of survival, and confirmatory testing in the form of blood vessel imaging, CSF analysis and fungal blood cultures should be performed in suspected cases. 
Poster 238

\title{
Autoimmune ganglionopathy: a rare cause of cardiac arrest
}

\author{
Kelly Braun.
}

Neurological Institute, Cleveland Clinic, Cleveland, OH, USA.

\section{Introduction:}

Autoimmune autonomic ganglionopathy is a rare disorder characterized by pandysautonomia that occurs as a result of autoantibodies to ganglionic nicotinic acetylcholine receptors.

\section{Methods:}

We describe a 27 year old male with autoimmune ganglionopathy previously treated with IVIG who suffered cardiac arrest and anoxic brain injury as a complication of this disorder.

\section{Results:}

The patient had a history of multiple autoimmune diseases (DM1, autoimmune hepatitis, Hashimoto's thyroiditis, celiac disease, antiphospholipid syndrome and ulcerative colitis). To alleviate his pre-syncopal lightheadedness related to dysautonomia, he would typically kneel and place his head on his folded arms. The patient was found unresponsive in this position in PEA arrest. He underwent CPR followed by therapeutic hypothermia $\left(34^{\circ} \mathrm{C} \times 24 \mathrm{~h}\right)$. The initial exam off sedation showed an obtunded patient with intact pupillary and corneal reflexes, but no tracking or command following. Though he moved all extremities spontaneously, the movements were not purposeful and had a choreiform quality. Notable labs were an elevated AChR ganglionic neuronal antibody $(5.44 \mathrm{nmol} / \mathrm{L}$ on hospital day 8 and 3.10 $\mathrm{nmol} / \mathrm{L}$ on hospital day 15 ; normal $<0.02 \mathrm{nmol} / \mathrm{L}$ ). MRI brain showed symmetric diffusion restriction and FLAIR changes throughout the brainstem, thalami and cerebellum, however there was no cortical diffusion restriction. EEG showed generalized intermittent rhythmic slowing, which was maximal bifrontally. He was treated with Methylprednisolone $30 \mathrm{mg}$ daily for 23 days and transitioned to Prednisone $40 \mathrm{mg}$ daily. At the time of discharge to an acute rehabilitation facility, the patient followed most simple commands and moved all of his extremities against resistance, though he was noted to have diffuse hypotonia.

\section{Conclusions:}

To our knowledge, this is the first reported case of cardiac arrest attributed to autoimmune autonomic ganglionopathy. While rare, this is a serious complication of this syndrome. 
Poster 239

\title{
Vasopressin Bolus Protocol Compared to Desmopressin (DDAVP) for Managing Acute, Post- operative Central Diabetes Insipidus
}

\author{
Syeda L. Alqadri ${ }^{1}$, Christopher C. Newey, $M D^{2}$, Premkumar P. Nattanmai, MD ${ }^{3}$, Robert R. Bell, PA-C , \\ Ashley A. Ausmus 5 .
}

${ }^{1}$ University of Missouri/Department of Neurology, Columbia, MO, USA, ${ }^{2}$ University of Missouri/Department of Neurology and Department of Neurosurgery., Columbia, MO, USA, ${ }^{3}$ University of Missouri/Department of Neurology and Department of Neurosurgery, Columbia, MO, USA, ${ }^{4}$ University of Missouri/Department of Neurosurgery, Columbia, MO, USA, ${ }^{5}$ University of Missouri/Department of Pharmacology, Columbia, MO, USA.

\section{Introduction:}

Management of post-operative central diabetes insipidus (DI) is focused on replacing urine output with free water. This may not always be sufficient, and desmopressin (DDAVP) is needed. The use of DDAVP, however, is known to cause profound changes in sodium particularly if the triphasic response postpituitary surgery is occurring. Herein, we report a case using a dilute vasopressin bolus protocol in managing hypovolemia in acute, post-operative, central DI.

\section{Methods:}

Case report comparing two protocols for DI management. Statistical analysis was performed on serum sodium, urine specific gravity, and urine output using student t-test. $\mathrm{P}<0.05$ was considered significant.

\section{Results:}

Patient is a 16-year-old male admitted to the neurosciences intensive care unit (NSICU) after bifrontal decompressive craniotomy for severe traumatic brain injury from self-inflicted gunshot wound. Immediately post-operatively, he developed increased urine output. He arrived to NSICU hypovolemic (3.4 liters negative in three hours) and hypernatremic $(148 \mathrm{meq} / \mathrm{L})$. He was resuscitated with intravenous fluids including a dilute vasopressin bolus protocol. This protocol consisted of 1 unit of vasopressin in 1 liter of $0.45 \%$ normal saline. This was given in boluses based on the formula: urine output minus one hundred. At the start of this protocol, the serum sodium was 148 and one-hour urine output was 1 liter. This protocol was continued for 48 hours. Endocrinology was consulted and recommended changing to DDAVP. Serum sodium was 149[NP1] and one-hour urine output $320 \mathrm{cc}$ prior to first dose of DDAVP. Comparing the bolus protocol to the DDAVP protocol, the average sodium was $143.8+3.2$ and $149.6+3.2$ $\mathrm{meq} / \mathrm{L}(\mathrm{p}=0.0001)$, average urine output was $433.2+354.4$ and $422.3+276.0 \mathrm{cc} / \mathrm{hr}(\mathrm{p}=0.90)$, and average specific gravity was $1.019+0.009$ and $1.016+0.01$, respectively $(p=0.42)$.

\section{Conclusions:}

A protocol using dilute vasopressin bolus is an alternative for managing acute, central DI postoperatively, particularly with acute hypovolemia. Additional studies are needed to address its efficacy in a larger sample. 
Poster 240

\title{
Multi drug resistant CNS tuberculosis: Medical and Surgical complications- A case report
}

\author{
Bibhukalyani Das, Shantanu S. Shubham. \\ Institute of Neurosciences Kolkata, Kolkata, India.
}

\section{Introduction:}

Global burden of tuberculosis is still high particularly in developing world. India is the largest TB burden country accounting for $1 / 5$ th of the global incidence.CNS tuberculosis is the most severe form of infection with Microbacterium tuberculosis. Emergence of MDR(multi drug resistant) tuberculosis has compounded the risk and adverse outcome. Fatality rate of MDR TB meningitis is $57 \%$ with significant functional impairment in most of the survivors. Mortality $>90 \%$ if patient is HIV positive.

\section{Methods:}

We report a case of $15 \mathrm{yrs}$ old girl from Eastern India case of MDR - CNS tuberculosis with a protracted clinical course of 2 years. She developed a whole range of complications including Hydrocephalus, optochiasmatic arachnoiditis with secondary optic atrophy, multiple tuberculomas, cerebellar and brainstem tubercular abscesses and SIADH with Hyponatraemia. Our case is notable for few rare complications in the form of Transverse Sinus Thrombosis secondary to chronic meningitis necessitating oral anticoagulation. The patient also developed various side effects of long term ATT such as -(i) ATT induced Hepatitis. (ii) Moxifloxacin induced seizures and re-adjustment of antiepileptics due to interaction with antitubercular drugs. She was managed with 9 antitubercular drugs ( 5 first line drugs) along with second line drugs (Amikacin, Levofloxacin, Cycloserine, Ethionamide) Her clinical course was complicated by obstructive hydrocephalus requiring EVD, VP Shunt and Shunt Revision . Developed acute SDH possibly secondary to shunt and required surgical drainage . Later she developed posterior fossa tubercular abscess and needed craniectomy. Pus from tubercular abscess grew MTB resistant to Rifampicin and Isoniazid. So the patient was maintained on second line drugs, ultimately succumbed to hospital acquired pneumonia.

\section{Results:}

NA

\section{Conclusions:}

CNS tuberculosis if associated with multiple medical, surgical complications, impose real critical care challenges compounded by MDR which often encountered in a developing country like India. 
Poster 241

\section{Termination of Super-Refractory Status Epilepticus Following Cardiac Arrest and Septic Shock: A Case Series}

Megan Lange, Rebecca R. Horrell.

University of Maryland Medical Center, Neurocritical Care Unit, Baltimore, MD, USA.

\section{Introduction:}

Super-refractory status epilepticus, defined as seizures persisting despite anesthetics, is associated with high morbidity and mortality.

\section{Methods:}

Here we present two cases of super-refractory status epilepticus intractable to aggressive therapies, including but not limited to anesthetics, electroconvulsive therapy, and immunotherapy.

\section{Results:}

In both cases, the patients developed sepsis and cardiac arrest following prolonged hospitalizations with subsequent termination of seizure activity and improvement in electroencephalogram findings and neurologic exams.

\section{Conclusions:}

A review of the literature revealed a variety of publications describing super-refractory status epilepticus as a result of sepsis or cardiac arrest, but there is limited data describing either complication as therapeutic for status epilepticus. We propose that the systemic effects associated with profound sepsis, or the brief electrographic silence occurring in the setting of cardiac arrest could have played a role in halting seizures in these patients. We describe two theories regarding the potential mechanism by which cardiac arrest or sepsis could play a role in termination of seizures. Exploration into specific mediators involved in these conditions and their relationship to status epilepticus could uncover therapeutic targets. Targeted therapies could demonstrate promise in effectively treating super-refractory status epilepticus, thereby improving morbidity and mortality rates. 


\title{
Poster 242
}

\section{Adverse Event from Ticagrelor in a Patient with Subarachnoid Hemorrhage (SAH)}

\author{
Eileen C. Vallin ${ }^{1}$, William W. Knight IV, MD, FACEP, FNCS ${ }^{2}$, Shaun S. Keegan, Pharm. D., BCPS ${ }^{3}$.
}

${ }^{1}$ University of Cincinnati, Dept. of Neurology and Rehabilitation Medicine, Cincinnati, OH, USA, ${ }^{2}$ University of Cincinnati, Division of Neurocritical Care, Cincinnati, OH, USA, ${ }^{3}$ UC Health-University of Cincinnati Medical Center, Cincinnati, OH, USA.

\section{Introduction:}

Ticagrelor is approved for prevention of cardiovascular events in adults with acute coronary syndrome (ACS)1. We present a patient with SAH who developed thrombus during coiling procedure that was treated with abciximab followed by ticagrelor and aspirin, with potentially devastating consequences.

\section{Methods:}

A 64-year-old male presented after sudden onset severe headache. Imaging revealed SAH from a ruptured basilar tip aneurysm. The patient was neurologically intact with mild confusion, but declined during transfer and required intubation. An external ventricular drain was placed for hydrocephalus. During cerebral angiogram with coil embolization, a thrombus formed on the coil. Intra-arterial abciximab was used with resolution of thrombus. He was extubated post procedure. Aspirin and ticagrelor (90 mg twice daily) were prescribed. The following day, the patient became increasingly lethargic with an increased respiratory rate (30s). He reported no perception of increased work of breathing. Portable chest radiograph demonstrated only mild pulmonary edema. He did not have an oxygen requirement. Venous blood gas demonstrated a pH 7.52 with a pCO2 of 27 , suggesting a respiratory alkalosis. Transcranial dopplers demonstrated normal velocities, but the patient was considered for cerebral angiogram given a high concern for vasospasm with his neurologic exam. Ticagrelor was stopped the following day.

\section{Results:}

The patient's tachypnea and mental status rapidly improved.

\section{Conclusions:}

Ticagrelor reversibly inhibits the platelet P2Y12 adenosine phosphate receptor and is indicated for prevention of cardiovascular events in adults with ACS. In patients with both cerebral hemorrhage and a need for antithrombotic therapy, this reversible agent may become more widely used. Dyspnea is a known side effect of ticagrelor, occurring in $13.8 \%$ of patients $(p<0.001) .1$ Dyspnea causes respiratory alkalosis and the resulting hypocapnea results in vasoconstriction. In this case, mental status change after administration of ticagrelor suggests that patients at risk for vasospasm may be particularly vulnerable to its side effects. 


\title{
Poster 243
}

\section{Nivolumab induced Lambert Eaton Myasthenic Syndrome in a patient with Metastatic Non-Small Cell Lung Cancer}

\author{
Stasia D. Rouse ${ }^{1}$, Rochelle R. Sweis ${ }^{1}$, Marisa M. Saint Martin ${ }^{2}$, Phillip P. DeChristopher ${ }^{3}$, Barak B. Bar ${ }^{1}$, \\ Jose J. Biller'.
}

\author{
${ }^{1}$ Department of Neurology, Loyola University Medical Center, Maywood, IL, USA, ${ }^{2}$ Blood Bank and \\ Apheresis Services, Department of Pathology, Maywood, IL, USA, ${ }^{3}$ Blood bank and Aphaeresis Services, \\ Loyola University Medical Center, Maywood, IL, USA.
}

\section{Introduction:}

Anti-programmed cell death 1 (PD-1) antibodies are an effective treatment option for NSCLC and other cancer entities. Anti PD-1 antibodies including nivolumab can induce immune-related adverse events (irAEs) in a number of organ systems. Neurological irAEs can be life-threatening and necessitate appropriate investigation and management by a neurologist. Myasthenic syndromes have rarely been described.

\section{Methods:}

Here we present a case of a man who developed a Lambert Eaton myasthenic syndrome thought to be a nivolumab-related immune adverse event.

\section{Results:}

A 90-year-old man on nivolumab for metastatic NSCLC developed asymmetrical ptosis followed by facial diplegia, dysarthria and dysphagia and fatigable limb weakness. He had clinical features of Lambert Eaton myasthenic syndrome with striking truncal, shoulder and pelvic girdle fatigable weakness that demonstrated a temporary increase in strength during the first few contractions. He developed neuromuscular respiratory failure requiring noninvasive positive pressure ventilation. His muscle reflexes were initially absent and after repeated attempts could be elicited. His MRI brain was normal, CSF was within normal limits and his serum and CSF antibodies against Ach Receptors, MUSK and Voltage gated Calcium Channels were negative. He weakened after an initial $1 \mathrm{mg} / \mathrm{kg}$ dose of prednisone. Plasma exchange resulted in a significant improvement of his weakness and the ability to wean the patient off noninvasive positive pressure ventilation.

\section{Conclusions:}

It is important to recognize that neurologic immune-related adverse events associated with nivolumab can cause Lambert Eaton myasthenic syndrome. Early recognition and aggressive treatment with plasma exchange can be life-saving. 


\section{Poster 244}

\section{Cerebral Microbleeds Manifesting as Stroke Symptoms During Extracorporeal Membrane Oxygenation}

Soo Jeong ${ }^{1}$, Hyo-Jae H. Kim ${ }^{1}$, Han-A H. Kim¹, Ji Man J. Hong ${ }^{2}$, Sang-Beom S. Jeon ${ }^{1}$.

${ }^{1}$ Department of Neurology, Asan Medical Center, University of Ulsan College of Medicine, Seoul, Korea, Republic of, ${ }^{2}$ 2Department of Neurology, School of Medicine, Ajou University, Suwon-si, Korea, Republic of.

****Permission was not granted to print this abstract**** 
Poster 245

\title{
Acute West Nile Virus Meningoencephalitis Diagnosed via Metagenomic Deep Sequencing of Cerebrospinal Fluid in a Renal Transplant Patient
}

\author{
Lara L. Zimmermann ${ }^{1}$, Michael M. Wilson ${ }^{2}$, Hannah H. Sample ${ }^{2}$, Emily E. Crawford ${ }^{3}$, Lillian L. Khan ${ }^{3}$, \\ Priya P. Soni ${ }^{4}$, Joseph J. DeRisi.
}

${ }^{1}$ University of California, Los Angeles, Los Angeles, CA, USA, ${ }^{2}$ Department of Neurology, University of California, San Francisco, San Francisco, CA, USA, ${ }^{3}$ Department of Biochemistry and Biophysics, University of California, San Francisco, San Francisco, CA, USA, ${ }^{4}$ University of California, Los Angeles, Los Angeles, USA.

\section{Introduction:}

Over the past 20 years, the Americas have experienced waves of emerging and re-emerging arboviruses that cause neuroinvasive disease, including West Nile virus, Chikungunya virus, Zika virus, and Dengue virus. These viruses pose great challenges for traditional candidate-based infectious disease diagnostics that already fail to identify a causative pathogen in approximately $50 \%$ of encephalitis cases.

\section{Methods:}

We present the case of a 14 year-old girl with a history of renal transplant managed with mycophenolic acid, tacrolimus and prednisone who presented to an emergency department with two days of high fevers, chills, upper back, neck pain and rash followed by encephalopathy. One month prior to presentation she attended summer camp by a lake in the Angeles National Forest, California. Her hospital course was complicated by status epilepticus. Cerebrospinal fluid (CSF) analysis demonstrated a mixed neutrophilic and lympocytic pleocytosis. MRI of the brain demonstrated symmetric T2 hyperintensities and edema in the bilateral thalami and leptomeningeal enhancement in the thalamus, cerebellum, brainstem, cervical spine and caudal equine. An extensive diagnostic work-up for infectious causes of encephalitis was performed and only identified Epstein-Barr virus.

\section{Results:}

A $500 \mu \mathrm{L}$ sample of CSF was submitted for unbiased metagenomic deep sequencing (MDS) under a research protocol. Unbiased MDS of RNA extracted from her CSF and processed through a custom bioinformatics pipeline identified West Nile virus. Subsequently, convalescent serum serologies confirmed West Nile virus infection.

\section{Conclusions:}

This case provides a first proof-of-principle that MDS can detect even low level arbovirus burden in the CSF of a patient with acute meningoencephalitis. Given the rapidly changing landscape of viral causes of encephalitis in the Americas, the ability of MDS to comprehensively detect a huge array of microbes with a single assay may make it an optimal method for early identification of emerging causes of viral encephalitis, including in the transplant patient population. 
Poster 246

\title{
Cerebral Phaeohyphomycosis caused by Cladophialophora bantiana -A Case Report and Review of Treatment Options.
}

\author{
Zaid Aljuboori ${ }^{1}$, Rob R. Hruska ${ }^{2}$, Haring H. Nauta ${ }^{1}$. \\ ${ }^{1}$ university of louisville/ Neurosurgery, louisville, KY, USA, ${ }^{2}$ university of louisville / Neurosurgery, \\ louisville, KY, USA.
}

\section{Introduction:}

Cladophialophora bantiana is a dematiaceous mold with a predilection for causing central nervous system infection, particularly in normal hosts. There is no standard therapy and mortality rates from this disease remain extremely high approaching $70 \%$.

\section{Methods:}

Here we describe a case involving a 59 year-old immunocompetent man who presented with new onset seizures. Brain imaging revealed bifrontal ring enhancing lesions concerning for abscess. The patient underwent surgical debridement of the lesions and bilateral intracavitary treatment with Amphotericin B using Ommaya reservoirs for several months.

\section{Results:}

After approximately 16 months of treatment which included surgical debridement, oral voriconazole and intracavitary Amphotericin B, our patient is off all antifungals and no longer receiving intracavitary treatment. He remains fully functional with a nonfocal neurologic exam, being monitored with serial brain MRIs.

\section{Conclusions:}

Due to rare incidence of cerebral phaeohyphomycosis, there are no clinical trials to help formulate standardized treatment guidelines despite its high mortality. This case places emphasis on an early aggressive multimodal approach for treatment of cerebral phaeohyphomycosis using a combination of surgical debridement, intracavitary antifungal injection, and oral antifungal therapy. 


\title{
Far reaching consequences of Carcinoid tumor of the middle ear (CTME) - the first reported case of CTME with dural sinus thrombosis and distant metastasis
}

\author{
Biplab K. Saha ${ }^{1}$, Himani H. Sharma ${ }^{2}$, Kristin K. Fless ${ }^{2}$, Paul P. Yodice ${ }^{2}$, Nirav N. Mistry², Fariborz F. \\ Rezai $^{2}$, Vagram V. Ovnanian ${ }^{2}$.
}

${ }^{1}$ Saint Barnabas Medical Center/ Department of Medicine, Livingston, NJ, USA, ${ }^{2}$ Saint Barnabas Medical
Center/Department of Medicine, Livingston, NJ, USA.

\section{Introduction:}

Neuroendocrine tumor (NET) of middle ear is extremely rare. Many names have been ascribed to these seemingly benign neuroendocrine lesions including middle ear adenoma (MEA), adenomatous tumor and carcinoid tumor (CT). 'Neuroendocrine adenoma' has also been used to better describe the histologic nature of these tumors. Here we present the first case of Carcinoid tumor of middle ear (CTME) complicated by dural sinus thrombosis and bony metastasis.

\section{Methods:}

A 73 year old man presented with right sided facial palsy for 4 hours and progressive hearing loss for 2 months. Physical examination revealed perforated right tympanic membrane with visible purulent material. CT scan of the head showed a small right cerebellar infarct. The MRI demonstrated right cerebellar hemorrhagic venous infarct and a heterogeneous middle ear mass extending into the internal auditory canal. MRV revealed thrombus in the right sigmoid and transverse sinus.Cervical spine MRI revealed diffuse osseous metastases. The histology and immunohistochemistry (IHC) after surgical resection was consistent with CTME. Proliferation rate of $>75 \%$ was seen by Ki67 staining. He suffered massive intracranial bleed on heparin therapy and passed away.

\section{Results:}

The NETs of head neck region are divided in 3 categories based on histology and IHC. 1) Well differentiated CT, 10/10 HPFs and Ki-67>20\%. Although regional metastasis is not uncommon, only 2 cases of distant metastasis have been reported in the past. Our patient was diagnosed to have atypical carcinoid but the Ki-67 was $>70 \%$ which is consistent with small cell cancer.

\section{Conclusions:}

The new classification system that takes the IHC and presence of metastasis into consideration to classify these tumors is much more clinically relevant. More research is necessary to find out the biological markers for better prognostication of this rare cancer. 


\section{Poster 248}

\section{Does Neurocritical Care Need to Improve Outreach to non-Neuro Specialties?}

Firas Abdulmajeed, MB. ChB, Bart B. Nathan, MD, FCNS.

University of Virginia/ Department of neurology, Charlottesville, VA, USA.

\section{Introduction:}

The number of Neuro- critical care(NCC) fellowship positions has been increasing yearly. The number of applicants has plateaued over the last 3 years, leaving many programs with unfilled fellowship positions. The demand for neurointensivists is on the rise. Trainees have come from Neurology traditionally, with a limited number from Internal Medicine (IM). We hypothesize that the relative paucity of IM fellows was in part due to insufficient knowledge of NCC.

\section{Methods:}

We surveyed IM residency programs in the United States, asking program directors to forward our survey to their residents. On another survey, NCC fellowship directors were asked: 1) how many trainees and how many faculty hires from IM and/ or EM have they had within the last five years

\section{Results:}

Internal Medicine residencies survey results: We obtained 102 individual responses, 98 responses were complete. How long is the NCC fellowship? N=100 12\% 1 year $41 \% 2$ years $47 \% 3$ years Residents of what specialty can apply to a NCC fellowship? $\mathrm{N}=97.73 \%$ (neurology, neurosurgery, anesthesiology, IM and EM) $11 \%$ neurology only $15 \%$ neurology, neurosurgery and anesthesia Knowledge of San Francisco matching system? N=92 48\% yes. 52\% No Do you know about the application cycle for the NCC match? $\mathrm{N}=93$ 10\% Yes. 90\% No Knowledge of Emergency Neurological Life Support? N=92 7\% Yes 93\% No NCC Fellowships' directors survey results: 20 of the 52 programs responded Fellows with IM/EM background that were trained within the last 5 years: $12 / 10$ Neuro-Intensivists with IM/EM background hired: $6 / 7$

\section{Conclusions:}

IM residents appear to have little knowledge of NCC fellowship. A lack of awareness of ENLS could affect the quality of care provided for neurological emergencies. Additionally, for the specialty to grow and fill unmatched fellowship positions, current training and outreach strategies to non-neurology trainees may need to be improved 
Poster 249

Transverse Myelitis due to Coxsackie B3/B4

Sung M. Cho.

Neurological Institute, Cleveland Clinic, Cleveland, OH, USA.

\section{Introduction:}

Acute transverse myelitis is an acquired inflammatory spinal cord disorder, which can be due to infection, autoimmune disorders, or malignancy, however, the cause is often unknown despite an extensive workup.

\section{Methods:}

We describe a rare case of acute transverse myelitis caused by Coxsackie B3/B4 virus.

\section{Results:}

A 36-year-old male with no past medical history presented with a viral prodrome of flu-like symptoms followed by severe headache, neck stiffness, photophobia, encephalopathy and paraplegia. MRI of the brain with contrast was unremarkable, but MRI of the spine showed an extensive longitudinal, nonenhancing T2 cord signal change from C6-T11 without hemorrhagic components. Lumbar puncture revealed 203 RBC, 214 WBC, 143 protein, and 46 glucose (95 mg/dL serum glucose). Extensive serum and CSF work-up was negative for HIV, WNV, EBV, CMV, Lyme, VZV, HSV, NMO antibody, ACE, paraneoplastic panel, cytology, Cryptococcus, and CSF bacterial and fungal cultures. CT chest and blind transbronchial needle lymph node biopsy were negative for sarcoidosis. Serum Coxsackie B3 (1:320) and B4 (> 1:640) antibody titers were significantly elevated. The patient was treated with intravenous methylprednisolone $1000 \mathrm{mg}$ for 5 days along with plasmapheresis for 5 sessions. The patient had improvement in lower extremity strength during his hospital stay and was discharged to a rehab facility on a steroid taper. At one-month follow up, the patient had complete recovery of lower extremity strength as well as bowel and bladder function and was ambulatory. At 3-month follow-up, MRI and CSF studies were markedly improved.

\section{Conclusions:}

Transverse myelitis due to Coxsackie has been reported in serotypes B2, B5, A9, A10, however only three cases of B3 or B4 related transverse myelitis have been reported and this is the first case, to our knowledge, with both B3 and B4 related transverse myelitis. 


\title{
Poster 250
}

\section{Inadvertent Intraventricular Contrast Injection: A Case Report and Systematic Review of Unintended External Ventricular Drain Injections}

\author{
Ayush Batra ${ }^{1}$, Kyle K. Wu ${ }^{2}$, David D. Lerner ${ }^{3}$, Starane S. Shepherd ${ }^{3}$, Rose R. Du², Galen G. Henderson ${ }^{4}$. \\ ${ }^{1}$ Brigham and Women's Hospital \& Massachusetts General Hospital / Neurology, Boston, MA, USA, ${ }^{2}$ \\ Brigham and Women's Hospital / Neurosurgery, Boston, MA, USA, ${ }^{3}$ Brigham and Women's Hospital \& \\ Massachusetts General Hospital/ Neurology, Boston, MA, USA, ${ }^{4}$ Brigham and Women's Hospital / \\ Neurology, Boston, MA, USA.
}

\section{Introduction:}

External ventricular drains (EVDs) are necessary for select patients admitted to the Neurointensive Care Unit (NICU). EVDs are critical to the management of diseases such as subarachnoid hemorrhage, traumatic brain injury, and acute hydrocephalus. We report a case of a woman with poor-grade aneurysmal subarachnoid hemorrhage who experienced inadvertent intraventricular non-iodinated contrast injection during vasospasm evaluation with CT angiography. We provide a review and analyses of adverse EVD related injections reported in the literature and summarize management recommendations.

\section{Methods:}

A PubMed search was performed for unintended EVD injections from 1927 to 2016 . Unique cases were selected and classified by the type of inadvertent injection and location of the event. Acute management was categorized by the use of EVD manipulation, lumbar drain placement, and supportive medical therapies. Cases involving ventriculoperitoneal shunts, Ommaya reservoirs, or other intrathecal device systems were excluded.

\section{Results:}

A total of seven unique cases were identified, four involving contrast administration and three involving medication administration. The sentinel event in five cases occurred outside of the NICU. Acute management with EVD manipulation was employed in six cases; three of these cases also used lumbar drains. Three cases necessitated intubation. Our patient received prophylactic dexamethasone and levetiracetam, underwent immediate EVD manipulation, and placement of a lumbar drain. Continuous EEG monitoring and daily head CTs were performed until intraventricular clearance was noted at 72 hours.

\section{Conclusions:}

Acute management of inadvertent intraventricular injections entails immediate EVD manipulation and possible lumbar drain placement to facilitate rapid CSF clearance of injected substances. Intubation may be required immediately depending on exposure. Inadequate distinction between compatible drain tubing and relative inexperience of providers managing the EVDs likely contributed to the errors in these cases. The increased incidence of these events outside of the NICU suggests additional safety measures may be warranted when patients with EVDs travel off-unit. 
Poster 251

\title{
An unusual presentation of isolated brain abscess in non-traumatic convexal subarachnoid hemorrhage: A case report
}

\author{
Amandeep S. Dolla', Alan A. Wang ${ }^{2}$, Muhammad M. Athar ${ }^{3}$. \\ ${ }^{1}$ Thomas Jefferson Hospital/ Dept. of Neurology, Philadelphia, PA, USA, ${ }^{2}$ Thomas Jefferson \\ Hospital/Dept of Neurology, Philadelphia, PA, USA, ${ }^{3}$ Thomas Jefferson Hospital/Dept of Neurological \\ surgery, Philadelphia, PA, USA.
}

\section{Introduction:}

To present a unique case of isolated brain abscess presenting as non-traumatic convexal subarachnoid hemorrhage (CSAH) six days before radiologic signs could be seen. To our knowledge only one other case of cSAH due to brain abscess has been reported thus far.

\section{Methods:}

Description of a clinical case

\section{Results:}

A 42-year old man with no past medical history or prior trauma presented with acute onset of transient left hemianesthesia lasting ten minutes. Computed tomography (CT) of the head revealed cSAH. We were presented with a diagnostic dilemma when magnetic resonance imaging (MRI) of the brain, magnetic resonance angiography (MRA) of the head and neck, magnetic resonance venography (MRV) and conventional angiography failed to show the cause of cSAH. The patient was discharged in a stable condition, but returned six days later with worsening symptoms, including left hemiparesis. Repeat MRI with contrast revealed a cerebral abscess in the same location as the prior cSAH.

\section{Conclusions:}

cSAH without clear evidence of trauma due to abscess is a rare occurrence. We suggest in cases of cSAH where imaging techniques present no abnormalities, follow-up imaging within seven days should be considered. 


\title{
Poster 252
}

\section{Delayed Plantar Extension with Noxious Nail Bed Stimulation in Brain Death}

\author{
Jonathan Marehbian, Diane D. Chan, David D. Greer.
}

Yale New Haven Hospital, Department of Neurology, New Haven, CT, USA.

\section{Introduction:}

Spinally-mediated reflex movements can be present in brain dead patients. However, abnormal movements have long been a challenge in the clinical determination of brain death. In this report, we describe delayed plantar extension with noxious nail bed stimulation that has not been previously described in brain death.

\section{Methods:}

A 28-year-old male suffered severe anoxic brain injury following respiratory failure due to heroin overdose. His clinical exam and apnea testing were consistent with brain death with the exception of a reproducible delayed plantar extension with noxious nail bed stimulation.

\section{Results:}

Ancillary testing with technetium 99m nuclear scan (SPECT) demonstrated no cerebral blood flow, confirming that the finding was spinally-mediated.

\section{Conclusions:}

Novel movements are important to document in order to aid in the timely determination of brain death, and to avoid unnecessary and potentially confounding ancillary testing. The mechanism underlying delayed plantar extension is likely spinally mediated. 
Poster 253

\title{
Somatosensory evoked potentials as Neurmonitoring Tool in NeurolCU
}

\author{
Anantha Ramana Vellipuram ${ }^{1}$, Christopher C. Newey ${ }^{2}$, Agnieszka A. Ardelt ${ }^{1}$, Vernon V. Towle ${ }^{3}$. \\ ${ }^{1}$ Dept of Neurology. University of Chicago, Chicago, IL, USA, ${ }^{2}$ Dept of Neurology. University of Missouri , \\ Columbia, MO, USA, ${ }^{3}$ Dept pf Neurology. University of Chicago, Chicago, IL, USA.
}

\section{Introduction:}

Somatosensory evoked potentials (SSEPs) are a sensitive, minimally invasive technique used to identify injury from the posterior columns of the spinal cord to the somatosensory cortex. The role of SSEPs as a neuromonitoring tool, in the neurolCU has not been well established. We present a case using SSEPs as a neuromonitoring tool illustrating electrical improvement along with clinical and radiographical improvement in a symptomatic Chiari I malformation.

\section{Methods:}

Case Report

\section{Results:}

18 year old female who was 2 months postpartum after vaginal delivery with epidural analgesia presented with headaches, diplopia and nausea/vomiting. After arrival to ER, she acutely developed flaccid quadriparesis with ophthalmoplegia and loss of airway while awake and following commands. Given the concern for intracranial hypotension, we administered mannitol, hyperventilated and placed in Trendelenburg position. Head CT showed cisternal effacement in the setting of a likely pre-existing Chiari 1 malformation with cerebellar tonsillar. Decompressive surgery was not an option initially given her dysautonomia and neurologic instability whenever the patient was not in Trendelenburg. During her prolonged course, she had two MRIs of her brain and spinal cord which showed Chiari I malformation with syrinx at C4 and presyrinx down to T2. There was cervical spine venous engorgement and CSF block at the level of the foramen-magnum. She was evaluated with serial SSEPs which initially showed low amplitude N20 response that improved with her clinical improvement.

\section{Conclusions:}

SSEP is a minimally invasive method to electrically assess the somatosensory pathway integrity from the spinal cord, brainstem and cortex. Given its sensitivity to the function of the dorsal columns of the spinal cord and medial lemniscus of the brainstem, SSEPs may be a useful monitoring adjunct to follow the evolution of posterior fossa lesions in patients that may not tolerate other means of monitoring and/or transportation, such as MRI. 


\title{
Poster 254
}

\section{latrogenic Vessel Perforation in Neuroendovascular Interventions and its Management in the Neurointensive Care Unit: Targets of therapy.}

\author{
Michelle S. Nunes ${ }^{1}$, Pedro P. Aguilar-Salinas ${ }^{2}$, Douglas D. Gonsales ${ }^{3}$, Eric E. Sauvageau ${ }^{2}$, Ricardo R. \\ Hanel. \\ ${ }^{1}$ Baptist Health, Neurointensive Care Unit, Jacksonville, FL, USA, ${ }^{2}$ Baptist Health, Lyerly Neurosurgery, \\ Jacksonville, FL, USA, ${ }^{3}$ Baptist Health, Lyerly Neurosurgery, Jacksvonville, FL, USA.
}

\section{Introduction:}

Vascular perforation during a neuroendovascular procedure is an unexpected and feared complication, which can lead to fatal outcomes. A prompt recognition and initiation of treatment are paramount. Endovascular strategies to address this complication have been widely described. However, the goals of therapy in the neurointensive care unit (NeurolCU) remain unclear. We report two cases in which endovascular strategies associated with aggressive intensive care resulted in a good clinical outcome at discharge.

\section{Methods:}

Case Report.

\section{Results:}

Vessel perforation occurred in 2 patients during neuroendovascular interventions: a left-carotid stenting and an ICA-aneurysm embolization with balloon-assisted coiling. Once contrast extravasation was demonstrated, heparin was immediately reversed and endovascular strategies were performed to minimize the complications. SAH was present in the initial head CT in both cases. In the NeurolCU, targets of therapy were A) systolic blood pressure (SBP) $<110 \mathrm{mmHg}, \mathrm{B}$ ) mechanical ventilatory support, and $C$ ) seizures and vasospasm prophylaxis with phenytoin and nimodipine respectively. One of the patients developed mild hydrocephalus and left upper extremity weakness, with MRI showing tiny right hemispheric strokes, while the other one, did not show any focal deficit. Both patients were discharged home few days later with mRS of 2 and 0 , respectively.

\section{Conclusions:}

latrogenic vascular perforation is an uncommon complication that occurs secondary to inadequate manipulation of the catheter, guide wire, devices, or forceful contrast injection. Clinical care strategies aim to prevent fatal outcomes. We recommend reversing heparin; provide an adequate ventilatory support, maintain a strict control over the SBP, and seizure and vasospasm prophylaxis. But, larger studies are required to determine the standard of care since endovascular therapy is rapidly becoming first-line of treatment for neurovascular conditions. 
Poster 255

\title{
A CASE OF CEREBRAL HYPERPERFUSION SYNDROME AFTER SPONTANEOUS RECANALIZATION OF CAROTID INTRA-STENT THROMBOSIS.
}

\author{
Michelle S. Nunes ${ }^{1}$, Pedro P. Aguilar-Salinas ${ }^{2}$, Ricardo R. Hanel ${ }^{2}$, Mohamad M. Chmayssani ${ }^{3}$. \\ ${ }^{1}$ Baptist Health, Neurocritical Care Unit, Jacksonville, FL, USA, ${ }^{2}$ Lyerly Neurosurgery, Baptist Health, \\ Jacksonville, FL, USA, ${ }^{3}$ Baptist Health, Neurocritical Care Unit, Jacksonville, FL, USA.
}

\section{Introduction:}

Cerebral Hyperperfusion Syndrome (CHS) symptoms range from severe unilateral headache to seizures, focal symptoms and intracerebral hemorrhage, usually occurring follow carotid endarterectomy (CEA)/ carotid stenting (CAS). We describe a case of a patient who developed CHS after spontaneous recanalization of carotid intra-stent thrombosis.

\section{Methods:}

Case report.

\section{Results:}

68 years old African American male who had recently placed left cervical stent was transferred to Baptist Medical Center with new evidence of intra-stent thrombosis. He was started on heparin drip and SBP was augmented with levophed for SBP $>160$. Two days later, the patient developed excruciating headache followed 6 hours later by acute onset of right upper extremity and face twitching associated with severe hypertension. The BP was controlled, protamine was given to reverse heparin and Keppra was loaded to treating seizures. CTA at this time showed recanalization of carotid in-stent stenosis and CTP confirmed hyperperfusion of frontal and parietal lobe with neither evidence of new ischemic area nor bleeding. Fortunately, inspite a delay in diagnosis by 6 hours, patient did not suffer intracranial hemorrhage.

\section{Conclusions:}

Most patients who develop CHS will have complete recovery if it is discovered and treated early. Aggressive prophylactic blood pressure control is the main treatment. For those who are diagnosed late and those progressing to $\mathrm{ICH}$, the prognosis can be devastating with mortality rates up to $50 \%$. Considering the importance of blood pressure control in the cerebral hemodynamic, studies have been done, trying to find a better tool to predict the best BP target in order to prevent CHS. Near Infrared Spectroscopy (NIRS) is a non-invasive and reliable technique that monitor the cerebral hemodynamic. Had NIRS been deployed during anticoagulation, the diagnosis of cerebral hyperperfusion syndrome would have been made at the onset of headaches and would have avoided the development of seizures and potentially a life threatening hemorrhage. 


\title{
Poster 256
}

\section{The Triphasic Response: Water imbalance after neurosurgery : A case report}

\author{
Bibhukalyani Das, Indranil I. Ghosh.
}

Institute of Neurosciences Kolkata, Kolkata, India.

\section{Introduction:}

Water balance disorders after neurosurgery are well recognized, but detailed reports of the triphasic response are scarce. We describe a 30-year-old woman, who developed the triphasic response with hyper and hyponatraemia after resection of craniopharyngeoma.

\section{Methods:}

A 30-year-old female (no previous medical history, no medication, normal electrolytes and endocrine parameters) with MRI showing s/o craniopharyngeoma underwent neurosurgery using a subfrontal approach to resect the craniopharyngeoma while leaving the pituitary stalk intact (according to the surgical notes). Within a few hours of an uneventful postoperative recovery she started having polyuria and hypoosmolar urine with hypernatremia. Being normoglycaemic and not on any diuretics a diagnosis of central diabetes insipidus was made treated with Desmopressin nasal spray and drinking water ad libitum. Improvement occurred over the next postoperative day when desmopressin was discontinued. On the third day she developed with hypoosmolar hyponatremia along with seizure treated with $3 \%$ saline and fuid restriction. Two days later polyuria returned and was ultimately discharged with desmopressin tablets and advice to take fluids ad libitum.

\section{Results:}

NA

\section{Conclusions:}

This case illustrates the dramatic and sudden changes in water balance that may occur after neurosurgery. The pathophysiology of the triphasic response appears to be early hypothalamic dysfunction, subsequent release of vasopressin from the degenerating pituitary and, finally, depletion of vasopressin stores. It has been difficult to identify patients at risk, but predisposing factors appear to relate both to the disease (macroadenoma, microadenoma, craniopharyngioma) and to the surgery (degree of manipulation). Successful prevention probably involves a psychological switch by not waiting until frank dysnatraemia has developed, but to act as soon as urine output and tonicity change. This requires an index of suspicion for treating and consulting physicians and specific instructions to nursing staff, especially in nonintensive care settings. 
Poster 257

\title{
Ketamine for Refractory Status Epilepticus: Report of Adverse Cardiac Events
}

\author{
Lauren Koffman ${ }^{1}$, Salia S. Farrokh ${ }^{2}$, Ho Yan H. Yiü, John J. Lewin III', Romergryko R. Geocadin ${ }^{3}$, \\ Wendy W. Ziai'.
}

\footnotetext{
${ }^{1}$ The Johns Hopkins Hospital/ Anesthesiology and Critical Care Medicine, Baltimore, MD, USA, ${ }^{2}$ The Johns Hopkins Hospital/ Critical Care and Surgery Pharmacy, Baltimore, MD, USA, ${ }^{3}$ The Johns Hopkins Hospital/ Critical Care and Surgery Pharmacy, Baltimore, MD, USA.
}

\section{Introduction:}

Refractory status epilepticus (RSE) has high mortality and is difficult to treat. When traditional therapies fail ketamine may be considered. Ketamine is associated with limited reports of adverse cardiac events during anesthesia, but not during treatment for RSE. We evaluated occurrences of cardiac arrhythmias associated with ketamine.

\section{Methods:}

Retrospective chart review of neurocritical care patients in a tertiary academic medical center who received ketamine infusion for RSE between October 2010 and April 2016.

\section{Results:}

Ten patients were admitted to a neurologic intensive care unit and received ketamine infusion for RSE. Etiology of RSE included autoimmune/infectious process (8), ischemic stroke (1) and subarachnoid hemorrhage (1). Of the ten patients who received ketamine, three had documented cardiac events without prior cardiac history. One patient remained clinically stable and did not require intervention. Another patient required escalating doses of ketamine infusion (maximum $6 \mathrm{mg} / \mathrm{kg} / \mathrm{hr}$ ) for RSE secondary to presumed leptomeningeal disease, had an asystolic event and expired. The third patient was on low dose ketamine $(0.07 \mathrm{mg} / \mathrm{kg} / \mathrm{hr})$ for RSE secondary to subarachnoid hemorrhage, and developed multiple arrhythmias including recurrent episodes of asystole. Once ketamine was discontinued the patient stabilized.

\section{Conclusions:}

Arrhythmias are not uncommon in critically ill patients, but this is the first report of cardiac arrest associated with the use of ketamine for RSE. Although sympathomimetic properties of ketamine may provide vasopressor sparing effects, which reduce the need for vasopressors to counteract the hypotension commonly seen with other anesthetics used in RSE, it may put patients at risk for cardiac arrhythmias. In addition, ketamine has direct negative ionotropic effects and may raise pulmonary artery pressures. Caution should be employed when ketamine is used in RSE in patients with other independent risk factors for cardiac events. 


\section{Poster 258}

\section{CNS Post Transplant Lymphoproliferative Disorder presenting as de novo Status Epilepticus: Case Report}

Avni M. Kapadia, Navin N. Kesari, Sanjeev S. Sivakumar, Chethan C. Venkatasubba Rao.

Baylor College of Medicine/Department of Neurology/Neurocritical Care, Houston, TX, USA.

\section{Introduction:}

Primary central nervous system post-transplant lymphoproliferative disorder (PCNS-PTLD) is a rare complication of solid organ transplant. PCNS-PTLD can present with multiple intracranial lesions and lobar involvement, with seizures reported in about $25 \%$ of patients. Status epilepticus (SE) in PCNSPTLD, is very sparsely reported.

\section{Methods:}

Review of prospectively maintained patient database identified one case of status epilepticus in a patient with CNS-PTLD. We present a case report with literature review.

\section{Results:}

A 74-year old Hispanic woman with a history of renal transplant 19 years prior, presented with episodic confusion and gait ataxia progressing over two weeks. She was on immunomodulation with Mycophenolate. Patient had witnessed periods of behavioral arrest. Continuous electroencephalography (cEEG) demonstrated right temporal sharps and 8-20 second epochs of bi-frontal 14-18 Hz activity, some of which were associated with non-stereotyped movements of her left shoulder and trunk, suggestive of SE. She received benzodiazepines followed by Levetiracetam (renal dose) and Phenytoin load for seizure control. MRI brain without contrast demonstrated multifocal infiltrative T2-hyperintense white matter lesions, most prominent in right temporal lobe. CSF analysis demonstrated 2 RBC, 63 (L 88\%) WBC, 80 protein, 71 glucose, culture and gram stain were negative. There were 4 unmatched CSF bands with an unremarkable cytology. CSF PCR was positive for EBV and viral load was detected at 1458 copies/nL. Other CSF microbial assays including JCV were negative. Stereotactic right temporal brain biopsy demonstrated areas of necrosis, axonal disruption, loss of myelin with polytypic plasma cells, CD20 and CD30 positive $B$ cells and CD3 positive $T$ cells on immunohistochemistry consistent with a diagnosis of polymorphic PTLD. Despite treatment with dexamethasone and rituximab, patient continued to remain critically ill and eventually received palliative measures.

\section{Conclusions:}

Among transplant recipients, PCNS-PTLD is rare but debilitating with varied neurological presentation. High degree of suspicion, early diagnosis and treatment are paramount for survival. 
Poster 259

\section{Epilepsy Surgery for New-Onset Super-Refractory Status Epilepticus: A Case Report}

Simona Ferioli, $M D^{1}$, Jordan J. Bonomo, $M D^{2}$, Molly M. O'Brien, $A C N P^{1}$, Christopher C. Zammit, $M D^{1}$, Daniel D. Kanter, $M D^{1}$, Michael M. Privitera, MD', Heather H. McKee, MD ${ }^{3}$, George G. Mandybur, MD', Brandon B. Foreman, $M D^{1}$.

${ }^{1}$ University of Cincinnati, Department of Neurology \& Rehabilitation Medicine, Cincinnati, OH, USA, ${ }^{2}$ University of Cincinnati, Department of Emergency Medicine, Cincinnati, OH, USA, ${ }^{3}$ University of Cincinnati, Comprehensive Epilepsy Center, Cincinnati, OH, USA, ${ }^{4}$ University of Cincinnati, Department of Neurosurgery, Cincinnati, OH, USA.

\section{Introduction:}

We report a rare case of new-onset super-refractory status epilepticus requiring advanced epilepsy surgery with good outcome.

\section{Methods:}

Case Report.

\section{Results:}

A 36 year-old veterinary technician with headache and fever for 2 days presented with altered mental status and myoclonic jerking. Initial LP showed 21 white cells and elevated protein $(71 \mathrm{mg} / \mathrm{dL})$. Recurrent clinical seizures occurred for 6 days prior to transfer to our institution. His exam demonstrated diffuse hyperreflexia and coma; EEG demonstrated up to $2.5 \mathrm{~Hz}$ frontally-predominant rhythmic delta but no unequivocal seizures. Extensive workup revealed no evidence of infectious, toxic, or immune-mediated encephalitis. MRI demonstrated bithalamic injury and a region of questionable periventricular nodular heterotopia in the right parietal region. Subsequently, he developed recurrent clinical and unequivocal electrographic seizures from the right parieto-occipital region. Five periods each lasting $>48$ hours of anesthesia-induced EEG burst suppression failed to stop seizures, qualifying him as super-refractory status epilepticus. After 6 weeks of failure to wean from anesthetia, invasive monitoring for seizure localization was carried out using strips and depth electrodes. Multiple seizures were recorded, localizing to the medial occipital lobe, which was subsequently resected along with the region of pathologyconfirmed heterotopia. Seizures gradually improved, requiring 7 anti-seizure drugs and a ketogenic diet. He regained consciousness with preserved higher cognitive functions (language, memory) and personality 6 months later as his antiseizure drugs were successfully decreased. His motor recovery was limited by critical illness myoneuropathy.

\section{Conclusions:}

New-onset focal super-refractory status epilepticus may respond to surgical resection in extreme cases. Close collaboration with a multidisciplinary team of epileptologists and neurosurgeons can lead to resolution of seizures and eventually recovery. 
Poster 260

\title{
Cortical Activation, Myoclonic Status and Multiple Subdural Hematomas
}

\author{
Flavio A. Carvalho' ${ }^{1}$ Paulo P. Monzillo², Gisele G. Silva². \\ ${ }^{1}$ Hospital Israeliat Albert Einstein, São Paulo, Brazil, ${ }^{2}$ Hospital Israelita Albert Einstein, São Paulo, Brazil.
}

\section{Introduction:}

Myoclonus caused by activation of cortical areas subjacent to multiple subdural hematomas is an unusual mechanism of epilepsy. We report the case of a patient with an extra axial bleeding and myoclonic seizures evaluated with ictal FDG-PET.

\section{Methods:}

Case Report

\section{Results:}

A 59 year old male was admitted to our hospital because of worsening symptoms of cardiac failure of Chagasic etiology. Due to hemodynamic instability he was treated with the placement of an intraortic balloon pump. Sixteen days after hospital admission, he presented intermittent generalized myoclonic jerks. On initial examination he was alert and oriented to time and place, had preserved strength in all limbs, although presenting with very frequent clusters of myoclonus. Initial investigation with a head CT showed multiple foci of extra axial bleeding, distributed over the frontal and parietal areas. The electroencephalogram (EEG) confirmed the suspected diagnosis of myoclonic seizures, exhibiting generalized polispike-slow wave complex. Due to the multiplicity of bleeding sites, with no obvious reason for spontaneous bleeding other than regular anticoagulation, the patient was submitted to a whole-body FDG-PET in order to exclude the possibility of dural metastatic implants. FDG-PET showed areas of cortical hypermetabolism adjacent to the bleeding foci, probably reflecting an epileptogenic mechanism of cortical activation. There was no evidence of hypermetabolism directly over the extra-axial areas of bleeding, what ruled out the hypothesis of dural metastasis. A diagnosis of spontaneous subdural hematomas associated to anticoagulation was given after all other causes were excluded. The patient was treated with sodium valproate and had sustained improvement of the myoclonic seizures. The bleeding areas were eventually reabsorbed, but the patient died from complications of cardiac failure.

\section{Conclusions:}

Our report is the first to illustrate the mechanism of cortical activation leading to epileptic status in a patient with multiple subdural hematomas detected by ictal FDG-PET. 
Poster 261

\section{Transient neurologic events in a patient with leptomeningeal metastases: Case report and literature review}

Christoph Stretz, Yan Y. Zhang.

Department of Neurology, Hartford Hospital, Hartford, CT, USA.

\section{Introduction:}

In patients with leptomeningeal metastases (LM) and elevated intracranial pressure (ICP), transient neurologic events secondary to plateau waves - temporary elevations in ICP - may occur. There is a paucity of clinical reports correlating video-EEG with definite or presumed plateau waves in patients with LM.

\section{Methods:}

Case report and literature review. Pubmed was queried for 'leptomeningeal metastases and EEG', 'leptomeningeal metastases and plateau waves' and 'leptomeningeal metastases and intracranial pressure'.

\section{Results:}

A 48-year-old woman was transferred to our hospital for further care of a pituitary microadenoma noted on outside imaging. She had a history of Hodgkin lymphoma treated with radio-chemotherapy. During her admission, she developed episodes of confusion and video-EEG monitoring was initiated. While there were no electrographic seizures, several events, between 5 to 10 minutes in duration, of delayed or absent verbal responses, eye rolling, staring and alternating gaze preferences to both sides were noted. All these coincided with abrupt onset of marked background slowing, evident through generalized $2-3 \mathrm{~Hz}$ delta, mixed with some theta frequencies. Review of outside and repeat imaging with MRI of the brain with contrast revealed brain and leptomeningeal metastases. Cerebrospinal fluid examination showed raised opening pressures and malignant cells, leading to a subsequent diagnosis of primary signet ring cell cancer. We found one additional report describing video-EEG correlates of presumed plateau waves in patients with LM.

\section{Conclusions:}

Our clinical description of transient neurologic events in this patient adds to the current literature of paroxysmal manifestations owing to raised ICP in patients with LM. Awareness of this clinical phenomenon may serve as a surrogate of raised ICP before clinical signs of the same develop in patients with LM. It may also help delineate the cause of raised ICP due to CNS metastases in a patient with a corresponding history of cancer. 


\section{Poster 262}

Pentobarbital-induced Myocardial Stunning in Status Epilepticus Requiring Extracorporeal Membrane Oxygenation

Tapan Kavi ${ }^{1}$, Donna D. Molaie ${ }^{2}$, Axel A. Rosengart ${ }^{1}$, Shouri S. Lahiri' ${ }^{1}$

${ }^{1}$ Neurology/Neurocritical Care, Los Angeles, CA, USA, ${ }^{2}$ Neurology, Los Angeles, CA, USA.

****Permission was not granted to print this abstract**** 
Poster 263

\title{
Terson's Syndrome: A Case Report and Literature Review
}

\author{
Jesse M. Robertson ${ }^{1}$, Vikas V. Grover'. \\ ${ }^{1}$ Legacy Health Internal Medicine Residency Training Program, Portland, OR, USA, ${ }^{2}$ Legacy Health, \\ Portland, OR, USA.
}

\section{Introduction:}

Background: Terson's Syndrome is the development of intraocular hemorrhage $(\mathrm{IOH})$ in association with subarachnoid hemorrhage (SAH). We report a case of Terson's syndrome and review the literature.

\section{Methods:}

Case Report: A 48yr woman presented with severe neck pain and somnolence. CT imaging showed Fisher Grade $3 \mathrm{SAH}$ with aneurysms in the right internal carotid and posterior communicating arteries. She developed low pressure hydrocephalus treated with ventriculostomy. On hospital day 12 she developed vision loss. Ophthalmologic examination demonstrated bilateral vitreous hemorrhages with near complete fundoscopic resolution by day 19 . Over the next 4 months she underwent pars plana vitrectomy (PPV) of the left eye two times. Current vision OD 20/20, OS 20/50.

\section{Results:}

Discussion: The incidence of Terson's syndrome among patients with SAH is documented to be between $8-40 \%$. In prospective studies, $\mathrm{IOH}$ was found in up to $28 \%$ of patients with $\mathrm{SAH}$ compared to only $3 \%$ in retrospective studies suggesting under-reporting. This is likely because $\mathrm{IOH}$ is found more often in higher severity bleeds where patients cannot self-report visual loss due to decreased LOC. Patients with IOH are more likely to have worse neurologic outcomes and die more often than those with lower grade bleeds without the development of $\mathrm{IOH}$. Along with fundoscopy, hand held ultrasound may be used for bedside diagnosis. ERM development is the most common intraocular complication from Terson's syndrome and occurs in $15-78 \%$ of patients. Complete or near complete return of visual acuity is less likely without surgical intervention. Visual acuity has been show to recover better and faster if PPV is performed within 90 days. There is no literature on incidence of visual loss after acute phase of SAH.

\section{Conclusions:}

Conclusions: Routine evaluation with imaging and fundoscopy may help in detecting $\mathrm{IOH}$ sooner in the clinical course and has the potential to decrease long-term morbidity. 


\title{
Poster 264
}

\section{Unintended Underfeeding in Neurocritical Care Patients from Tube Feeding Interruptions: Assessing the Metabolic Impact}

\author{
Angela Vizzini ${ }^{1}$, William W. Freeman ${ }^{2}$, Juan J. Canabal ${ }^{3}$, Pablo P. Moreno Franco ${ }^{3}$, Joshua J. Propst ${ }^{3}$. \\ ${ }^{1}$ Mayo Clinic Florida, Jacksonville, FL, USA, ${ }^{2}$ Mayo Clinic Florida/ Neurology, Neurosurgery and Critical \\ Care, Jacksonville, FL, USA, ${ }^{3}$ Mayo Clinic Florida/ Transplant Critical Care, Jacksonville, FL, USA.
}

\section{Introduction:}

latrogenic underfeeding in critically ill patients is often unrecognized and underestimated. An international prospective study of 201 critical care units showed patients received only $61.2 \%$ and $57.6 \%$ of prescribed calorie and protein needs. The inability to initiate enteral nutrition within 24-48 hours of ICU admission or frequent interruptions of the enteral regimen lead to insufficient nutrient delivery and a compounding energy deficit. An increase in infectious complications is associated with negative energy balance in patients with Subarachnoid Hemorrhage (SAH).

\section{Methods:}

A quality initiative project was developed at Mayo Clinic Florida to measure time to reach enteral nutrition target and common interruptions of enteral nutrition. The target subjects were mechanically ventilated patients in medical and transplant ICU; however the quality measure has recently extended to the Neurocritical Care unit. Data collected included clinical diagnosis, SOFA and APACHE II score, Subjective Global Assessment (SGA) score, Nutric score, enteral tube type and regimen, and reason and duration for interruption of nutrition.

\section{Results:}

A 76 year old subject with SAH and Posterior fossa AVM resection was deemed low nutrition risk with SGA score A and Nutric score 4. Enteral nutrition was initiated via nasoenteric tube within 25 hours of intubation. Target enteral goal rate was reached within 16 hours. The patient received $100 \%$ of calorie/protein needs 9 of the 12 days. The most common enteral interruption was for procedure; primarily head CT, for longest duration of 155 minutes.

\section{Conclusions:}

The interruption of enteral nutrition in Neurocritical care patients is likely unavoidable due to procedures. These disruptions, however, need not result in iatrogenic underfeeding. Neurocritical care units may utilize volume-based enteral protocols to allow nurses to compensate for lost nutrition with increased enteral rate. 


\title{
Poster 265
}

\section{Veno-venous ECMO as Rescue Therapy for Severe Neurogenic Pulmonary Edema due to Aneurysmal Subarachnoid Hemorrhage}

\author{
Marin Darsie ${ }^{1}$, Rhonda R. Cadena ${ }^{1}$, Deanna D. Sasaki-Adams ${ }^{2}$, Anthony A. Charles ${ }^{3}$, Casey C. Olm- \\ Shipman ${ }^{1}$. \\ ${ }^{1}$ University of North Carolina-Chapel Hill/Department of Neurology, Chapel Hill, NC, USA, ${ }^{2}$ University of \\ North Carolina-Chapel Hill/Department of Neurosurgery, Chapel Hill, NC, USA, ${ }^{3}$ University of North \\ Carolina-Chapel Hill/Department of Surgery, Chapel Hill, NC, USA.
}

\section{Introduction:}

Neurogenic pulmonary edema is challenging to manage in the context of aneurysmal subarachnoid hemorrhage (aSAH) due competing priorities between organ systems. We present a case of refractory neurogenic pulmonary edema due to aSAH necessitating extracorporeal membrane oxygenation (ECMO).

\section{Methods:}

Case report.

\section{Results:}

A 30 year-old female with a history of hypertension and diabetes presented neurologically intact with $\mathrm{HH} 2 \mathrm{~F} 3 \mathrm{aSAH}$ due to a left posterior communicating artery aneurysm. She underwent coil embolization on $\mathrm{SAH}$ day 2 and remained intubated after the procedure due to development of flash pulmonary edema. Transthoracic echo demonstrated normal left ventricular function. On SAH day 3 after unplanned extubation, she was temporized on noninvasive ventilation until reintubation the following day. She progressed to severe ARDS requiring high-dose sedatives and paralytics which obscured her neurologic exam. On SAH day 7, a 9 day course of intrathecal nicardipine was initiated for elevated left MCA transcranial doppler velocities. On SAH day 8 , her respiratory status further declined and veno-venous ECMO was initiated as rescue therapy after head CT did not demonstrate new hemorrhage or infarct. Throughout the 7 day ECMO course, a low-dose heparin infusion was utilized along with vasopressors to optimize cerebral perfusion pressure (CPP). She was decannulated on SAH day 15. Surveillance CT head demonstrated left-sided ischemic infarcts in multiple vascular territories. On SAH day 42, she was discharged to an outside facility for ventilator weaning. Upon discharge, she was alert and followed commands with her left arm, however she was aphasic without movement of her other extremities.

\section{Conclusions:}

Veno-venous ECMO was performed in an aSAH patient after coil embolization. Although the patient did not develop intracranial hemorrhage, her course was complicated by severe vasospasm and delayed cerebral ischemia (DCl). While ECMO is a rescue therapy for severe hypoxemia, its effect on CPP remains uncertain and may potentiate $\mathrm{DCl}$. 


\title{
Poster 266
}

\section{Rethinking Myoclonic Status Epilepticus in the Young: A Case Report}

\author{
Adam Lewis ${ }^{1}$, Matthew M. Holtkamp ${ }^{2}$, Philip P. Eye ${ }^{2}$. \\ ${ }^{1}$ Walter Reed National Military Medical Center, Bethesda, MD, USA, ${ }^{2}$ Walter Reed NMMC, Bethesda, \\ $M D, U S A$.
}

\section{Introduction:}

To present a case of early onset myoclonic status epilepticus (MSE) after cardiopulmonary arrest with incomplete resolution of myoclonus and good cognitive outcome.

\section{Methods:}

A 20 year-old man presented status post cardiopulmonary arrest and CPR in the field with return of spontaneous circulation (ROSC) after arrival to the hospital and cardioversion. The patient was intubated and treated with therapeutic hypothermia, but developed clinical MSE with normal EEG within 12 hours. $\mathrm{He}$ was aggressively managed with propofol, levetiracetam, and fentanyl. Initial MRI demonstrated diffuse hypoxic ischemic injury. MRI on the 9th day of admission demonstrated improvement but a new white matter lesion in the splenium of the corpus callosum.

\section{Results:}

After multiple unsuccessful attempts to discontinue fentanyl and 15 days of treatment, the patient was given a poor prognosis based on the 2006 AAN MSE Practice Parameters and was placed on do not escalate care orders. Propofol was slowly decreased; however the patient improved significantly throughout hospitalization with improved language and cognitive examination and only mild residual reflex myoclonus at the time of discharge. MRI imaging had completely resolved by the 19th day of hospitalization. The patient's final diagnosis is Lance-Adams syndrome of action myoclonus incompletely controlled with levetiracetam.

\section{Conclusions:}

Aggressive and prolonged treatment including therapeutic hypothermia in young patients with early onset MSE was effective despite AAN practice parameters. This patient survived with good cognitive outcome and with relatively modest deficits. Further research is needed to assess whether improvements in intensive care unit capabilities over the past decade may contribute to improved outcome in young patients with cardiac arrest and whether practice parameters should be revised. 


\section{Financial Disclosures}

\begin{tabular}{|c|c|c|c|}
\hline $\begin{array}{l}\text { Poster } \\
\text { Number }\end{array}$ & First Name & Last Name & Financial Relationship \\
\hline $\begin{array}{c}\text { Oral } \\
\text { Platform } \\
\text { Presentation }\end{array}$ & Ivan & da Silva & None \\
\hline $\begin{array}{c}\text { Oral } \\
\text { Platform } \\
\text { Presentation }\end{array}$ & Vahid & eslami & None \\
\hline $\begin{array}{c}\text { Oral } \\
\text { Platform } \\
\text { Presentation }\end{array}$ & Stephen & Trevick & None \\
\hline $\begin{array}{c}\text { Oral } \\
\text { Platform } \\
\text { Presentation }\end{array}$ & Michael & Reznik & None \\
\hline $\begin{array}{c}\text { Oral } \\
\text { Platform } \\
\text { Presentation }\end{array}$ & Ava & Puccio & None \\
\hline $\begin{array}{c}\text { Oral } \\
\text { Platform } \\
\text { Presentation }\end{array}$ & Santosh & Murthy & None \\
\hline $\begin{array}{c}\text { Oral } \\
\text { Platform } \\
\text { Presentation }\end{array}$ & Kyle & Hobbs & No- DASH: RO1 NS 034866-08 \\
\hline $\begin{array}{c}\text { Oral } \\
\text { Platform } \\
\text { Presentation }\end{array}$ & Stuart & Fraser & (5) \\
\hline $\begin{array}{c}\text { Oral } \\
\text { Platform } \\
\text { Presentation }\end{array}$ & Wesley & Baker & None \\
\hline $\begin{array}{c}\text { Oral } \\
\text { Platform } \\
\text { Presentation }\end{array}$ & Lynze & Franko & None \\
\hline $\begin{array}{c}\text { Oral } \\
\text { Platform } \\
\text { Presentation }\end{array}$ & Thomas & Quinn & None \\
\hline $\begin{array}{c}\text { Oral } \\
\text { Platform } \\
\text { Presentation }\end{array}$ & Stefan & Wolf & None \\
\hline $\begin{array}{c}\text { Oral } \\
\text { Platform } \\
\text { Presentation }\end{array}$ & Daniel & Hänggi & $\begin{array}{l}\text { Yes- Consultant to Edge Therapeutics } \\
\text { Inc. Manufacturer of EG-1962 }\end{array}$ \\
\hline $\begin{array}{c}\text { Oral } \\
\text { Platform } \\
\text { Presentation }\end{array}$ & Pitchaiah & Mandava & None \\
\hline 1 & Jeroen & Hermanides & None \\
\hline 2 & Yasuhiko & Ajimi & None \\
\hline 3 & Chandran & Seshagiri & Yes- Employee of Cephalogics, LLC \\
\hline 4 & Adewole & Oyalowo & None \\
\hline 5 & Neel & Singhal & None \\
\hline
\end{tabular}




\begin{tabular}{|c|c|c|c|}
\hline $\begin{array}{l}\text { Poster } \\
\text { Number }\end{array}$ & First Name & Last Name & Financial Relationship \\
\hline 6 & Sankalp & Gokhale & None \\
\hline 7 & Eric & Marrotte & None \\
\hline 8 & Polly & Pine & Yes- Portola employee \\
\hline 9 & Jonathan & Elmer & $\begin{array}{l}\text { Yes- I receive research funding from the } \\
\text { NIH/NINDS (K23-NS073806) }\end{array}$ \\
\hline 10 & Rebecca & Hammond & Yes- Employee of Sage Therapeutics \\
\hline 11 & Jennifer & Kim & None \\
\hline 12 & Prashanth & Krishnamohan & None \\
\hline 13 & Ariane & Lewis & None \\
\hline 14 & Niel & Chen & None \\
\hline 15 & Ali & Mahta & None \\
\hline 16 & Terrance & Kummer & None \\
\hline 17 & D. Ethan & Kahn & None \\
\hline 18 & Malik & Fakhar & None \\
\hline 19 & Vijay & Krishnamoorthy & None \\
\hline 20 & Sophie & Samuel & None \\
\hline 21 & Halinder & Mangat & None \\
\hline 22 & Claudia & Robertson & None \\
\hline 23 & Tomoya & Okazaki & None \\
\hline 24 & Anthony & Tannous & None \\
\hline 25 & Christopher & Melinosky & None \\
\hline 26 & HALINDER & MANGAT & None \\
\hline 27 & Brandon & Foreman, MD & None \\
\hline 28 & Andrew & Montoure & None \\
\hline 29 & Thomas & Quinn & None \\
\hline 30 & Nasim & Ahmed & None \\
\hline 31 & Brandon & Foreman, MD & None \\
\hline 32 & Casey & Rommel & None \\
\hline 33 & Sanjeev & Sivakumar & None \\
\hline 34 & Susanne & Muehlschlegel & $\begin{array}{l}\text { Yes- Susanne Muehlschlegel is supported by } \\
\text { NIH/NINDS grant K23HD080971. }\end{array}$ \\
\hline 35 & Geert & Meyfroidt & None \\
\hline 36 & Chitra & Sivasankar & None \\
\hline 37 & SHUN & SUZUKI & None \\
\hline 38 & Catalina & Sokoloff & None \\
\hline 39 & Nadia & Savy & None \\
\hline 40 & Andrew & Montoure & None \\
\hline 41 & Sebina & Bulic & None \\
\hline 42 & Molly & Hubbard & None \\
\hline 43 & Jesse & Moskowitz & None \\
\hline 44 & Hiroyuki & Nakajima & None \\
\hline 45 & Deepak & Jeswani & None \\
\hline
\end{tabular}




\begin{tabular}{|c|c|c|c|}
\hline $\begin{array}{l}\text { Poster } \\
\text { Number }\end{array}$ & First Name & Last Name & Financial Relationship \\
\hline 46 & Casey & Olm-Shipman & None \\
\hline 47 & Jeannette & Hester & None \\
\hline 48 & Yaw & Sarpong & None \\
\hline 49 & Bertan & Hallacoglu & Yes- Employee of Cephalogics, LLC. \\
\hline 50 & Brittany & Doyle & None \\
\hline 51 & Christa & Swisher & None \\
\hline 52 & Agnieszka & Ardelt & None \\
\hline 53 & Paul & McCarthy & None \\
\hline 54 & Erika & $\begin{array}{l}\text { Schlichter, BSN } \\
\text { RN }\end{array}$ & None \\
\hline 55 & Michelle & Hill & None \\
\hline 56 & Melissa & Panter & None \\
\hline 57 & Eric & Rosenthal & None \\
\hline 58 & Shannon & Appelfeller & None \\
\hline 59 & Anna & Rodriguez & $\begin{array}{l}\text { Yes- Dr. Rodriguez is an employee of Moberg } \\
\text { Research Inc. This project was supported entirely } \\
\text { by a grant from the U.S. Dept. of Defense to } \\
\text { Moberg Research. }\end{array}$ \\
\hline 60 & Lauren & Peck & None \\
\hline 61 & Mario & Kofler & None \\
\hline 62 & Chandan & Mehta & None \\
\hline 63 & Sherri & Braksick & None \\
\hline 64 & Andrew & Naidech & None \\
\hline 65 & Jeffrey & Vitt & None \\
\hline 66 & Andrew & Naidech & $\begin{array}{l}\text { Yes- This project was supported by grant number } \\
\text { K18HS023437 from the Agency for Healthcare } \\
\text { Research and Quality to Dr. Naidech. The content } \\
\text { is solely the responsibility of the authors and does } \\
\text { not necessarily represent the official views of the } \\
\text { Agency for Healthcare Research and Quality }\end{array}$ \\
\hline 67 & Peter & Abdelmalik & None \\
\hline 68 & Jamil & Dibu & None \\
\hline 69 & Nasim & Ahmed & None \\
\hline 70 & Ayham & M. Alkhachroum & None \\
\hline 71 & Joseph & Blunck & None \\
\hline 72 & Ayaz & Khawaja & None \\
\hline 73 & Kathryn & Morbitzer & None \\
\hline 74 & Neha & Dangayach & None \\
\hline 75 & Christian & Hernandez & None \\
\hline 76 & Ayaz & Khawaja & None \\
\hline 77 & Johnnie & Dillinger & None \\
\hline 78 & Truman & Milling & $\begin{array}{l}\text { Yes- Consulting for CSI Behring, PHRI. Speakers } \\
\text { Bureau for Janssen and BI. Executive Committee } \\
\text { for Portola's ANNEXA4 Study }\end{array}$ \\
\hline
\end{tabular}




\begin{tabular}{|c|c|c|c|}
\hline $\begin{array}{l}\text { Poster } \\
\text { Number }\end{array}$ & First Name & Last Name & Financial Relationship \\
\hline 79 & Andrew & Naidech & $\begin{array}{l}\text { Yes- This project was supported by grant number } \\
\text { K18HS023437 from the Agency for Healthcare } \\
\text { Research and Quality. The content is solely the } \\
\text { responsibility of the authors and does not } \\
\text { necessarily represent the official views of the } \\
\text { Agency for Healthcare Research and Quality. }\end{array}$ \\
\hline 80 & Anirudh & Sreekrishnan & None \\
\hline 81 & Audrey & Leasure & None \\
\hline 82 & Jody & Manners & None \\
\hline 83 & Christopher & Robinson & None \\
\hline 84 & Denise & Rhoney & None \\
\hline 85 & Audrey & Leasure & None \\
\hline 86 & Yahia & Lodi & None \\
\hline 87 & Christopher & Melinosky & None \\
\hline 88 & Rajat & Dhar & None \\
\hline 89 & Pitchaiah & Mandava & None \\
\hline 90 & ABDULLAH & ALAMRI & None \\
\hline 91 & Yekaterina & Axelrod & None \\
\hline 92 & Yahia & Lodi & None \\
\hline 93 & Jennifer & Frontera & None \\
\hline 94 & Lais & Medeiros & None \\
\hline 95 & Terutake & Yonemori & None \\
\hline 96 & Silvia & Schönenberger & None \\
\hline 97 & In Young & Chung & None \\
\hline 98 & Yana & Bukovskaya & None \\
\hline 99 & Sanjeev & Keshary & None \\
\hline 100 & Virginia & Roberts & None \\
\hline 101 & $\begin{array}{l}\text { ANA } \\
\text { HELENA }\end{array}$ & ANDRADE & None \\
\hline 102 & Megan & Barra & None \\
\hline 103 & Casey & Olm-Shipman & None \\
\hline 104 & Keshav & Goyal & None \\
\hline 105 & Megan & Esch & None \\
\hline 106 & Kevin & Betthauser & None \\
\hline 107 & Adam & Schertz & None \\
\hline 108 & Christina & Roels & None \\
\hline 109 & Satoshi & Egawa & None \\
\hline 110 & Naresh & Mullaguri & None \\
\hline 111 & WANG & Rong & None \\
\hline 112 & $\mathrm{Xi}$ & Liu-DeRyke & None \\
\hline 113 & Megan & Garzon & None \\
\hline 114 & Amandeep & Dolla & None \\
\hline 115 & Ariane & Lewis & None \\
\hline
\end{tabular}




\begin{tabular}{|c|c|c|c|}
\hline $\begin{array}{l}\text { Poster } \\
\text { Number }\end{array}$ & First Name & Last Name & Financial Relationship \\
\hline 116 & Abhijit & Lele & None \\
\hline 117 & Sarah & Adriance & None \\
\hline 118 & Gisele & Silva & None \\
\hline 119 & Ali & Saherwala & None \\
\hline 120 & Satoshi & Egawa & None \\
\hline 121 & Christopher & Morrison & None \\
\hline 122 & Avinash & Kumar & None \\
\hline 123 & Salvador & Cruz-Flores & None \\
\hline 124 & Karen & Lane & None \\
\hline 125 & Edilberto & Amorim & None \\
\hline 126 & Haroon & Majoka & None \\
\hline 127 & Thorsten & Steiner & Yes-Personal fees: Boehringer Ingelheim. \\
\hline 128 & Janet & Leeds & Yes- Portola employee \\
\hline 129 & Callie & Drohan & None \\
\hline 130 & Ashley & Hedges & None \\
\hline 131 & Alexandra & Baker & None \\
\hline 132 & Kelly & Gassie & None \\
\hline 133 & Anna & $\begin{array}{l}\text { Finley Caulfield, } \\
\text { MD }\end{array}$ & None \\
\hline 134 & Rachael & Schutz & None \\
\hline 135 & Genmin & Lu & Yes- Portola employee \\
\hline 136 & Fernando & Goldenberg & None \\
\hline 137 & Neha & Dangayach & None \\
\hline 138 & Fernando & Goldenberg & None \\
\hline 139 & Anil & Ramineni & None \\
\hline 140 & Mary & Barden & None \\
\hline 141 & Swarna & Rajagopalan & None \\
\hline 142 & Joseph & Haymore & None \\
\hline 143 & David & Lerner & None \\
\hline 144 & Aarti & Sarwal & None \\
\hline 145 & Charles & Francoeur & Yes- McLaughlin grant 2015-2016 \\
\hline 146 & Alexander & Merkler & None \\
\hline 147 & Anastasia & Vishnevetsky & $\begin{array}{l}\text { Yes- SUPPORTED BY NIH RESEARCH } \\
\text { TRAINING GRANT \# R25 TW009345 FUNDED BY } \\
\text { THE FOGARTY INTERNATIONAL CENTER. }\end{array}$ \\
\hline 148 & Kiruba & Dharaneeswaran & None \\
\hline 149 & Aarti & Sarwal & None \\
\hline 150 & Laith & Maali & None \\
\hline 151 & Aarti & Sarwal & None \\
\hline 152 & SungEun & Lee & None \\
\hline 153 & Michelle & Lozano & None \\
\hline 154 & Yasuhiro & Kuroda & None \\
\hline
\end{tabular}




\begin{tabular}{|c|c|c|c|}
\hline $\begin{array}{l}\text { Poster } \\
\text { Number }\end{array}$ & First Name & Last Name & Financial Relationship \\
\hline 155 & Cydni & Williams & None \\
\hline 156 & Luis & Lee & None \\
\hline 157 & Mullai & Baalaaji & None \\
\hline 158 & Binod & Balakrishnan & None \\
\hline 159 & Jennifer & Erklauer & None \\
\hline 160 & Peter & Ferrazzano & None \\
\hline 161 & Andrea & Passarelli & None \\
\hline 162 & Lynn & Do & None \\
\hline 163 & Jonathan & Rasouli & None \\
\hline 164 & Margaret & Huynh, DO & None \\
\hline 165 & Stephen & Kanes & $\begin{array}{l}\text { Yes- Employee and stockholder, Sage } \\
\text { Therapeutics, Inc. }\end{array}$ \\
\hline 166 & Theresa & Human & None \\
\hline 167 & James & Braun & None \\
\hline 168 & Helen & Colquhoun & $\begin{array}{l}\text { Yes- Employee and stockholder, Sage } \\
\text { Therapeutics, Inc. }\end{array}$ \\
\hline 169 & Jennifer & Creed & None \\
\hline 170 & John & Faro & None \\
\hline 171 & Lisa & Yanase & None \\
\hline 172 & Stephen & Kanes & $\begin{array}{l}\text { Yes- Employee and stockholder, Sage } \\
\text { Therapeutics, Inc. }\end{array}$ \\
\hline 173 & Jocelyn & Cheng & None \\
\hline 174 & Helen & Colquhoun & $\begin{array}{l}\text { Yes- Employee and stockholder Sage } \\
\text { Therapeutics, Inc. }\end{array}$ \\
\hline 175 & Michael & Mendoza & None \\
\hline 176 & Marta & Melis & None \\
\hline 177 & Raquel & Farias-Moeller & None \\
\hline 178 & Nirupama & Yechoor & None \\
\hline 179 & Chalita & Atallah & None \\
\hline 180 & Pavel & Klein & $\begin{array}{l}\text { Yes- Dr. Klein has served on advisory boards and } \\
\text { has received a research grant from Lundbeck LLC. }\end{array}$ \\
\hline 181 & Daniel & Hänggi & $\begin{array}{l}\text { Yes- Consultant to Edge Therapeutics, } \\
\text { manufacturer of EG-1962 }\end{array}$ \\
\hline 182 & Robin & Bush & None \\
\hline 183 & Sungho & Ahn & None \\
\hline 184 & Halinder & Mangat & None \\
\hline 185 & Ranjita & Raghavan & None \\
\hline 186 & Mubashir & Pervez & None \\
\hline 187 & Nancy & Edwards & None \\
\hline 188 & Jin & Park & None \\
\hline 189 & Muhammad & Athar & None \\
\hline 190 & Imad & Khan & None \\
\hline 191 & Jason & Siegel & None \\
\hline 192 & Nathaniel & Mohney & None \\
\hline
\end{tabular}




\begin{tabular}{|c|c|c|c|}
\hline $\begin{array}{l}\text { Poster } \\
\text { Number }\end{array}$ & First Name & Last Name & Financial Relationship \\
\hline 193 & Sylvia & Bele & None \\
\hline 194 & Charles & Francoeur & Yes- McLaughlin grant 2015-2016 \\
\hline 195 & David & Robinson & None \\
\hline 196 & Tamara & Majic & None \\
\hline 197 & Robert & James & $\begin{array}{l}\text { Yes- The ASTROH study is supported with funds } \\
\text { awarded to Robert F James from the University of } \\
\text { Louisville Research Foundation, investigator- } \\
\text { initiated research grants from Penumbra, Inc., and } \\
\text { MicroVention, Inc., and the } 2012 \text { Robert Simon } \\
\text { Chair of Research grant from the Brain Aneurysm } \\
\text { Foundation. }\end{array}$ \\
\hline 198 & Manoj & Mittal & None \\
\hline 199 & Siddharth & Biswal & None \\
\hline 200 & Sherry & Chou & $\begin{array}{l}\text { Yes- I receive research funding from the } \\
\text { NIH/NINDS (K23-NS073806) }\end{array}$ \\
\hline 201 & Kathryn & Morbitzer & None \\
\hline 202 & Mario & Kofler & None \\
\hline 203 & Michael & Reznik & None \\
\hline 204 & Sara & Hefton & None \\
\hline 205 & Matthew & Sharrock & None \\
\hline 206 & Eyad & Althenayan & None \\
\hline 207 & Emmaculate & Fields & None \\
\hline 208 & Tomoya & Okazaki & None \\
\hline 209 & Danyelle & Sadala & None \\
\hline 210 & Sungho & Ahn & None \\
\hline 211 & sato & hiroki & None \\
\hline 212 & Eva & Postma & None \\
\hline 213 & Apeksha & Shenoy* & None \\
\hline 214 & Kyle & Sheehan & None \\
\hline 215 & Hitoshi & Kobata & None \\
\hline 216 & Roy & Poblete & None \\
\hline 217 & Kyu Sun & Yum & None \\
\hline 218 & Hitoshi & Kobata & None \\
\hline 219 & Richard & Riker & None \\
\hline 220 & Melissa & Naiman & $\begin{array}{l}\text { Yes- Dr. Naiman is the Research Program Manager } \\
\text { and Clinical Marketing Director for Advanced } \\
\text { Cooling Therapy and receives salary and stock } \\
\text { options in the company. }\end{array}$ \\
\hline 221 & Imad & Khan & None \\
\hline 222 & Yang-Je & Cho & None \\
\hline 223 & Stephen & Griffiths & None \\
\hline 224 & Zakraus & Mahdavi & None \\
\hline 225 & Vijayalekshmi & Nair & None \\
\hline 226 & Elizabeth & Carroll & None \\
\hline
\end{tabular}




\begin{tabular}{|c|c|c|c|}
\hline $\begin{array}{l}\text { Poster } \\
\text { Number }\end{array}$ & First Name & Last Name & Financial Relationship \\
\hline 227 & Asher & Albertson & None \\
\hline 228 & Chun Fai & Cheah & None \\
\hline 229 & Huy & Tran & None \\
\hline 230 & Manoj & Mittal & $\begin{array}{l}\text { Yes- I am the medical director for Ornim who } \\
\text { makes cFLOW. }\end{array}$ \\
\hline 231 & March & Rucci & None \\
\hline 232 & Sowmya & Kumble & None \\
\hline 233 & Anand & Venkatraman & None \\
\hline 234 & Elena & Schmidt & None \\
\hline 235 & Julian & Macedo & None \\
\hline 236 & Kinjal & Desai & None \\
\hline 237 & Nikhil & Madan & None \\
\hline 238 & Kelly & Braun & None \\
\hline 239 & Syeda & Alqadri & None \\
\hline 240 & Bibhukalyani & Das & None \\
\hline 241 & Megan & Lange & None \\
\hline 242 & Eileen & Vallin & None \\
\hline 243 & Stasia & Rouse & None \\
\hline 244 & Soo & Jeong & None \\
\hline 245 & Lara & Zimmermann & None \\
\hline 246 & Zaid & Aljuboori & None \\
\hline 247 & Biplab & Saha & None \\
\hline 248 & Firas & $\begin{array}{l}\text { Abdulmajeed, } \\
\text { MB. ChB }\end{array}$ & None \\
\hline 249 & Sung & Cho & None \\
\hline 250 & Ayush & Batra & None \\
\hline 251 & Amandeep & Dolla & None \\
\hline 252 & Jonathan & Marehbian & None \\
\hline 253 & $\begin{array}{l}\text { Anantha } \\
\text { Ramana }\end{array}$ & Vellipuram & None \\
\hline 254 & Michelle & Nunes & None \\
\hline 255 & Michelle & Nunes & None \\
\hline 256 & Bibhukalyani & Das & None \\
\hline 257 & Lauren & Koffman & None \\
\hline 258 & Avni & Kapadia & None \\
\hline 259 & Simona & Ferioli, MD & None \\
\hline 260 & Flavio & Carvalho & None \\
\hline 261 & Christoph & Stretz & None \\
\hline 262 & Tapan & Kavi & None \\
\hline 263 & Jesse & Robertson & None \\
\hline 264 & Angela & Vizzini & None \\
\hline 265 & Marin & Darsie & None \\
\hline 266 & Adam & Lewis & None \\
\hline
\end{tabular}

